\author{
Universidade de São Paulo \\ Instituto de Física
}

\title{
Efeitos de um Vácuo Dinâmico na Evolução Cósmica e no Colapso Gravitacional
}

\author{
Eder Leonardo Duarte Perico
}

Orientador: Prof. Dr. José Ademir Sales de Lima

Tese de doutorado apresentada ao Instituto de Física para a obtenção do título de Doutor em Ciências

Banca Examinadora:

Prof. Dr. José Ademir Sales de Lima (IAG/USP)

Prof. Dr. Carlos Molina Mendes (EACH/USP)

Prof. Dr. Ioav Waga (UFRJ)

Prof. Dr. George Emanuel Avraam Matsas (IFT/UNESP)

Prof. Dr. Rudnei de Oliveira Ramos (UERJ) 


\section{FICHA CATALOGRÁFICA}

\section{Preparada pelo Serviço de Biblioteca e Informação}

do Instituto de Física da Universidade de São Paulo

\section{Duarte Perico, Eder Leonardo}

Efeitos de um vácuo dinâmico na evolução cósmica e no colapso gravitacional. São Paulo, 2015.

Tese (Doutorado) - Universidade de São Paulo. Instituto de Física.

Orientador: Prof. Dr. José Ademir Sales de Lima

Área de Concentração: Cosmologia

Unitermos: 1. Cosmologia; 2. Inflação; 3. Buracos negros. 


\section{A GRADECIMENTOS}

Dedico este trabalho à minha mãe e ao meu pai, lhes agradeço imensamente pelo apoio e carinho incondicional que sempre tenho recebido de suas partes, pois sem eles não seria quem sou no presente. Devo a eles a vida toda. Agradeço também aos meus irmãos, sobrinhos e tios, pela paciência e carinho.

Agradeço ao Professor José Ademir Sales de lima, pela sugestão do tema, pela sua orientação ao longo de todo o doutorado, pela sua colaboração na obtenção de cada um dos resultados apresentados neste trabalho, e não menos importante, por seus conselhos.

Agradeço ao Professor e amigo Miguel de Campos, por me haver iniciado na física do colapso gravitacional e por sua colaboração nos trabalhos referentes ao tema; ao George Ziliti por ter trabalhado junto na análise do modelo deflacionário com curvatura espacial; como também aos Professores Joan Solà e Spyros Basilakos, por sua colaboração no desenvolvimento do trabalho referente ao cenário cosmológico completo. Agradeço também aos professores aos Professores Marcos Lima e Carlos Molina, e a todos os professores do IF que contribuíram para minha formação acadêmica.

Agradeço aos meus colegas de trabalho no Instituto de Física e no IAG, em particular à Leila, Alejandro e Hugo; e ao todos meus amigos do Instituto de Física.

Agradeço ao CNPq pelo apoio financeiro durante os 4 anos do meu doutorado no IF. 



\section{RES UMO}

As observações astronômicas dos últimos 15 anos revelaram que o universo atualmente está expandindo aceleradamente. No contexto da relatividade geral se acredita que a energia escura, cujo melhor candidato é a densidade do vácuo $(\Lambda / 8 \pi G)$, é o agente responsável por este estado acelerado. No entanto, o termo $\Lambda$ tem duas sérias dificuldades: o problema da constante cosmológica e o problema da coincidência.

Com o objetivo de aliviar o problema da constante cosmológica, muitos modelos adotam um termo $\Lambda$ dinâmico, permitindo seu decrescimento ao longo de toda a história cósmica. Neste tipo de modelo, a equação de conservação do tensor momento energia total exige uma troca de energia entre a densidade do vácuo e as outras componentes energéticas do universo; o que também alivia o problema da coincidência.

Neste trabalho discutimos diferentes consequências de um vácuo dinâmico no âmbito cosmológico e no processo de colapso gravitacional. Em particular, analisamos o caso em que a densidade do vácuo possui uma dinâmica não trivial com a escala de energia típica do universo, que depende monotonamente do parâmetro de Hubble, decrescendo ao longo de toda a história cósmica. Nos referiremos a este modelo como modelo deflacionário.

Nesse contexto, utilizando os primeiros termos da expansão para a densidade do vácuo, sugerida pela teoria do grupo de renormalização em espaço-tempos curvos, propomos um novo cenário cosmológico baseado numa densidade do vácuo dinâmica. O cenário proposto é completo no sentido de que o mesmo vácuo é responsável pelas duas fases aceleradas do universo, conectadas por uma fase de radiação e um estágio de domínio da matéria.

Neste cenário o universo plano é não singular, iniciando sua evolução a partir de um estágio do tipo de Sitter e, portanto, toda a história cósmica ocorre entre duas fases de Sitter limites. Este modelo não apresenta o problema de horizonte, e nele a nucleossíntese cosmológica ocorre como no modelo de Friedmann, e embora este modelo seja muito próximo do modelo $\Lambda \mathrm{CDM}$, o grande acúmulo de observações no estágio recente do universo permitirão que este poda ser testado. Adicionalmente, mostraremos que generalizações do modelo deflacionário incluindo curvatura espacial apresentam propriedades e vantagens similares.

Usando observações de $H(z)$, da luminosidade de supernovas tipo Ia, da função de crescimento linear das perturbações escalares, e da posição do pico das oscilações acústicas de bárions conseguimos vincular um dos parâmetros do modelo. Por outro lado, analisando a física do universo primordial, assumindo um vácuo não perturbado, conseguimos limitar um segundo parâmetro fazendo uso do índice espectral das perturbações escalares.

Com o objetivo de fazer uma análise mais completa do modelo no âmbito cosmológico, analisamos também as possíveis restrições oriundas da validade da segunda lei da termodinâmica em sua forma generalizada (GSLT). Para isto investigamos a evolução tanto da entropia associada ao horizonte apa- 
rente do universo, que é um horizonte atrapante devido a que o escalar de Ricci é positivo, como do seu conteúdo material.

Motivados pela forma como a singularidade primordial do universo é evitada devido aos efeitos do decaimento do vácuo, incluímos no presente trabalho outra linha de desenvolvimento: a análise dos estágios finais do processo de colapso gravitacional em presença de uma densidade do vácuo dinâmica. Centraremos esta análise na determinação de modelos que possam ou não evitar a formação de um buraco negro.

Mostraremos que modelos com um termo de vácuo proporcional à densidade de energia total do sistema, não podem evitar a formação de uma singularidade no estágio final do processo de colapso. Adicionalmente obteremos correções para a massa colapsada, para o tempo de formação do horizonte e para o tempo de colapso como função dos parâmetros do modelo e da curvatura espacial.

Por último analisaremos a influência de uma densidade do vácuo capaz de dominar sobre as outras componentes no regime de altas energias, mostrando que este tipo de dinâmica na densidade do vácuo evita a formação de um estado final singular.

Palavras Chaves: Problema da constante cosmológica; Problema da coincidência; Cosmologias com vácuo dinâmico; Inflação; Colapso gravitacional.

Áreas do conhecimento: Cosmologia; Gravitação. 


\section{ABSTRACT}

The astronomical observations of the last 15 years revealed that the universe is currently undergoing an expanding accelerating phase. In the general relativity context is believed that dark energy, whose best candidate is the vacuum energy density $\rho_{v} \equiv \Lambda / 8 \pi G$, is the fuel responsible for the present accelerating stage. However, the so-called $\Lambda$-term has two serious drawnbacks, namely: the cosmological constant problem and the coincidence problem.

In order to alleviate the cosmological constant problem, many models adopt a dynamical $\Lambda$ term, thereby allowing its decreasing throughout the cosmic history. In this kind of model, the total energy conservation law defined in terms of the energy momentum tensor requires an energy exchange between the vacuum and the material components of the universe, which also contributes to alleviate the coincidence problem.

In the present thesis we discuss different consequences of an interacting vacuum component both in the cosmological scenario as well as in the process of gravitational collapse. In particular, in the cosmological domain, we examine the case where the vacuum has a nontrivial dynamics dependent on a typical energy scale, the Hubble parameter, that decreases in the course of the cosmic history. We will refer to this model as deflationary model.

In this context, by using a truncated expansion for the vacuum energy density, as suggested by the renormalization group theory in curved space-time, we propose a new cosmological scenario based on a dynamical $\Lambda$-term. The proposed scenario is complete in the sense that the same vacuum is responsible for both accelerating phases of the universe, which are linked by two subsequent periods of radiation and non-relativistic matter domination.

In this scenario the flat universe is nonsingular and starts its evolution from an asymptotic de Sitter stage, so that the cosmic story takes place between two extreme de Sitter phases. The model is free of the horizon problem as well as of the "graceful exit"problem plaguing many inflationary variants. In addition, the cosmological nucleosynthesis occurs as in the Friedmann model and the observations in the latest stages of the universe can potentially differentiate between the deflationary and the standard $\Lambda \mathrm{CDM}$ model. The generalizations including spatial curvature are aslo discussed in detail.

On the other hand, by using the late time tests like type Ia supernovae, the redshift dependence of the Hubble parameter, $H(z)$, the linear growth function of scalar perturbations, and the peak position of baryon acoustic oscillations we have constrained the basic parameters of the model. Conversely, analyzing the physics of the primordial universe and assuming that the vacuum is a smooth component, we have also constrained the spectral index of scalar density perturbations.

In order to establish a more complete analysis of our cosmological scenario, we also discuss the possible constraints arising from the validity of the generalized second law of thermodynamics, that is, by including the horizon thermodynamics. Since the apparent horizon of the universe behaves like a 
trapped horizon because the Ricci scalar is positive, we investigate the evolution of both the entropy of the material components and the entropy associated to the horizon.

Motivated by the avoidance of the Big-Bang singularity due to the decaying vacuum effects, we have explored another line of development: the analysis of the final stages of gravitational collapse process in the presence of a dynamic vacuum. This analysis focused on the determination of models able to prevent or not the formation of a black hole.

In this connection, we shown that the presence of an interacting vacuum proportional to the total energy density of the system does not prevent the formation of a singularity in the final stages of the collapsing process. In addition, we obtain corrections for the collapsed mass, the horizon time formation and the collapsing time as a function of the free parameters and the spatial curvature of the models.

Finally, we have also analyzed the influence of a vacuum contribution which dominates the other components into the high energy limit (due to the presence of higher orders terms in the contraction rate), and shown that for this kind of models the growth of the vacuum energy density prevents the formation of the singularity.

Key Words: Cosmological constant problem; Coincidence problem; Cosmologies with a dynamical vacuum; Inflation; Gravitational collapse.

Areas of knowledge: Cosmology; Gravitation. 


\section{NOMENCLATURA}

$\tilde{a} \quad$ Fator de escala no começo da inflação, 71

$a_{0} \quad$ Fator de escala hoje para um universo em expansão, 16

$a_{\star} \quad$ Fator de escala no fim da inflação, $q\left(a_{\star}=0\right), 65$

a $\quad$ Fator de escala, veja equação (2.12), 10

$a_{e q} \quad$ Fator de escala quando as densidades de energia da radiação de da matéria foram iguais, 19

$\mathscr{A}_{h} \quad$ Área do horizonte aparente do universo, veja equação (7.3), 89

$a_{l s} \quad$ Fator de escala na última superfície de espalhamento dos fótons, 41

$a_{l t} \quad$ Fator de escala quando a aceleração é nula no universo recente, 78

$\varrho_{i} \quad$ Constante de integração, 7

$C_{i} \quad$ Constante de integração, 7

$c_{i} \quad$ Constante de integração, 7

$c_{s}^{2} \quad$ Cociente das perturbações na pressão e na densidade para um fluido, 34

$\chi \quad$, veja equação (6.10), 65

$\Delta \quad$, veja equação (9.6), 102

$\delta \quad$ Contraste de densidade, veja equação (3.29), 30

1 Derivada em relação ao fator de escala, 71

, Derivada parcial, 8

; $\quad$ Derivada covariante, veja equação (2.2), 8

· $\quad$ Derivada em relação ao tempo conforme, 11

- Derivada em relação ao tempo, 120

$\epsilon \quad$ Parâmetro de rolamento lento para a inflação, veja equação (4.13a), 44

E Parâmetro de Hubble normalizado no período inflacionário $H / H_{I}$, veja equação $(8.21), 99$

$\eta_{c} \quad$ Tempo conforme de colapso, no processo de colapso gravitacional, 106

$\eta \quad$ Tempo conforme, veja equação (2.15), 11

$g^{\mu \nu} \quad$ Tensor métrico, veja equação (2.1), 7 
$\gamma \quad$ Parâmetro da equação de estado de um gás ideal, veja equação (8.2), 95

$\Gamma_{\alpha \beta}^{\mu} \quad$ Símbolo de Christofel, veja equação $(2.2), 8$

gut Escala de energia da grande unificação, 44

$\mathcal{H} \quad$ Parâmetro de Hubble comóvel, veja equação (2.15), 11

$\mathcal{H}^{-1} \quad$ Raio de Hubble comóvel, 12

H Taxa de contração de um sistema que colapsa gravitacionalmente, 102

H Parâmetro de Hubble, veja equação (2.15), 11

h Horizonte aparente, 142

$H^{-1} \quad$ Raio de Hubble, 12

$H_{I} \quad$ Parâmetro de Hubble no começo da inflação, veja equação (6.7), 64

h Perturbações tensoriais da métrica, veja equação (3.74), 38

k Vetor de onda no espaço de Fourier, 33

$K_{a b} \quad$ Segunda forma fundamental de uma hiper-superfície, veja equação (A.14), 131

$\kappa \quad$ Curvatura espacial normalizada, veja equação (2.17), 11

$\lambda \quad$ Segundo parâmetro de rolamento lento para a inflação, veja equação (4.13), 45

$\ell_{p l} \quad$ Comprimento de Planck, 89

m $\quad$ Função massa para um sistema esfericamente simétrico, veja equação (B.7), 142

$M_{p l} \quad$ Escala de energia de Planck, 61

$M_{\odot} \quad$ Massa do sol, 109

$M_{X} \quad$ Escala de energia onde o modelo padrão da física de partículas perde validade, 61

$n_{\phi} \quad$ Índice espectral da perturbação escalar $\phi$, veja equação (6.33), 73

$n_{s} \quad$ Índice espectral escalar, veja equação (4.43), 50

$\omega \quad$ Parâmetro da equação de estado para um fluido, $\mathcal{P} \equiv \omega \rho, 15$

$\Omega_{i} \quad$ Fração da densidade de energia do fluido $i$ em relação à densidade crítica, veja equação (2.38), 16

$\mathcal{P} \quad$ Pressão de um fluido, veja equação $(2.29), 14$

$\varphi \quad$ Campo escalar inflacionário, 45

$P \quad$ Espectro de potências, veja equação (3.60), 36

$q$ Parâmetro de desaceleração, veja equação (6.5), 64 
$\mathscr{R} \quad$ Raio físico, $\mathscr{R}=a r$, onde $r$ é o raio comóvel e $a$ é o fator de escala, 89

$R \quad$ Raio físico de um núcleo estelar em processo de colapso gravitacional, veja equação (9.24), 107

$R_{\circledast} \quad$ Raio do núcleo estelar no começo da era de Sitter final do colapso, 118

$r_{\Sigma} \quad$ Raio comóvel de um núcleo estelar em processo de colapso gravitacional, veja equação (9.24), 107

$R_{S \odot} \quad$ Raio de Schwartzschild associado à massa do sol, 109

$R_{c} \quad$ Raio de um núcleo estelar quando a aceleração é nula nos estágios avançados do colapso gravitacional, veja equação (10.18), 119

$\mathcal{R} \quad$ Escalar de Ricci, veja equação (2.10), 9

$R_{\beta \mu v}^{\alpha} \quad$ Tensor de Riemann, veja equação (2.8), 9

$R_{\mu v} \quad$ Tensor de Ricci , veja equação (2.9), 9

$\rho \quad$ Densidade de energia de um fluido, veja equação (2.29), 14

$\rho_{c r} \quad$ Densidade crítica do universo, 16

$\mathcal{S} \quad$ Entropia de um volumem comóvel fixo, 89

$\sigma \quad$ Perturbação anisotrópica do tensor momento energia, veja equação (3.45), 33

$\Sigma^{i j} \quad$ Perturbação espacial anisotrópica do tensor momento energia, veja equação (3.29), 30

T Temperatura, 38

t $\quad$ Tempo físico, veja equação $(2.11), 10$

$t_{c} \quad$ Tempo de colapso, no processo de colapso gravitacional, 104

$\theta \quad$ Perturbação espaço-temporal do tensor momento energia, veja equação (3.43), 33

$T^{\mu \nu} \quad$ Tensor momento energia, veja equação (2.28), 13

$V \quad$ Potencial associado ao campo escalar inflacionário, veja equação (4.14), 45

$v \quad$ Velocidade peculiar, 30

$\xi \quad$, veja equação $(6.4), 64$

$\zeta \quad$ Perturbação de iso-curvatura invariante de calibre, veja equação (3.33), 31 



\section{CONTEÚDO}

Capa i

Agradecimentos iii

Resumo v

Abstract vii

Notação xi

Conteúdo $\quad \mathrm{xv}$

Lista de figuras xviii

1 INTRODUÇÃO 1

2 COSMOLOGIAS HOMOGÊNEAS E ISOTRÓpicAs 7

2.1 Geometria do universo 7

2.1.1 Expansão do universo 10

2.2 Dinâmica do universo em grandes escalas 13

3 PerturbaÇões lineares no universo frW plano 23

3.1 Perturbações lineares no tensor métrico 24
3.1.1 Liberdade de calibre
25

3.2 Perturbações escalares 28

3.2.1 Perturbações no tensor momento energia 30

3.2.2 Invariança de calibre 30

3.2.3 Conservação do tensor momento energia 33

3.2.4 Função de correlação e espectro de potências 35

3.3 Perturbações tensoriais 37

3.3.1 Ondas gravitacionais 37

4 INFLAÇ ÃO 41

4.1 Parâmetros de rolamento lento $\quad 44$

4.2 Implementação da inflação usando um campo escalar 45

4.2.1 Perturbações do campo escalar inflacionário $\quad 46$

4.2.2 Valor esperado das perturbações e espectro primordial 48

4.3 Evolução das perturbações tensoriais durante a inflação 50

4.4 Necessidade de reaquecimento após a inflação $\quad 52$

5 DENSIDAde do VÁCUO VARIÁVEL 53

5.1 Universo em expansão com uma densidade do vácuo que decresce monotonamente 53

5.1.1 Universos com eras de Sitter inicial e final $\quad 54$

5.1.2 Universos em que $3 H^{2}$ cresce mais rápido do que a densidade do vácuo $\Lambda \quad 57$

5.1 .3 Resumo 58

5.2 Proposta fenomenológica para a forma de $\Lambda(t) \quad 58$

5.3 Vácuo dinâmico e grupo de renormalização 60 
6 Universo EM EXPANSÃo COM UMA DENSIDADE DO VÁCUO DINÂMICA

6.1 Universo primordial 64

6.1.1 Inflação e período de domínio da radiação $\quad 66$

6.2 Potencial efetivo equivalente à densidade total no universo primordial 69

6.3 Perturbações escalares no universo primordial $\quad 70$

6.3.1 Espectro de potências primordial 71

6.3.2 Escala pivô $\quad 74$

6.4 Universo recente 75

6.4.1 Domínio da matéria e do vácuo remanescente $\quad 76$

6.4.2 Aliviando os problemas da coincidência e da constante cosmológica

6.5 Ajustando o parâmetro $v \quad 80$

6.5.1 Parâmetro de Hubble 82

6.5.2 Função de crescimento das perturbações lineares $\quad 82$

6.6 Resultados 84

6.7 Resumo do capítulo 84

7 Modelo deflacionário e segunda lei da termodinâmica 89

7.1 Entropia associada ao horizonte cosmológico aparente $\quad 89$

7.1.1 Universo primitivo 90

7.1.2 Universo recente 90

7.2 Temperatura de um fluido ideal 91

7.3 Entropia dos fluidos contidos no horizonte 92

7.3.1 Instabilidade do período inflacionário $\quad 92$

7.3.2 Estabilidade da era de Sitter final do universo $\quad 93$

8 universo deflacionário e curvatura espacial 95

8.1 O modelo 95

8.2 Casos limite 97

8.3 Dinâmica do modelo 98

8.4 Intervalo de validade do modelo 99

9 Colapso gravitacional Com um VÁCuo dinâmico e Curvatura espacial 101

9.1 Dinâmica do sistema 101

9.2 Formação do horizonte aparente 107

9.3 Condições de Junção 110

9.4 Resumo do capítulo 112

10 Colapso gravitacional Com VÁCuo dinÂmico 113

10.1 Geometria e composição do sistema 113

10.1.1 Estágio intermediário do processo de colapso 117

10.1.2 Estágio de Sitter final 118

10.2 Estágios acelerados e desacelerados 119

10.3 Horizonte aparente 120

10.3.1 Quantos horizontes aparentes podem se formar 120

10.3.2 Qual é o raio dos horizontes para $\mathrm{H}_{i} \ll \mathrm{H}_{f} \quad 123$

10.3.3 Massa do sistema 124

10.4 Resumo do capítulo 125 
11 CONSIDERAÇÕES FINAIS 127

A alguns elementos Úteis de geometria e buracos negros

A.1 Hiper-superfícies 129

A.2 Estrutura causal do espaço-tempo 131

A.2.1 Superfícies de Cauchy 132

A.2.2 Estrutura conforme da fronteira do espaço-tempo de Minkowsky

A.2.3 Outros Horizontes 133

A.2.4 Completeza geodésica 134

A.2.5 Geodésicas temporais 134

A.2.6 Geodésicas nulas 135

A.2.7 Buracos negros em espaços assintoticamente simples 136

B ESPAÇO-TEMPos COM Simetria esférica E BURACOS NEgRos

B.1 Horizonte aparente 142

B.2 Condições de junção 143

B.3 Métrica interna 143

B.3.1 Equações de Einstein 144

B.4 Métrica externa 146

Referências 153 



\section{LISTA DE FIGURAS}

Figura 2.1

Figura 2.2

Figura 2.3

Figura 2.4

Figura 4.1

Figura 4.2

Figura 4.3

Figura 4.4

Figura 5.1

Figura 5.2

Figura 5.3

Figura 5.4

Figura 6.1

Figura 6.2

Figura 6.3

Figura 6.4

Figura 6.5

Figura 6.6

Figura 6.7

Figura 6.8

Figura 6.9

Figura 6.10

Figura 6.11

Figura 6.12

Figura 6.13

Figura 6.14

Figura 8.1

Laço infinitesimal definido pelos vetores $A^{\mu}$ e $B^{\mu}$.

9

Expansão homogênea e isotrópica do universo. 10

Evolução da densidade de energia da radiação, da matéria escura fria e da energia escura

17

Evolução do fator de escala como função do tempo conforme para o modelo $\Lambda C D M$.

20

Solução da inflação ao problema de horizonte.

43

Solução da inflação ao problema de horizonte.

43

Evolução das perturbações escalares da métrica durante uma inflação exponencial. $\quad 46$

Evolução das perturbações tensoriais e escalares durante uma inflação exponencial. $\quad 50$

Universos com eras aceleradas inicial e final, e um estágio intermediário desacelerado.

55

Universos acelerados exceto em um instante de tempo intermediário.

56

Universos que se expandem sempre aceleradamente. $\quad 56$

Universos com uma era inicial desacelerada e uma era final acelerada. $\quad 57$

Evolução das densidades no universo primordial com $\Lambda=3 v H^{2}+3(1-v) H^{2+n} / H_{I}^{n}$.

Evolução das densidades no universo primordial com $\Lambda=3 v H^{2}+3(1-v) H^{2+n} / H_{I}^{n}$.

Parâmetro de desaceleração durante as eras primordiais com $\Lambda=3 v H^{2}+3(1-$ v) $H^{2+n} / H_{I}^{n}$.

69

Termos que dominam a evolução das perturbações escalares durante o período deflacionário.

70

Como as escalas saem e entram no horizonte por causa da inflação.

70

Índice espectral das perturbações escalares no modelo deflacionário.

72

Evolução das perturbações escalares durante a deflação.

74

Parâmetro de desaceleração na era recente para o modelo $\Lambda=\Lambda_{c}+3 v H^{2}$.

78

Evolução das densidade no universo recente para o modelo com $\Lambda=\Lambda_{c}+$ $3 v H^{2}$.

79

Quebra da degenerescência do sistema no espaço dos parâmetros $v$ e $\Omega_{m}$.

80

Distribuição dos parâmetros do modelo obtida das análises separadas e conjunta.

85

Melhor ajuste do modelo deflacionário e do modelo padrão.

86

Distribuição de probabilidade dos parâmetros ajustados usando a análise conjunta

87

Parâmetro de desaceleração no modelo completo com $\Lambda=\Lambda_{c}+H^{2}\left(3 v+\left(H / H_{I}\right)^{n}\right)$.

Dinâmica do modelo deflacionário com curvatura espacial.

99

xvii 
Figura 8.2 Evolução da densidade de energia da radiação durante a deflação com curvatura. 100

Figura 9.1 Densidade de energia material durante o colapso gravitacional no modelo $\Lambda=$ $c_{0}+3 v\left(\mathrm{H}^{2}+\tilde{\kappa} / R^{2}\right) . \quad 103$

Figura 9.2 Evolução do raio de um sistema que colapsa no modelo com $\Lambda=c_{0}+3 v\left(\mathrm{H}^{2}+\right.$ $\left.\kappa / a^{2}\right) . \quad 105$

Figura 9.3 Tempo de formação do horizonte no processo de colapso com $\Lambda=c_{0}+3 v\left(\mathrm{H}^{2}+\right.$ $\left.\tilde{\kappa} / R^{2}\right) . \quad 108$

Figura 9.4 Massa do buraco negro formado no colapso gravitacional com $\Lambda=c_{0}+3 v\left(\mathrm{H}^{2}+\right.$ $\left.\tilde{\kappa} / R^{2}\right) . \quad 110$

Figura 10.1 Raio do núcleo estelar durante os estágios avançados do colapso com $\Lambda=c_{0}+$ $3 v \mathrm{H}^{2}+\gamma \mathrm{H}^{4} . \quad 114$

Figura 10.2 Evolução das densidades nos estágios avançados do colapso com $\Lambda=c_{0}+3 v \mathrm{H}^{2}+$ $\gamma \mathrm{H}^{4} . \quad 115$

Figura 10.3 Formação dos horizontes aparentes no colapso gravitacional com $\Lambda=c_{0}+$ $3 v \mathrm{H}^{2}+\gamma \mathrm{H}^{4} . \quad 118$

Figura 10.4 Evolução da função $\stackrel{\circ}{R}^{2}$ durante o processo de colapso com $\Lambda=3 v \mathrm{H}^{2}+\gamma \mathrm{H}^{4} . \quad 121$

Figura 10.5 Espaço de parâmetros com 2, 1 ou 0 horizontes no colapso com $\Lambda=3 v \mathrm{H}^{2}+$ $\gamma \mathrm{H}^{4} . \quad 123$ 


\section{N T R O D U Ç Ã O}

Os dados observacionais provenientes de supernovas tipo 1a, anisotropias na radiação cósmica de fundo, aglomerados de galáxias e oscilações acústicas de bárions, indicam fortemente um estágio acelerado para a presente fase de expansão do Universo [Spergel et al., 2003, 2007; Copeland et al., 2006; Frieman et al., 2008; Kowalski et al., 2008; Hicken et al., 2009; Hinshaw et al., 2009; Komatsu et al., 2009, 2011; Planck Collaboration, 2014]. Esta expansão acelerada é atribuída a uma componente energética com pressão negativa, chamada de energia escura, e que deve dominar o universo recentemente. O modelo mais simples para energia escura não interagente, chamado de constante cosmológica $\Lambda$ [Padmanabhan, 2003; Peebles and Ratra, 2003], a descreve como sendo um fluido ideal com equação de estado $\omega_{\Lambda} \equiv$ $\mathcal{P}_{\Lambda} / \rho_{\Lambda}=-1$, e em conjunto com a matéria escura fria $(\mathrm{CDM})$ e a radiação (como sendo os fluidos que dominam a evolução do universo) compõem o modelo cosmológico padrão $(\Lambda \mathrm{CDM})$.

Inclusive antes das evidências da aceleração do universo, obtidas a partir da observação de supernovas distantes [Riess et al., 1998; Perlmutter et al., 1998, 1999], a constante cosmológica foi introduzida [Krauss, 1998] para resolver o problema da idade do universo nos modelos cosmológicos dominados por radiação e matéria escura fria. Embora o sucesso fenomenológico do modelo $\Lambda$ CDM, que concorda com as observações astronômicas mais recentes (incluindo tanto a evolução de fundo quanto o nível perturbativo), a constante cosmológica apresenta problemas teóricos sérios tais como o problema de ajuste fino conhecido como o problema da constante cosmológica [Weinberg, 1989], e o problema da coincidência.

O primeiro surge ao considerar a energia do vácuo quântico $\left\langle\rho_{v}\right\rangle$ das diferentes componentes materiais do universo (que obedece a equação de estado $\left\langle\mathcal{P}_{v}\right\rangle=-\left\langle\rho_{v}\right\rangle$ ) como uma densidade de energia gravitante. Se dita densidade do vácuo $\left\langle\rho_{v}\right\rangle$ for não nula, a soma dela com uma constante cosmológica $\rho_{c}$ deveriam gerar a energia escura efetiva observada hoje $\rho_{\Lambda}=\left\langle\rho_{v}\right\rangle+\rho_{c}$. Para lograr isto devemos ter que $\rho_{c}=$ $-\left(1-10^{-118}\right) \times 10^{71} \mathrm{GeV}^{4}$, introduzindo um ajuste fino de 118 casas decimais, já que a densidade da energia escura observada hoje é da ordem de $\rho_{\Lambda} \sim 10^{-47} \mathrm{GeV}^{4}$ (obtida a partir das observações cosmológicas), enquanto que o valor esperado do vácuo é da ordem de $\left\langle\rho_{v}\right\rangle \sim 10^{71} \mathrm{GeV}^{4}$ [Weinberg, 1989; Zel'dovich, 1968] ( estimado pela teoria quântica de campos assumindo a validade do modelo padrão da física de partículas até a escala da energia de Planck).

O segundo problema está relacionado com a impossibilidade de explicar como uma densidade de energia escura constante $\left(r h o_{\Lambda}\right.$ no contesto $\left.\Lambda \mathrm{CDM}\right)$ pode ter justamente a mesma ordem de magnitude que a densidade de energia da matéria escura hoje, já que esta última decresce de forma proporcional a $a^{-3}$ ao longo de toda a historia cósmica do universo. 
Por outro lado, no modelo padrão da cosmologia (inflação $+\Lambda \mathrm{CDM}$ ), o universo emerge de uma singularidade evoluindo ao longo de 4 estágios bem diferenciados: inflação, domínio da radiação, domínio da matéria escura e o período de aceleração recente (dominado pela energia escura). Durante as eras de domínio da radiação e da matéria escura a expansão do universo acontece de forma desacelerada, enquanto que durante os períodos recente e inflacionário a expansão é acelerada. Atualmente desconhecemos qualquer conexão entre os mecanismos responsáveis pelos dois períodos de expansão acelerada.

Uma forma natural de atenuar esta série de problemas consiste em assumir que o valor da constante cosmológica é identicamente nulo, $\rho_{c} \equiv 0$. Neste caso, a densidade da energia escura passa a ser igual ao valor esperado do vácuo, $\rho_{\Lambda}=\left\langle\rho_{v}\right\rangle$, e relaxar a suposição de que esta possui um valor estático, passando de $\left\langle\rho_{v}\right\rangle=$ cte para $\left\langle\rho_{v}\right\rangle(t)$, mas mantendo inalterada sua equação de estado. Este tipo de dinâmica foi proposta inclusive antes das observações que levaram a concluir que hoje o universo se expande aceleradamente [Freese et al., 1987; Ozer and Taha, 1987; Ratra and Peebles, 1988; Chen and Wu, 1990; Pavón, 1991; Carvalho et al., 1992; Salim and Waga, 1993; Arbab and Abdel-Rahman, 1994; Wetterich, 1995; Bloomfield Torres and Waga, 1996; Arbab, 1997; Ferreira and Joyce, 1998; Overduin and Cooperstock, 1998]. Neste contesto, modelos não singulares iniciando a evolução em um estágio de Sitter primordial foram propostos por Lima e colaboradores [Lima and Maia, 1994; Lima and Trodden, 1996]. Após as observações de supernovas que revelaram um universo recentemente dominado por energia escura, aumentou o interesse nos modelos com uma densidade do vácuo que decresce ao longo da historia térmica. Assim, o vácuo primordial poderia assumir uma densidade igual ao valor esperado pela teoria quântica de campos $\sim 10^{71} \mathrm{GeV}^{4}$ e decrescer ao longo de toda a história cósmica até atingir o valor observado atualmente $\sim 10^{-47} \mathrm{GeV}$. Uma família de modelos de energia escura ainda mais interessante descreve o universo com eras de Sitter inicial e final [Carneiro, 2006; Carneiro and Tavakol, 2009; Lima et al., 2013] reduzindo o setor escuro + inflacionário ao setor escuro, e é este tipo de modelo que estudamos neste trabalho.

A variação no valor esperado do vácuo (como sendo a função completa de Green sem propagadores externos associados às interações forte e eletro-fraca), motivada desde o ponto de vista do grupo de renormalização, tem sido estudada no cenário de espaços tempos curvos [Adler, 1982; Nelson and Panangaden, 1982; Toms, 1983; Parker and Toms, 1985; Buchbinder et al., 1992; Babić et al., 2002; España-Bonet et al., 2004; Babić et al., 2005; Shapiro et al., 2005; Grande et al., 2011], como também incluindo ao mesmo tempo uma variação no parâmetro de acoplamento gravitacional G [Solà, 2008; Grande et al., 2011]. A pesar de não providenciar a forma funcional exata para a variação do valor esperado do vácuo, a maioria desses trabalhos motivam sua parametrização como função da densidade total do universo, escrita muitas vezes como uma expansão em séries de potencias pares do parâmetro de Hubble. Este tipo de modelos já foram confrontadas com dados provenientes de supernovas, oscilações acústicas de bárions, formação de estruturas, e anisotropias na radiação cósmica de fundo, que são todos consistentes com este classe de dinâmica não trivial para a densidade da energia escura [Carvalho et al., 1992; Arcuri and Waga, 1994; Shapiro et al., 2003; España-Bonet et al., 2004; Opher and Pelinson, 2004; Alcaniz and Lima, 2005; Opher and Pelinson, 2005; Wang and Meng, 2005; Fabris et al., 2007; Jesus et al., 2008; Costa et al., 2008; Basilakos et al., 2009; Grande et al., 2011; Xu et al., 2011; Basilakos et al., 2012].

Os modelos com energia escura dinâmica atuam eficientemente ao atenuar, não apenas o problema da constante cosmológica mas também o problema da coincidência, já que a conservação do tensor 
momento energia total do universo obriga aos fluidos materiais a receberem a energia que vem do decrescimento na densidade do vácuo - nos casos em que este último ocorre - fornecendo uma ligação entre as densidades de energia material e do vácuo. Em geral, os modelos com uma densidade do vácuo que decresce ao longo da historia cósmica possuem as seguintes propriedades:

i) Sugerem um novo mecanismo de inflação gerada pelo domínio primordial da densidade do vácuo, ii) apresentam uma saída natural (graceful exit problem) para um universo quente dominado por radiação e permitindo o posterior domínio da matéria escura fria, iii) mantém uma conexão entre as densidades de energia da componente material e do vácuo ao longo dos períodos desacelerados cuja consequência é aliviar o problema da coincidência, iv) e permitem um estágio final dominado por uma densidade do vácuo remanescente, gerando assim o estágio final acelerado, recentemente observado. Tais modelos possuem todas as vantagens do modelo inflação $+\Lambda \mathrm{CDM}$, reduzindo o setor escuro+inflacionário, ligando ainda os estágios inicial e final acelerados, atenuando os problemas da constante cosmológica e da coincidência, e sendo compatíveis com os resultados das observações cosmológicas do universo recente [Lima and Maia, 1994; Lima and Trodden, 1996; Perico et al., 2013a]. Esta série de características constituem a nossa motivação para analisar as consequências desta classe de modelos cosmológicos.

Sendo a variação no valor esperado do vácuo uma consequência universal da teoria quântica de campos, compatível com os postulados da relatividade geral, e sabendo que sua presença no universo pode evitar a singularidade primordial, faz sentido perguntarmos por suas consequências nos estágios finais do processo de colapso gravitacional [Basilakos et al., 2010; Campos and Lima, 2012]. Um dos objetivos deste trabalho foi caracterizar os efeitos da variação na densidade do vácuo, no caso em que este evolui com a taxa de contração do sistema, durante os estágios finais do processo de colapso gravitacional. Em particular, fizemos uma análise detalhada para determinar se a formação de uma singularidade e de um buraco negro podem ser evitadas pelo presença deste tipo de vácuo dinâmico.

Para descrever tais resultados, a presente tese foi organizada da seguinte forma:

- No capítulo 2 apresentamos brevemente o modelo de universo homogêneo e isotrópico em expansão descrito pela métrica FRW. Em seguida, utilizando as equações de Einstein para um universo composto por fluidos ideais, discutimos como evoluem as densidades de energia médias da radiação, da matéria escura e do vácuo não interagente em função do fator de escala do universo. A evolução do fator de escala em função do tempo para os diferentes períodos de domínio de cada uma das componentes principais do modelo $\Lambda \mathrm{CDM}$ é também apresentada.

- No capítulo 3 - fazendo uso da teoria de perturbações lineares aplicável ao universo em grandes escalas (maiores que 100 Mpc [Mukhanov, 2005]) onde as inomogeneidades e anisotropias na densidade de energia são muito menores que a densidade média - introduziremos perturbações tanto na métrica quanto no tensor momento energia (na aproximação de fluidos ideais do conteúdo material). Centraremos nossa análise nas perturbações escalares, responsáveis pela formação de estruturas devido a sua natureza aglomerativa. Chegaremos às equações linearizadas de Einstein e de conservação do tensor momento energia que regem a evolução das perturbações no fundo homogêneo e isotrópico estudado no capítulo 2.

- No capítulo 4 estudamos o problema de horizonte existente nos modelos tipo big-bang quente como uma motivação para introduzir o período inflacionário. Apresentamos a popular implementação 
do período inflacionário gerado por meio de um campo escalar, e discutimos a evolução das perturbações tanto do campo escalar inflacionário, como das perturbações escalares e tensoriais da métrica.

- No capítulo 5 analisamos de uma forma geral a expansão de universos com uma densidade do vácuo que cresce monotonamente com o parâmetro de Hubble, caracterizando aqueles que apresentam eras iniciais e finais assintoticamente de Sitter. Na sequência, algumas propostas fenomenológicas para este tipo de modelos são estudadas. Por fim, usando as ideias fundamentais do grupo de renormalização, apresentaremos uma justificativa para a variação do valor esperado do vácuo com a escala de energia característica do sistema em questão. Isto permitirá aliviar os problemas da constante cosmológica e da coincidência, e em alguns casos consegue reproduzir o estágio primordial acelerado, reduzindo o setor inflacionário.

- No capítulo 6 realizamos uma análise detalhada da história cósmica para um universo com uma densidade do vácuo que possui um termo constante e um outro termo que cresce mais rapidamente que o quadrado do parâmetro de Hubble. Na aproximação de um universo perfeitamente homogêneo e isotrópico mostraremos explicitamente como são atenuados o problema da constante cosmológica e o problema da coincidência. Também apresentaremos alguns testes cosmológicas deste modelo, incluindo o nível perturbativo, tanto para o universo recente como para o estágio primordial acelerado. Tais análises permitirão obter estimativos para os parâmetros livres do modelo.

- Resultados da compatibilidade do modelo de vácuo dinâmico com a segunda lei da termodinâmica são apresentados no capítulo 7, mostrando que tanto o período inflacionário como os períodos de domínio da radiação e da matéria escura não conduzem o universo para o equilíbrio termodinâmico, enquanto que o período final acelerado o conduz para um estado com entropia máxima.

- No capítulo 8 mostramos como os resultados dinâmicos obtidos para o modelo plano $(\kappa=0)$ podem ser generalizados para curvaturas arbitrárias. O interesse deste resultado está associado ao fato de que as coordenadas utilizadas para descrever o universo plano (de Sitter) cobrem apenas uma parte do hiperboloide de Sitter.

- No capítulo 9 investigamos as consequências da presença de uma densidade do vácuo que cresce de forma proporcional à densidade total de um núcleo estelar sofrendo o processo final de colapso. Veremos que a formação de um estado final singular e de um horizonte aparente em volta dele não são afetados pela presença de curvatura espacial. Também quantificaremos as principais previsões referentes ao tempo de colapso, tempo formação do horizonte, também como a massa contida dentro do horizonte aparente, levando em conta que a geometria espacial pode ser aberta, fechada ou plana.

- No capítulo 10 discutimos as consequências da presença de uma densidade do vácuo que domina um núcleo estelar no regime de altas energias, durante os estágios finais do processo de colapso gravitacional. Mostramos que em esta classe de modelos, a formação de um estado final singular é evitada.

- As considerações finais do trabalho são apresentadas no capítulo 11. 
Nosso contato com a literatura indica que os resultados originais de esta monografia se encontram nos capítulos 5-10. Em particular, os resultados do capítulo 6, referentes ao cenário cosmológico plano no nível não perturbado, foram publicados na referência [Perico et al., 2013a]. Já os resultados dos testes cosmológicos referentes a história cósmica e ao espectro de potências da matéria (também apresentados no mesmo capítulo) serão submetidos à publicação [Perico and Lima, 2015]. Os resultados do capítulo 8 referentes ao estágio primordial acelerado do modelo deflacionário com curvatura espacial já foram publicados e estão contidos na referência [Lima et al., 2015b]. Por outro lado, os resultados referentes ao processo de colapso gravitacional incluindo curvatura espacial, apresentados no capítulo 9, estão submetidos à publicação [Perico et al., 2013b]. Finalmente, os resultados sobre a possibilidade de se evitar o estado singular final no processo de colapso gravitacional, devido a ação de termos de ordem superior, tal como descritos no capítulo 10, serão brevemente submetidos à publicação [Lima et al., 2015a].

Resta dizer que ao longo do trabalho usaremos indistinguivelmente os conceitos de horizonte atrapante e horizonte aparente (ver apêndice A), já que nos sistemas estudados neste trabalho o escalar de Ricci é sempre positivo, e foi mostrado por [Faraoni, 2011] que um horizonte aparente é atrapante sempre que o escalar de Ricci seja positivo. Ao longo de todo o trabalho usaremos a notação descrita em nomenclatura, como também usaremos unidades de $\hbar=c=1$, excepto no capítulo 7 . 



\section{COSMOLOGIAS HOMOGÊNEAS E ISOTRÓPICAS}

Neste capítulo introduziremos os elementos que são a base do modelo cosmológico padrão, o modelo $\Lambda$ CDM. O eixo central deste modelo de universo é a relatividade geral, condensada na equação de Einstein [Carroll, 2004], enquanto que seu conteúdo material esta composto principalmente por fótons $(\gamma)$, matéria escura fria ou não relativística (CDM) e energia escura, representada pela constante cosmológica $(\Lambda)$.

Antes de introduzirmos a equação de Einstein (que relaciona dinamicamente a geometria do universo com seu conteúdo energético) apresentaremos alguns elementos de geometria diferencial necessários para manipular e interpretar a modelagem geométrica da gravidade feita através desta equação. Posteriormente iremos descrever o cenário base do nosso modelo: um universo espacialmente plano em expansão, aproximadamente homogêneo e isotrópico em grandes escalas (da ordem de 100Mpc que é a distância típica entre aglomerados de galáxias [Mukhanov, 2005]). Logo depois discutiremos a modelagem mais simples do conteúdo energético do universo (fluidos ideais não interagentes) no modelo

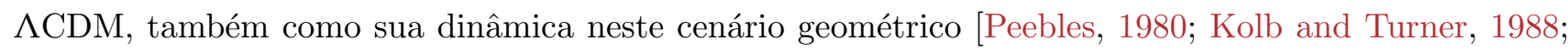
Peacock, 1999; Narlikar, 2002; Dodelson, 2003].

O modelo matemático para a geometria do universo interagindo com seu conteúdo energético nos levará às expressões que descrevem a evolução da densidade de energia média das componentes dominantes (radiação, matéria escura e constante cosmológica) em função do fator de escala do universo $a$. Com isto será possível descrever a evolução temporal do fator de escala durante os períodos de domínio de cada um desses fluidos.

\subsection{GEOMETRIA DO UNIVERSO}

Como veremos nesta seção, a métrica é o objeto geométrico que define univocamente (para espaçotempos sem torção) a estrutura causal de todo sistema gravitacional em relatividade geral, ela é a estrutura fundamental que nos permite medir comprimentos de curvas e magnitudes de vetores. Por exemplo, a medida do comprimento $s$ de uma curva pode ser feita pela integração do seu elemento de linha ds definido como:

$$
d s^{2}=g_{\mu v} d x^{\mu} d x^{v},
$$

onde $g_{\mu \nu}$ é chamado de tensor métrico. Este é um tensor de grau $(0,2)$ simétrico, $g_{\mu \nu}=g_{v \mu}$, e com determinante diferente de zero, esto permite que seja invertido se necessário $\left(g^{\mu v} g_{v \sigma}=g_{\lambda \sigma} g^{\lambda \mu}=\delta_{\sigma}^{\mu}\right)$. O tensor métrico não é apenas utilizado para calcular escalares simples como magnitudes, também pode ser empregado na determinação da causalidade do espaço tempo, permitindo calcular as trajetórias das partículas livres, ou mesmo descrever uma fonte de gravidade. 
Com a forma de medir distâncias esclarecida, se define a derivada covariante como sendo uma derivada que transforma tensores de grau $N$ em tensores de grau $N+1$, e que se reduz à derivada parcial sobre uma variedade plana em coordenadas inerciais. Escrevemos, então, a derivada covariante de um tensor de grau $(i, j)$ como sendo:

$$
\begin{aligned}
& T_{v_{1} v_{2} \ldots v_{j} ; \sigma}^{\mu_{1} \mu_{2} \ldots \mu_{i}}-T_{v_{1} v_{2} \ldots v_{j}, \sigma}^{\mu_{1} \mu_{2} \ldots \mu_{i}}=\Gamma_{\sigma \lambda}^{\mu_{1}} T_{v_{1} v_{2} \ldots n u_{j}}^{\lambda \mu_{2} \ldots \mu_{i}}+\Gamma_{\sigma \lambda}^{\mu_{2}} T_{v_{1} v_{2} \ldots v_{j}}^{\mu_{1} \lambda \mu_{3} \ldots \mu_{i}}+\cdots+\Gamma_{\sigma \lambda}^{\mu_{i}} T_{v_{1} v_{2} \ldots v_{j}}^{\mu_{1} \mu_{2} \ldots \mu_{i-1} \lambda} \\
& -\Gamma_{\sigma v_{1}}^{\lambda} T_{\lambda v_{2} \ldots v_{j}}^{\mu_{1} \mu_{2} \ldots \mu_{i}}-\Gamma_{\sigma v_{2}}^{\lambda} T_{v_{1} \lambda v_{3} \ldots v_{j}}^{\mu_{1} \mu_{2} \ldots \mu_{i}}-\cdots-\Gamma_{\sigma v_{j}}^{\lambda} T_{v_{1} v_{2} \ldots \nu_{j-1} \lambda}^{\mu_{1} \mu_{2} \ldots \mu_{i}},
\end{aligned}
$$

onde o ponto e vírgula denotam a derivada covariante, enquanto que a vírgula denota a derivada parcial. Os símbolos $\Gamma_{v \alpha}^{\mu}$ (chamados de conexão) não são tensores, e a derivada parcial de um tensor também não é em geral um tensor, mas a soma (2.2) é definida de modo a ser um tensor. Por outro lado, a conexão depende da curvatura da variedade (a conexão é intrínseca à variedade), mas pode ou não depender da métrica. No caso da relatividade geral, se utiliza a única conexão que é compatível com a métrica (ou seja, aquela que permite $g_{\mu v ; \sigma}=0$ ) e que não possui torção (isto é $\Gamma_{\alpha \beta}^{\mu}=\Gamma_{\beta \alpha}^{\mu}$ ). A conexão em relatividade geral pode ser escrita como:

$$
\Gamma_{\alpha \beta}^{\mu}=\frac{1}{2} g^{\mu v}\left(g_{\alpha v, \beta}+g_{\beta v, \alpha}-g_{\alpha \beta, v}\right)
$$

Um conceito fundamental em geometria diferencial é o processo de transporte paralelo, que nasce da necessidade de comparar dois tensores que inicialmente estão definidos em diferentes pontos da variedade. Para comparar dois vetores, primeiramente devemos transportá-los até um mesmo ponto. Assim, o transporte paralelo é definido simplesmente como o transporte que conserva constantes cada uma das componentes de um tensor em relação ao vetor tangente à trajetória de transporte, o que em coordenadas curvilíneas em espaços planos não produz um resultado trivial, já que os versores da base podem variar ponto a ponto da variedade. Devido à presença de curvatura numa variedade arbitrária o transporte paralelo de um tensor depende da trajetória do transporte, a diferença de uma variedade plana.

A interpretação geométrica da derivada covariante pode ser feita no contexto do transporte paralelo: a derivada covariante $T_{; \sigma}(\lambda)(2.2)$ de um tensor $T(\lambda)$ é sua taxa instantânea de variação, na direção do vetor $d x^{\sigma} / d \lambda$, em relação ao tensor $T^{\prime}(\lambda)$ gerado a partir do transporte paralelo do tensor inicial ao longo da curva $\gamma(\lambda)$ descrita pelas coordenadas $x^{\mu}(\lambda)$. Deste modo, as componentes do tensor $T_{\nu_{1} v_{2} \ldots v_{j}}^{\mu_{1} \mu_{2} \ldots \mu_{i}}$ transportado paralelamente ao longo da curva $x^{\sigma}$ (parametrizada pelo parâmetro continuo $\lambda$ ) respeitam a relação:

$$
\left(\frac{D}{d \lambda} T\right)_{v_{1} v_{2} \ldots \nu_{j}}^{\mu_{1} \mu_{2} \ldots \mu_{i}} \equiv \frac{d x^{\sigma}}{d \lambda} T_{\nu_{1} \nu_{2} \ldots \nu_{j} ; \sigma}^{\mu_{1} \mu_{2} \ldots \mu_{i}}=0
$$

e, para o caso de um vetor $V^{\sigma}$, assume a forma simples:

$$
\frac{d V^{\sigma}}{d \lambda}+\Gamma_{\alpha \beta}^{\sigma} \frac{d x^{\alpha}}{d \lambda} V^{\beta}=0
$$


Fazendo o transporte paralelo do vetor $V^{\sigma} \equiv \frac{d x^{\sigma}}{d \lambda}$ (é o vetor tangente à curva $x^{\sigma}$ ) ao longo da curva descrita pelo mesmo $x^{\sigma}(\lambda)$, obtemos a conhecida equação geodésica:

$$
\frac{d^{2} x^{\mu}}{d \lambda^{2}}+\Gamma_{\alpha \beta}^{\mu} \frac{d x^{\alpha}}{d \lambda} \frac{d x^{\beta}}{d \lambda}=0
$$

que descreve a trajetória clássica de uma partícula livre na variedade. Em geral a equação geodésica descreve um vetor $V^{\mu}$ tal que ele é transportado de forma paralela ao longo de sua própria curva integral, o que pode ser escrito como:

$$
V_{; \nu}^{\mu} V^{v}=f V^{\mu}
$$

Existe o chamado parâmetro afim $\lambda$, tal que a função $f(\lambda)=0$, fazendo com que as componentes da equação geodésica (2.7) fiquem descritas pela equação (2.6).

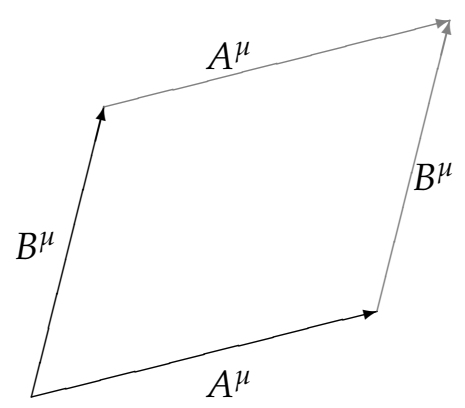

Figura 2.1: Laço infinitesimal definido pelos vetores $A^{\mu}$ e $B^{\mu}$.

Se agora fizermos o transporte paralelo do vetor $V^{\alpha}$ ao longo do caminho infinitesimal fechado descrito pelos vetores A e B (figura 2.1), e no sentido anti-horário, o vetor vai experimentar a mudança:

$$
\delta V^{\alpha}=R_{\beta \mu \nu}^{\alpha} V^{\beta} A^{\mu} B^{v},
$$

o que fornece a definição do tensor de curvatura de Riemann $R_{\beta \mu v}^{\alpha}$. Na ausência de torção, podemos ainda escrevê-lo como:

$$
R_{\beta \mu v}^{\alpha}=\Gamma_{\nu \beta ; \mu}^{\alpha}-\Gamma_{\mu \beta ; v}^{\alpha}+\Gamma_{\mu \lambda}^{\alpha} \Gamma_{\nu \beta}^{\lambda}-\Gamma_{\nu \lambda}^{\alpha} \Gamma_{\mu \beta}^{\lambda},
$$

e quando contraído com a métrica resulta no chamado tensor de Ricci:

$$
R_{\mu v}=R_{\mu \lambda \nu}^{\lambda}=g_{\beta}^{\alpha} R_{\mu \beta v}^{\alpha}
$$

se contraído mais uma vez com a métrica, obtemos o chamado escalar curvatura:

$$
\mathcal{R}=g^{\mu v} R_{\mu v}
$$

Este escalar é essencial na definição da ação geométrica cuja variação da origem à equação de Einstein. Lembremos ainda que os escalares (tensores de ordem $(0,0)$ ) são invariantes, isto é, não dependem do sistema de coordenadas inercial escolhido. 

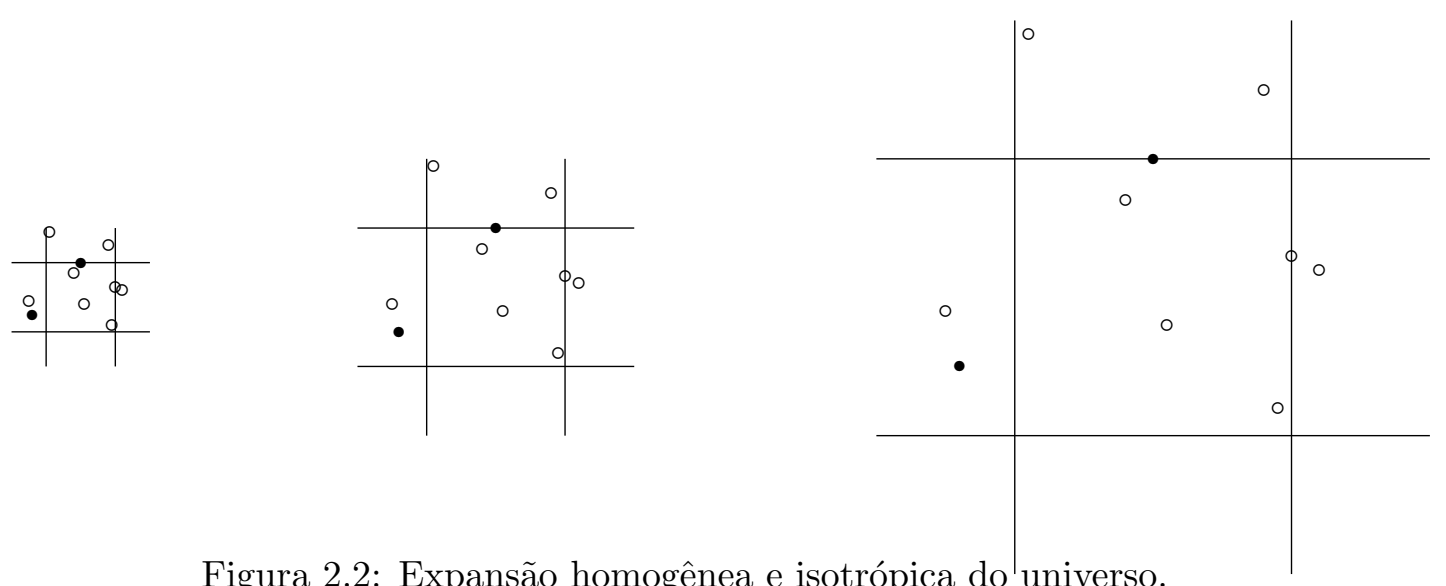

Figura 2.2: Expansão homogênea e isotrópica do universo.

Da esquerda para a direita mostramos a mesma configuração de galáxias distantes para os tempos $\eta_{1}<\eta_{2}<\eta_{3}$. A distância comóvel $d_{c}$ entre as duas galáxias pretas independe do tempo. Enquanto que a distância própria entre elas aumenta de forma proporcional ao fator de escala para cada instante de tempo: $d_{p}\left(\eta_{1}\right)=a\left(\eta_{1}\right) d_{c}, \quad d_{p}\left(\eta_{2}\right)=a\left(\eta_{2}\right) d_{c} \quad$ e $d_{p}\left(\eta_{3}\right)=a\left(\eta_{3}\right) d_{c} \quad$ com $a\left(\eta_{1}\right)<a\left(\eta_{2}\right)<a\left(\eta_{3}\right)$ [Dodelson, 2003].

\subsubsection{Expansão do universo}

Para um universo em expansão uniforme, a distância física entre duas galáxias distantes aumenta em função do tempo, ou seja, galáxias distantes estão se afastando umas das outras. Se o universo está expandindo uniformemente, podemos traçar uma rede imaginária que cresce uniformemente com o universo, como mostrado na figura 2.2. Chamaremos às coordenadas que descrevem esta rede como coordenadas comoveis; e à distância em relação a tais coordenadas, a chamaremos de distância comóvel. Assim definida, a distância comóvel entre duas galáxias distantes não muda em função do tempo. Porém, a distância física entre dois pontos no universo é igual à distância comóvel multiplicada por um fator de escala, que chamamos de $a \equiv a(t)$, e que cresce em função do tempo. Com isto, a relação entre as coordenadas comoveis $x^{\mu}=(\eta, \mathbf{x})$ e as coordenadas físicas $r^{\mu}=(t, \mathbf{r})$ de um ponto no universo em expansão uniforme é:

$$
r^{\mu}(x, t)=x^{\mu} a(t)
$$

e a velocidade física de uma galáxia situada na coordenada física $\mathbf{r}$ esta dada por:

$$
\frac{d \mathbf{r}}{d t}=\frac{d \mathbf{x}}{d t} a+\mathbf{x} \frac{d a}{d t} .
$$

Para uma de velocidade peculiar nula $(d \mathbf{x} / d t=0$, estaticidade em relação à rede comóvel) a velocidade física será:

$$
\frac{d \mathbf{r}}{d t}=\frac{1}{a} \frac{d a}{d t} a \mathbf{x}=\frac{1}{a} \frac{d a}{d t} \mathbf{r},
$$

que corresponde à chamada lei de Hubble. Se o universo está expandindo uniformemente (todos os pontos nele estão se afastando uns dos outros) a lei de Hubble prediz que a velocidade instantânea de 
afastamento das galáxias distantes aumenta linearmente com a distância ao observador (2.14), onde a constante de proporcionalidade $H$ é conhecida como parâmetro de Hubble, e pode ser escrita como:

$$
H(t) \equiv \frac{1}{a} \frac{d a}{d t}=\frac{1}{a^{2}} \frac{d a}{d \eta} \equiv \frac{\dot{a}}{a^{2}} \equiv a \mathcal{H},
$$

onde o ponto denota a derivada em relação ao tempo conforme $\eta$, definido como $d t=a d \eta$ ( $t$ é o tempo físico). Utilizando os conceitos apresentados até agora, podemos introduzir a métrica para um universo homogêneo, isotrópico, espacialmente plano e em expansão, que escrita em coordenadas comoveis esféricas usando o tempo conforme esta dada por

$$
d s^{2}=g_{\mu \nu} d x^{\mu} d x^{v}=a^{2}\left(-d \eta^{2}+d r^{2}+r^{2} d \Omega^{2}\right)=-d t^{2}+a^{2}\left(d r^{2}+r^{2} d \Omega^{2}\right)
$$

onde $d \Omega^{2} \equiv d \theta^{2}+\sin ^{2} \theta d \varphi^{2}$ é o elemento de área para uma 2-esfera unitária. Contudo, existe um caso mais geral e muito mais interessante: a chamada métrica de Friedmann-Robertson-Walker (FRW), que descreve de forma geral uma variedade Lorentziana estacionária com simetria espacial maximal, e que usaremos para modelar a geometria de um universo homogêneo, isotrópico e em expansão uniforme com curvatura espacial positiva (universo fechado), negativa (universo aberto) ou nula (universo plano). A métrica FRW pode ser escrita em coordenadas esféricas como [Weinberg, 1972]:

$$
d s^{2}=g_{\mu v} d x^{\mu} d x^{\nu}=a^{2}\left(-d \eta^{2}+\frac{d r^{2}}{1-\kappa r^{2}}+r^{2} d \Omega^{2}\right)=-d t^{2}+a^{2}\left(\frac{d r^{2}}{1-\kappa r^{2}}+r^{2} d \Omega^{2}\right)
$$

onde $\kappa>0, \kappa<0$ ou $\kappa=0$ descrevem um universo espacialmente fechado, aberto ou plano respectivamente. As componentes não nulas da conexão (2.3) para esta métrica são:

$$
\begin{array}{llll}
\Gamma_{00}^{0}=\frac{\dot{a}}{a}, & \Gamma_{11}^{0}=\frac{\dot{a}}{a}\left(\frac{1}{1-\kappa r^{2}}\right), & \Gamma_{22}^{0}=\frac{\dot{a}}{a} r^{2}, & \Gamma_{33}^{0}=\Gamma_{22}^{0} \sin ^{2} \theta, \\
\Gamma_{10}^{1}=\frac{\dot{a}}{a}, & \Gamma_{11}^{1}=\frac{\kappa r}{1-\kappa r^{2}}, & \Gamma_{22}^{1}=-r\left(1-\kappa r^{2}\right), & \Gamma_{33}^{1}=\Gamma_{22}^{1} \sin ^{2} \theta, \\
\Gamma_{20}^{2}=\frac{\dot{a}}{a}, & \Gamma_{21}^{2}=\frac{1}{r}, & & \Gamma_{33}^{2}=-\cos \theta \sin \theta, \\
\Gamma_{30}^{3}=\frac{\dot{a}}{a}, & \Gamma_{31}^{3}=\frac{1}{r}, & \Gamma_{32}^{3}=\cot \theta, &
\end{array}
$$

enquanto que as componentes não nulas do tensor de Ricci (2.10) são

$$
\begin{array}{ll}
R_{00}=3\left[\left(\frac{\dot{a}}{a}\right)^{2}-\frac{\ddot{a}}{a}\right]_{2}, & R_{11}=\frac{1}{1-\kappa r^{2}}\left[2 \kappa+\left(\frac{\dot{a}}{\bar{a}}\right)^{2}+\frac{\ddot{a}}{a}\right] \\
R_{22}=r^{2}\left[2 \kappa+\left(\frac{\dot{a}}{a}\right)^{2}+\frac{\ddot{a}}{a}\right], & R_{33}=r^{2} \sin ^{2} \theta\left[2 \kappa+\left(\frac{\dot{a}}{\bar{a}}\right)^{2}+\frac{\ddot{a}}{a}\right],
\end{array}
$$

e o escalar de Ricci (2.11) é:

$$
\mathcal{R}=\frac{6}{a^{2}}\left(\kappa+\frac{\ddot{a}}{a}\right)
$$




\section{Horizonte comóvel}

Para uma partícula que viaja seguindo uma geodésica nula em um universo espacialmente plano $(\kappa=0)$ temos

$$
d s^{2}=0=a^{2}(t)\left(-d \eta^{2}+d x^{2}\right), \quad \text { assim } \quad d x= \pm d \eta,
$$

desta forma, a máxima distância comóvel que pode viajar um fóton desde o começo da história do universo, $\eta=0$, até o instante de tempo $\eta$ é igual ao tempo conforme $\eta$. Se dois pontos no espaço tempo estão afastados por uma distância maior que $\eta$ nunca estiveram em contato causal, já que $\eta$ representa a distância máxima que um fóton pode viajar (a velocidade $c=1$ ) desde o início do universo. Portanto, o tempo conforme $\eta$ também é chamado de horizonte comóvel.

\section{Raio comóvel de Hubble}

Mais para frente veremos que em um universo plano dominado por um fluido com equação de estado $\mathcal{P}=\omega \rho$ o fator de escala evolui na forma

$$
a=c_{\omega} t^{2 /(3(1+\omega))} \text { para } \omega \neq-1, \text { e } H=\text { cte para } \omega=-1,
$$

onde $c_{\omega}$ é uma constante. Assim, a máxima distância comóvel $\Delta \eta$ que consegue percorrer um fóton enquanto o fator de escala se duplica é igual a

$$
\begin{gathered}
\Delta \eta=\int_{a_{1}}^{2 a_{1}} d \eta=\int_{a_{1}}^{2 a_{1}} \frac{d t}{a}=\left.\frac{2}{1+3 \omega} \frac{1}{a H}\right|_{a_{1}} ^{2 a_{1}}=\frac{2}{1+3 \omega} \frac{1}{a_{1} H_{1}}\left(2^{\frac{1+3 \omega}{3(1+\omega)}}-1\right), \quad \text { para } \omega \neq 1, \\
\Delta \eta=\int_{a_{1}}^{2 a_{1}} \frac{d \eta}{d a} d a=\int_{a_{1}}^{2 a_{1}} \frac{d a}{a^{2} H}=\left.\frac{-1}{a H}\right|_{a_{1}} ^{2 a_{1}}=\frac{1}{H_{1}} \int_{a_{1}}^{2 a_{1}} \frac{d a}{a^{2}}=\frac{1}{2 a_{1} H_{1}}, \quad \text { para } \quad \omega=-1,
\end{gathered}
$$

e portanto

$$
\Delta \eta=\left\{\frac{1}{2 a H}, \frac{1}{1.92366 a H}, \frac{1}{2.4121 a H}\right\} \quad \text { para } \omega=\left\{-1,0, \frac{1}{3}\right\} .
$$

Com isso, o raio comóvel de Hubble (definido como $a / \dot{a} \equiv \mathcal{H}^{-1}$ ) é aproximadamente o dobro da máxima distância comóvel que pode viajar um fóton enquanto o fator de escala se duplica. Assim, dois pontos separados por uma distância menor que o raio de Hubble podem se comunicar eficientemente em comparação com a taxa de expansão do universo. Para uma região com comprimento característico igual ou menor ao raio comóvel de Hubble, um fluido pode chegar a estabelecer um equilíbrio termodinâmico mesmo quando o universo se expande. Analogamente $H^{-1}$ é conhecido como raio de Hubble.

\section{Horizonte de partículas}

Rm um espaço-tempo arbitrário, o horizonte de partículas cosmológico de um observador $q$ viajando em uma linha de mundo $\mathcal{O}$ (uma geodésica temporal), tal que $\mathcal{O}\left(t=t_{q}\right)=q$, consiste na superfície que divide as linhas de mundo das partículas que já foram observadas por $\mathcal{O}$ até $t=t_{q}$, das linhas de mundo das partículas que ainda não foram observados por $\mathcal{O}$ até $t=t_{q}$, portanto, o horizonte de partículas depende do observador e da estrutura causal do espaço-tempo. 
Para um espaço-tempo com uma curvatura constante a estrutura causal não depende do ponto de observação (os cones de luz possuem a mesma forma sobre toda a variedade) mas para diferentes instantes de tempo, uma mesma linha de mundo $\mathcal{O}$ pode observar horizontes de partículas maiores quanto mais avança o tempo, já que seus cones de luz se estendem mais longe com o decorrer do tempo. Para uma espaço-tempo com uma métrica não homogênea os cones de luz dependem do ponto do espaço-tempo, e portanto o horizonte de partículas depende também da linha de mundo do observador como do instante de observação. Para um espaço-tempo $(\mathcal{M}, g)$ com um infinito nulo passado $\mathscr{I}^{-}$do tipo nulo ou temporal, o horizonte de partículas para todo $q \in \mathcal{M}$ é infinito (não existe um horizonte de partículas). Mas, para um espaço-tempo $\left(\mathcal{M}^{\prime}, g^{\prime}\right)$ com um infinito nulo passado $\mathscr{I}^{-}$do tipo espacial, o horizonte de partículas é finito.

\section{Horizonte cosmológico de eventos futuros}

O horizonte de ventos para um observador cosmológico com uma linha de mundo $\mathcal{O}$, portanto do tipo temporal, é a superfície que separa todos os eventos que foram, estão sendo ou que serão observados por $\mathcal{O}$, dos eventos que nunca serão observados por $\mathcal{O}$.

Para um espaço-tempo com um infinito nulo futuro $\mathscr{I}^{+}$do tipo espaço, o conjunto de eventos que $\mathcal{O}$ nunca observará é diferente de zero, e portanto possui um horizonte de eventos futuro. Por outro lado, se o infinito nulo futuro $\mathscr{I}^{+}$é do tipo nulo ou temporal, um observador sobre uma linha de mundo que acabe no infinito temporal futuro $i^{+}$conseguira ver todos os eventos no espaço-tempo ao longo de sua trajetória.

Analogamente pode-se definir o horizonte de eventos passados para uma linha de mundo do tipo tempo.

\subsection{DINÂMICA DO UNIVERSO EM GRANDES ESCALAS}

A relatividade geral é uma teoria que descreve a forma como a curvatura do espaço-tempo (informação que podemos obter a partir do tensor métrico) atua sobre a matéria para se manifestar como gravidade, e como a energia e o momento dos fluidos contidos no universo determinam a curvatura do espaço-tempo. A equação fundamental desta teoria é a equação de Einstein (2.26), que relaciona dinamicamente duas componentes do universo: o tensor momento energia $T_{\mu v}$ que da conta do conteúdo energético do universo, e o tensor de Einstein (lado esquerdo da equação (2.26)) que contém a informação sobre a geometria

$$
R_{\mu \nu}-\frac{1}{2} \mathcal{R} g_{\mu \nu}=8 \pi G T_{\mu \nu}
$$

Esta equação pode ser obtida impondo que a variação da ação $S$ em relação à métrica $g^{\mu v}$ seja nula:

$$
S=\frac{1}{16 \pi G} \int d^{4} x \sqrt{-g} \mathcal{R}+S_{M}, \quad \delta S=0,
$$

onde $S_{M}$ é a ação associada ao conteúdo energético do universo, e dá origem ao tensor momento energia

$$
T_{\mu v}=-2 \frac{1}{\sqrt{-g}} \frac{\delta S_{M}}{\delta g^{\mu \nu}} .
$$


Para um fluido ideal que se expande livremente dentro de um universo descrito pela métrica $g^{\mu \nu}$, o tensor momento energia pode ser escrito na forma:

$$
T^{\mu v}=\mathcal{P} g^{\mu v}+(\rho+\mathcal{P}) U^{\mu} U^{v},
$$

onde $\rho, \mathcal{P}$ e $U$ são, respetivamente, a densidade de energia, a pressão e a 4-velocidade do fluido, todas medidas por um mesmo observador. Para um universo composto por uma mistura de $M$ fluidos, que podem ou não interagir entre eles, o tensor momento energia total é descrito por:

$$
T^{\mu v}=\sum_{x=1}^{M}\left(\mathcal{P}_{x} g^{\mu v}+\left(\rho_{x}+\mathcal{P}_{x}\right) U_{x}^{\mu} U_{x}^{v}\right)
$$

onde $U_{x}^{\mu}, \rho_{x}$ e $\mathcal{P}_{x}$ são, respetivamente, a 4 -velocidade, a densidade de energia e a pressão do $x$-ésimo fluido.

Em particular, dado que nosso interesse é estudar a evolução do conteúdo energético do universo em grandes escalas, onde todas as grandezas físicas observadas são aproximadamente isotrópicas e homogêneas, vamos modelar a geometria do universo pela métrica FRW (2.16). No seguinte capítulo consideraremos perturbações tanto na métrica como na pressão, na densidade de energia, na velocidade e na componente anisotrópica do tensor momento energia de cada fluido. Com esta aproximação, um observador comóvel mede em qualquer ponto do espaço uma densidade de energia e uma pressão iguais a suas médias espaciais $\left(\rho_{x}(t)=\bar{\rho}_{x}(t)\right)$-em geral diferentes para cada fluido- e velocidades peculiares nulas, $U_{x}^{\mu}=(1, \mathbf{0}) \forall x$. Para o dito observador, o tensor momento energia (2.29) se reduz a:

$$
T_{v}^{\mu}=\left(\begin{array}{cccc}
-\rho_{T} & 0 & 0 & 0 \\
0 & \mathcal{P}_{T} & 0 & 0 \\
0 & 0 & \mathcal{P}_{T} & 0 \\
0 & 0 & 0 & \mathcal{P}_{T}
\end{array}\right)
$$

onde $\rho_{\mathrm{T}} \equiv \sum_{x=1}^{M} \rho_{x}$ e $\mathcal{P}_{\mathrm{T}} \equiv \sum_{x=1}^{M} \mathcal{P}_{x}$ são a densidade de energia total e a pressão total no sistema de coordenadas comóvel comum para todas as componentes da mistura.

No contexto da relatividade geral a derivada covariante do tensor métrico é definida como sendo nula, $g_{; \mu}^{\mu v} \equiv 0$, desta forma a derivada covariante da parte geométrica (lado esquerdo da equação de Einstein (2.26)) se anula, já que está escrita em função do tensor métrico e de suas primeiras e segundas derivadas parciais. Esta equação é conhecida como a identidade de Bianchi. Como consequência, a derivada covariante do tensor momento energia total (lado direito da equação de Einstein) também é nula, $T_{; \mu}^{\mu \nu}=0$, se assumirmos a imutabilidade da constante de gravitação universal G. Esta equação é conhecida como a lei de conservação covariante do tensor momento energia total:

$$
T_{; \mu}^{\mu v}=\partial_{\mu} T^{\mu v}+\Gamma_{\alpha \beta}^{v} T^{\alpha \beta}+\Gamma_{\alpha \beta}^{\alpha} T^{\nu \beta}=0 .
$$


No caso de um universo descrito pela métrica FRW (2.16) e composto por uma mistura de $M$ fluidos, com tensor momento energia total dado por (2.30), a componente temporal da equação de conservação fica expressa como:

$$
\sum_{x=1}^{M}\left(\dot{\rho}_{x}+3 \mathcal{H}\left(\rho_{x}+\mathcal{P}_{x}\right)\right)=0, \quad \text { ou } \quad \sum_{x=1}^{M}\left(\rho_{x}^{\prime}+3 \frac{\rho_{x}+\mathcal{P}_{x}}{a}\right)=0,
$$

enquanto a componente espacial $(v=i)$ é identicamente nula, esta é uma consequência do modelo perfeitamente homogêneo e isotrópico que estamos considerando neste capitulo. Quando incluirmos perturbações, tanto no tensor métrico como no tensor momento energia, esta equação torna-se não trivial.

Ao longo deste trabalho estaremos interessados na modelagem do universo composto pela mistura de dois fluidos; um fluido material (cuja densidade de energia e pressão, que denotaremos como simplesmente $\rho$ e $\mathcal{P}$-sem subíndices-, estão relacionadas pela equação de estado $\mathcal{P}=\omega \rho$ com $\omega \geqslant 0$ ) e o vácuo (com densidade $\rho_{\Lambda} \equiv \Lambda /(8 \pi G)$ e equação de estado $\left.\mathcal{P}_{\Lambda}=-\rho_{\Lambda}\right)$. Estas duas densidades de energia podem variar em função do tempo, enquanto o parâmetro da equação de estado $\omega$ permanece constante para cada uma das componentes. Por sua vez, o fluido material será aproximado como sendo uma mistura de fluidos relativísticos (com equação de estado $\mathcal{P}=\rho / 3$, ou seja, $\omega=1 / 3$ ) e matéria escura fria (com $\omega=0$ ou pressão nula). Por simplicidade, vamos nos concentrar em casos onde uma das componentes materiais domina sobre a outra. Mais especificamente, o fluido material dominante será descrito como sendo ultrarrelativístico, durante o universo primordial até a época de transição radiaçãomatéria (densidade de energia da radiação igual à densidade de energia da matéria não relativística), como também nos estágios finais do processo de colapso gravitacional. Entretanto o fluido material dominante após a época da transição radiação-matéria será a matéria escura. Com isto, a equação de conservação da energia (2.33) para a mistura do fluido material e o vácuo dinâmico é:

$$
\dot{\rho}_{\Lambda}+\dot{\rho}+3 \mathcal{H} \rho(1+\omega)=0 .
$$

No caso do modelo $\Lambda \mathrm{CDM}$, onde $\dot{\rho}_{\Lambda}=0$, esta lei se reduz à conservação da energia associado unicamente ao fluido material dominante [Weinberg, 1972]

$$
\dot{\rho}+3 \mathcal{H} \rho(1+\omega)=0,
$$

que também é válida para cada fluido $x$ que interage apenas gravitacionalmente com as outras $M-1$ componentes do modelo. Note que a equação acima é válida independentemente dos outros $M-1$ fluidos possuir ou não alguma interação não gravitacional, e sua solução é dada por:

$$
\rho_{x}=c_{x} a^{-3\left(1+\omega_{x}\right)}
$$

onde $c_{x}$ é uma constante de integração.

- No caso da radiação, cuja equação de estado é dada pela expressão $\mathcal{P}_{r}=\rho_{r} / 3$, temos que a conservação da energia (2.35) -válida para qualquer dependência temporal do fator de escala, ou seja, qualquer que seja o conteúdo energético do universo- pode ser reescrita como:

$$
\frac{\partial \rho_{r}}{\partial \eta}+4 \mathcal{H} \rho_{r}=a^{-4} \frac{\partial\left(\rho_{r} a^{4}\right)}{\partial \eta}=0
$$


Esta equação tem como solução $\rho_{r}=c_{r} a^{-4}, \operatorname{com} a=a(\eta)$. Conforme explicamos anteriormente, esta solução é válida para qualquer valor do fator de escala, em particular para $a=a_{0}$ (no presente), onde podemos comparar a densidade de energia da radiação com a densidade média do universo (próxima da chamada densidade crítica $\rho_{c r}$ definida como a densidade total para um universo FRW plano). Escrevendo a densidade de energia da radiação em função da densidade crítica hoje $\rho_{c r}^{0}$ ( o índice 0 equivale a avaliar a grandeza associada na época atual) temos

$$
\rho_{r}=\rho_{r}^{0}\left(\frac{a_{0}}{a}\right)^{4} \equiv \Omega_{r}^{0} \rho_{c r}^{0}\left(\frac{a_{0}}{a}\right)^{4},
$$

onde $\Omega_{i} \equiv \rho_{i} / \rho_{c r}$ é a fração da densidade de energia do fluido $i$ em relação à densidade crítica.

- Em geral a equação conservação da energia (2.35) pode ser reescrita como:

$$
a^{-3} \frac{\partial\left[\rho a^{3}\right]}{\partial \eta}=-3 \mathcal{H} \mathcal{P} \text {. }
$$

Para o caso da matéria escura não relativística (chamada de matéria escura fria), cuja equação de estado pode ser escrita como $\mathcal{P}_{m}=0$ (independe do tempo e da densidade de energia $\rho_{m}$ ), a equação de conservação da energia (2.39) se reduz a:

$$
a^{-3} \frac{\partial\left[\rho_{m} a^{3}\right]}{\partial \eta}=0
$$

com solução $\rho_{m}=c_{m} a^{-3}$. Novamente, esta solução é válida para qualquer que seja a dependência temporal do fator de escala, isto é, para qualquer que seja o fluido dominante no universo. Assim, podemos obter a constante de integração $c_{m}$ da comparação com a densidade crítica no presente:

$$
\rho_{m}=\rho_{m}^{0}\left(\frac{a_{0}}{a}\right)^{3} \equiv \Omega_{m}^{0} \rho_{c r}^{0}\left(\frac{a_{0}}{a}\right)^{3}
$$

- Outra componente relevante no universo recente é a energia escura, que no modelo padrão tem a equação de estado simples $\mathcal{P}_{\Lambda}=-\rho_{\Lambda}$, e é modelada como sendo não interagente. Esta pressão negativa seria a responsável pela expansão acelerada do universo observada recentemente [Riess et al., 1998; Perlmutter et al., 1998, 1999]. Esta energia escura é conhecida como a constante cosmológica, já que a equação de conservação da energia (2.35) implica que:

$$
\frac{\partial \rho_{\Lambda}}{\partial \eta}=0
$$

e tem a solução $\rho_{\Lambda}=$ cte, isto é, não depende de $a(\eta)$ (por isso o nome de "constante cosmológica"). Dos dados observacionais podemos obter a relação entre a densidade de energia da constante cosmológica e a densidade crítica no presente:

$$
\rho_{\Lambda}=\rho_{\Lambda}^{0}=\rho_{c r}^{0} \Omega_{\Lambda}^{0}
$$

Do conjunto de equações $(2.38,2.41,2.43)$ vemos que as densidades de energia da radiação, da matéria escura e da constante cosmológica evoluem respetivamente como $\rho_{r} \propto a^{-4}, \rho_{m} \propto a^{-3}$ e $\rho_{\Lambda} \propto c t e$, mesmo 


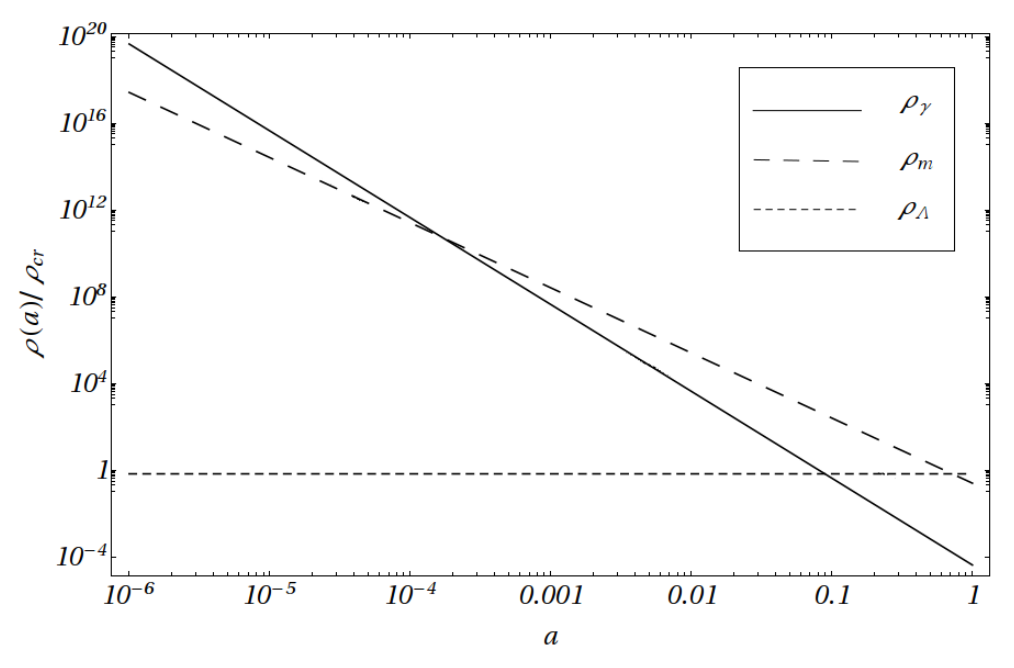

Figura 2.3: Evolução da densidade de energia da radiação, da matéria escura fria e da energia escura em função do fator de escala $a$ para o universo plano, homogêneo e isotrópico, normalizados em relação à densidade crítica hoje.

se todas elas estiverem presentes simultaneamente no universo, mas com a condição de que cada uma delas apresente apenas interações gravitacionais. Portanto, a densidade de energia da radiação decresce mais rapidamente que a densidade de energia da matéria com o aumento do fator de escala, enquanto a densidade da constante cosmológica permanece invariante em um universo descrito pela métrica FRW (2.16).

O modelo $\Lambda$ CDM tem como fluidos dominantes a radiação, a matéria escura modelada como sendo matéria escura não relativística (matéria escura fria) CDM e a energia escura modelada como uma constante $\Lambda$. Este modelo assume inicialmente (após o período inflacionário) a densidade de energia da radiação como sendo muito maior que a densidade de energia da matéria escura, e esta última por sua vez, muito maior que a densidade de energia escura, ou seja, a densidade de energia da radiação dominava a evolução do universo após a inflação.

Com estas condições iniciais $\left(\rho_{r} \gg \rho_{m} \gg \rho_{\Lambda}\right.$ no começo da era da radiação) e devido à relação entre as taxas de decrescimento das diferentes densidades de energia $\left(\dot{\rho}_{r} / \rho_{r}>\dot{\rho}_{m} / \rho_{m}>\dot{\rho}_{\Lambda} / \rho_{\Lambda}\right.$ para qualquer valor do tempo cosmológico em que cada componente evolua livremente) temos que o universo será dominado inicialmente pela radiação, depois pela matéria escura e mais tarde pela constante cosmológica.

Estes três períodos na evolução do universo são chamados, no contexto do modelo $\Lambda$ CDM, de período de domínio da radiação, período de domínio da matéria e período de domínio da constante cosmológica (se o modelo $\Lambda \mathrm{CDM}$ estiver certo, ainda estaríamos em um período de transição entre o domínio da matéria e o domínio da constante cosmológica). Chamamos de período de transição radiação-matéria a vizinhança ao redor do tempo de transição $\eta_{\text {eq }}$, caracterizado pela igualdade nas densidades de energia da radiação e da matéria. 
Voltando para o caso em que o universo é descrito pela métrica (2.16) e composto por $M$ fluidos, as componentes das equações de Einstein (2.26) com índices $\mu=v=0$ e $\mu=v=i$ são:

$$
8 \pi G a^{2} \rho_{\mathrm{T}}=3 \mathcal{H}^{2}+3 \kappa, \quad 8 \pi G a^{2} \mathcal{P}_{\mathrm{T}}=-2 \dot{\mathcal{H}}-\mathcal{H}^{2}-\kappa,
$$

enquanto que as componentes $\mu=i \neq v=j$ e $\{\mu, v\}=\{0, i\}$ são identicamente nulas devido às condições de perfeita homogeneidade e isotropia que estamos assumindo no presente capítulo. Com isto, temos que o escalar de Ricci (2.20) pode ser escrito em função do conteúdo material do sistema como:

$$
\mathcal{R}=8 \pi G\left(\rho_{T}-3 \mathcal{P}_{T}\right),
$$

esta expressão independe da curvatura espacial e é maior que cero para um sistema que contém energia escura, matéria escura fria, bários, neutrinos e radiação.

Vale a pena ressaltar que para um universo descrito pela métrica FRW (2.16) (que introduz um grau de liberdade correspondente ao fator de escala $a$ ) e composto por $M=N+I$ fluidos (que introduzem $2 M$ graus de liberdade correspondentes a $M$ densidades de energia e $M$ pressões), dos quais $N$ apresentam interação gravitacional e $I$ apresentam interações não gravitacionais com $P$ constantes de acoplamento diferentes, obtemos um sistema de $2 M+1$ equações diferenciais para a evolução dos $2 M+1$ graus de liberdade do sistema. Este sistema é composto por $M-P$ equações de conservação da energia ( $I-P$ equações da forma (2.33) para os conjuntos de fluidos com alguma interação entre eles além da gravitacional, e $N$ equações da forma (2.35) para os fluidos que interagem apenas de forma gravitacional); as $P$ equações que modelam as interações não gravitacionais entre os diferentes fluidos; as $M$ equações de estado dos fluidos; e a primeira das componentes (2.44) das equações de Einstein (a outra componente não nula pode se escrita como uma combinação linear das equações de conservação da energia e da primeira equação (2.44)).

Em geral, o sistema de $2 M$ equações diferenciais (composto pelo conjunto fechado de $2 M+1$ equações menos a equação de Einstein) pode ser resolvido em função do fator de escala $a$, e posteriormente podemos substituir as densidades de energia $\rho_{x}(a)$ na primeira equação (2.44) e integrá-la para encontrar o fator de escala em função do tempo:

$$
\eta=\int \frac{d a}{a \sqrt{8 \pi G a^{2} \rho_{T}(a) / 3-\kappa}} .
$$

No caso particular em que o universo é composto por radiação, matéria escura fria e uma constante cosmológica (todas não interagentes), as soluções das equações de conservação da energia (usando as respetivas equações de estado) estão dadas pelas expressões $(2.38,2.41,2.43)$, com o que a solução à primeira equação (2.44) pode ser expressa como:

$$
\eta=\int \frac{(1+z)^{2} d z}{H_{0} \sqrt{\Omega_{r}^{0}(1+z)^{4}+\Omega_{m}^{0}(1+z)^{3}+\Omega_{\mathcal{K}}^{0}(1+z)^{2}+\Omega_{\Lambda}^{0}}},
$$

onde definimos $\Omega_{\kappa}^{0} \equiv-\kappa / \mathcal{H}_{0}^{2}$ e $(1+z) \equiv 1 / a$.

Por outro lado, para um universo composto por $M$ fluidos, mas dominado pelo $x$-ésimo fluido que interagem apenas de forma gravitacional $\left(\rho_{T} \approx \rho_{x} \gg \rho_{y} \operatorname{com} y \in\{1,2, \ldots, x-1, x+1, \ldots, N-1, N\}\right)$, a evolução geral do universo pode ser aproximada pelas duas equações de Einstein correspondentes a um 
universo que contém o fluido dominante (considerando as demais componentes energéticas como fluidos de teste, que são afetados pela geometria global do universo, mas que não influenciam tal geometria):

$$
8 \pi G a^{2} \rho_{x}=3 \mathcal{H}^{2}, \quad 8 \pi G a^{2} \mathcal{P}_{x}=-2 \dot{\mathcal{H}}-\mathcal{H}^{2}
$$

Enquanto a equação de conservação da energia do fluido dominante é redundante.

Se conhecermos a equação de estado do fluido dominante $\mathcal{P}_{x}=\mathcal{P}_{x}(\rho)$ podemos juntar as equações (2.48) na seguinte equação:

$$
2 \dot{\mathcal{H}}+(1+3 \omega) \mathcal{H}^{2}=0,
$$

que pode ser integrada duas vezes no tempo conforme resultando em:

$$
\mathcal{H}(\eta)=\frac{2}{\eta(1+3 \omega)+2 c_{1}}, \quad a(\eta)=c_{2}\left\{\eta(1+3 \omega)+2 c_{1}\right\}^{2 /(1+3 \omega)}
$$

Com estas soluções podemos resolver as $2 M-1$ equações restantes que dão a evolução dos fluidos de teste.

No caso especial do modelo $\Lambda \mathrm{CDM}$, durante os períodos de domínio da radiação, da matéria escura fria e da constante cosmológica, a evolução global do universo pode ser descrita como mostraremos a seguir.

- Período de domínio da radiação.

Durante o período de domínio da radiação (quando densidade de energia da radiação é muito maior que as densidades de energia da matéria e da energia escura, ou seja, para $a \ll a_{e q}$ ) a dinâmica do universo pode ser aproximada pelas soluções à equação (2.49) dadas pelas expressões (2.50) $\operatorname{com} \omega=1 / 3$ :

$$
\mathcal{H}(\eta) \approx \frac{1}{\eta+c_{1 r}}, \quad a(\eta) \approx 2 c_{2 r}\left(\eta+c_{1 r}\right), \quad \text { ou } \quad a(t) \approx 2 \sqrt{c_{2 r}\left(t+c_{3 r}\right)}
$$

Usando as condições de fronteira, $\mathcal{H}\left(\eta_{e q}\right) \equiv \mathcal{H}_{e q}$ e $a\left(\eta_{e q}\right) \equiv a_{e q}$, podemos ajustar as constantes de integração e reescrever as soluções acima como:

$$
\mathcal{H}(a) \approx \mathcal{H}_{e q} \frac{a_{e q}}{a}, \quad a(\eta) \approx a_{e q}\left(1+\mathcal{H}_{e q}\left(\eta-\eta_{e q}\right)\right), \quad a(t) \approx a_{e q} \sqrt{1+H_{e q}\left(t-t_{e q}\right)}
$$

- Período de domínio da matéria escura fria.

Após o período de domínio da radiação no universo, para $a \gg a_{e q}$ no modelo $\Lambda \mathrm{CDM}$, surge o período de domínio da matéria (maioritariamente matéria escura fria). Durante este período, podemos aproximar a densidade total do universo (igual a $\rho_{c r}$ para um universo plano) como sendo a densidade de energia da matéria $\rho_{m}$, já que neste período as densidades de energia, da radiação e da constante cosmológica são desprezíveis se comparadas com a densidade de energia da matéria presente no universo. Com isto, a dinâmica do universo pode ser aproximada pelas expressões (2.50) com $\omega=0$ : 


$$
\mathcal{H}(\eta) \approx \frac{2}{\eta+2 c_{1 m}}, \quad a(\eta) \approx c_{2 m}\left(\eta+2 c_{1 m}\right)^{2}, \quad t(a) \approx \frac{a^{3 / 2}}{3 \sqrt{c_{2 m}}}+c_{3 m}
$$

Usando as condições de contorno na época de transição radiação-matéria, podemos encontrar as constantes de integração, com as que é possível reescrever as expressões que descrevem a dinâmica do universo durante o período de domínio da matéria:

$$
\begin{gathered}
\mathcal{H}(a) \approx \mathcal{H}_{e q} \sqrt{\frac{a_{e q}}{a}}, \quad a(\eta) \approx a_{e q}\left(1+\frac{\mathcal{H}_{e q}}{2}\left(\eta-\eta_{e q}\right)\right)^{2}, \\
t(a) \approx t_{e q}+\frac{2}{3 H_{e q}}\left(\left(\frac{a}{a_{e q}}\right)^{3 / 2}-1\right) .
\end{gathered}
$$

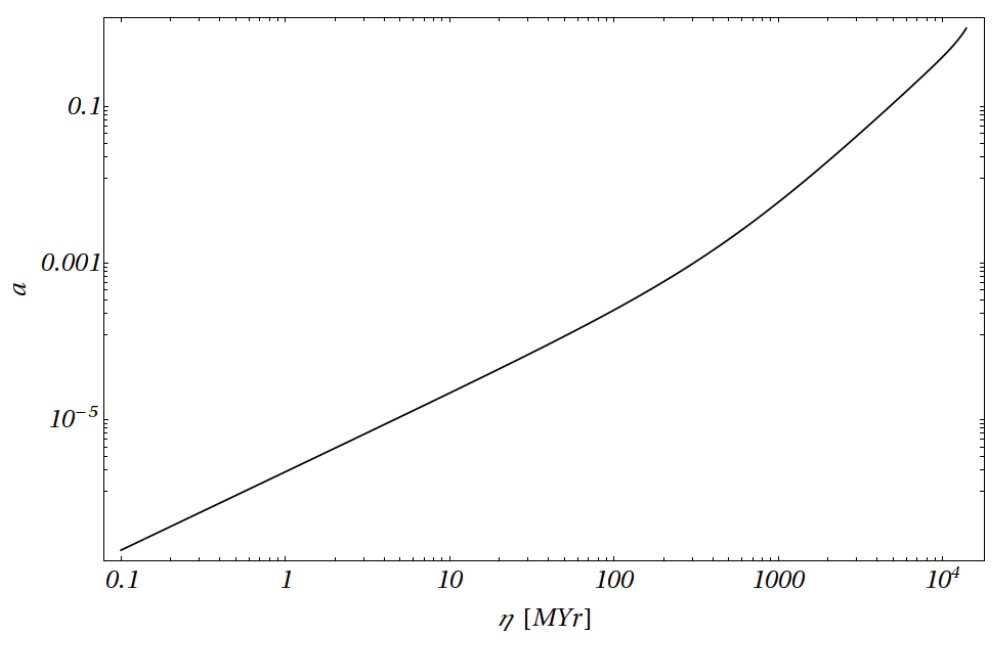

Figura 2.4: Evolução do fator de escala como função do tempo conforme para o modelo $\Lambda C D M$.

- Transição radiação - matéria

Para nosso conforto, as soluções da equação de Friedman (2.48) que nos dão a dependência temporal do fator de escala $a(\eta)$ para o período de domínio da radiação (2.52) e para o período de domínio da matéria (2.53), são compatíveis. Isto é, a solução da equação de Friedmann (2.48) para $\rho \approx \rho_{r}+\rho_{m} \approx \rho_{0}$, que nos dá a dependência temporal do fator de escala para o período em que a densidade da energia escura é desprezível, se comparadas com a soma das densidades da radiação e da matéria, é:

$$
a(\eta) \approx \frac{2 \pi G \rho_{0}}{3} \Omega_{m} \eta^{2}+\sqrt{\frac{8 \pi G \rho_{0}}{3} \Omega_{r} \eta} .
$$

- Período de domínio da constante cosmológica.

Após os períodos de domínio do universo por parte da densidade de energia da radiação e da matéria, e devido a que densidade de energia escura neste modelo permanece constante ao longo do tempo, teremos um período de domínio da energia escura (constante cosmológica no modelo $\Lambda \mathrm{CDM})$. Este período é caracterizado por um crescimento acelerado do fator de escala, causado pela pressão negativa da constante cosmológica. Vamos aproximar a densidade da energia total 
do universo pela densidade da energia escura $\rho \approx \rho_{\Lambda}$, desta forma a dinâmica do universo pode ser aproximada pelas expressões (2.50) $\operatorname{com} \omega=-1$ :

$$
\mathcal{H}(\eta) \approx \frac{1}{c_{1 \Lambda}-\eta}, \quad a(\eta) \approx \frac{c_{2 \Lambda}}{2 c_{1 \Lambda}-2 \eta}, \quad a(t) \approx e^{\left(c_{3 \Lambda}-t\right) 2 / c_{2 \Lambda}}
$$

e ajustadas condições de fronteira hoje $a\left(\eta_{0}\right) \equiv a_{0}$ e $H\left(a_{0}\right) \equiv H_{0}$, podemos reescrever estes resultados como:

$$
H \approx H_{0}, \quad a(\eta) \approx \frac{a_{0}}{1+\mathcal{H}_{0}\left(\eta-\eta_{0}\right)}, \quad a(t) \approx a_{0} e^{H_{0}\left(t-t_{0}\right)}
$$



PERTURBAÇÕES LINEARES NO UNIVERSO FRW PLANO

No capítulo anterior introduzimos as equações que descrevem a evolução de um universo com curvatura espacial constante, perfeitamente homogêneo e isotrópico espacialmente, em expansão, e composto por um conjunto de fluidos que podem em geral apresentar interações além da interação gravitacional.

Como vimos no capítulo anterior, as equações que descrevem a dinâmica do universo na aproximação de homogeneidade e isotropia são: as equações de estado $\mathcal{P}_{x}=\mathcal{P}_{x}\left(\rho_{x}\right)$ para cada um dos fluidos presentes no universo; as equações de conservação da energia tanto dos diferentes conjuntos de fluidos que possuem alguma interação em comum além da gravitacional, como as equações individuais para a conservação da energia dos fluidos que apresentam apenas interação gravitacional (trata-se apenas das componentes temporais das equações de conservação covariante do tensor momento energia, já que as componentes espaciais são identicamente nulas se assumirmos isotropia e homogeneidade); as equações que descrevem as interações não gravitacionais entres os diferentes fluidos (não especificadas no capítulo anterior); e as componentes da equação de Einstein (duas são nulas se assumirmos isotropia e homogeneidade, e uma é redundante se conhecermos as equações de estado e de conservação para cada fluido presente no universo).

No caso de um universo espacialmente plano e composto por radiação, matéria escura fria e constante cosmológica, cada uma destas interagindo apenas de forma gravitacional, se conhecermos as equações de estado para os três fluidos teremos um sistema de 4 equações (a componente temporal da equação de conservação do tensor momento energia para cada um dos três fluidos, e uma componente da equação de Einstein) e 4 incógnitas (a densidade de energia para cada fluido em função do fator de escala, e o fator de escala em função do tempo). Neste contexto, as três equações de conservação da energia podem ser resolvidas de forma exata independentemente do período de evolução que o universo esteja atravessando, enquanto que a equação de Einstein pode ser resolvida de forma analítica durante três épocas desconexas: a épocas de domínio da radiação, da matéria, e da constante cosmológica (ver sessão $2.2)$.

As observações cosmológicas [Mukhanov, 2005] mostram que o universo em grandes escalas (da ordem de 100 Mpc ou maiores) é bastante homogêneo e isotrópico, mas apresenta pequenas flutuações na sua homogeneidade e isotropia, sendo assim, podemos modelá-las usando teoria de perturbações lineares. Nesta seção introduziremos perturbações escalares tanto no tensor métrico como também no tensor momento energia da radiação e da matéria para modelar o nosso universo em grandes escalas. Para estudar a evolução destas perturbações assumiremos que as equações até ordem zero nas perturbações (sem perturbações) são válidas e apresentam as soluções do capítulo anterior; posteriormente, a partir 
da equação de conservação do tensor momento-energia para a radiação e a matéria, e das componentes da equação de Einstein, obteremos as equações de evolução das perturbações até primeira ordem.

Veremos também que as perturbações métricas podem ser escritas em diferentes calibres. Adicionalmente, mostraremos que invariantes de calibre podem ser construídos, e que as equações de Einstein podem ser escritas numa forma explicitamente invariante de calibre. Estes dois fatos nos permitem trabalhar em um calibre que simplifique as equações de movimento de um casso particular arbitrário, e mudar de calibre quando necessário, fazendo a junção entre diferentes calibres com a ajuda dos diferentes invariantes de calibre. Este procedimento será de particular utilidade no capítulo 4, na hora de calcular o espectro de potências das perturbações lineares no universo recente, a partir das perturbações geradas durante o período inflacionário.

\subsection{PERTURBAÇÕES LINEARES NO TENSOR MÉTRICO}

Graças à formulação tensorial da gravitação de Einstein, em que está baseado o modelo cosmológico padrão, é possível introduzir perturbações escalares, vetoriais e tensoriais no tensor métrico. A métrica de Friedmann-Robertson-Walker (em coordenadas Cartesianas) para um universo espacialmente plano, em expansão, perturbada em relação às condições de homogeneidade e isotropia, pode ser parametrizada na forma mais geral como:

$$
\begin{aligned}
& g_{00}\left(x^{\mu}\right)=-a^{2}(\tau)\left\{1+2 \psi\left(x^{\mu}\right)\right\}, \\
& g_{0 i}\left(x^{\mu}\right)=a^{2}(\tau) \omega_{i}\left(x^{\mu}\right), \\
& g_{i j}\left(x^{\mu}\right)=a^{2}(\tau)\left\{\left[1-2 \phi\left(x^{\mu}\right)\right] \delta_{i j}+\hbar_{i j}\left(x^{\mu}\right)\right\},
\end{aligned}
$$

sendo $g_{\mu \nu}=\bar{g}_{\mu \nu}+h_{\mu v}$, onde $\bar{g}_{\mu \nu}$ é o tensor métrico não perturbado e $h_{\mu \nu}$ é a perturbação da métrica escrita como um tensor, e depende do ponto $x^{\mu}$ onde é avaliada. Como o tensor $h_{\mu \nu}$ é simétrico e de ordem 2, em quatro dimensões terá no máximo 10 componentes independentes. Estas 10 componente podem ser escritas como dois campos escalares (tensores de ordem zero) $\phi$ e $\psi$, correspondendo a 2 graus de liberdade, um tri-vetor $\omega_{i}$ (tensor de grau um em 3 dimensões) com 3 graus de liberdade, e um tensor de ordem dois $h_{i j}$ (em três dimensões) simétrico, que possui 5 graus de liberdade. O traço do tensor perturbação da métrica é dado pelos campos escalares $\psi$ e $\phi$. No total, os campos $\phi, \psi, \omega_{i}$ e $\hbar_{i j}$ que compõem a perturbação ao tensor métrico somam 10 de graus de liberdade.

Alternativamente podemos decompor os campos $\omega_{i}$ e $\boldsymbol{h}_{i j}$ em função de tensores irredutíveis como sendo:

$$
\omega=\omega^{\|}+\omega_{\perp}, \quad \text { onde } \quad \nabla \times \omega^{\|}=0, \quad \text { e } \quad \nabla \cdot \omega^{\perp}=0 .
$$

Assim, o campo $\boldsymbol{\omega}^{\|}$pode ser reescrito como sendo o gradiente de um campo escalar B:

$$
\omega_{i}^{\|} \equiv \partial_{i} B
$$

portanto terá apenas 1 grau de liberdade. Enquanto que a restrição $\nabla \cdot \omega^{\perp}=0$ deixa o campo vetorial $\omega^{\perp}$ com com 2 graus de liberdade (e não pode ser escrito em função de um campo escalar, portanto é um vetor irredutível). 
Igualmente, o tensor simétrico sem traço $h_{i j}$ (lembrando que os índices latinos correm de 1 até 3 , enquanto que os índices gregos correm de 0 até 3 ) pode ser escrito como:

$$
h_{i j}=h_{i j}^{\|}+h_{i j}^{\perp}+h_{i j}^{T}, \quad \text { com } \quad h \equiv h_{i}^{i}=0,
$$

e onde as componentes longitudinal $h_{i j}^{\|}$, solenoidal $h_{i j}^{\perp}$ e a componente transversal sem traço $h_{i j}^{T}$ estão definidas como tendo as propriedades:

$$
\epsilon^{i j k} \partial_{j} \partial^{l} \kappa_{l k}^{\|}=0, \quad \partial^{i} \partial^{j} \kappa_{i j}^{\perp}=0, \quad \partial^{i} \kappa_{i j}^{T}=0 .
$$

Com isto, a componente longitudinal do tensor $\hbar$ pode ser reescrita em função de um campo escalar $\mu$ (1 grau de liberdade) e a componente solenoidal pode ser escrita em função de um campo vetorial transverso $A_{i}\left(\partial^{i} A_{i}=0\right.$ com 2 graus de liberdade) da seguinte forma:

$$
\kappa_{i j}^{\|}=\left(\partial_{i} \partial_{j}-\frac{1}{3} \delta_{i j} \nabla^{2}\right) \mu, \quad \kappa_{i j}^{\perp}=2 \partial_{(i} A_{j)} \equiv \partial_{i} A_{j}+\partial_{j} A_{i}, \quad \partial_{i} A_{i}=0 .
$$

A componente $\boldsymbol{h}_{i j}^{T}$ não pode ser decomposta, ficando explicitamente escrita como um tensor espacial simétrico $3 \times 3$ sem traço e com 2 graus de liberdade. Estas 6 componentes irredutíveis são transformadas individualmente em quantidades do mesmo tipo sobre rotações espaciais, e constituem o equivalente à representação irredutível deste grupo de simetrias [Carroll, 2004].

Resumindo, a decomposição da perturbação do tensor métrico em tensores irredutíveis esta definida por quatro campos escalares $\psi, \phi, \mu$ e $B$ com 1 grau de liberdade cada, dois campos vetoriais transversos $A_{i}$ e $\omega_{i}^{\perp}$ cada um com 2 graus de liberdade, e finalmente por um tensor $3 \times 3$ transverso sem traço $h_{i j}^{T}$, que possui 2 graus de liberdade. Em termos de estes tensores irredutíveis, o tensor momento energia se escreve como:

$$
\begin{aligned}
& g_{00}\left(x^{\mu}\right)=-a^{2}(\tau)\left[1+2 \psi\left(x^{\mu}\right)\right], \\
& g_{0 i}\left(x^{\mu}\right)=a^{2}(\tau)\left[\partial_{i} B\left(x^{\mu}\right)+\omega_{i}^{\perp}\left(x^{\mu}\right)\right], \\
& g_{i j}\left(x^{\mu}\right)=a^{2}(\tau)\left\{\left[1-2 \phi\left(x^{\mu}\right)\right] \delta_{i j}+\partial_{i} A_{j}\left(x^{\mu}\right)+\partial_{j} A_{i}\left(x^{\mu}\right)+\left[\partial_{i} \partial_{j}-\delta_{i j} \nabla^{2} / 3\right] \mu\left(x^{\mu}\right)+\kappa_{i j}^{T}\left(x^{\mu}\right)\right\},
\end{aligned}
$$

e com $\partial^{i} \omega_{i}^{\perp}=\partial^{i} A_{i}=\partial^{i} f_{i j}^{T}=0$.

Estas três perturbações irredutíveis (escalares, vetores transversos e tensores transversos sem traço) evoluem independentemente no regime linear segundo o teorema de decomposição [Peebles, 1980; Ma and Bertschinger, 1995; Dodelson, 2003; Mukhanov, 2005], e cada uma é responsável por um efeito diferente: formação de estruturas (perturbações escalares), gravito-magnetismo (perturbações vetoriais) e ondas gravitacionais (perturbações tensoriais).

\subsubsection{Liberdade de calibre}

Dos 10 graus de liberdade do tensor métrico $g_{\mu \nu}$, apenas 6 são independentes e podem representar graus de liberdade físicos, já que os observáveis físicos devem ser invariantes por transformações de coordenadas, o que introduz 4 restrições. Os 6 graus de liberdade físicos correspondem a 2 graus de liberdade para cada tipo de perturbação (escalar, vetorial e tensorial). Assim, os 10 graus de liberdade 
do tensor $g_{\mu v}$ podem ser expressos como função de 6 componentes físicas independentes, o que nos dá a liberdade de expressar o tensor $g_{\mu \nu}$ de formas diferentes sem mudar os observáveis físicos. Isto é chamado de liberdade de calibre.

Esta liberdade pode ser vista formalmente [Carroll, 2004] se pensamos em dois espaço-tempos difeomorfos, que chamaremos de $M_{p}$ (espaço-tempo de prova com a métrica $\tilde{g}_{\mu v} \equiv \bar{g}_{\mu v}+\tilde{f}_{\mu v}$ ) e $M_{f}$ (espaço-tempo físico com a métrica física $\left.g_{\mu v} \equiv \bar{g}_{\mu v}+f_{\mu v}\right)$, relacionados pelo difeomorfismo $\phi: M_{p} \rightarrow M_{f}$. As duas métricas estão relacionadas pelo mapa $\phi^{*}$ induzido por $\phi$, e definido da seguinte forma:

$$
\bar{g}_{\mu v}+\tilde{f}_{\mu v}=\left(\phi^{*} g\right)_{\mu v} .
$$

Agora, a partir das curvas integrais de um campo vetorial arbitrário $\xi^{\mu}$ podemos definir um difeomorfismo $\psi_{\epsilon}: M_{p} \rightarrow M_{p}$, parametrizado por $\epsilon(\epsilon \ll 1$ para que a perturbação da métrica ao redor da métrica $\bar{g}_{\mu \nu}$ seja ainda linear). Sobre a ação do difeomorfismo definido como a composição $\left(\phi \circ \psi_{\epsilon}\right)$ a perturbação métrica se transforma como:

$$
f_{\mu v}=\left[\left(\phi \circ \psi_{\epsilon}\right)^{*} g\right]_{\mu v}-\bar{g}_{\mu v}=\left[\psi_{\epsilon}^{*}\left(\phi^{*} g\right)\right]_{\mu v}-\bar{g}_{\mu v}=\psi_{\epsilon}^{*}\left(\tilde{f}_{\mu v}\right)+\psi_{\epsilon}^{*}\left(\bar{g}_{\mu v}\right)-\bar{g}_{\mu v},
$$

e graças a que $\epsilon \ll 1$ podemos aproximar $\psi_{\epsilon}^{*}\left(\tilde{h}_{\mu v}\right)=\tilde{h}_{\mu v}$, já que suas correções são da ordem $\mathcal{O}\left(\epsilon^{2}\right)$ e podemos desconsiderar-lhas na aproximação linear. Por outro lado, usando a definição da derivada de Lie

$$
\mathcal{L}_{\xi} \bar{g}_{\mu \nu}=\lim _{\epsilon \rightarrow 0} \frac{\psi_{\epsilon}^{*}\left(\bar{g}_{\mu \nu}\right)-\bar{g}_{\mu v}}{\epsilon},
$$

que atuando sobre um tensor arbitrário $T$ do tipo $(m, n)$ produz a expressão

$$
\begin{aligned}
\mathcal{L}_{\xi} T^{v_{1} \cdots v_{n}}{ }_{\mu_{1} \cdots \mu_{m}}= & \xi^{\lambda} \partial_{\lambda} T^{v_{1} \cdots v_{n}}{ }_{\mu_{1} \cdots \mu_{m}}+ \\
& -T^{\lambda v_{2} \cdots v_{n}}{ }_{\mu_{1} \cdots \mu_{m}} \partial_{\lambda} \xi^{v_{1}}-\cdots-T^{v_{1} \cdots v_{n-1} \lambda}{ }_{\mu_{1} \cdots \mu_{m}} \partial_{\lambda} \xi^{v_{n}}+ \\
& +T^{v_{1} \cdots v_{n}}{ }_{\lambda \mu_{2} \cdots \mu_{m}} \partial_{\mu_{1}} \xi^{\lambda}+\cdots+T^{v_{1} \cdots v_{n}}{ }_{\mu_{1} \cdots \mu_{m-1} \lambda} \partial_{\mu_{m}} \xi^{\lambda},
\end{aligned}
$$

(em particular, para qualquer dois campos vetoriais arbitrários $\xi$ e $\zeta$ temos que $\mathcal{L}_{\xi} \zeta=\xi \zeta-\zeta \xi \equiv[\xi, \zeta]$ ), temos que sua aplicação ao tensor métrico $g$ gera a expressão simples

$$
\mathcal{L}_{\xi} g_{\mu \nu}=\xi^{\lambda} \partial_{\lambda} g_{\mu \nu}+g_{\lambda \nu} \partial_{\mu} \xi^{\lambda}+g_{\mu \lambda} \partial_{\nu} \xi^{\lambda}
$$

com isto, obtemos que a variação na perturbação métrica (para $\epsilon_{\mu v} \ll 1$ ) gerada pela aplicação do difeomorfismo $\phi \circ \psi_{\epsilon}$ pode ser escrita como

$$
f_{\mu v}=\tilde{\epsilon}_{\mu v}+\xi^{\lambda} \partial_{\lambda} \bar{g}_{\mu v}+\epsilon \bar{g}_{\lambda v} \partial_{\mu} \xi^{\lambda}+\epsilon \bar{g}_{\mu \lambda} \partial_{\nu} \xi^{\lambda}
$$

até primeira ordem em $\epsilon$ e $f_{\mu v}$, onde usamos $\psi_{\epsilon}^{*}\left(\tilde{h}_{\mu v}\right) \approx \tilde{f}_{\mu v}$ até primeira ordem. Agora fica evidente (equação (3.13)) que a decomposição de uma métrica como sendo a métrica $\bar{g}_{\mu v}$ mais uma perturbação não é única, ela depende do tipo de coordenadas usadas, já que um difeomorfismo pode ser visto como uma transformação dinâmica de coordenadas. Similarmente acontece para a métrica FRW plana, ou para métricas mais gerais [Carroll, 2004], nos permitindo trabalhar com diferentes representações do mesmo sistema físico, já que na teoria de perturbações lineares as componentes do tensor de Riemann não dependem do calibre [Carroll, 2004]: $R_{\mu v \sigma \delta}=\tilde{R}_{\mu \nu \sigma \delta}$ até primeira ordem nas perturbações. 
Absorvendo o parâmetro infinitesimal $\epsilon$ dentro do próprio $\xi^{\mu}\left(\epsilon \xi^{\mu} \rightarrow \xi^{\mu} \ll 1\right)$ podemos reescrever a expressão (3.13) como:

$$
\tilde{g}_{\mu \nu}-g_{\mu v}=\tilde{\hbar}_{\mu v}-f_{\mu v}=-\xi^{\lambda} \partial_{\lambda} \bar{g}_{\mu \nu}-\left(\partial_{\mu} \xi^{\lambda}\right) \bar{g}_{\lambda v}-\left(\partial_{\nu} \xi^{\lambda}\right) \bar{g}_{\mu \lambda}
$$

Esta expressão define a variação das perturbações métricas gerada pela a mudança entre os sistemas de coordenadas $x$ e $\tilde{x}$ relacionados pelo difeomorfismo

$$
x^{\mu} \rightarrow \tilde{x}^{\mu}=x^{\mu}+\xi^{\mu}(x),
$$

onde $\xi^{\mu}$ é um campo vetorial arbitrário, que por sua vez pode ser decomposto como

$$
\xi^{\mu}=\left(\xi^{0}, \partial^{i} \xi+\xi_{t r}^{i}\right)
$$

onde $\xi^{0}$ e $\xi$ são funções escalares, sendo $\xi_{, i}$ a componente longitudinal de $\xi_{i}$ (isto é $\nabla \times \xi=0$ ), enquanto que $\xi_{t r}^{i}$ é a parte transversal de $\xi^{i}$ (ou seja, $\partial_{i} \xi_{t r}^{i}=0$ ), e são todos da ordem das perturbações métricas.

Da componente 00 da equação (3.14) se obtém diretamente a variação da perturbação $\psi$ sobre a transformação de coordenadas (3.15):

$$
\tilde{\psi}=\psi-\mathcal{H} \xi^{0}-\dot{\zeta}^{0}
$$

da componente $0 i$ obtemos a relação:

$$
\tilde{\omega}_{i}=\omega_{i}-\dot{\xi}_{t r}^{i}-\dot{\xi}_{, i}+\tilde{\zeta}_{, i}^{0}
$$

que pode ser decomposta em sua parte escalar, e em sua parte vetorial irredutível:

$$
\begin{aligned}
& \tilde{\omega}_{i}^{\|}=\omega_{i}^{\|}-\dot{\xi}_{, i}+\xi_{, i}^{0}, \\
& \tilde{\omega}_{i}^{\perp}=\omega_{i}^{\perp}-\dot{\xi}_{t r}^{i} .
\end{aligned}
$$

A componente $i j$ da equação (3.14) produz a seguinte relação:

$$
-2(\tilde{\phi}-\phi) \delta_{i j}+\tilde{\digamma}_{i j}-\kappa_{i j}=-2 \mathcal{H} \xi^{0} \delta_{i j}-\partial_{i} \xi_{t r}^{j}-\partial_{j} \xi_{t r}^{i}-2 \partial_{i} \partial_{j} \xi
$$

Multiplicando esta última equação por $\delta_{i j}$ obtemos que a variação do campo $\phi$ esta dada por

$$
\tilde{\phi}=\phi+\mathcal{H} \xi^{0}+\frac{1}{3} \nabla^{2} \xi
$$

onde usamos o fato de que $\kappa_{i i}=0$ e $\partial_{i} \xi_{t r}^{i}=0$. Se agora multiplicamos a última expressão por $2 \delta_{i j}$ e a somamos com a equação (3.19) obtemos

$$
\tilde{\kappa}_{i j}-\kappa_{i j}=-\partial_{i} \xi_{t r}^{j}-\partial_{j} \xi_{t r}^{i}-2\left(\partial_{i} \partial_{j}-\frac{1}{3} \nabla^{2}\right) \xi
$$


cujas componentes escalar, vetorial e tensorial irredutível estão dadas por:

$$
\begin{aligned}
\tilde{\kappa}_{i j}^{\|}-\kappa_{i j}^{\|} & =-2\left(\partial_{i} \partial_{j}-\frac{1}{3} \nabla^{2}\right) \xi, \\
\tilde{h}_{i j}^{\perp}-\kappa_{i j}^{\perp} & =-\partial_{i} \xi_{t r}^{j}-\partial_{j} \xi_{t r}^{i}, \\
\tilde{\kappa}_{i j}^{T}-\kappa_{i j}^{T} & =0 .
\end{aligned}
$$

Usando as relações $(3.3,3.6)$ podemos re-escrever os resultados descritos pelas expressões $(3.17,3.18,3.21)$ como sendo [Ma and Bertschinger, 1995]:

$$
\begin{aligned}
\tilde{\phi} & =\phi+\mathcal{H} \xi^{0}+\frac{1}{3} \nabla^{2} \xi, \\
\tilde{\psi} & =\psi-\mathcal{H} \xi^{0}-\dot{\xi}^{0}, \\
\tilde{B} & =B+\xi^{0}-\dot{\xi}^{\prime}, \\
\tilde{\mu} & =\mu-2 \xi, \\
\tilde{\omega}_{i}^{\perp} & =\omega_{i}^{\perp}-\dot{\xi}_{t r}^{i}, \\
\tilde{A}^{i} & =A^{i}-\xi_{t r}^{i}, \\
\tilde{\kappa}_{i j}^{T} & =\kappa_{i j}^{T} .
\end{aligned}
$$

Estas expressões descrevem a variação das perturbações escalares $\{\phi, \psi, B, \mu\}$, das perturbações vetoriais irredutíveis $\left\{\omega_{i}^{\perp}, A_{i}\right\}$ e das perturbação tensorial irredutível $\kappa_{i j}$ sobre o difeomorfismo associado à mudança de coordenadas descrita por (3.15), e com eles podem-se construir os seguintes invariantes de calibre independentes entre sé:

$$
\begin{aligned}
\Phi & =\tilde{\Phi} \equiv \phi-\mathcal{H}\left(B-\frac{\dot{\mu}}{2}\right)+\frac{1}{6} \nabla^{2} \mu, \\
\Psi & =\tilde{\Psi} \equiv \psi+a^{-1} \partial_{0}\left[a\left(B-\frac{\dot{\mu}}{2}\right)\right], \\
W_{i} & =\tilde{W}_{i} \equiv \omega_{i}^{\perp}-\dot{A}_{i}, \\
\tilde{h}_{i j} & =\kappa_{i j} .
\end{aligned}
$$

Os primeiros dois invariantes são conhecidos como potenciais de Bardeen [Bardeen, 1980], e representam os únicos invariantes de calibre que envolvem apenas as perturbações escalares da métrica; o terceiro é o único invariante de calibre vetorial que pode ser construído usando somente as perturbações métricas; enquanto que a perturbação tensorial da métrica (última linha) é invariante de calibre.

\subsection{PERTURBAÇÕES ESCALARES}

Levando em conta somente perturbações escalares, a métrica (3.1) toma a forma:

$$
\begin{aligned}
& g_{00}=-a^{2}(\tau)\left\{1+2 \psi\left(x^{\mu}\right)\right\}, \\
& g_{0 i}=a^{2}(\tau) \omega_{i}^{\|}\left(x^{\mu}\right), \\
& g_{i j}=a^{2}(\tau)\left\{\left[1-2 \phi\left(x^{\mu}\right)\right] \delta_{i j}+\kappa_{i j}^{\|}\left(x^{\mu}\right)\right\},
\end{aligned}
$$


onde $\omega_{i}^{\|}$(3.3) é $\boldsymbol{\hbar}_{i j}^{\|}$(3.6). Neste caso os símbolos de Christofel não nulos são:

$$
\begin{aligned}
& \Gamma_{00}^{0}=\mathcal{H}+\dot{\psi}, \\
& \Gamma_{0 i}^{0}=\psi_{, i}+\mathcal{H} B_{, i}, \\
& \Gamma_{00}^{i}=\psi_{, i}+\mathcal{H} B_{, i}+\dot{B}_{, i}, \\
& \Gamma_{j 0}^{i}=\delta_{i j}(\mathcal{H}-\dot{\phi})+\frac{\dot{h}_{i j}^{\|}}{2}, \\
& \Gamma_{i j}^{0}=\delta_{i j}(\mathcal{H}(1-2 \psi-2 \phi)-\dot{\phi})-B_{, i j}+\mathcal{H} \hat{h}_{i j}^{\|}+\frac{\dot{h}_{i j}^{\|}}{2}, \\
& \Gamma_{j k}^{i}=-\left(\phi_{, j} \delta_{k}^{i}+\phi_{, k} \delta_{j}^{i}-\phi^{, i} \delta_{j k}\right)-\mathcal{H} B_{, i} \delta_{j k}+\left(\frac{\hat{f}_{i j, k}^{\|}}{2}+\frac{f_{k i, j}^{\|}}{2}-\frac{f_{j k, i}^{\|}}{2}\right),
\end{aligned}
$$

similarmente, as componentes do tensor de Ricci são:

$$
\begin{gathered}
R_{00}=-3 \dot{\mathcal{H}}+3 \ddot{\phi}+3 \mathcal{H}(\dot{\psi}+\dot{\phi})-k^{2}(\psi+\mathcal{H} B+\dot{B}), \\
R_{0 i}=2 \partial_{i}(\dot{\phi}+\mathcal{H} \psi)+\left(\dot{\mathcal{H}}+2 \mathcal{H}^{2}\right) \partial_{i} B+\frac{\dot{h}_{i k, k}^{\|}}{2} \\
R_{i j}=\delta_{i j}\left(\left(\dot{\mathcal{H}}+2 \mathcal{H}^{2}\right)(1-2 \phi-2 \psi)-\ddot{\phi}-\mathcal{H}(\dot{\psi}+5 \dot{\phi})+\nabla^{2}(\phi+\mathcal{H} B)\right)+ \\
+\partial_{i} \partial_{j}(\phi-\psi-2 \mathcal{H} B-\dot{B})+ \\
+\dot{\mathcal{H}} h_{i j}^{\|}+\mathcal{H} \dot{h}_{i j}^{\|}+2 \mathcal{H}^{2} h_{i j}^{\|}+\left(\frac{h_{j k, i k}^{\|}}{2}+\frac{h_{i k, j k}^{\|}}{2}-\frac{h_{i j, k k}^{\|}}{2}+\frac{\ddot{h}_{i j}^{\|}}{2}\right),
\end{gathered}
$$

e as componentes do tensor de Einstein são:

$$
\begin{gathered}
a^{2} G_{0}^{0}=-3 \mathcal{H}^{2}+2\left(3 \mathcal{H}(\dot{\phi}+\mathcal{H} \psi)-\nabla^{2}(\phi-\mathcal{H} B)-\frac{\hat{f}_{k l, k l}^{\|}}{4}\right), \\
a^{2} G_{i}^{0}=-2 \partial_{i}(\dot{\phi}+\mathcal{H} \psi)-\frac{\dot{h}_{i k, k}^{\|}}{2} \\
a^{2} G_{j}^{i}=\delta_{i j}\left(-\left(2 \dot{\mathcal{H}}+\mathcal{H}^{2}\right)(1-2 \psi)+2 \mathcal{H}(\dot{\psi}+2 \phi)+2 \ddot{\phi}+\nabla^{2}(\psi-\phi+\dot{B}+2 \mathcal{H} B)-\frac{\hat{f}_{k l, k l}^{\|}}{2}\right)+ \\
-\partial_{i} \partial_{j}(\psi-\phi+\dot{B}-2 \mathcal{H} B)+\mathcal{H} \dot{h}_{i j}^{\|}+\left(\frac{h_{j k, i k}^{\|}}{2}+\frac{f_{i k, j k}^{\|}}{2}-\frac{f_{i j, k k}^{\|}}{2}+\frac{\ddot{h}_{i j}^{\|}}{2}\right) .
\end{gathered}
$$




\subsubsection{Perturbações no tensor momento energia}

Partiremos do tensor momento energia para $M$ fluidos (2.30):

$$
T^{\mu \nu}=\sum_{X=1}^{M}\left(\mathcal{P}_{X} g^{\mu \nu}-\left(\rho_{X}+\mathcal{P}_{X}\right) U_{X}^{\mu} U_{X}^{v}\right)
$$

onde $U_{X}$ é a 4-velocidade para cada fluido (lembrando que em coordenadas comoveis as suas componentes espaciais são zero para um gás não perturbado), enquanto que $\rho_{X}$ e $\mathcal{P}_{X}$ são suas densidades de energia e pressão medidas por um observador comóvel com a métrica $g^{\mu v}$. Se os fluidos indexados pela variável discreta $X$ possuírem uma velocidade peculiar pequena, $\left|v_{X}^{i}\left(x^{\mu}\right)\right|=\left|d x_{X}^{i} / d \eta\right| \ll 1$, mas diferente de zero e que em geral depende do ponto de avaliação, a consideraremos como uma perturbação em relação ao fluxo de Hubble. Uma perturbação deste tipo na velocidade dos fluidos gera uma diferença na sua pressão $\delta \mathcal{P}_{X}\left(x^{\mu}\right)$ e na densidade de energia $\delta \rho_{X}\left(x^{\mu}\right)$ se comparadas com a pressão e densidade de energia médias, $\overline{\mathcal{P}}_{X}$ e $\bar{\rho}_{X}$. Desta forma teremos que em geral $\rho_{X}\left(x^{\mu}\right)=\bar{\rho}_{X}+\delta \rho_{X}\left(x^{\mu}\right)$ e $\mathcal{P}_{X}\left(x^{\mu}\right)=\overline{\mathcal{P}}_{X}+\delta \mathcal{P}_{X}\left(x^{\mu}\right)$, onde $\left|\delta \rho_{X}\left(x^{\mu}\right)\right| \ll \bar{\rho}_{X}$ e $\left|\delta \mathcal{P}_{X}\left(x^{\mu}\right)\right| \ll \overline{\mathcal{P}}_{X}$, assim como $v_{X}^{i}\left(x^{\mu}\right) \ll 1$, dependem das coordenadas espaciais $x^{\mu}$ para cada fluido $X$. Em geral, estas perturbações podem acabar afetando a isotropia do tensor momento energia que, uma vez perturbado, pode ser parametrizado como:

$$
\begin{aligned}
& T_{0}^{0}\left(x^{\mu}\right)=-\rho_{T}\left(x^{\mu}\right) \equiv-\sum_{X}\left[\bar{\rho}_{X}+\delta \rho_{X}\left(x^{\mu}\right)\right], \\
& T_{0}^{i}\left(x^{\mu}\right)=\sum_{X}\left[\bar{\rho}_{X}+\overline{\mathcal{P}}_{X}\left(x^{\mu}\right)\right] \times v_{X}^{i}\left(x^{\mu}\right), \\
& T_{j}^{i}\left(x^{\mu}\right)=\mathcal{P}_{T}\left(x^{\mu}\right)+\Sigma_{j}^{i}\left(x^{\mu}\right)=\sum_{X}\left\{\left[\overline{\mathcal{P}}_{X}+\delta \mathcal{P}_{X}\left(x^{\mu}\right)\right] \delta_{j}^{i}+\Sigma_{j X}^{i}\left(x^{\mu}\right)\right\},
\end{aligned}
$$

onde $\Sigma_{j}^{i}$ é a parte anisotrópica, e com traço nulo $\Sigma_{i}^{i}=0$, da componente espacial do tensor momento energia; e a soma em $X$ é feita sobre os diferentes fluidos presentes no universo.

\subsubsection{Invariança de calibre}

Analogamente ao resultado obtido para as componentes do tensor métrico, a variação das perturbações (sobre a ação do difeomorfismo gerado pela transformação de coordenadas $x \rightarrow \tilde{x}=x+\xi(x)$, com $\xi^{\mu}=\left(\xi^{0}, \xi, i\right)$, onde $\xi^{0}$ e $\xi$ são funções escalares de $\left.x\right)$ do tensor momento energia em dois calibres diferentes está dada pela expressão:

$$
\delta T_{v}^{\mu}=\delta \tilde{T}_{v}^{\mu}+\mathcal{L}_{\xi} \bar{T}_{v}^{\mu}=\delta \tilde{T}_{v}^{\mu}+\xi^{\lambda} \partial_{\lambda} \bar{T}_{v}^{\mu}-\bar{T}_{v}^{\lambda} \partial_{\lambda} \xi^{\mu}+\bar{T}_{\lambda}^{\mu} \partial_{\nu} \xi^{\lambda}
$$


cujas componentes $00,0 i$, ii e $i j$ até primeira ordem são:

$$
\begin{aligned}
\delta \tilde{\mathcal{P}} & =\delta \mathcal{P}-\dot{\mathcal{\mathcal { P }}} \xi^{0}, \\
\delta \tilde{\rho} & =\delta \rho-\dot{\bar{\rho}} \xi^{0}, \\
\tilde{\theta} & =\theta-k^{2} \xi^{0}, \\
\tilde{\Sigma}_{j}^{i} & =\Sigma_{j}^{i} .
\end{aligned}
$$

onde $\theta \equiv \partial_{i} v^{i}$, com o que é possível construir invariantes de calibre que envolvem apenas as perturbações do tensor momento energia:

$$
\begin{aligned}
& \Delta_{1}=\tilde{\Delta}_{1} \equiv \delta \rho-\frac{\dot{\bar{\rho}}}{k^{2}} \theta \\
& \Delta_{2}=\tilde{\Delta}_{2} \equiv \delta \mathcal{P}-c_{s}^{2} \delta \rho \\
& \Delta_{3}=\tilde{\Delta}_{3} \equiv \delta \mathcal{P}-\frac{\dot{\mathcal{P}}}{k^{2}} \theta
\end{aligned}
$$

e invariantes mistos que envolvem perturbações da métrica e do tensor momento energia. Os seguintes são alguns destes invariantes de calibre mistos [Mukhanov et al., 1992]:

$$
\begin{gathered}
\zeta=\tilde{\zeta} \equiv \Phi+\frac{\mathcal{H}}{k^{2}} \Theta=\phi+\frac{\mathcal{H}}{k^{2}} \theta-\frac{k^{2}}{6} \mu, \\
\Theta=\tilde{\Theta} \equiv \theta+k^{2}\left(B-\frac{\dot{\mu}}{2}\right), \\
\Delta \rho=\tilde{\Delta \rho} \equiv \delta \rho+\dot{\bar{\rho}}\left(B-\frac{\dot{\mu}}{2}\right), \\
\Delta \mathcal{P}=\tilde{\Delta \mathcal{P}} \equiv \delta \mathcal{P}+\dot{\mathcal{P}}\left(B-\frac{\dot{\mu}}{2}\right) .
\end{gathered}
$$

Em particular, o invariante de calibre $\zeta$ vai nos ajudar para relacionar a amplitude das perturbações primordiais (após sair do horizonte durante a inflação, no instante em que $k=\mathcal{H}$ ) com a amplitude das perturbações no instante em que entram novamente no horizonte (durante o período de domínio da radiação ou da matéria, no instante em que mais uma vez $k=\mathcal{H}$ ).

Usando estes invariantes, e os invariantes de Bardeen (3.23), é possível reescrever as equações de Einstein (3.27) de uma forma explicitamente invariante de calibre, no espaço de Fourier (onde $\partial_{l} \rightarrow i k_{l}$, e com $k_{l}$ sendo a componente do vetor de onda na direção l) como:

$$
\begin{gathered}
8 \pi G a^{2} \bar{\rho}=3 \mathcal{H}^{2}, \\
8 \pi G a^{2} \overline{\mathcal{P}}=-2 \dot{\mathcal{H}}-\mathcal{H}^{2}, \\
-4 \pi a^{2} G \Delta \rho=k^{2} \Phi+3 \mathcal{H}(\dot{\Phi}+\mathcal{H} \Psi),
\end{gathered}
$$




$$
\begin{gathered}
4 \pi G a^{2}(\bar{\rho}+\overline{\mathcal{P}}) \Theta=k^{2}(\dot{\Phi}+\mathcal{H} \Psi) \\
4 \pi G a^{2} \Delta \mathcal{P}=\ddot{\Phi}+\mathcal{H}(\dot{\Psi}+2 \dot{\Phi})+\left(2 \dot{\mathcal{H}}+\mathcal{H}^{2}\right) \Psi+\frac{k^{2}}{3}(\Phi-\Psi) \\
12 \pi G a^{2}(\bar{\rho}+\overline{\mathcal{P}}) \sigma=k^{2}(\Phi-\Psi) .
\end{gathered}
$$

As duas primeiras expressões são as equações de Einstein não perturbadas, enquanto que as quatro seguintes são respectivamente as componentes tempo-tempo, tempo-espaço, longitudinal espacial, e espacial sem traço das equações de Einstein (2.26) até primeira ordem nas perturbações escalares.

Estas equações são explicitamente invariantes de calibre, e se usarmos as definições dos invariantes (3.23) e (3.33) reobtemos as equações gerais (3.27). Por outro lado, no calibre longitudinal (definido como $B=0$ e $\mu=0$ em (3.7)), estas equações assumem a mesma forma funcional, basta fazer as substituições $\Delta \rho \rightarrow \delta \rho, \Delta \mathcal{P} \rightarrow \delta \mathcal{P}, \Theta \rightarrow \theta, \Psi \rightarrow \psi$ e $\Phi \rightarrow \phi$.

Com frequência é útil expressar as componentes tempo-tempo e tempo-espaço da equação de Einstein como uma equação algébrica para os parâmetros da perturbação da seguinte forma:

$$
4 \pi G a^{2}\left(k^{2} \Delta \rho+3 \mathcal{H}(\bar{\rho}+\overline{\mathcal{P}}) \Theta\right)=-k^{4} \Phi .
$$

Estas equações, junto com as equações de conservação do tensor momento energia (ou as equações de Boltzmann ) constituem um sistema completo de equações diferenciais que determina a evolução temporal das perturbações do tensor métrico e do tensor momento energia.

\section{Perturbação de iso-curvatura}

No caso em que $\sigma=0$ as equações (3.34c) e (3.34d) simplificam para:

$$
\begin{aligned}
-k^{2} \Phi-3 \mathcal{H}(\dot{\Phi}+\mathcal{H} \Phi) & =4 \pi G a^{2} \Delta \rho, \\
\ddot{\Phi}+3 \mathcal{H} \dot{\Phi}+\left(2 \dot{\mathcal{H}}+\mathcal{H}^{2}\right) \Phi & =4 \pi G a^{2} \Delta \mathcal{P},
\end{aligned}
$$

e usando [Mukhanov et al., 1992]:

$$
\Delta \mathcal{P}=\left.\frac{\Delta \mathcal{P}}{\Delta \rho}\right|_{S} \Delta \rho+\left.\frac{\Delta \mathcal{P}}{\Delta S}\right|_{\rho} \Delta S \equiv c_{S}^{2} \Delta \rho+\varsigma \Delta S
$$

as podemos juntar na equação:

$$
\ddot{\Phi}+3 \mathcal{H}\left(1+c_{s}^{2}\right) \dot{\Phi}+c_{s}^{2} k^{2} \Phi+2\left(2 \dot{\mathcal{H}}+\left(1+3 c_{s}^{2}\right) \mathcal{H}^{2}\right) \Phi=4 \pi G a^{2} \tau \delta S
$$

No caso em que $c_{s}^{2} \equiv \Delta \mathcal{P} / \Delta \rho \equiv \delta \mathcal{P} / \delta \rho=\omega \equiv \mathcal{P} / \rho$ o último termo em parêntesis passa a ser identicamente nulo devido às equações de Einstein não perturbadas. Adicionalmente, no caso adiabático 
$(\Delta S=0)$ e para escalas fora do horizonte (onde podemos desconsiderar o termo $\left.c_{s}^{2} k^{2} \Phi\right)$, esta equação pode ser escrita como:

$$
\ddot{\Phi}+3 \mathcal{H}(1+\omega) \dot{\Phi}=0 .
$$

Por outro lado, usando a componente $i 0$ das equações de Einstein (3.34d) podemos reescrever o invariante de calibre $\zeta$ (equação (3.33)) como sendo:

$$
\zeta=\phi+\frac{2}{3} \frac{\phi+a \phi^{\prime}}{1+\omega}
$$

onde a linha denota derivada em relação ao fator de escala. Derivando esta última expressão em relação ao tempo conforme obtemos

$$
3 \mathcal{H}(1+\omega) \dot{\zeta}=2(\ddot{\phi}+3 \mathcal{H}(1+\omega) \dot{\phi})-\left(2 \dot{\mathcal{H}}+(1+3 \omega) \mathcal{H}^{2}\right) \frac{\dot{\phi}}{\mathcal{H}},
$$

onde novamente o último termo em parêntesis é identicamente nulo, enquanto que o primeiro é zero de acordo com a equação (3.39). Com isto

$$
\dot{\zeta}=0
$$

o que significa que: fora do horizonte, em ausência de estresse anisotrópico, e no caso adiabático, o invariante de calibre $\zeta$ (3.33) é conservado. Esta propriedade é comumente usada para relacionar o espectro de potências das perturbações ao sair do horizonte durante a inflação, com o espectro das perturbações quando elas re-entram no horizonte após a inflação.

\subsubsection{Conservação do tensor momento energia}

No caso de um fluido material que interage com o vácuo, as componentes da equação de conservação (2.32) do tensor momento energia perturbado (3.29) podem ser escritas no espaço de Fourier, usando a métrica (3.24) no calibre longitudinal (definido como $B=0$ e $\mu=0$ em (3.7)), da seguinte forma:

$$
\begin{gathered}
\dot{\delta}=-(1+\omega)(\theta-3 \dot{\phi})-3 \mathcal{H}\left(\frac{\delta \mathcal{P}}{\delta \rho}-\omega\right) \delta+\frac{\dot{\bar{\rho}}_{\Lambda}}{\bar{\rho}} \delta, \\
\dot{\theta}=-\mathcal{H}(1-3 \omega) \theta+k^{2} \frac{\delta \mathcal{P} / \delta \rho}{1+\omega} \delta-k^{2} \sigma+k^{2} \psi+\frac{\dot{\bar{\rho}}_{\Lambda}}{\bar{\rho}} \theta,
\end{gathered}
$$

onde usamos $\omega \equiv \mathcal{P} / \rho=(\overline{\mathcal{P}}+\delta \mathcal{P}) /(\bar{\rho}+\delta \rho), \vec{k}$ é o vetor de onda no espaço de Fourier, e definimos as perturbações tempo-tempo, $\delta$, espaço-temporal, $\theta$, e espacial anisotrópica, $\sigma$, para cada fluido como:

$$
\begin{gathered}
\delta \equiv \frac{\delta \rho}{\bar{\rho}}, \quad(\bar{\rho}+\overline{\mathcal{P}}) \theta \equiv i k^{j} \delta T_{j}^{0}, \\
(\bar{\rho}+\overline{\mathcal{P}}) \sigma \equiv-\left(\hat{k}^{i} \hat{k}_{j}-\frac{1}{3} \delta_{j}^{i}\right) \Sigma_{i}^{j}, \quad \Sigma_{i}^{j} \equiv T_{i}^{j}-\frac{\delta_{i}^{j}}{3} T_{k}^{k} .
\end{gathered}
$$

Também usamos, na equação (3.43), $\theta_{\Lambda}=\delta \mathcal{P}_{\Lambda}=\delta_{\Lambda}=\sigma_{\Lambda}=\omega_{\Lambda}+1=0$ no caso das perturbações do vácuo, já que este, a principio, não apresenta o fenômeno de aglomeração, e portanto qualquer pertur- 
bação inicial vai ser rapidamente diluída pela pressão negativa (em geral isto não acontece para escalas fora do horizonte de Hubble, mas a evolução deste tipo de escalas não é invariante de calibre). Para cada um dos fluidos que interagem apenas gravitacionalmente as componentes das equações de conservação do tensor momento energia são dadas pelas expressões (3.43) com $\dot{\bar{\rho}}_{\Lambda}=0$ [Ma and Bertschinger, 1995]:

$$
\begin{gathered}
\dot{\delta}=-(1+\omega)(\theta-3 \dot{\phi})-3 \mathcal{H}\left(\frac{\delta \mathcal{P}}{\delta \rho}-\omega\right) \delta, \\
\dot{\theta}=-\mathcal{H}(1-3 \omega) \theta+k^{2} \frac{\delta \mathcal{P} / \delta \rho}{1+\omega} \delta-k^{2} \sigma+k^{2} \psi .
\end{gathered}
$$

Veremos a seguir as equações de conservação do tensor momento energia no caso específico de um universo com radiação e matéria escura, desconsiderando qualquer interação entre elas.

\section{Radiação}

No caso da radiação (caraterizada pela equação de estado $\overline{\mathcal{P}}_{r} / \bar{\rho}_{r}=1 / 3$ ) obtemos da equação (2.37) que $\bar{\rho}_{r} \propto a^{-4}$, portanto $\dot{\bar{\rho}}_{r}=-4 \bar{\rho}_{r} \mathcal{H}$. Com isto, as equações (3.46a) e (3.46b) tornam-se [Ma and Bertschinger, 1995]:

$$
\dot{\delta}_{r}+\frac{4}{3} \theta_{r}-4 \dot{\phi}=0, \quad \text { e } \quad \dot{\theta}_{r}-k^{2}\left(\frac{\delta_{r}}{4}-\sigma_{r}+\psi\right)=0,
$$

onde usamos $c_{s(r)}^{2} \equiv \delta \mathcal{P}_{r} / \delta \rho_{r} \approx \omega_{r}=1 / 3$. Estas duas equações diferenciais acopladas de primeira ordem podem ser reescritas como equações diferenciais de segunda ordem desacopladas:

$$
\ddot{\delta}_{r}+\frac{k^{2}}{3}\left(\delta_{r}-4 \sigma_{r}+4 \psi\right)-4 \ddot{\phi}=0, \quad \text { e } \quad \ddot{\theta}_{r}+\frac{k^{2}}{3} \theta_{r}-k^{2}\left(\dot{\phi}+\dot{\psi}-\frac{\dot{\sigma}_{r}}{4}\right)=0 .
$$

Por outro lado, durante a o período de domínio da radiação (quando $a \propto \eta$ e portanto $\mathcal{H}=1 / \eta$, ver equação (2.51)) e para escalas fora do horizonte de Hubble $k \ll \mathcal{H}$, por consistência podemos encontrar as condições iniciais para as perturbações usando as mesmas equações de evolução. Sobre tais condições $\dot{\delta}_{r} \sim \delta_{r} / \eta=\delta_{r} \mathcal{H} \gg i k^{i} T_{i}^{0} /\left(\bar{\rho}_{r}+\overline{\mathcal{P}}_{r}\right)=\theta$, a equação (3.47) gera a condição inicial para o contraste de densidade [Ma and Bertschinger, 1995]:

$$
\delta_{r}=4 \phi,
$$

enquanto que a componente $0 i$ da equação de Einstein (3.34d) pode ser aproximada como:

$$
0=4 \pi G a^{2}\left(\bar{\rho}_{r}+\overline{\mathcal{P}}_{r}\right) \theta_{r}-k^{2}(\dot{\phi}+\mathcal{H} \psi) \approx 8 \pi G a^{2} \rho_{r} \frac{2}{3} \theta_{r}-k^{2} \mathcal{H} \psi \approx 2 \mathcal{H}^{2} \theta_{r}-k^{2} \mathcal{H} \psi,
$$

gerando a condição inicial para $\theta_{r}$ [Ma and Bertschinger, 1995]:

$$
\theta_{r}=\frac{k^{2}}{2 \mathcal{H}} \psi \ll \psi
$$




\section{Matéria escura fria}

No caso da matéria, cuja equação de estado é $\overline{\mathcal{P}}_{m} / \bar{\rho}_{m}=0$, a partir da equação (2.40) obtemos que $\bar{\rho}_{m} \propto$ $a^{-3}$, e assim $\dot{\bar{\rho}}_{m}=-3 \bar{\rho}_{m} \mathcal{H}$. Com isto, as equações (3.46a) e (3.46b) tornam-se [Ma and Bertschinger, 1995]:

$$
\dot{\delta}_{m}+\theta_{m}-3 \dot{\phi}=0, \quad \text { e } \quad \dot{\theta}_{m}+\mathcal{H} \theta_{m}-k^{2}\left(\delta_{m} c_{s}^{2}-\sigma_{m}+\psi\right)=0
$$

onde $k^{2} c_{s}^{2}=k^{2} \delta \mathcal{P} / \delta \rho$ não é necessariamente desprezível. Como antes, estas duas equações diferenciais acopladas de primeira ordem na variável temporal podem ser reescritas como uma equação de segunda ordem:

$$
\ddot{\delta}_{m}+\mathcal{H} \dot{\delta}_{m}+k^{2}\left(\delta_{m} c_{s}^{2}-\sigma_{m}+\psi\right)-3 \mathcal{H} \dot{\phi}-3 \ddot{\phi}=0 .
$$

Como no caso das perturbações da radiação, as equações de evolução das perturbações da matéria escura geram de forma auto-consistente as condições iniciais para escalas fora do horizonte $k \ll \mathcal{H}$ durante o período de domínio da radiação. Neste casso, a equação (3.52) gera a seguinte condição inicial para $\delta_{\mathcal{c}}$ [Ma and Bertschinger, 1995]:

$$
\delta_{c}=3 \phi
$$

\subsubsection{Função de correlação e espectro de potências}

Na modelagem apresentada anteriormente, a sobredensidade de matéria é descrita pelas perturbações na densidade de energia da matéria parametrizada no contraste de densidade:

$$
\delta(\vec{x}, t)=\frac{\rho-\bar{\rho}}{\bar{\rho}}=\frac{\delta \rho}{\bar{\rho}}
$$

é definido para ser uma função suave, onde $\bar{\rho}=\bar{\rho}(t)$ é a média espacial da densidade de energia para um dado valor do tempo. No mundo real, o único que podemos fazer é contar o número de galáxias no céu, e no máximo levar em conta a massa de cada uma delas. Para descrever a distribuição de matéria que podemos quantificar como um campo discreto da seguinte forma:

$$
\delta_{g}(\vec{x}, t)=\frac{n_{g}-\bar{n}_{g}}{\bar{n}_{g}}=\frac{\delta n_{g}}{\bar{n}_{g}},
$$

onde $n_{g}(\vec{x}, t) \equiv d N_{g} / d V$ é o número de galáxias em um volume $V$ em torno do ponto $\vec{x}$ e no instante de tempo $t$. Estas duas quantidades (o contraste de densidade de energia da matéria $\delta$, e o contraste da densidade numero de galáxias $\delta_{g}$ ) estão relacionadas fenomenologicamente por

$$
\delta_{g}=b \delta
$$

onde o parâmetro $b$ é conhecido como bias.

Dado que não podemos predizer qual vai ser o contraste de densidade em um ponto dado do céu $x^{\mu}$, devemos recorrer à análise das suas propriedades estatísticas, que por outro lado também podem ser 
quantificadas na sua contraparte observacional da seguinte forma: podemos contar o numero de pares de galaxias no céu separadas por uma distância $r$, para um valor fixo do tempo, como:

$$
\begin{aligned}
\left\langle d N_{1}(\vec{x}) d N_{2}(\vec{x}+\vec{r})\right\rangle_{\vec{x}(r)} & =\left\langle n_{g}(\vec{x}) d V_{1} n_{g}(\vec{x}+\vec{r}) d V_{2}\right\rangle_{\vec{x}}= \\
& =\left\langle\bar{n}_{g}(\vec{x})\left(1+\delta_{g}(\vec{x})\right) \bar{n}_{g}(\vec{x}+\vec{r})\left(1+\delta_{g}(\vec{x})\right)\right\rangle_{\vec{x}} d V_{1} d V_{2}= \\
& =\left\langle 1+\delta_{g}(\vec{x})+\delta_{g}(\vec{x})+\delta_{g}(\vec{x}) \delta_{g}(\vec{x}+\vec{r})\right\rangle_{\vec{x}} \bar{n}_{g}^{2} d V_{1} d V_{2}= \\
& =\left\langle\delta_{g}(\vec{x}) \delta_{g}(\vec{x}+\vec{r})\right\rangle_{\vec{x}} \bar{n}_{g}^{2} d V_{1} d V_{2}= \\
& =\langle\delta(\vec{x}) \delta(\vec{x}+\vec{r})\rangle_{\vec{x}} b^{2} \bar{n}_{g}^{2} d V_{1} d V_{2},
\end{aligned}
$$

onde a média é feita sobre o espaço real para um $t$ fixo. Com isto, a função de correlação de pares de galáxias (lado esquerdo da equação (3.58)) fica relacionada (por intermédio da equação anterior) com a função de correlação $\xi(r)$ para a sobredensidade de matéria em dois pontos separados por uma distância $r$, definida como:

$$
\xi(r) \equiv\langle\delta(\vec{x}) \delta(\vec{x}+\vec{r})\rangle_{\vec{x}}
$$

Assim, podemos comparar o estimativo estatístico para a contagem de galáxias no ceu com as predições da teoria de perturbações linear para a sobredensidade de matéria. Isso é feito usualmente no espaço de Fourier, definindo o espectro de potência $P(\vec{k}, \eta)$ da matéria como a transformada de Fourier da função de correlação do contraste de densidade de dois pontos $\xi(r)$ da seguinte forma:

$$
\left\langle\delta_{m}^{*}(\vec{k}, \eta) \delta_{m}\left(\vec{k}^{\prime}, \eta\right)\right\rangle \equiv(2 \pi)^{3} \mathrm{P}(k, \eta) \delta^{3}\left(\vec{k}-\vec{k}^{\prime}\right)
$$

com $\delta_{m}$ é o contraste de densidade de energia total da matéria:

$$
\delta_{m} \equiv \frac{\sum_{X} \bar{\rho}_{X} \delta_{X}}{\sum_{X} \bar{\rho}_{X}}
$$

onde o sub-índice $X$ é somado sobre todos os fluidos massivos, que no modelo padrão são a matéria escura fria, os bárions e os neutrinos massivos. O fator $\delta^{3}\left(\vec{k}-\vec{k}^{\prime}\right)$ surge devido à independência na evolução dos modos $\left\{\vec{k}, \vec{k}^{\prime}\right\}$ na teoria de perturbações lineares.

Mais para frente veremos que o espectro de potências primordial (após o período inflacionário) pode se parametrizado como uma potência de ordem $n_{s}-1$ no numero de onda $k$ :

$$
\Delta_{0}(k) \equiv \frac{k^{3}}{2 \pi^{2}} \mathrm{P}_{0}(k) \propto\left(\frac{k}{\mathcal{H}_{0}}\right)^{n_{s}-1},
$$

onde $n_{s} \approx 0.96$ de acordo com as observações [Spergel et al., 2007; Percival, 2007; Komatsu et al., 2009, 2011; Planck Collaboration, 2014]. $n_{s}=1$ corresponderia a um espectro de potência primordial invariante de escala, predito pelos modelos com uma inflação exponencial (inflação de Sitter), enquanto os modelos de inflação usando campos escalares predizem $n_{s}=1-3 \epsilon-\lambda$. $\epsilon \ll 1$ e $\lambda \ll 1$ são os parâmetros que definem a condição conhecida como rolamento lento (ver seção 4.1, e equações (4.13a) e $(4.13 b))$, que por sua vez garante as condições que devem ser satisfeitas para produzir um período inflacionário que resolva o problema do horizonte. 
É de destacar que as quantidades perturbadas (tais como $\delta \rho, \delta \mathcal{P}$ e $\theta$ ) não são invariantes de calibre para escalas de super-horizonte $(k<\mathcal{H}$, ver equação (3.32)) [Ma and Bertschinger, 1995].

\subsection{PERTURBAÇÕES TENSORIAIS}

As perturbações tensoriais lineares com $\delta T^{\mu v}=0$ comportam-se como ondas que viajam com a velocidade da luz, e são conhecidas como ondas gravitacionais.

$$
g_{\mu v}=a^{2}\left(\begin{array}{cccc}
-1 & 0 & 0 & 0 \\
0 & & & \\
0 & \delta_{i j}+f^{T} \\
0 & & &
\end{array}\right)
$$

onde $\hbar_{i j}^{T}$ é um tensor $3 \times 3$ simétrico, sem traço e com $\partial^{i} \hbar_{i j}^{T}=0$, o que deixa ele com dois graus de liberdade. Para este tensor métrico as componentes da conexão diferentes de zero são:

$$
\begin{aligned}
\Gamma_{00}^{0}=\mathcal{H}, & \Gamma_{j 0}^{i}=\mathcal{H} \delta_{i j}+\frac{\dot{h}_{i j}^{T}}{2}, \\
\Gamma_{i j}^{0}=\mathcal{H}\left(\delta_{i j}+\hbar_{i j}^{T}\right)+\frac{\dot{h}_{i j}^{T}}{2}, & \Gamma_{j k}^{i}=\frac{1}{2}\left(\epsilon_{k i, j}^{T}+\kappa_{i j, k}^{T}-\kappa_{j k, i}^{T}\right),
\end{aligned}
$$

e as componentes de ordem zero (nas perturbações) do tensor de Ricci ficam escritas como

$$
\begin{gathered}
R_{00}=-3 \dot{\mathcal{H}}, \quad R_{i 0}=\frac{\dot{h}_{i j, j}^{T}}{2}=\frac{\dot{h}_{i j, j}^{T}}{2} \equiv 0, \\
R_{i j}=\left(\dot{\mathcal{H}}+2 \mathcal{H}^{2}\right)\left(\delta_{i j}+h_{i j}^{T}\right)+\mathcal{H} \dot{h}_{i j}^{T}+\frac{\ddot{h}_{i j}^{T}}{2}-\frac{\hat{h}_{i j, k k}^{T}}{2},
\end{gathered}
$$

de onde encontramos que $R \equiv g^{\mu v} R_{\mu v}=0$. Com isto, a componente $i j$ das equações de Einstein é:

$$
a^{2} G_{j}^{i}=-\delta_{i j}\left(2 \dot{\mathcal{H}}+\mathcal{H}^{2}\right)+\mathcal{H} \dot{h}_{i j}^{T}+\frac{\ddot{h}_{i j}^{T}}{2}-\frac{\kappa_{i j, k k}^{T}}{2} .
$$

\subsubsection{Ondas gravitacionais}

No espaço de fase o número de partículas de uma determinada espécie de fluido em um diferencial de volume $d x^{1} d x^{2} d x^{3} d P_{1} d P_{2} d P_{3}$ esta dado pela sua função distribuição da seguinte forma:

$$
f\left(x^{i}, p_{j}, \eta\right) d x^{1} d x^{2} d x^{3} d P_{1} d P_{2} d P_{3}=d N
$$

igualmente, no espaço de fase podemos calcular o tensor momento energia como sendo:

$$
T_{v}^{\mu}=\int d P_{1} d P_{2} d P_{3}(-g)^{-1 / 2} \frac{P^{\mu} P_{v}}{P^{0}} f\left(x^{i}, p_{j}, \tau\right),
$$

onde $f\left(x^{i}, p_{j}, \tau\right)$ é a função distribuição estatística do fluido em consideração, $P_{i}$ é o momento conjugado da coordenada comóvel $x^{i}$. Este momento conjugado $P_{i}$ é simplesmente a componente espacial do 
quadrivetor momento $P^{\mu}=m U^{\mu}$. Agora, se reescrevermos $f\left(x^{i}, p_{j}, \tau\right)$ como um termo não perturbado mais uma pequena perturbação:

$$
f\left(x^{i}, p_{j}, \tau\right) \equiv \bar{f}(p, \tau)\left[1+\Psi\left(x^{i}, \hat{p}, \tau\right)\right]
$$

$\operatorname{com} \bar{f}(q, \tau)$ sendo a função distribuição não perturbada independente do ponto $x^{i}$, onde é calculada, e da direção do momento $\hat{p}_{i}$ do fluido nesse ponto:

$$
\bar{f}(p, \tau)=\frac{g_{s}}{(2 \pi)^{3}} \frac{1}{e^{(E-\mu) / k_{B} T} \pm 1}
$$

onde $g_{s}$ é o nível de degenerescência quântico da espécie em consideração, $\mathrm{T}$ é a temperatura, $E=$ $\sqrt{p^{2}+m_{0}^{2}}$ e $\mu$ são a energia e o potencial químico, $m_{0}$ é a massa, e $p$ é a magnitude da componente espacial $P^{i}$ do quadrimomento $P$ definido como:

$$
P^{2} \equiv g_{\mu \nu} P^{\mu} P^{v}=g_{00} P^{0} P^{0}+p^{2}=m_{0}^{2}
$$

Em um sistema de coordenadas localmente inercial, e para um fluido ultra-relativístico, as componentes espaciais do tensor momento energia são:

$$
T_{j}^{i}=\int d^{3} p \frac{p^{i} p_{j}}{p}\left[1-\frac{\delta \mathrm{T}\left(x^{i}, \hat{p}, \tau\right)}{\overline{\mathrm{T}}} \frac{\partial}{\partial \ln p}\right] \bar{f}(p, \tau),
$$

assim, em coordenadas esféricas, para uma temperatura do fluido independente de $\varphi$, obtemos:

$$
T_{r}^{r}-T_{\theta}^{\theta}=-\int d p p^{3} \frac{\partial}{\partial p} \bar{f}(p, \tau) \times \int d \cos (\theta) \sin ^{2}(\theta) \int d \varphi\left(\cos ^{2}(\varphi)-\sin ^{2}(\varphi)\right) \frac{\delta \mathrm{T}\left(x^{i}, \hat{p}, \tau\right)}{\overline{\mathrm{T}}}
$$

No caso em que $\delta$ T independe de $\varphi$ a última integral é nula, produzindo:

$$
T_{r}^{r}=T_{\theta}^{\theta}=-\frac{3 \pi}{2} \bar{T}^{2} g_{s} \int_{-1}^{1} d \mu\left(1-\mu^{2}\right) \delta \mathrm{T}\left(x^{i}, \mu, \tau\right),
$$

onde $g_{s}$ é o grau de degenerescência da espécie em consideração multiplicado por $(3,4)$ para (bósons,férmions). Como também obtemos:

$$
T_{\theta}^{r}=-\frac{3}{2} \bar{T}^{2} g_{s} \int_{-1}^{1} d \mu\left(1-\mu^{2}\right) \delta \mathrm{T}\left(x^{i}, \mu, \tau\right) \int d \theta \sin ^{2}(\theta) \cos (\theta)=0
$$

Uma forma simples de escrever os 2 graus de liberdade das perturbações tensoriais vem dada pela seguinte parametrização:

$$
\boldsymbol{h}_{i j}^{T}=\left(\begin{array}{ccc}
\hbar_{+} & h_{\times} & 0 \\
\hbar_{\times} & -\hbar_{+} & 0 \\
0 & 0 & 0
\end{array}\right)
$$

devido a que a última integral é identicamente nula.

Assim, durante os estágios primordiais do universo, no caso em que a temperatura dos fluidos não depende de $\varphi$ (ângulo azimutal em coordenadas esféricas) obtemos que $T_{r}^{r}-T_{\theta}^{\theta}=T_{\theta}^{r}=0$. Usando a 
equação (3.64) podemos obter a equação de evolução para as perturbações tensoriais na aproximação linear e no espaço de Fourier como sendo:

$$
\begin{aligned}
a^{2}\left(G_{r}^{r}-G_{\theta}^{\theta}\right) & =0=\ddot{h}_{+}+2 \mathcal{H} \dot{h}_{+}+k^{2} h_{+}, \\
2 a^{2} G_{\theta}^{r}=0 & =\ddot{h}_{\times}+2 \mathcal{H} \dot{h}_{\times}+k^{2} h_{\times},
\end{aligned}
$$

onde $k$ é o número de onda no espaço de Fourier. Estas equações tem a forma de uma equação de onda sem fontes, e sua solução (conhecida como ondas gravitacionais) se propaga na direção de $\theta=0$, com a velocidade da luz.

No caso geral em que $8 \pi G a^{2}\left(T_{r}^{r}-T_{\theta}^{\theta}\right) \neq 0$ ou $16 \pi G a^{2} T_{\theta}^{r} \neq 0$, estas duas quantidades representam as fontes para as ondas gravitacionais $h_{r r} \equiv h_{+}$e $h_{r \theta} \equiv h_{\times}$, respectivamente. 



\section{IN F L A Ç Ã O}

As observações da radiação cósmica de fundo [Spergel et al., 2003, 2007; Komatsu et al., 2009, 2011] mostram que a temperatura dos fótons que chegam de todas as direções do céu é muito uniforme. Este resultado não era esperado pelos modelos com um Big Bang quente tipo $\Lambda$ CDM, já que durante a última superfície de espalhamento (que é a época da que recebemos hoje a radiação cósmica de fundo, e está localizada dentro do período de domínio da matéria) qualquer duas regiões separadas por uma distância maior que o raio comóvel de Hubble naquela época $\left(\mathcal{H}_{l s}^{-1}\right)$ nunca estiveram em contato térmico, já que o raio comóvel de Hubble decresce a medida que o universo se expande durante os períodos de domínio da radiação e da matéria escura. Este é o chamado problema do horizonte do modelo $\Lambda$ CDM, que pode ser resolvido introduzindo um período de expansão acelerado anterior ao período de domínio da radiação, como veremos aseguir.

Um universo em expansão, homogêneo, isotrópico e espacialmente plano em grandes escalas pode ser descrito em coordenadas comoveis, e usando o tempo conforme, pela métrica (2.16). Neste contexto, desde o começo do universo um fóton pode viajar, em coordenadas comoveis, uma distância igual ao tempo conforme

$$
\eta=\int_{0}^{t} \frac{d \breve{t}}{a(\breve{t})}=\int_{0}^{a} \frac{d t(\breve{a})}{d \breve{a}} \frac{d \breve{a}}{\breve{a}}=\int_{0}^{a} \frac{d \breve{a}}{\breve{a}^{2} H} .
$$

Na fase em que o universo é dominado por um fluido com pressão nula as equações de Einstein (2.48) fornecem uma evolução do fator de escala da forma (2.53):

$$
a(\eta)=a_{0}\left(\frac{\eta}{\eta_{0}}\right)^{2}, \quad \eta=\eta_{0} \sqrt{\frac{a}{a_{0}}}, \quad \mathcal{H}=\frac{2}{\eta},
$$

obtendo que o raio de Hubble aumenta com o decorrer do tempo. Com isto, a nossa distância até a última superfície de espalhamento dos fótons (superfície esférica ao redor nosso em $z \approx 1100$ ou $\eta \equiv \eta_{l s}$ ) é

$$
r_{l s} \equiv \eta_{0}-\eta_{l s}=\eta_{0}\left(1-\sqrt{\frac{a_{l s}}{a_{0}}}\right)
$$

enquanto o raio comóvel de Hubble naquele momento é

$$
\mathcal{H}_{l s}^{-1}=\frac{\eta_{0}}{2} \sqrt{\frac{a_{l s}}{a_{0}}}, \quad \mathcal{H} \equiv a H,
$$

usando $a_{l s} \approx 1 / 1100$ obtemos que

$$
\frac{r_{l s}}{\mathcal{H}_{l s}^{-1}} \approx 66.3 \approx 10^{2} .
$$


Isso que dizer que em $z \approx 1100$ (durante a última superfície de espalhamento dos fótons, a partir da que eles viajam livres até nos) somente regiões com comprimento característico $\sim 100$ vezes menor que o céu de fótons que hoje observamos (fótons emitidos naquela época) conseguiam trocar informação -por causa da expansão do universo- para se termalizar. E o que é pior ainda: para $z>1100$ as regiões em contato térmico eram menores, já que para um universo espacialmente plano e dominado por um fluido com equação de estado $\mathcal{P}=\omega \rho$, o raio comóvel de Hubble (2.50) cresce durante a expansão se o fluido dominante é descrito por $\omega>-1 / 3$ :

$$
\mathcal{H}^{-1}=\frac{1+3 \omega}{2} \eta+c_{1}, \quad \rightarrow \quad \partial_{\eta} \mathcal{H}^{-1}=\frac{1+3 \omega}{2}
$$

Em resumo, se voltarmos no tempo as regiões em contato térmico (limitadas pelo comprimento do raio comóvel de Hubble) são cada vez menores para um universo dominado por fluidos com pressão nula ou positiva (é o caso do modelo $\Lambda \mathrm{CDM}$ para períodos anteriores à última superfície de espalhamento). Sendo assim, se o universo foi dominado por fluidos relativísticos $(\omega \approx 1 / 3)$ a partir do big bang, nós conseguiríamos ver no céu (ver equação $(4.5)) \sim 10^{4}$ regiões angulares diferentes com temperaturas independentes, mas o que as observações apresentam para nós [Spergel et al., 2003, 2007; Komatsu et al., 2009, 2011] é um céu, com uma temperatura uniforme com uma precisão de 5 casas decimais.

Isto sugere que o universo teve uma era (anterior ao domínio da matéria e da radiação) onde o raio comóvel de Hubble diminuiu enquanto o universo expandia. Assim, antes daquele período, que chamaremos de inflação por razões que ficarão claras mais para frente (ver equações (4.8-4.9)), o raio comóvel de Hubble possuía um comprimento maior ou igual ao raio da última superfície de espalhamento dos fótons $r_{l s}$, de tal forma que regiões com dito tamanho conseguiram estabelecer um equilíbrio térmico. Eventualmente estas regiões ficaram fora do horizonte, mas as perturbações na temperatura dos fótons permaneceram congeladas durante a maior parte de dito estágio, permitindo que na sua recente reentrada no horizonte na época da última superfície de espalhamento, ainda apresentem a uniformidade que térmica observamos.

Vamos então procurar as condições que permitam ao universo passar por um período de expansão onde o raio comóvel de Hubble decresça com a evolução temporal. Das equações de Einstein (2.44) para um universo plano descrito pela métrica (2.16) e contendo $M$ fluidos podemos obter

$$
-6 \dot{\mathcal{H}}=8 \pi G\left(3 \overline{\mathcal{P}}_{T}+\bar{\rho}_{T}\right)=8 \pi G \sum_{X=1}^{M}\left(3 \overline{\mathcal{P}}_{X}+\bar{\rho}_{X}\right)
$$

com a equação acima podemos ver que o raio comóvel de Hubble decresce com a expansão do universo $\partial_{t} \mathcal{H}^{-1}=-\dot{\mathcal{H}} /\left(a \mathcal{H}^{2}\right)<0$ sempre que

$$
\sum_{X=1}^{M}\left(3 \overline{\mathcal{P}}_{X}+\bar{\rho}_{X}\right)<0 .
$$

Isto é possível somente se o universo for dominado por um fluido com a propriedade $3 \overline{\mathcal{P}}<-\bar{\rho}$ durante um estágio anterior à era de domínio da radiação. A condição (4.8) também implica que durante o 
período de inflação a expansão do universo acontece de forma acelerada, já que a equação (4.7) reescrita em função do tempo cósmico é:

$$
\frac{\partial^{2} a}{\partial t^{2}}=-\frac{4 \pi G a}{3} \sum_{X=1}^{M}\left(3 \overline{\mathcal{P}}_{X}+\bar{\rho}_{X}\right)
$$

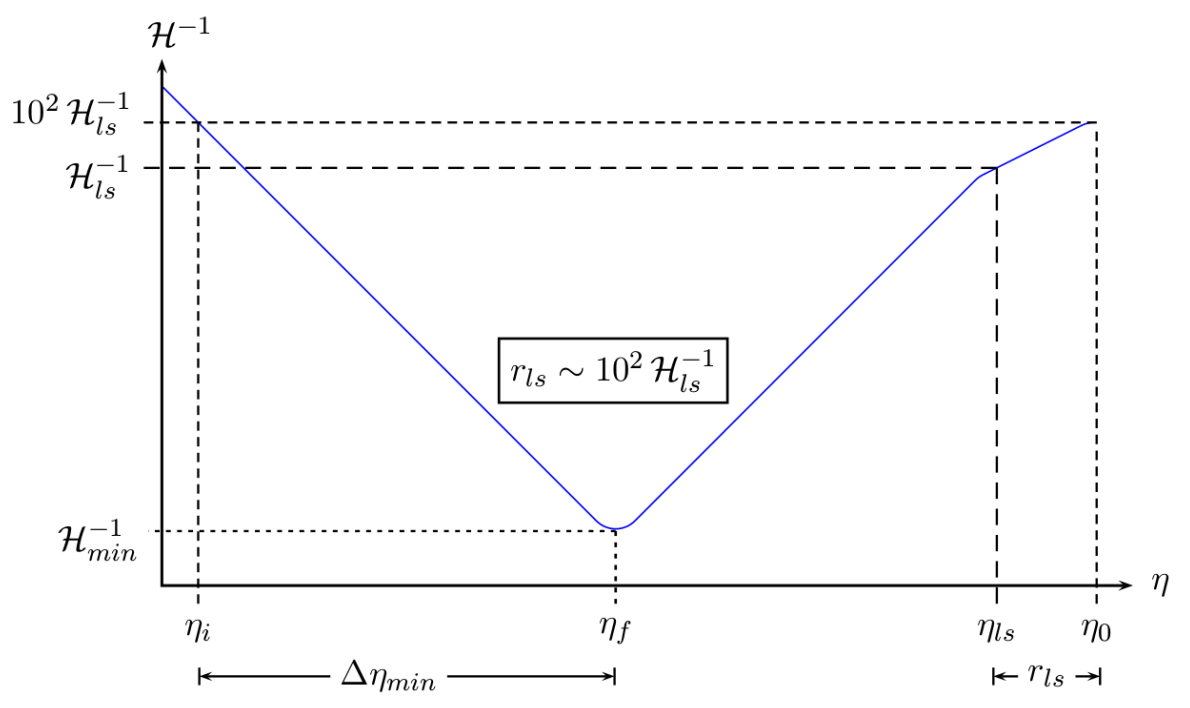

Figura 4.1: Solução da inflação ao problema de horizonte.

Esquema $\log \times \log$ da forma como um período inflacionário resolve o problema de horizonte. $r_{l s}$ é o raio comóvel da última superfície de espalhamento, e $\mathcal{H}_{l s}$ é o raio comóvel de Hubble durante a última superfície de espalhamento [Dodelson, 2003].
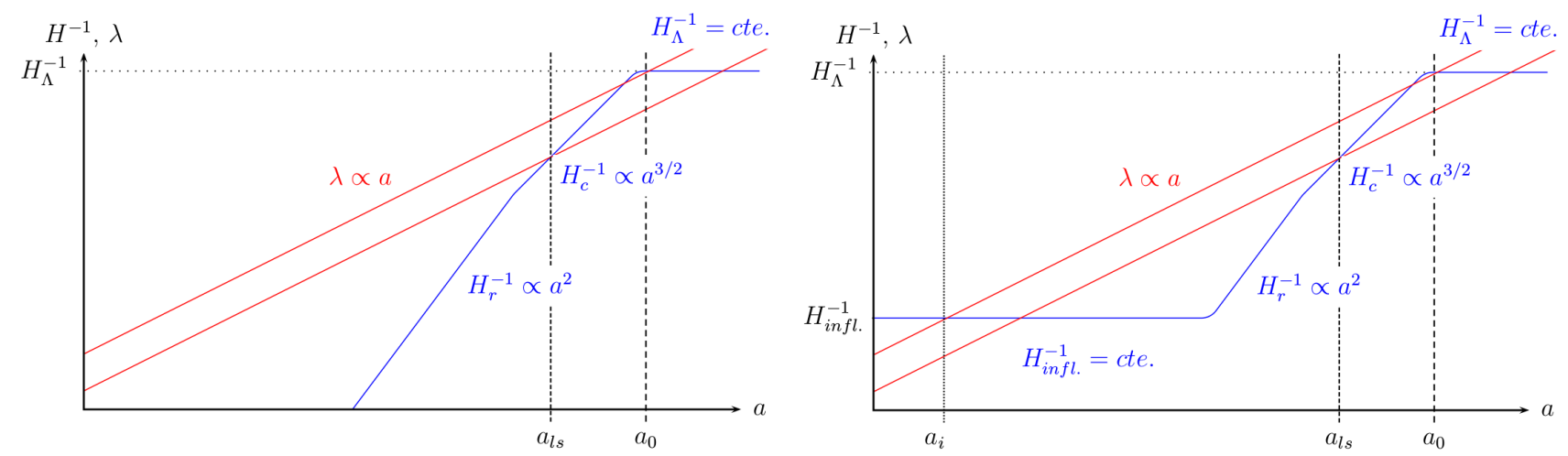

Figura 4.2: Solução da inflação ao problema de horizonte.

O painel esquerdo mostra como as escalas físicas que estavam fora do horizonte na última superfície de espalhamento (em vermelho) tinham permanecido sempre fora horizonte (curva azul) para um universo inicialmente dominado por radiação. O painel direito mostra como um estágio inicial acelerado (anterior ao período de domínio da radiação, e que deve começar para $a \leqslant a_{i}$ ) resolve o problema do horizonte, já que as escalas que observamos hoje e que estavam fora do horizonte na última superfície de espalhamento, estiveram todas dentro do horizonte para $a<a_{i}$.

A condição (4.8) é necessária para que aconteça a inflação, mas não é suficiente para resolver o problema de horizonte. Para resolvê-lo é preciso que o período de inflação seja longo o suficiente, de tal forma que $\mathcal{H}_{i}^{-1} / \mathcal{H}_{f}^{-1}$ seja maior ou pelo menos igual a $10^{2} \mathcal{H}_{0}^{-1} / \mathcal{H}_{f}^{-1}$ (ver a equação (4.5) e a figura 4.1 ), onde 
$\mathcal{H}_{i}^{-1}, \mathcal{H}_{f}^{-1}$ e $\mathcal{H}_{0}^{-1}$ são respectivamente os raios comoveis de Hubble no começo da inflação, no fim da inflação e hoje.

Vamos estimar a duração mínima da inflação para que esta resolva o problema do horizonte no caso de um universo dominado pelo vácuo $\mathcal{P}_{\Lambda}=-\rho_{\Lambda}$ (o que gera um crescimento exponencial do fator de escala em função do tempo cósmico, ver equação (2.56)) durante o período inflacionário até a escala de energia da grande unificação $\left(10^{16} \mathrm{GeV}\right)$, e dominado pela radiação até a última superfície de espalhamento dos fótons (já que a última superfície de espalhamento $z \approx 1100$ acontece pouco depois da transição radiação-matéria em $z \approx 5263$ [Beringer et al., 2012] no modelo padrão).

Para um universo dominado por radiação, matéria e constante cosmológica o parâmetro de Hubble evolui na forma (2.52):

$$
H(a)=H_{0} \sqrt{\Omega_{\Lambda}+\Omega_{m}\left(a / a_{0}\right)^{-3}+\Omega_{\gamma}\left(a / a_{0}\right)^{-4}},
$$

e dado o problema da constante cosmológica $H_{g u t} \approx 10^{53} H_{0}$ podemos aproximar $a_{g u t}=2.6 \times 10^{-28} a_{0}$. O sub-índice gut avalia a quantidade de interesse no instante em que o universo atinge a escala de energia da grande unificação; enquanto que o sub-índice 0 denota o instante atual da história cósmica. Assim, o quociente entre o comprimento comóvel característico do céu observado hoje $10^{2} \mathcal{H}_{l s}^{-1}$ (todo com uma mesma temperatura) e o raio comóvel de Hubble logo após a inflação $\mathcal{H}_{g u t}^{-1}$ é:

$$
\frac{10^{2} \mathcal{H}_{l s}^{-1}}{\mathcal{H}_{\text {gut }}^{-1}}=\frac{10^{2} H_{\text {gut }} a_{g u t}}{H_{l s} a_{l s}} \approx 5 \frac{H_{g u t} a_{g u t}}{H_{0} a_{0}} \approx 10^{26},
$$

onde usamos $H_{l s} \approx 2 \times 10^{4} H_{0}$ e $a_{l s} \approx 1 / 1101$. Durante uma inflação gerada pelo domínio do vácuo $a=a_{i} e^{H_{I} t}$ (ver (2.56), onde $H_{I}$ é o parâmetro de Hubble, que permanece constante durante uma inflação dominada pelo vácuo), com isto podemos escrever o quociente entre o raio comóvel de Hubble no começo e no fim da inflação como

$$
\frac{\mathcal{H}_{i}^{-1}}{\mathcal{H}_{f}^{-1}}=\frac{a_{f}}{a_{i}}=e^{H_{I}\left(t_{f}-t_{i}\right)} \equiv e^{e_{0}}=10^{26}, \quad e_{0} \approx 59.9,
$$

onde o quociente dos raios foi igualado a (4.11) para resolver o problema de horizonte, e $e_{0}$ é o chamado número mínimo de e-folds que deve durar o período inflacionário. Se consideramos a escala de Planck como sendo a escala da inflação obtemos $e_{0} \approx 66.8$ e $a_{p l}=2.6 \times 10^{-31} a_{0}$.

\subsection{PARÂMETROS DE ROLAMENTO LENTO}

Vamos escrever parâmetros gerais que demarcados adequadamente caracterizam as condições adequadas para que a inflação resolva o problema do horizonte. A primeira condição para que a inflação aconteça é a negatividade da derivada do raio comóvel de Hubble:

$$
\frac{d}{d \eta}\left(\mathcal{H}^{-1}\right)=a \frac{d}{d t}\left(\frac{1}{a H}\right)=\frac{d H^{-1}}{d t}-1 \equiv \epsilon-1<0, \quad \epsilon \equiv 1-\frac{\dot{\mathcal{H}}}{\mathcal{H}^{2}}
$$

assim, para que o período inflacionário aconteça devemos ter $\epsilon<1$. Mas, para que o período inflacionário seja longo o suficiente (para resolver o problema do horizonte) podemos exigir que $\epsilon \ll 1$ 
inicialmente (no começo da inflação), e ainda que demore muito para chegar em $\epsilon=1$ (fim da inflação), o que podemos medir pelo parâmetro

$$
\left(\frac{1}{a} \frac{d a}{d \eta}\right)^{-1}\left(\frac{1 d \epsilon}{\epsilon} \frac{d}{d \eta}\right)=\mathcal{H}^{-1} \frac{d \ln \epsilon}{d \eta}=\frac{d \ln \epsilon}{d \ln a} \equiv \lambda \ll 1 .
$$

Assim, a inflação ocorre se $\epsilon<1$, e consegue se manter por suficiente tempo se $\epsilon \ll 1$ e $\lambda \equiv$ $d \ln \epsilon / d \ln a \ll 1$. Estes são os chamados parâmetros slow roll.

\subsection{IMPLEMENTAÇÃO DA INFLAÇÃO USANDO UM CAMPO ESCALAR}

Uma maneira simples de gerar um período de expansão acelerada (inflação) é modelar o universo primordial como sendo dominado por um campo escalar $\varphi$ com energia potencial muito maior que sua energia cinética. Esta energia potencial pode ser gerada por auto interação, não sendo necessária a inclusão de campos externos no modelo. Começamos por escrever a lagrangiana para um campo escalar com potencial de interação $V$ :

$$
\mathcal{L}=-\frac{1}{2} g^{\mu v} \varphi, \mu \varphi, v-V(\varphi)
$$

Agora, usando a expressão que descreve o tensor momento energia para a lagrangiana acima

$$
T_{\mu \nu}=\varphi_{, \mu} \varphi_{, v}-g_{\mu \nu}\left(\frac{g^{\alpha \beta}}{2} \varphi_{, \alpha} \varphi_{, \beta}+V(\varphi)\right)
$$

podemos calcular a pressão e a densidade de energia associadas ao campo escalar dentro de um universo plano descrito pela métrica FRW (2.16):

$$
\rho=\frac{1}{2 a^{2}}\left(\dot{\varphi}^{2}+2 a^{2} V\right), \quad \mathcal{P}=\frac{1}{2 a^{2}}\left(\dot{\varphi}^{2}-2 a^{2} V\right) .
$$

Lembrando que a condição para que aconteça o período inflacionário é que exista no universo um fluido dominante com a propriedade $\mathcal{P}<-\rho / 3$, podemos concluir que o nosso campo escalar é capaz de gerar tal período de expansão acelerada se apresentar um estado tal que, durante este, sua energia cinética seja bem menor que a sua energia de interação. No casso particular em que $\left(\partial_{t} \varphi\right)^{2} \ll V$ conseguimos o limite da inflação exponencial $\mathcal{P} \approx-\rho$, que equivale a termos a condição $\epsilon \ll 1$ satisfeita. Isto pode ser visto através das equações de Einstein

$$
8 \pi G\left(\frac{\dot{\varphi}^{2}}{2}+a^{2} V(\varphi)\right)=3 \mathcal{H}^{2}, \quad 8 \pi G\left(\frac{\dot{\varphi}^{2}}{2}+a^{2} V(\varphi)\right)=-2 \dot{\mathcal{H}}-\mathcal{H}^{2},
$$

de onde podemos obter:

$$
\epsilon=\frac{3(\rho+\mathcal{P})}{2 \rho}=\frac{3 \dot{\varphi}^{2}}{\dot{\varphi}^{2}+2 a^{2} V}
$$

assim, $\epsilon \ll 1$ sempre que $\dot{\varphi}^{2} \ll 2 a^{2} V$, o que implica em $P \approx-\rho \approx-V$, gerando uma inflação exponencial. 
Antes de preocuparmos com o segundo parâmetro de rolamento lento, $\lambda$ (4.13b), podemos usar a componente temporal da equação de conservação do tensor momento energia do campo escalar, $T_{; \mu}^{\mu \nu}=0$ (com a métrica (2.16)), para obter a equação de evolução do campo escalar:

$$
\ddot{\varphi}+2 \mathcal{H} \dot{\varphi}+a^{2} V_{, \varphi}=0,
$$

onde $V_{, \varphi} \equiv \partial V / \partial \varphi$. Já que a condição para que a inflação ocorra é dada pela restrição $\dot{\varphi}^{2} \ll 2 a^{2} V$, e estamos querendo que a inflação seja longa o suficiente, é preciso exigir que $\ddot{\varphi} \approx 0$. Usando esta condição na equação anterior e substituindo-lha na expressão (4.18) obtemos

$$
\epsilon=\frac{9}{64 \pi G}\left(\frac{V_{, \varphi}}{V}\right)^{2} \ll 1,
$$

onde podemos ver que a condição para que exista o período inflacionário durante o domínio do campo escalar é que o potencial $V$ varie lentamente com a evolução do campo escalar $\varphi$. Derivando mais uma vez a expressão anterior podemos encontrar a seguinte forma para o parâmetro $\lambda$ :

$$
\lambda \equiv \frac{\dot{\epsilon}}{\mathcal{H} \epsilon}=\frac{3}{8 \pi G}\left(\left(\frac{V, \varphi}{V}\right)^{2}-\frac{V_{, \varphi \varphi}}{V}\right)=-\frac{3}{8 \pi G} \partial_{\varphi}^{2} \ln V \ll 1 .
$$

Podemos então concluir que para obter uma inflação duradoura e gerada por um campo escalar com potencial $V$ devemos exigir que

$$
M_{p l} V_{, \varphi} \ll V, \quad \text { como também } \quad M_{p l}^{2} V_{, \varphi \varphi} \ll V,
$$

onde $M_{p l}$ é a massa de Planck.

\subsubsection{Perturbações do campo escalar inflacionário}
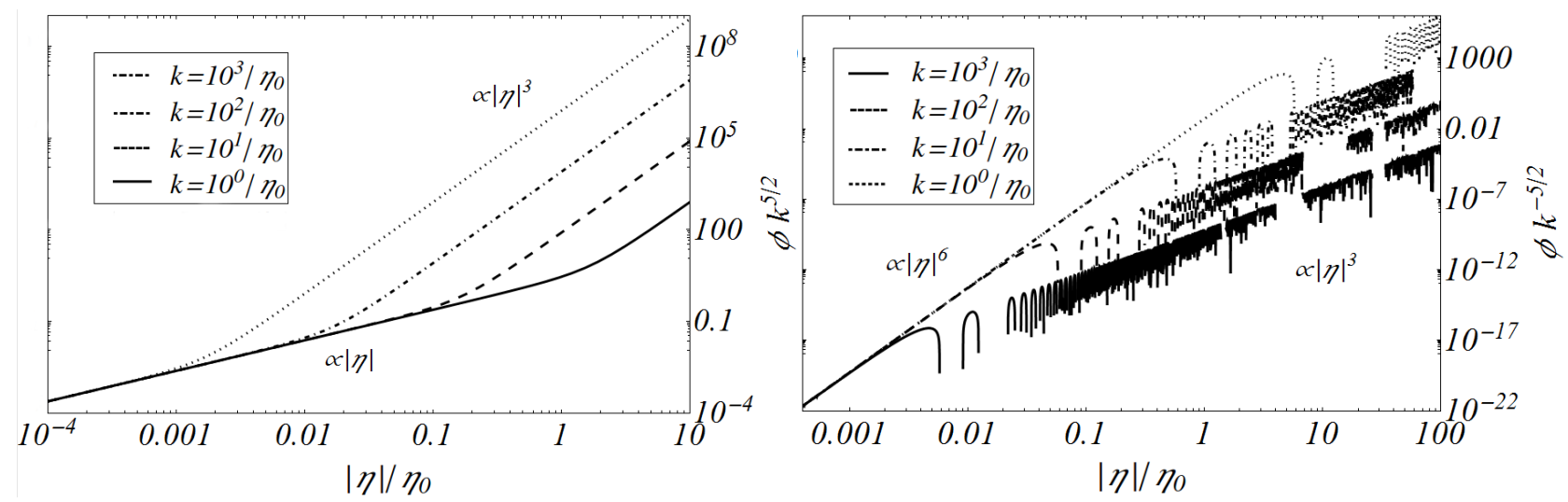

Figura 4.3: Evolução das perturbações escalares da métrica durante uma inflação exponencial. A evolução temporal das perturbações escalares da métrica, $\delta \phi(4.25 \mathrm{~b})$, apresenta oscilações com envoltórias proporcionais a potências 1, 2 e 3 do tempo conforme dentro do horizonte. Mas todos os modos apresentam a mesma amplitude ao sair do horizonte, onde começam a decair com a mesma amplitude. No lado esquerdo está representado o modo que decresce mais lentamente, sem oscilar. O lado direito corresponde aos modos oscilatórios que decaem rapidamente. As mudanças na evolução de cada curva ocorrem quando a escala em questão sai do horizonte de Hubble. 
Implementando perturbações escalares no tensor métrico, no calibre longitudinal (definido como $B=0$ e $\mu=0$ em (3.7)), em ausência de estresse anisotrópico $\sigma=0$ temos que $\phi=\psi$. Considerando a perturbação do campo escalar $\varphi(\eta) \rightarrow \varphi(\eta)+\delta \varphi(\eta, \mathbf{x})$ no tensor momento energia (4.15) obtemos:

$$
\begin{aligned}
T_{0}^{0} & =-\left(V+\frac{\dot{\varphi}^{2}}{2 a^{2}}-(\dot{\varphi})^{2} \frac{\phi}{a^{2}}+\dot{\varphi} \frac{\dot{\phi} \varphi}{a^{2}}+\delta \varphi V_{, \varphi}\right), \\
T_{0}^{i} & =\frac{i k_{i} \dot{\varphi} \delta \varphi}{a^{2}}, \\
T_{j}^{i} & =\delta_{i j}\left(-V+\frac{\dot{\varphi}^{2}}{2 a^{2}}-(\dot{\varphi})^{2} \frac{\phi}{a^{2}}+\dot{\varphi} \frac{\dot{\phi} \varphi}{a^{2}}-\delta \varphi V_{, \varphi}\right),
\end{aligned}
$$

e substituindo estas expressões na componente temporal da equação de conservação do tensor momento energia, no calibre longitudinal (definido como $B=0$ e $\mu=0$ em (3.7)), obtemos as seguintes equações

$$
\begin{gathered}
\ddot{\varphi}+2 \mathcal{H} \dot{\varphi}+a^{2} V_{, \varphi}=0, \\
\ddot{\delta \varphi}+2 \mathcal{H} \dot{\delta \varphi}+k^{2} \delta \varphi=-2 \phi a^{2} V_{, \varphi}-a^{2} \delta \varphi V_{, \varphi \varphi}+4 \dot{\phi} \dot{\varphi},
\end{gathered}
$$

até ordem zero e até primeira ordem nas perturbações, respetivamente. Adicionalmente, implementando as mesmas perturbações nas equações de Einstein obtemos a seguinte expressão para a evolução da perturbação métrica $\phi$ :

$$
-8 \pi G a^{2} \delta \varphi V_{, \varphi}=\ddot{\phi}+2 \phi \frac{\ddot{a}}{a}+6 \mathcal{H} \dot{\phi}+2 \mathcal{H}^{2} \phi+k^{2} \phi .
$$

Usando as condições de rolamento lento $(\epsilon \ll 1$ e $\lambda \ll 1)$ podemos obter as soluções às equações (4.24) durante uma inflação exponencial:

$$
\begin{gathered}
\varphi(\eta)=c_{3}+c_{4} \frac{\eta^{3}}{3} \\
\delta \varphi(\eta)=c_{5} k^{-3 / 2}(\sin \omega-\omega \cos \omega)+c_{6} k^{-3 / 2}(\cos \omega+\omega \sin \omega) \\
\phi(\eta)=c_{7} k^{-7 / 2} \omega\left(\omega^{2} \sin \omega+3 \omega \cos \omega-3 \sin \omega\right)+ \\
+c_{8} k^{-7 / 2} \omega\left(\omega^{2} \cos \omega-3 \omega \sin \omega-3 \cos \omega\right)
\end{gathered}
$$

que correspondem à densidade de energia do campo escalar sem perturbar $\varphi$, a perturbação do campo escalar $\delta \varphi$ (que é igual à solução para as perturbações tensoriais, ver Fig. (4.4)), e a perturbação métrica $\phi$ (ver Fig. (4.3)). Usamos a notação $\omega=k \eta$, e os diferentes $c_{i}$ representam as constantes de integração. A evolução da perturbação métrica apresenta somente termos decrescentes e o seu comportamento no fim da inflação pode ser aproximado como $\phi \rightarrow 3 c_{8} \eta k^{-5 / 2}$. 


\subsubsection{Valor esperado das perturbações e espectro primordial}

Assumindo que as perturbações no tensor momento energia possuem um carácter quântico, na aproximação de rolamento lento, a perturbação no campo escalar $\delta \varphi$ evolui de acordo com (4.24):

$$
\ddot{\delta} \varphi(k, \eta)+2 \mathcal{H} \delta \varphi(k, \eta)+k^{2} \delta \varphi(k, \eta)=0
$$

e pode ser reescrita como:

$$
\ddot{x}(k, \eta)+\left(k^{2}-\frac{\ddot{a}}{a}\right) x(k, \eta)=0,
$$

onde $x(k, \eta) \equiv a(\eta) \delta \varphi(k, \eta)$. Promovendo o campo $x(k, \eta)$ a um operador quântico:

$$
\hat{x}(k, \eta)=v(k, \eta) \hat{b}_{k}+v^{*}(k, \eta) \hat{b}_{k}^{+},
$$

onde os operadores criação de destruição, $\hat{b}_{k}^{+}$e $\hat{b}_{k}$, definem a quantização canônica mediante as suas regras de comutação

$$
\left[\hat{b}_{k}, \hat{b}_{k^{\prime}}^{+}\right]=\delta_{k k^{\prime}}, \quad\left[\hat{b}_{k}, \hat{b}_{k^{\prime}}\right]=\left[\hat{b}_{k}^{+}, \hat{b}_{k^{\prime}}^{+}\right]=0,
$$

enquanto que a função $v(k, \eta)$ representa a função de onda do sistema. Substituindo o operador (4.28) na equação de onda (4.27) obtemos que a função de onda $v(k, \eta)$ deve ser a solução à equação:

$$
\ddot{v}(k, \eta)+\left(k^{2}-\frac{\ddot{a}}{a}\right) v(k, \eta)=0 .
$$

Durante uma inflação exponencial (ver (2.55)) temos que $\ddot{a} / a=2 / \eta$, com o que a solução para a função de onda é dada por (4.25b):

$$
v(k, \eta)=C_{1} k^{-3 / 2}(\sin \omega-\omega \cos \omega)+C_{2} k^{-3 / 2}(\cos \omega+\omega \sin \omega),
$$

onde $C_{1}$ e $C_{2}$ são constantes de integração, e $\boldsymbol{\omega} \equiv k \eta$. Para que a função de onda seja quadraticamente normalizada debemos exigir que $C_{1}=i C_{2}$ (para que cancelem as oscilações no limite $k \eta \gg 1$ ), com o que a função de onda é simplificada para [Ma and Bertschinger, 1995]:

$$
v(k, \eta)=\frac{e^{-i k \eta}}{\sqrt{2 k}}\left(1-\frac{i}{k \eta}\right),
$$

com isto, o valor esperado no vácuo, do campo $x$ é:

$$
\begin{aligned}
\left\langle x^{*}(k, \eta) x\left(k^{\prime}, \eta\right)\right\rangle \equiv & \left\langle 0\left|x^{*}(k, \eta) x\left(k^{\prime}, \eta\right)\right| 0\right\rangle= \\
= & \left\langle 0\left|v^{*}(k, \eta) v\left(k^{\prime}, \eta\right) \hat{b}_{k}^{+} \hat{b}_{k^{\prime}}\right| 0\right\rangle+\left\langle 0\left|v^{*}(k, \eta) v^{*}\left(k^{\prime}, \eta\right) \hat{b}_{k}^{+} \hat{b}_{k^{\prime}}^{+}\right| 0\right\rangle+ \\
& +\left\langle 0\left|v(k, \eta) v\left(k^{\prime}, \eta\right) \hat{b}_{k} \hat{b}_{k^{\prime}}\right| 0\right\rangle+\left\langle 0\left|v(k, \eta) v^{*}\left(k^{\prime}, \eta\right) \hat{b}_{k} \hat{b}_{k^{\prime}}^{+}\right| 0\right\rangle= \\
= & v(k, \eta) v^{*}\left(k^{\prime}, \eta\right)\left\langle 0\left|\hat{b}_{k} \hat{b}_{k^{\prime}}^{+}\right| 0\right\rangle=v(k, \eta) v^{*}\left(k^{\prime}, \eta\right)\left\langle 0\left|\left[\hat{b}_{k}, \hat{b}_{k^{\prime}}^{+}\right]\right| 0\right\rangle= \\
= & v(k, \eta) v^{*}\left(k^{\prime}, \eta\right) \delta^{3}\left(k-k^{\prime}\right)=\frac{1}{2 k}\left(1+\frac{1}{(k \eta)^{2}}\right) \delta^{3}\left(k-k^{\prime}\right),
\end{aligned}
$$


desta forma, o valor esperado para as flutuações do campo escalar após sair do horizonte durante a inflação é

$$
\begin{aligned}
\left.P_{\delta \varphi}\right|_{k \ll \mathcal{H}} \delta^{3}\left(k-k^{\prime}\right) & \equiv \frac{\left\langle\delta \varphi^{*}(k, \eta) \delta \varphi\left(k^{\prime}, \eta\right)\right\rangle_{k \ll \mathcal{H}}}{(2 \pi)^{3}} \equiv \frac{\left\langle x^{*}(k, \eta) x\left(k^{\prime}, \eta\right)\right\rangle_{k \eta \ll 1}}{a^{2}}= \\
& =\frac{1}{2 a^{2} k} \delta^{3}\left(k-k^{\prime}\right)=\frac{H^{2}}{2 \mathcal{H}^{2} k} \delta^{3}\left(k-k^{\prime}\right),
\end{aligned}
$$

e o espectro de potências do campo escalar inflacionário no instante em que sai do horizonte é

$$
\left.\Delta_{\delta \varphi} \equiv \frac{k^{3}}{2 \pi^{2}} P_{\delta \varphi}\right|_{\mathcal{H}=k}=\left.\frac{H_{I}^{2}}{(2 \pi)^{2}}\right|_{\mathcal{H}=k},
$$

onde $H_{I}$ é o valor de $H$ durante a inflação exponencial. Se usarmos o invariante de calibre $\zeta$ (3.33):

$$
\zeta \equiv \Phi+\frac{\mathcal{H}}{k^{2}} \Theta=\phi+\frac{\mathcal{H}}{k^{2}} \theta-\frac{k^{2}}{6} \mu
$$

que no calibre espacialmente plano (definido como $\phi=\mu=0$ em (3.7)) se reduz a:

$$
\zeta=\frac{\mathcal{H}}{k^{2}} \theta=\frac{\mathcal{H}}{k^{2}} \frac{i k_{i} T_{i}^{0}}{\rho+\mathcal{P}}=-\frac{\mathcal{H}}{\dot{\varphi}} \delta \varphi
$$

podemos escrever o espectro de potência de $\zeta$, no instante em que sai do horizonte, como:

$$
\Delta_{\zeta}=\frac{\mathcal{H}^{2}}{\dot{\varphi}^{2}} \Delta_{\delta \varphi}=\frac{4 \pi G}{\epsilon} \Delta_{\delta \varphi},
$$

e graças a que $\dot{\zeta}=0$ fora do horizonte (ver equação (3.42)), o espectro da perturbação $\zeta$ vai ficar inalterado até entrar novamente no horizonte, durante o domínio da radiação (ou da matéria).

Durante o estágio de domínio da radiação e no calibre longitudinal (definido como $B=0$ e $\mu=0$ em (3.7)), podemos escrever

$$
\zeta=\phi+\frac{\mathcal{H}}{k^{2}} \theta_{\gamma} \approx \phi+\frac{\mathcal{H} \eta}{2} \phi=\frac{3}{2} \phi,
$$

onde usamos as condição inicial $\theta_{\gamma}=k^{2} \eta \psi / 2$ (3.51), a ausência de estresse anisotrópico $\sigma=0 \rightarrow$ $\phi=\psi(3.34 \mathrm{f})$, e o fato de que durante o período de domínio da radiação $a \propto \eta$, assim $\mathcal{H}=1 / \eta$. Finalmente, usando a componente 00 da equação de Einstein $((3.34)$ para $\dot{\phi} \approx 0)$ podemos escrever:

$$
\zeta=-\frac{3 \pi G a^{2} \rho_{\gamma}}{2 k^{2}} \delta_{\gamma}=-\frac{2 \pi G a^{2} \rho_{\gamma}}{k^{2}} \delta_{c} \approx-\frac{3 \mathcal{H}^{2}}{(2 k)^{2}} \delta_{c},
$$

onde usamos a condição inicial $\delta_{c}=3 \delta_{\gamma} / 4(3.49,3.54)$. Com todo isto, no instante em que os modos entram novamente no horizonte $\mathcal{H}=k$ obtemos:

$$
\zeta=-\frac{3}{4} \delta_{c}, \quad \operatorname{assim} \quad \Delta_{\zeta}=\frac{9}{16} \Delta_{c}=\frac{4 \pi G}{\epsilon} \Delta_{\delta \varphi},
$$

e o espectro inicial das perturbações na matéria escura fria finalmente pode ser escrito como:

$$
\Delta_{c}=\left.\frac{16 \pi G}{(3 \pi)^{2}} \frac{H_{I}^{2}}{\epsilon}\right|_{\mathcal{H}=k},
$$


ficando expresso em função do parâmetro de rolamento lento $\epsilon$ e do parâmetro de Hubble durante a inflação $H_{I}$. Estes dois parâmetros são constantes em primeira aproximação, pelo que o espectro inicial $\Delta_{c}$ é aproximadamente independente da escala $k$, mas possui uma pequena variação parametrizada no conhecido índice espectral escalar $n_{s}$ (e avaliada usualmente na escala $k_{*} \equiv 0.05 \mathrm{Mpc}$ ) que é definido como:

$$
n_{s}-1 \equiv \frac{\partial \ln \Delta_{c}}{\partial \ln k}=\frac{\partial \ln \Delta_{c}}{\partial \ln \mathcal{H}}=\frac{\mathcal{H}}{\dot{\mathcal{H}}}\left(2 \frac{\dot{H}}{H}-\frac{\dot{\epsilon}}{\epsilon}\right)=-3 \epsilon-\lambda,
$$

até primeira ordem em $\epsilon$ e $\lambda$, e onde avaliamos todas as grandezas no instante em que cada escala cruza o horizonte $\partial_{k}=\partial_{\mathcal{H}}=\dot{\mathcal{H}}^{-1} \partial \eta$, também como a definição dos parâmetros de rolamento lento (4.13). Com isto, o espectro primordial pode ser parametrizado ao redor da escala $k_{*}$ como:

$$
\Delta_{c}(k)=\left.\frac{16 \pi G}{(3 \pi)^{2}} \frac{H_{I}^{2}}{\epsilon}\right|_{\mathcal{H}=k_{*}}\left(\frac{k}{k_{*}}\right)^{n_{s}-1}, \quad n_{s}=1-3 \epsilon-\lambda
$$

\subsection{EVoluÇão Das PerturbaÇÕes tensoriais Durante a inflaÇão}
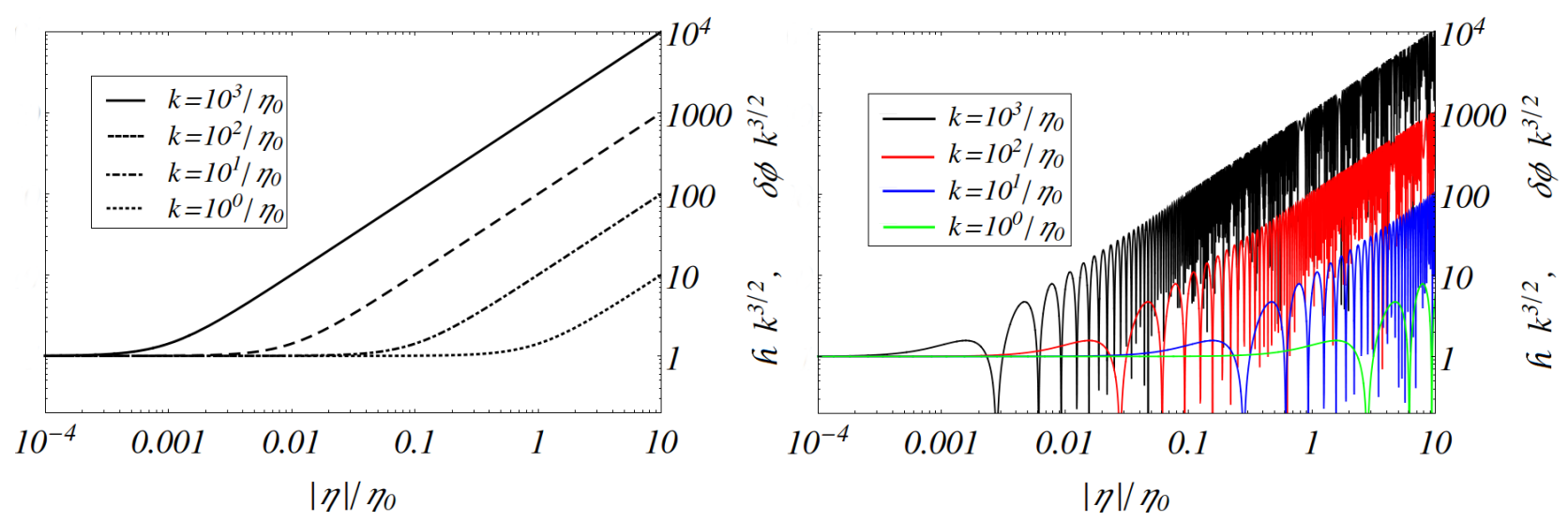

Figura 4.4: Evolução das perturbações tensoriais e escalares durante uma inflação exponencial.

Se mostra a evolução temporal das perturbações tensoriais $\hbar$ (4.47), e escalares $\delta \varphi(4.25 \mathrm{~b})$, já que as duas obedecem a mesma equação. Do lado direito se apresenta uma solução geral, onde a amplitude das ondas gravitacionais oscila enquanto o universo expande. Do lado esquerdo, uma solução particular, onde uma escolha particular das constantes de integração fazem com que os termos oscilatórios se cancelem, o que faz que a amplitude das ondas gravitacionais seja agora a envoltória da solução geral oscilatória. Para qualquer escolha das constantes de integração o espectro primordial das ondas gravitacionais (no fim do período acelerado $\eta \rightarrow 0^{-}$) é predito plano para todas as escalas que conseguiram sair do horizonte durante a inflação, já que a amplitude de todos os modos que saem do horizonte vai para o mesmo valor constante multiplicado por $k^{-3 / 2}$.

Durante o período inflacionário gerado por um fluido tal que $\mathcal{P}=-\rho$ obtemos que $a \propto-\frac{1}{\eta}(2.56)$, onde $\eta \in(-\infty, 0)$, como solução às equações de Einstein não perturbadas:

$$
8 \pi G a^{2} \bar{\rho}=3 \mathcal{H}^{2}, \quad \quad 8 \pi G a^{2} \overline{\mathcal{P}}=-2 \mathcal{H}-\mathcal{H}^{2}, \quad \rightarrow \quad \frac{\ddot{a}}{a}+2 \mathcal{H}^{2}=0 .
$$


Usando este último resultado dentro da equação de evolução das perturbações tensoriais (3.75) sem fontes, se obtém a seguinte equação para a evolução das ondas gravitacionais durante o período inflacionário:

$$
\ddot{h}+2 \mathcal{H} \dot{h}+k^{2} h=0,
$$

que tem como solução geral

$$
h(\eta)=c_{1} k^{-3 / 2}(\sin \omega-\omega \cos \omega)+c_{2} k^{-3 / 2}(\cos \omega+\omega \sin \omega)
$$

onde $\mathfrak{\omega} \equiv k \eta$, e, $c_{1}$ e $c_{2}$ são constantes de integração (ver Fig. 4.4). Com isto, no caso em que $\eta \rightarrow 0^{-}$ (fim da inflação) obtemos o seguinte limite para o espectro de potências das perturbações tensoriais:

$$
\hbar^{*} \hbar \rightarrow \frac{\left(c_{2}\right)^{2}}{k^{3}}, \quad \Delta_{h} \equiv\left\langle\hbar^{*} \hbar\right\rangle \frac{k^{3}}{2 \pi^{2}}=\frac{\left(c_{2}\right)^{2}}{2 \pi^{2}} .
$$

$\mathrm{O}$ valor de $c_{2}$ pode ser obtido assumindo novamente (como se fez no casso das perturbações do campo escalar) que as perturbações primordiais possuem um carácter quântico (sessão (4.2.2)). Fazendo a mudança de variável $\hbar \equiv \sqrt{16 \pi G} x / a$ [Dodelson, 2003], a equação (4.46) pode ser escrita na forma (4.27), e cuja solução produz o valor esperado (4.33):

$$
\left\langle x^{*}(k, \eta) x\left(k^{\prime}, \eta\right)\right\rangle=\frac{1}{2 k}\left(1+\frac{1}{(k \eta)^{2}}\right) \delta^{3}\left(k-k^{\prime}\right) .
$$

Desta forma o espectro de potências das ondas gravitacionais fora do horizonte (quando $k \eta \ll 1$ ) fica espresso como:

$$
\begin{aligned}
\left.P_{h}\right|_{k \ll \mathcal{H}} \delta^{3}\left(k-k^{\prime}\right) & \equiv \frac{\left\langle\hbar^{*}(k, \eta) h\left(k^{\prime}, \eta\right)\right\rangle_{k \ll \mathcal{H}}}{(2 \pi)^{3}}=\frac{16 \pi G}{a^{2}}\left\langle x^{*}(k \eta) x\left(k^{\prime}, \eta\right)\right\rangle_{k \eta \ll 1}= \\
& =\frac{8 \pi G}{a^{2} k} \delta^{3}\left(k-k^{\prime}\right)=\frac{8 \pi G H^{2}}{\mathcal{H}^{2} k} \delta^{3}\left(k-k^{\prime}\right),
\end{aligned}
$$

e avaliado no instante em que cada escala sai do horizonte gera a seguinte expressão (levando agora em conta um fator 2 devido aos modos independentes $h_{+}$e $h_{\times}$)

$$
P_{h}=\left.16 \pi G \frac{H_{I}^{2}}{k^{3}}\right|_{\mathcal{H}=k},
$$

e possue a variação:

$$
n_{T} \equiv \frac{\partial \ln P_{h}}{\partial \ln k}+3=\frac{\partial \ln H^{2}}{\partial \ln \mathcal{H}}=2 \frac{\mathcal{H}}{\dot{\mathcal{H}}} \frac{\dot{H}}{H}=-2 \frac{\epsilon}{1-\epsilon}=-2 \epsilon,
$$

até primeira ordem em $\epsilon$ (4.13), com o que podemos escrever o espectro das ondas gravitacionais ao redor da escala $k_{*}$ como sendo

$$
P_{h}(k)=\left.P_{h}\right|_{\mathcal{H}=k_{*}}\left(\frac{k}{k_{*}}\right)^{n_{T}-3}=\left.16 \pi G \frac{H_{I}^{2}}{k_{*}^{3}}\right|_{\mathcal{H}=k_{*}}\left(\frac{k_{*}}{k}\right)^{3+2 \epsilon}
$$




\subsection{NECESSIDADE DE REAQUECIMENTO APÓS A INFLAÇÃO}

A inflação, como formulada anteriormente, sofre de vários problemas. Um deles ocorre devido ao super-esfriamento da radiação causado pela rápida expansão do universo durante o período de expansão acelerada, já que quando ela é produzida por um campo que não interage com a radiação, sua densidade de energia decresce como $\rho_{r} \propto a^{-4}$ (ver equação (2.37)). Agora, para que a inflação resolva o problema do horizonte o fator de escala do universo deve crescer pelo menos 28 ordens de grandeza (ver equação (4.12)) durante o período primordial acelerado.

Com isso, se a radiação evolui livremente durante esse período a sua densidade de energia, e consequentemente a sua temperatura, vão decrescer respectivamente em 112 e 28 ordens de grandeza. Dada a escala de energia da grande unificação $10^{16} \mathrm{GeV}=10^{25} \mathrm{eV} \sim \mathcal{O}\left(10^{21}\right) \mathrm{K}$ (que é escala de energia efetiva do período inflacionário) e sabendo que o campo inflacionário dominava o universo já no começo da inflação, obtemos automaticamente que a temperatura da radiação no começo da inflação não pode ser maior que $\sim 10^{21} \mathrm{~K}$ e portanto, no fim da inflação vai ser da ordem de $\sim 10^{-7} \mathrm{~K}$ (menor que a temperatura observada hoje $\sim 10^{-4} \mathrm{~K}$ ).

Então, é preciso que haja algum mecanismo que reaqueça o universo após o período inflacionário. Uma forma de ser fazer isto é modificar o potencial do campo inflacionário, de tal forma que possua um mínimo profundo e um termo dissipativo logo após o fim da inflação. Deste modo o campo escalar adquire considerável energia cinética que pode ser transferida para a radiação aquecendo o universo. Esto introduz um problema de ajuste fino nos parâmetros livres do potencial do campo inflacionário, que deve ser muito plano durante o período inflacionário mas que deve possuir uma queda abrupta, no fim deste período, capaz de elevar a temperatura da radiação em quase os 28 ordens de grandeza que esta diminuiu durante o período inflacionário. 


\section{DENSIDADE DO VÁCUO VARIÁVEL}

Neste capítulo estudaremos as consequências gerais de uma densidade do vácuo que varia monotonamente com o parâmetro de Hubble, com o fim de caracterizar a dependência mais simples capaz de reproduzir universos com eras de Sitter inicial e final. Também apresentaremos argumentos fenomenológicos que levaram a este tipo de propostas, enquanto se tentava, principalmente, atenuar os problemas de coincidência e da constante cosmológica presentes no modelo padrão $\Lambda$ CDM.

Finalmente apresentaremos motivações mais fundamentais para tais propostas. Baseados em uma das principais predições do formalismo do grupo de renormalização (que surge nas teorias de calibre renormalizáveis que constituem a base do modelo padrão da física de partículas) isto é, a variação das cargas e parâmetros de acoplamento com a escala de energia, como consequência da dependência das funções de Green com a escala de energia introduzida pelos diferentes esquemas de renormalização.

\subsection{UNIVERSO EM EXPANSÃo COM UMA DENSIDAde DO VÁCUO QUE DECRESCE MONO- TONAMENTE}

Nesta seção estudaremos o comportamento geral do parâmetro de Hubble para um universo plano composto por um fluido material e uma densidade do vácuo, que por sua vez possui um termo de constante cosmológica e um termo proporcional a uma potência positiva do parâmetro de Hubble. Na verdade a análise a seguir funciona ainda em casos mais gerais, sempre que a densidade do vácuo $\Lambda$ for menor que $3 H^{2}$ para um intervalo conexo e aberto de $H$ (exigido pela condição fraca de energia da componente material $8 \pi G \rho=3 H^{2}-\Lambda>0$ ) e igual a $3 H^{2}$ na fronteira daquele intervalo.

Obteremos como conclusão que algumas dependências da densidade do vácuo que crescem monotonamente com o parâmetro de Hubble impedem a existência de singularidades em processos puramente gravitacionais, gerando universos com eras de Sitter inicial e final.

As equações de Einstein (2.44) para um universo plano em expansão, perfeitamente isotrópico e homogêneo, que contém um fluido ideal com equação de estado $\mathcal{P}(t)=\omega \rho(t)$ para um parâmetro constante $\omega>0$, e uma densidade do vácuo com equação de estado $\mathcal{P}_{\Lambda}(t)=-\rho_{\Lambda}(t) \equiv-\Lambda(t) / 8 \pi G$, podem ser escritas em função do tempo cósmico como:

$$
8 \pi G \rho+\Lambda=3 H^{2}, \quad 8 \pi G \omega \rho-\Lambda=-2 \frac{d H}{d t}-3 H^{2} .
$$


Substituindo a densidade de energia do fluido material de uma equação na outra, obtemos uma equação diferencial para o parâmetro de Hubble em função da densidade do vácuo:

$$
2 \frac{d H}{d t}+(1+\omega)\left(3 H^{2}-\Lambda\right)=0
$$

ou

$$
\frac{2}{a} \frac{d^{2} a}{d t^{2}}+H^{2}(1+3 \omega)=\Lambda(1+\omega)
$$

Neste cenário obtemos que:

$$
\frac{d^{2} a}{d t^{2}}=0, \quad \text { quando } \quad H^{2}=\frac{1+\omega}{1+3 \omega} \Lambda
$$

como também

$$
\frac{d H}{d t}=0, \quad \text { quando } \quad H^{2}=\frac{1}{3} \Lambda,
$$

onde $(1+\omega) /(1+3 \omega)>1 / 3$ para todo $\omega$ (mas estamos interessados apenas no caso $\omega \geqslant 0$ ).

\subsubsection{Universos com eras de Sitter inicial e final}

No caso em que $\Lambda(H)$ cresce mais lentamente que $3 H^{2}$ para valores de $H<H_{\star}$; enquanto que cresce mais rapidamente que $3 H^{2}$ quando $H>H_{\star}$, sempre que $\Lambda\left(H_{\star}\right)<3 H_{\star}^{2}$, exigido pela condição fraca de energia do fluido material $\left(8 \pi G \rho=3 H^{2}-\Lambda>0\right)$; obtemos que a equação diferencial (5.2a) possui dois pontos onde $d H / d t=0$, que definimos como $3 H_{-}^{2} \equiv \Lambda\left(H_{-}\right)$e $3 H_{+}^{2} \equiv \Lambda\left(H_{+}\right)$, tais que $H_{-}<H_{\star}<H_{+}$ (ver figura 5.1).

Neste caso, a condição fraca de energia para o fluido material faz com que a solução da equação (5.2a) para o parâmetro de Hubble esteja demarcada por $H \in\left(H_{-}, H_{+}\right)$, o que impede a existência de estados singulares, já que $\rho_{T}=3 H^{2} / 8 \pi G$ deve permanecer finito sempre.

Adicionalmente, devido a que $d H / d t \rightarrow 0$ quando $H \rightarrow H_{-}$pela direita, e quando $H \rightarrow H_{+}$pela esquerda, obtemos que $H$ somente pode tender assintoticamente aos valores $H_{ \pm}$quando $t \rightarrow \mp \infty$, respetivamente. Com isto obtemos necessariamente épocas inicial e final assintoticamente de Sitter.

Se assumirmos a seguinte expressão para a dependência temporal da densidade do vácuo:

$$
\Lambda(H)=c_{0}+c_{\alpha} H^{\alpha}, \quad \operatorname{com} \quad \alpha>2,
$$

podemos encontrar 3 casos diferentes de interesse físico, onde o primeiro acaba sendo o mais interessante, já que possui eras aceleradas inicial e final com uma era desacelerada no período intermediário:

1. No caso em que a equação (5.3) possui duas soluções diferentes $H_{1}$ y $H_{2}$ com $H_{1}<H_{2}$ (ver figura 5.1). Devido a que $(1+\omega) /(1+3 \omega)>1 / 3$ para todo $\omega$, obtemos que a equação (5.4) também possui duas soluções diferentes que denotamos por $H_{-}$e $H_{+}$com $H_{-}<H_{+}$(ver Figura 5.1). 
Usando a primeira equação de Einstein (5.1) e impondo a positividade da densidade de energia do fluido material obtemos que o parâmetro de Hubble $H^{2} \notin\left[0, H_{-}^{2}\right) \cup\left(H_{+}^{2}, \infty\right)$, ficando restrito a um intervalo finito e conexo (se $\Lambda(H)$ apresenta um crescimento monótono). Como também obtemos que $d^{2} a / d t^{2}<0$ no intervalo $H^{2} \in\left(H_{1}^{2}, H_{2}^{2}\right)$, igual a zero na fronteira deste intervalo, e maior que zero fora dele. Em particular $d^{2} a / d t^{2} \rightarrow H^{2} a$ quando $H^{2} \rightarrow H_{ \pm}^{2}$.

\section{Conclusão:}

No caso de um universo em expansão o fator de escala cresce aceleradamente enquanto $H$ decresce ao longo do intervalo $\left(H_{2}, H_{+}\right)$(sendo equivalente ao período inflacionário do modelo cosmológico padrão); passa logo a se expandir desaceleradamente durante o intervalo de tempo em que $H$ continua decrescendo dentro do intervalo $\left(H_{1}, H_{2}\right)$ (se este período for dominado não por um único fluido material, mas sim pela mistura de dois deles -tais como a radiação e a matéria escuraestaríamos reproduzindo as condições observadas do nosso universo); e finalmente passa a se expandir aceleradamente enquanto o fator de Hubble decresce dentro do intervalo $\left(H_{-}, H_{1}\right)$ (que equivale ao período de aceleração atual no modelo $\Lambda \mathrm{CDM})$.

Adicionalmente, devido a que $d H / d t \rightarrow 0$ quando $H \rightarrow H_{ \pm}$obtemos que as eras aceleradas inicial e final são assintoticamente de Sitter nos limites $t \rightarrow \mp \infty$, respetivamente, gerando um universo arbitrariamente velho e acelerado inicialmente, o que resolve o problema do horizonte.

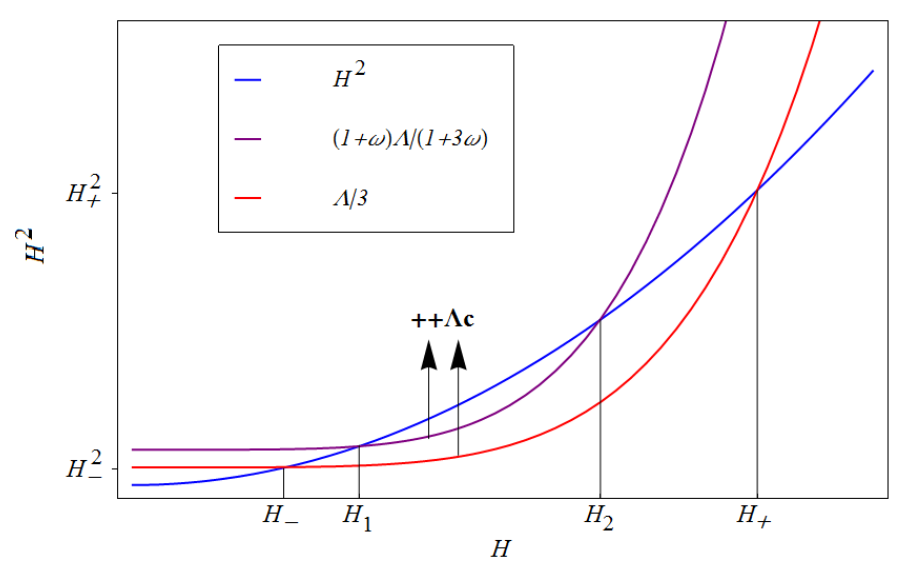

Figura 5.1: Universos com eras aceleradas inicial e final, e um estágio intermediário desacelerado. No caso em que a densidade do vácuo possui um termo que cresce mais rapidamente que $3 H^{2}$ e a equação $d H / d t=0$ possui duas soluções $\left\{H_{-}, H_{+}\right\}$, o parâmetro de Hubble fica demarcado por estes valores. Se adicionalmente a equação $d^{2} a / d t^{2}=0$ possui duas soluções $\left\{H_{1}, H_{2}\right\}$, obtemos que a expansão acontece aceleradamente nos intervalos abertos $\left(H_{-}, H_{1}\right)$ e $\left(H_{2}, H_{+}\right)$, enquanto que acontece desaceleradamente no intervalo $\left(H_{1}, H_{2}\right)$.

2. Caso em que a equação (5.3) possui uma única solução $H^{\prime}$ (ver figura 5.2). Novamente, devido a que $(1+\omega) /(1+3 \omega)>1 / 3$ para todo $\omega$, temos que a equação (5.4) possui duas soluções diferentes que denotamos como $H_{-}$e $H_{+}$, com $H_{-}<H_{+}$. Neste caso, o universo se expande sempre aceleradamente exceto quando $H=H^{\prime}$, onde $d^{2} a / d t^{2}=0$.

Como no caso anterior obtemos que $\rho \rightarrow 0$, também como $H$ tende a seus valores limite $H_{ \pm}$, enquanto que $\Lambda$ tende a $3 H_{ \pm}^{2}$ quanto $t \rightarrow \mp \infty$; gerando um universo livre do problema do 
horizonte, mas sempre acelerado (exceto no instante em que $H=H^{\prime}$ ), o que impede a existência dos períodos de domínio da radiação de da matéria escura.

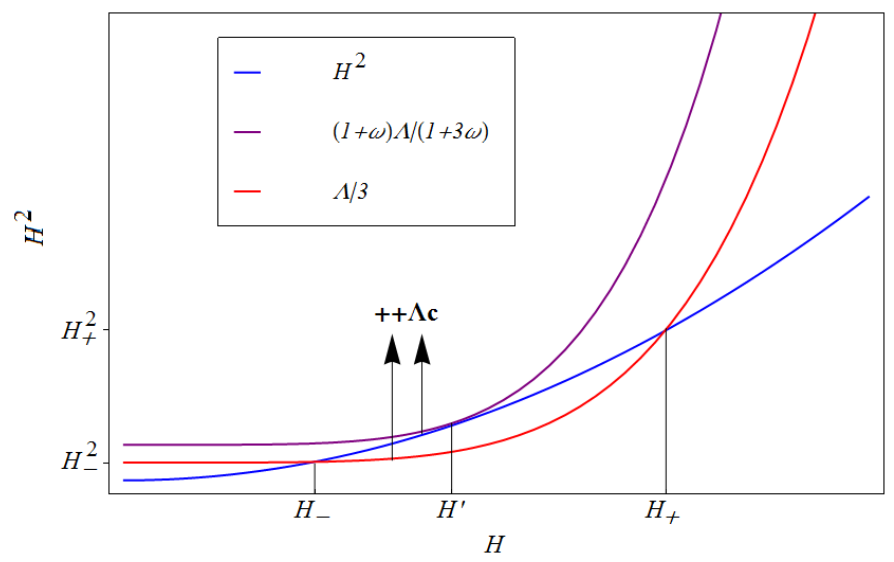

Figura 5.2: Universos acelerados exceto em um instante de tempo intermediário.

No caso em que a densidade do vácuo possui um termo que cresce mais rapidamente que $3 H^{2}$ e a equação $d H / d t=0$ possui duas soluções $\left\{H_{-}, H_{+}\right\}$, o parâmetro de Hubble fica demarcado por estes valores. Se adicionalmente a equação $d^{2} a / d t^{2}=0$ possui uma solução única $H^{\prime}$, obtemos que a expansão acontece aceleradamente sempre, exceto no instante em que $H=H^{\prime}$.

3. Quando a equação (5.3) não possui soluções reais, mas devido a que $(1+\omega) /(1+3 \omega)>1 / 3$ a equação (5.4) possui ainda duas soluções diferentes $H_{-}$e $H_{+}$com $H_{-}<H_{+}$(ver figura 5.3), obtemos que o universo sempre se expande aceleradamente enquanto o parâmetro de Hubble decresce assintoticamente do valor $H_{+}$até o valor $H_{-}$.

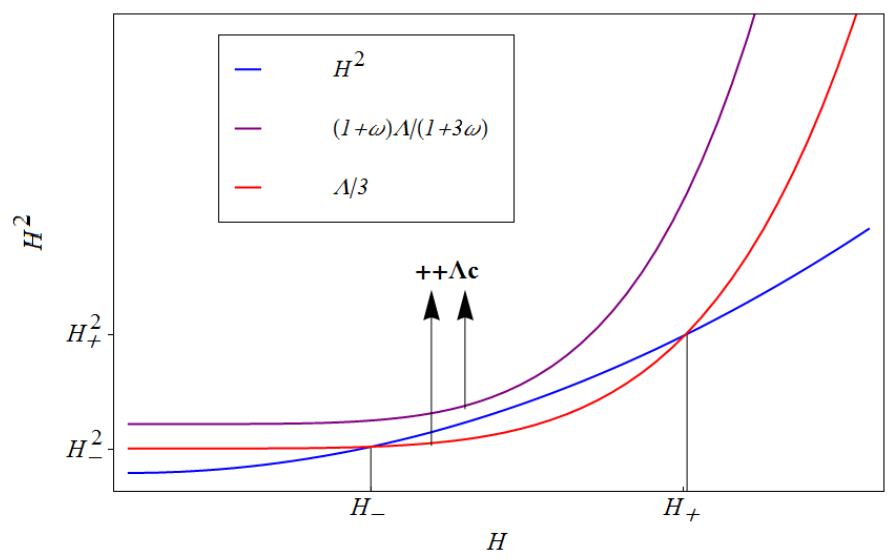

Figura 5.3: Universos que se expandem sempre aceleradamente.

No caso em que a densidade do vácuo possui um termo que cresce mais rapidamente que $3 H^{2}$ e a equação $d H / d t=0$ possui duas soluções $\left\{H_{-}, H_{+}\right\}$, o parâmetro de Hubble fica demarcado por estes valores. Se adicionalmente a equação $d^{2} a / d t^{2}=0$ não possui soluções reais, obtemos que a expansão acontece sempre de forma acelerada.

Nos casos em que a equação (5.3) possui uma solução, ou não possui soluções reais, a partir da primeira equação de Einstein temos que $\rho<0$, e portanto não são casos de interesse físico.

O primeiro caso é mais interessante fisicamente, nele o parâmetro de Hubble está demarcado entre dois valores finitos e positivos, impedindo qualquer estado singular, começado com um período de expansão 
acelerado dominado por uma densidade do vácuo que decresce de forma lenta (recreando o período inflacionário), seguido por um período desacelerado dominado pela densidade de energia material $\rho$ (que deve tratar-se no caso mais realístico de uma mistura de matéria escura, bárions, fótons, e neutrinos), para logo voltar a um estado dominado pela densidade do vácuo que tende a um valor constante produzindo novamente um período de expansão acelerada assintoticamente de Sitter.

\subsubsection{Universos em que $3 H^{2}$ cresce mais rápido do que a densidade do vácuo $\Lambda$}

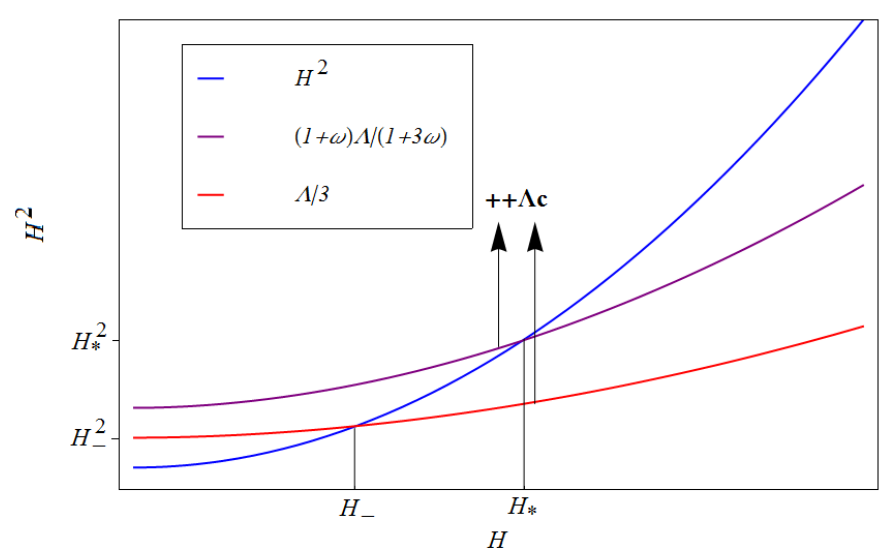

Figura 5.4: Universos com uma era inicial desacelerada e uma era final acelerada.

No caso em que a densidade do vácuo cresce mais lentamente que $3 H^{2}$ e a equação $d H / d t=0$ possui uma solução única $H_{-}$, e o parâmetro de Hubble fica demarcado inferiormente por este valor. Neste casso, também a equação $d^{2} a / d t^{2}=0$ possui uma solução única $H_{*}$ e obtemos que a expansão acontece desaceleradamente no intervalo aberto $\left(H_{*}, \infty\right)$, enquanto que acontece aceleradamente no intervalo $\left(H_{-}, H_{*}\right)$. Lembre-se que o tempo flui de direita para esquerda para universos espacialmente planos, devido às equações de Einstein, se exigimos a validade da condição de energia fraca.

Neste caso, tanto a equação (5.3) como a equação (5.4) possuem soluções únicas $H_{-}$e $H *$ respetivamente, com $H_{-}>H *$, ver figura 5.4. Sobre tais condições o universo em expansão possui uma singularidade inicial, partindo de $H \rightarrow \infty$ (onde $\rho=3 H^{2} / 8 \pi G \rightarrow \infty$ ) e se expandindo de forma desacelerada até chegar no valor $H=H_{*}$. A partir deste ponto o universo entra em um período de expansão acelerada, onde o parâmetro de Hubble tende assintoticamente ao valor terminal $H \rightarrow H_{-}$quando $t \rightarrow \infty$, já que $d H / d t \rightarrow 0$ neste limite, enquanto $\rho \rightarrow 0$. Como nos casos anteriores, a partir da análise da primeira equação de Einstein temos que os valores $H<H_{-}$são proibidos se exigirmos que $\rho>0$.

Este comportamento não difere qualitativamente do comportamento do modelo padrão $\Lambda \mathrm{CDM}$, formado por um período inicial de expansão desacelerada dominada por fluidos com pressão positiva (que extrapolado até o passado conduz à formação de uma singularidade inicial, gerando o problema do horizonte) e seguidos de um período final acelerado, onde o universo é dominado por uma densidade do vácuo que tende a um valor constante.

Um caso particularmente simples desta família de modelos pode ser parametrizado como:

$$
\Lambda(t)=c_{0}+c_{\alpha} H^{\alpha}, \quad \operatorname{com} \quad \alpha<2 .
$$




\section{1 .3 Resumo}

Se a densidade do vácuo cresce monotonamente com o parâmetro de Hubble da forma $\Lambda(t)=c_{0}+c_{\alpha} H^{\alpha}$, e $\operatorname{com} \alpha<2$, obtemos que um universo plano possui um período inicial de expansão desacelerada (devido ao domínio dos fluidos materiais) a partir de um estado singular, mas com um período final de expansão acelerada, dominado pela densidade do vácuo que tende a um valor constante $H \rightarrow \sqrt{c_{0} / 3}=$ cte . Este tipo de sistema se comporta qualitativamente da mesma forma que o modelo inflação $+\Lambda C D M$. O resultado análogo para um sistema que colapsa gravitacionalmente resulta na formação de uma singularidade devido a que o estágio final é sempre um colapso acelerado com uma densidade total que cresce monotonamente, e sem limite, atingindo um estado singular.

No caso em que $\alpha>2$ e a equação $d H / d t=0$ possui duas soluções reais finitas, obtemos que o parâmetro de Hubble permanece demarcado pelas duas soluções, impedindo a existência de estados singulares, já que $\rho_{T}=3 H^{2} / 8 \pi G$. Em tais casos, o universo começa com um período acelerado dominado pelo vácuo (com um estado inicial não singular onde $H \approx\left(3 / c_{\alpha}\right)^{1 /(\alpha-2)}$ ) passando ou não por um período desacelerado (se a equação $d^{2} a / d t^{2}=0$ possui ou não duas soluções reais) dominado pelos fluidos materiais, e acabando com um período de expansão acelerada e novamente dominado pelo vácuo enquanto $H \rightarrow \sqrt{c_{0} / 3}=$ cte..

O caso de maior interesse físico é aquele em que esteja presente um período intermediário de expansão desacelerada, já que reproduz qualitativamente em ordem cronológica o período inflacionário, os períodos dominados pela radiação e pela matéria, e o período atual acelerado. Analogamente ao caso do universo em expansão, um sistema que colapsa gravitacionalmente enfrentará um estado final desacelerado sem a formação de uma singularidade, já que tanto a densidade total atinge assintoticamente um valore constante, enquanto a densidade de energia do fluido material decresce gradualmente.

\subsection{Proposta fenomenológica para a forma de $\Lambda(t)$}

O objetivo principal desta seção é propor uma classe de modelo de energia escura como sendo um vácuo dinâmico com uma dependência explícita no parâmetro de Hubble $\Lambda=\Lambda(H)$, que por sua vez depende do tempo $H=H(t)$. Como veremos, esta dinâmica está em harmonia com o esperado pela teoria quântica de campos, que prevê uma variação no valor esperado do vácuo com a escala de energia do sistema, no entanto nossa escolha para a dependência explícita da função $\Lambda(H)$ nesta seção vai ser de fato fenomenológica.

A presença de duas componentes relevantes em um dado instante de tempo no universo (a densidade do vácuo $\rho_{\Lambda}$ mais a densidade de energia do fluido material dominante $\rho$ ) introduz naturalmente a seguinte razão:

$$
\beta(t)=\frac{\rho_{\Lambda}-\rho_{\Lambda}^{\infty}}{\rho+\rho_{\Lambda}}
$$

onde $\rho_{\Lambda}^{\infty}$ e a parte constante da densidade efetiva do vácuo. Com isto, o parâmetro $\beta(t)$ quantifica a variação na densidade do vácuo comparada com a densidade total do universo. Este parâmetro possui as seguintes propriedades de interesse físico:

(i) Se $\rho_{\Lambda}=\rho_{\Lambda}^{\infty}$, então $\beta=0$, e o modelo torna-se o modelo $\Lambda$ CDM. 
(ii) Se $\rho_{\Lambda}^{\infty}=0$, então a razão (5.7) define a fração da densidade do vácuo no universo. Adicionalmente, se esta fração permanece constante ao longo da evolução cósmica obtemos que:

$$
\rho_{\Lambda}(t)=\beta \rho_{T}(t), \quad \text { ou } \quad \Lambda=3 \beta H^{2}(t)
$$

Este tipo de modelo foi bem estudado por [Freese et al., 1987; Arcuri and Waga, 1994]. No entanto o modelo não se ajusta bem às observações cosmológicas [Basilakos et al., 2009]. A principal dificuldade deste tipo de modelos é a inexistência de uma transição de um período desacelerado para o recente período de expansão acelerada, o que é observado nos dados provenientes de supernovas. Os modelos do tipo (5.8) implicam que o universo é sempre acelerado ou sempre desacelerado dependendo do valor do parâmetro constante $\beta$.

(iii) Se em vez disto, consideramos a razão (5.7) como sendo constante, mas com $\rho_{\Lambda}^{\infty} \neq 0$ obtemos que

$$
\Lambda(t)=c_{0}+3 \beta H^{2}(t)
$$

onde $c_{0}=8 \pi G \rho_{c r}^{0}\left(\Omega_{\Lambda}^{0}-\beta\right)$. Esse modelo tem sido bastante estudado na literatura [Shapiro et al., 2003; Basilakos et al., 2009, 2012], e podem ser obtidos como casso particular do modelo proposto por Waga [1993]. Diferente do modelo (5.8), a presença de um termo constante na densidade do vácuo permite a transição de um universo desacelerado para um universo acelerado; e além disso é equivalente ao modelo $\Lambda \mathrm{CDM}$ no limite de $\beta \rightarrow 0$. Note-se que a o modelo (5.8) não possui um caso limite equivalente ao modelo $\Lambda$ CDM.

Em geral a razão (5.7) pode não permanecer constante durante a evolução do universo. Neste caso a energia do vácuo assume a forma geral:

$$
\rho_{\Lambda}(t)=c_{0} / 8 \pi G+\beta(t) \rho_{\mathrm{T}}(t), \quad \text { ou } \quad \Lambda(t)=c_{0}+3 \beta(t) H^{2} .
$$

Agora que $\beta(t)$ depende do tempo, o valor atual para a densidade do vácuo é $\Lambda_{0}=c_{0}+3 \beta\left(t_{0}\right) H_{0}^{2}$, onde $t_{0}$ é o tempo cósmico hoje. Supondo que podemos expandir o parâmetro $\beta(t)$ como:

$$
\beta(t)=v+\alpha\left(H / H_{I}\right)^{n},
$$

onde $v, \alpha$ e $H_{I}$ são constantes cuja interpretação será dada na seguinte seção, e $n$ é um inteiro positivo $n \geqslant 1$. Muitos aspectos do caso $n=1$ para uma geometria espacialmente plana foram estudados em [Lima and Maia, 1994]. Posteriormente, estes autores discutiram cenários com geometrias abertas e fechadas [Lima and Trodden, 1996].

Usando este ansatz geral para $\beta(t)$ podemos reescrever a densidade do vácuo como:

$$
\Lambda(H)=c_{0}+3 v H^{2}+3 \alpha \frac{H^{2+n}}{H_{I}^{n}} .
$$

Sem fazer considerações adicionais, o inteiro $n$, na equação (5.12), pode ser qualquer $n \geqslant 1$. Obviamente o caso $n=0$ não é considerado porque corresponde à situação $\beta=v+\alpha=$ cte, considerada na formulação original. Modelos não triviais requerem que $n \geqslant 1$. Em contraste, o caso com $\alpha=0$ leva 
ao modelo (5.9), que é adequado para modelar o universo recente [España-Bonet et al., 2004; Grande et al., 2011; Shapiro et al., 2003; Basilakos et al., 2009] mas não para os estágios primordiais.

O termo constante $c_{0}$ em (5.12) obviamente representa a contribuição dominante para baixas energias (quando $H \approx \mathcal{O}\left(H_{0}\right) \ll H_{I}$ se $v \ll 1$ ). O termo $H^{2}$ representa uma pequena correção (se $v \ll 1$ ) ao termo dominante hoje, enquanto que dirige a evolução da energia do vácuo durante os estágios intermediários, quando os fluidos materiais dominam a evolução do universo. Por outro lado, as potências $H^{2+n}(n \geqslant 1)$ adquirem grande relevância durante os estágios primitivos do universo, próximo da escala de energia associada a $H_{I}$, interpretada neste modelo como a taxa de expansão durante a inflação.

Devido a que $H_{I}$ é usualmente assumido como sendo muito maior que $H_{0}$, é claro que $\beta\left(t_{0}\right) \simeq v \mathrm{e}$ independe do valor de $n$ para baixas energias, portanto o valor da energia do vácuo hoje é essencialmente $\Lambda_{0}=c_{0}+3 v H_{0}^{2}$ para todos os modelos do tipo (5.12). Assim para qualquer $n \geqslant 1$ o modelo (5.12) é muito parecido com o modelo (5.9) para a descrição da cosmologia pôs-inflacionária, incluindo a evolução atual. Com tudo isso, temos que o coeficiente $v$ é o mais relevante para a dinâmica da energia do vácuo para toda a história cósmica após o período inflacionário. No entanto, durante os estágios primitivos do universo o termo $H^{2+n}$ é o fator dominante para a evolução da densidade do vácuo 5.12 dando como resultado uma densidade efetiva $\Lambda(t) \simeq 3 \alpha H^{2+n}(t) / H_{I}^{n}(n \geqslant 1)$, dirigindo a relevância na evolução do sistema aos parâmetros $\alpha$ e $H_{I}$, que podem ser reescritos como um único parâmetro.

\subsection{VÁCUO DINÂMICO E GRUPO DE RENORMALIZAÇÃO}

Na seção anterior sugerimos uma dinâmica da densidade do vácuo como função do parâmetro de Hubble utilizando uma argumentação fenomenológica. No entanto, a dinâmica da energia do vácuo é esperada pela teoria quântica de campos em espaços-tempos curvos [Shapiro and Solà, 2002; Solà, 2008; Shapiro and Solà, 2009; Solà, 2011].

O formalismo do grupo de renormalização na teoria quântica de campos em espaços planos é uma ferramenta muito útil para o estudo de teorias como a QED e a QCD, onde tanto as cargas como as constantes de acoplamento das interações de calibre variam com a escala $\mu$ associada à energia típica do processo. Similarmente, na ação efetiva em teoria quântica de campos em espaço-tempos curvos, $\rho_{\Lambda}$ deve ser uma constante efetiva para baixas energias, mas que na verdade depende da escala de energia $\mu$ do sistema.

No universo em expansão deveríamos esperar que a variação de $\rho_{\Lambda}$ devido a efeitos quânticos dos campos de matéria esteja associada com a mudança na curvatura espaço-temporal, e portanto com a mudança na energia típica do campo gravitacional externo associado com a métrica FRW. Assim esta energia é injetada dentro dos loops dos campos materiais pelo campo gravitacional externo, este deve ser o responsável pela variação no valor esperado da energia do vácuo. Portanto é natural associar tal escala ao parâmetro de Hubble no caso de um universo plano em expansão, já que $3 H^{2}$ é justamente a densidade de energia total do sistema, ver também [Shapiro and Solà, 2002; Shapiro et al., 2005]. 
Dentro do formalismo do grupo de renormalização a taxa de mudança de $\rho_{\Lambda}$ com $\mu=H$ deve satisfazer a equação do grupo de renormalização correspondente:

$$
(4 \pi)^{2} \frac{d \rho_{\Lambda}(\mu)}{d \ln \mu^{2}}=\sum_{m=1,2, \cdots} A_{2 m} \mu^{2 m}=A_{2} \mu^{2}+A_{4} \mu^{4}+A_{6} \mu^{6} \cdots .
$$

O lado direito desta equação define essencialmente a função $\beta$ para a variação de $\rho_{\Lambda}$ devido à renormalização da teoria quântica dos campos materiais. Os coeficientes $A_{2 m}$ recebem contribuições dos loops fermiônicos e bosônicos dos campos com massas $M_{i}$ (menores que a escala da validade da teoria $\left.M_{X}\right)$ que participam do processo [Solà, 2008]. Note-se que apenas as potências pares de $\mu=H$ são permitidas, já que nesta formulação $\rho_{\Lambda}(H)$ é parte da ação efetiva da teoria quântica de campos em espaço-tempos curvos e portanto deve ser uma quantidade covariante [Shapiro and Solà, 2009]. Vale a pena notar que omitimos o termo constante $A_{0}$ em (5.13), já que este deve ser da ordem de $M_{i}^{4}$, o que produziria valores negativos para $\rho_{\Lambda}$ em baixas energias (proporcionais a $\ln \left(\mu^{2} \ll 1\right)<0$ ).

O primeiro termo que contribui na expansão é $A_{2} \mu^{2}$, onde $A_{2}$ possui dimensões de massa ao quadrado, assim $A_{2} \sim \sum_{i} a_{i} M_{i}^{2}$, onde a soma é feita sobre a massa de todos os campos envolvidos no cálculo do valor esperado do vácuo até a escala de energia de validade da teoria (incluindo suas degenerescências). Similarmente, devido a que todos os coeficientes $A_{2 m}$ (exceto $A_{4}$ ) possuem dimensões, é conveniente reescrever eles apropriadamente de uma forma tal que a dimensão de massa fique em evidência. Assim, reescrevemos (5.13) para $\mu=H$ como [Solà, 2008]:

$$
\frac{d \rho_{\Lambda}}{d H^{2}}=\frac{1}{(4 \pi)^{2}} \sum_{i}\left(a_{i} M_{i}^{2}+b_{i} H^{2}+\cdots\right)
$$

Integrando a equação (5.14) podemos obter $\rho_{\Lambda}(H)$, a menos de uma constante aditiva. Em outras palavras, o resultado para $\Lambda(H)=8 \pi G \rho_{\Lambda}(H)$ se adapta bem à forma (5.12) sugerida na seção anterior usando argumentos gerais. No entanto somente potências pares de $H$ são envolvidas no procedimento usando o formalismo do grupo de renormalização, se respeitando a covariância geral da ação efetiva. Fica também evidente que a expansão (5.14) converge rapidamente para baixas energias, onde $H$ é menor, certamente muito menor que a massa de todas as partículas que contribuem para a energia do vácuo. E os termos $H^{2 m}$ além de $H^{2}$ podem contribuir significativamente no lado direito da equação (5.14), durante a historia cósmica, somente na vizinhança da escala de energia $M_{X}$, que é tipicamente de alguns ordens magnitude menor que a escala de Planck, $M_{p l} \sim 10^{19} \mathrm{GeV}$.

No entanto, se queremos ter acesso à física inflacionária, e em geral às eras ainda mais primitivas na evolução do universo, precisamos levar em conta os termos de ordem superior, pelo menos o termo em $H^{4}$, mas como vimos na seção 5.1 qualquer potência $n>2$ vai gerar uma dinâmica da mesma classe que $n=4$. Assim, o termo em $H^{4}$ na expansão da densidade do vácuo deve conter a maior parte da dinâmica a altas energias que vem das contribuições da teoria quântica de campos em espaços-tempos curvos, na descrição semiclássica da gravitação próxima da escala de energia de Planck, mas por baixo dela. Modelos de inflação baseados em termos de ordem superior inspirado no formalismo do grupo de renormalização existem há muito tempo na literatura, ver [Shapiro and Solà, 2002; Solà, 2008] como cenário de unificação da inflação e energia escura. 
Adicionalmente é possível encontrar a relação explícita entre os coeficientes a um loop na equação do grupo de renormalização (5.14) com os coeficientes fenomenológicos introduzidos na seção 5.2, no caso particular em que a potência mais alta na expressão para a energia do vácuo é $H^{4}$. Após integrar a equação do grupo de renormalização (5.14) e comparando com a equação (5.12) obtemos

$$
v=\frac{1}{6 \pi} \sum_{i=f, b} a_{i} \frac{M_{i}^{2}}{M_{p l}^{2}}, \quad \text { e } \quad \alpha=\frac{1}{12 \pi} \frac{H_{I}^{2}}{M_{p l}^{2}} \sum_{i=f, b} b_{i} .
$$

Onde devemos notar que $v$ atua como uma função efetiva $\beta$ para a variação de $\rho_{\Lambda}$ dada pela renormalização para baixas energia, enquanto que $\alpha$ tem o papel equivalente para altas energias. No entanto, os dois coeficientes são preditos para ser naturalmente pequenos, já que $M_{i}^{2} \ll M_{p l}^{2}$ para todas as partículas, inclusive para os campos mais pesados das teorias típicas de grande unificação.

Teoricamente se espera que $v$ seja da ordem de $10^{-2}$ [España-Bonet et al., 2004; Solà and Štefančić, 2005, 2006], permitindo um pequeno comportamento dinâmico na densidade do vácuo para baixas energias, o que é compatível com os ajustes das observações usando dados de supernovas [Shapiro et al., 2003], enquanto que análises incluindo o deslocamento do primeiro pico do espectro de potência da radiação cósmica de fundo e dados de oscilação acústica de bárions, levam a estimativos da ordem de $|v|=\mathcal{O}\left(10^{-3}\right)$ [Basilakos et al., 2009; Grande et al., 2011]. 


\section{UNIVERSO EM EXPANSÃO COM UMA DENSIDADE DO VÁCUO DINÂMICA}

Com base no formalismo do grupo de renormalização que surge naturalmente na teoria de campos de calibre é razoável que a densidade do vácuo (função de Green completa de zero pontos) mude com a escala de energia na que ela é observada, associada no presente trabalho à energia do campo gravitacional externo parametrizado em escalas cosmológicas pelo parâmetro de Hubble, que ao quadrado descreve a densidade de energia total do universo no caso da geometria plana. Assim, escolhendo o parâmetro que define a escala de energia nas equações do grupo de renormalização como sendo o parâmetro de Hubble $H$, mas, desconhecendo a forma exata da equação, expandimos a densidade do vácuo em series de potências de $H$. Adicionalmente, a teoria favorece os coeficientes dos termos com potências pares de $H$, com o que a nossa expressão mais geral para a densidade do vácuo é:

$$
\Lambda=\Lambda(H)=\sum_{m \operatorname{par}} c_{m} H^{m}
$$

A variação na densidade do vácuo exige -via equação de conservação do tensor momento energia- a troca de energia com ao menos uma das componentes materiais presentes no universo (se assumirmos que a parâmetro de acoplamento gravitacional $G$ não apresenta variações nas escalas de energia de interesse). Em particular, com esta forma funcional para a evolução da densidade do vácuo que decresce ao longo da historia cósmica, já que o parâmetro de Hubble decresce sempre para um universo espacialmente plano dominado por fluidos que respeitem a condição de energia fraca $((\rho+\mathcal{P}) \geqslant 0$ e $\rho \geqslant 0)$, obtemos que a componente material que troca energia com o vácuo (relativamente ao caso sem acoplamento) ganha energia durante todo o processo de expansão do universo.

Este modelo, como veremos ao longo do presente capítulo, apresenta todas as vantagens do modelo inflacionário. Mesmo truncando-se a serie de potências no terceiro termo $(m=4)$ ele possui uma saída natural para a era de domínio da radiação não precisando de reaquecimento posterior, e aliviando ainda os problemas da coincidência e da constante cosmológica, já que foi proposto inicialmente para tentar resolver pelo menos este último.

Neste capítulo vamos estudar um universo em expansão composto por um fluido material simples (descrito pela equação de estado $\mathcal{P}(t)=\omega \rho(t)$, com $\omega \geqslant-1 / 3$ ) mais uma densidade do vácuo descrita pela expressão (5.12) (que é o caso particular da expansão (6.1) truncada no terceiro termo):

$$
\Lambda(H)=\Lambda_{c}+3 v H^{2}+3(1-v) \frac{H^{2+n}}{H_{I}^{n}},
$$

onde o coeficiente adimensional $v$ é muito menores do que 1 (5.15) (justificado no capítulo anterior), o prefator $(1-v)$ de $H_{I}^{-n}$ foi introduzido por conveniência matemática, e o parâmetro $H_{I}$ representa 
a função de Hubble característica do período inflacionário. Combinando as equações de Einstein para um universo isotrópico e homogêneo sem curvatura espacial (2.44) com o ansatz (6.2), e usando-se a equação de estado para a densidade do vácuo $\mathcal{P}_{\Lambda}(t)=-\rho_{\Lambda}(t)$, podemos obter a seguinte equação diferencial para a evolução temporal do parâmetro de Hubble:

$$
\frac{d H}{d t}-\xi H^{2}\left\{\frac{\Lambda_{c}}{3(1-v) H^{2}}-1+\left(\frac{H}{H_{I}}\right)^{n}\right\}=0
$$

onde definimos

$$
\xi \equiv \frac{3}{2}(1+\omega)(1-v)
$$

Nota-se que esta equação possui duas soluções particulares constantes: $H_{I} \leftarrow H$ que corresponde ao universo primitivo quando $\Lambda_{c} \ll H^{2}$; por outro lado, para tempos recentes, quando $H \ll H_{I}$ obtemos $H=\left[\Lambda_{c} / 3(1-v)\right]^{1 / 2}$, com o que $\Lambda \approx \Lambda_{c}$ comporta-se como uma constante cosmológica efetiva. Também, usando-se a equação (6.3) o parâmetro de desaceleração $q \equiv-a \partial_{t}^{2} a /\left(\partial_{t} a\right)^{2}=-1-\partial_{t} H / H^{2}$ é dado por

$$
q(H)=-\xi\left\{\frac{\Lambda_{c}}{3(1-v) H^{2}}-1+\left(\frac{H}{H_{I}}\right)^{n}\right\}-1 .
$$

Apresentamos a seguir as diferentes eras da cosmologia geradas pela presença de um vácuo dinâmico da forma (6.2), começando pela instável fase inflacionária dirigida pelo grande valor de $H_{I}$, da ordem da escala da grande unificação; seguida pelas eras de domínio dos fluidos materiais após a densidade do vácuo cair rapidamente no fim da inflação aquecendo o universo; e finalmente um universo recente acelerado, e dominado pela densidade do vácuo remanescente (efetivamente constante, mas com uma pequena dinâmica devida ao parâmetro $v$ ).

\subsection{UNIVERSO PRIMORDIAL}

Durante os estágios primordiais do universo o parâmetro $\Lambda_{c}$ é desprezível (se comparado com os termos em $H^{n}$ na expressão para a densidade do vácuo). Com isso, a equação (6.3) para a evolução do parâmetro de Hubble torna-se

$$
\frac{d H}{d t}+\xi H^{2}\left\{1-\left(\frac{H}{H_{I}}\right)^{n}\right\}=0 .
$$

A integração da equação acima produz a seguinte dependência do parâmetro de Hubble com o fator de escala:

$$
H=\frac{H_{I}}{(1+\chi)^{1 / n}}, \quad \text { com } \quad \chi \equiv \chi(a)=D a^{n \xi},
$$

onde $H_{I}$ é o valor do parâmetro de Hubble associado ao estágio inflacionário, $D$ é uma constante de integração. Nota-se que se $D=0$ a solução permanece sempre De Sitter, enquanto que para $D \neq 0$ o universo começa com uma era aproximadamente de Sitter e evolui continuamente para a era de domínio do fluido material. 
Usando as equações de Einstein e a solução acima podemos obter as seguintes expressões para as densidades do vácuo, do fluido material e para a densidade de energia total:

$$
\rho_{\Lambda}(a)=\rho_{I} \frac{1+v \chi}{(1+\chi)^{1+2 / n}}, \quad \rho(a)=\rho_{I} \frac{(1-v) \chi}{(1+\chi)^{1+2 / n}}, \quad \rho_{\mathrm{T}}(a)=\rho_{I} \frac{1}{(1+\chi)^{2 / n}} .
$$

onde definimos $\rho_{I} \equiv 3 H_{I}^{2} / 8 \pi G$ como sendo a densidade critica durante o período inflacionário. Dito período é caracterizado por uma expansão acelerada (4.9) ou, equivalentemente, pelo decrescimento no raio comóvel de Hubble (4.8) (que é o horizonte aparente para um universo plano em coordenadas comoveis). Com isso podemos definir o fim do período inflacionário pela condição:

$$
-\frac{6}{a} \frac{d^{2} a}{d t^{2}}=\left.8 \pi G\left(\rho_{T}+3 \mathcal{P}_{T}\right)\right|_{a=a_{\star}}=-\left.6 H^{2}\left(1+a \frac{H^{\prime}}{H}\right)\right|_{a=a_{\star}} \equiv 0,
$$

onde definimos $a=a_{\star}$ como sendo o fator de escala no fim da inflação. Usando a condição acima podemos expressar a constante de integração $D$ em função do fator de escala no fim da inflação como sendo:

$$
D=\frac{a_{\star}^{-n \xi}}{\xi-1}, \quad \text { assim } \quad \chi(a)=\frac{1}{\xi-1}\left(\frac{a}{a_{\star}}\right)^{n \xi}
$$

No caso em que $\omega=1 / 3$ a condição (6.9), que define o fim do período inflacionário, é equivalente à condição de igualdade entre a densidade do vácuo e a densidade de energia da radiação $\rho\left(a_{\star}\right)=\rho_{\Lambda}\left(a_{\star}\right)$.

Derivando a expressão para a densidade de energia do fluido material podemos ver que este possui um máximo (ver figura 6.1) para:

$$
a_{\rho \max }=a_{\star}\left(\frac{n(\xi-1)}{2}\right)^{1 /(n \xi)} \stackrel{\omega=1 / 3}{\longrightarrow} a_{\star}\left(\frac{n(1-2 v)}{2}\right)^{1 /(2 n(1-v))} .
$$

Usando a variável auxiliar $u=-\chi^{-1}$ podemos transformar a equação (6.7) em:

$$
\frac{d t}{d u}=-\frac{1}{n \xi H_{I}} u^{-(1+1 / n)}(u-1)^{1 / n}
$$

cuja segunda derivada pode ser reescrita como:

$$
u(1-u) \frac{d^{2} t}{d u^{2}}+\left(1+\frac{1}{n}-u\right) \frac{d t}{d u}=0 .
$$

Esta é a equação hipergeométrica com parâmetros $\alpha_{1}=0, \alpha_{2}=0$, e $\alpha_{3}=1+1 / n$; e sua integração produz:

$$
t(u)=B-C u^{-1 / n} F\left[-\frac{1}{n},-\frac{1}{n}, 1-\frac{1}{n}, u\right],
$$

onde $B$ e $C$ são constantes de integração. Usando as relações de Euler, a solução acima pode ser reescrita como:

$$
t(a)=B+\frac{(1+\chi)^{\frac{1+n}{n}}}{\xi H_{I} \chi} F\left[1,1,1-\frac{1}{n}, \frac{-1}{\chi}\right]
$$

onde $C$ foi escolhido para reobter a expressão (6.7) para o parâmetro de Hubble, enquanto a escolha da constante $B$ fixa a origem do tempo. Por exemplo, a escolha de $B=F\left[1,1, \frac{n-1}{n}, 1-\xi\right] \times H_{I}^{-1} \xi(1-$ 

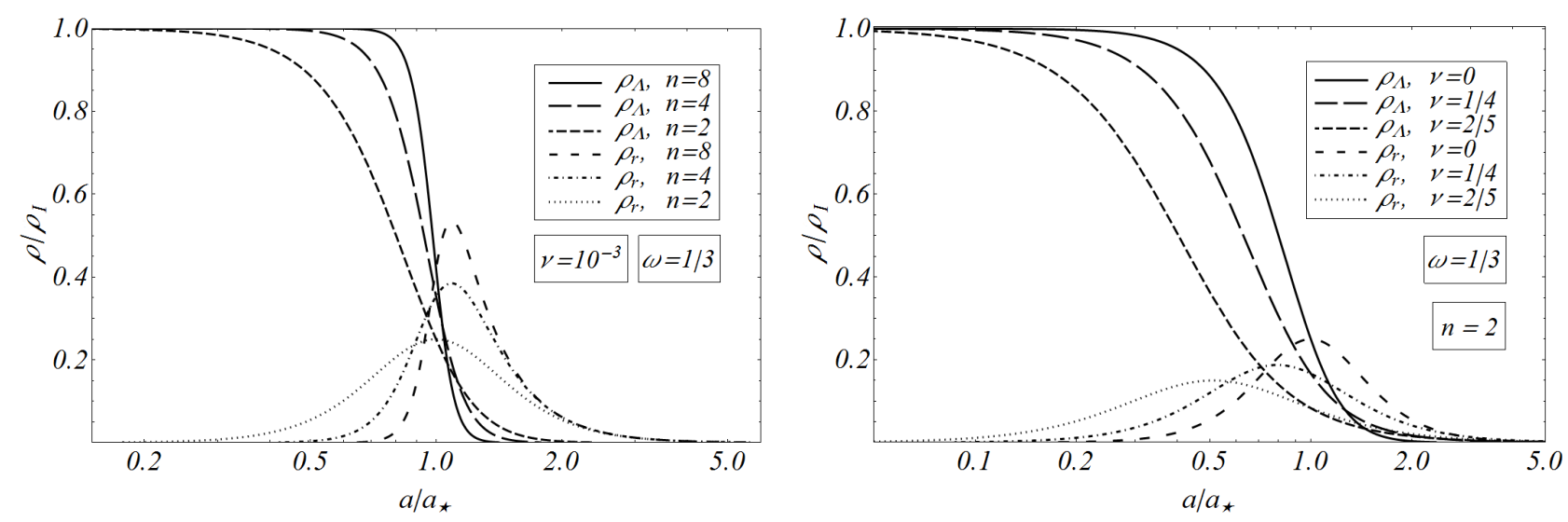

Figura 6.1: Evolução das densidades no universo primordial com $\Lambda=3 v H^{2}+3(1-v) H^{2+n} / H_{I}^{n}$. Mostra-sem as densidades de energia da radiação e do vácuo para diferentes valores dos parâmetros $n$ e $v$, estas estão normalizadas em relação à densidade crítica durante o período primordial acelerado $\rho_{I}$. Na figura se mostra que o crescimento na densidade de energia da radiação durante o período inflacionário, também como o decrescimento na densidade do vácuo e da radiação após a inflação são acentuados com o aumento do parâmetro $n$ ou com a diminuição do parâmetro $v$.

$\xi)^{-1}$ é equivalente a tomar a origem do tempo no fim do período inflacionário $\left(t\left(a_{\star}\right) \equiv 0\right)$, e para $n=2$ a solução acima assume a forma:

$$
t(a)=B+\frac{1}{\xi H_{I}} \sqrt{1+\chi}-\frac{1}{\xi H_{I}} \operatorname{ArcCoth}[\sqrt{1+\chi}] .
$$

\subsubsection{Inflação e período de domínio da radiação}

Durante a era De Sitter e a era de domínio da radiação, onde $\Lambda_{c} \ll H^{2}$, a solução (6.7) à equação (6.3) para $\omega=1 / 3$ e $\Lambda_{c}=0$ torna-se:

$$
H(a)=\frac{H_{I}}{(1+\chi)^{1 / n}}
$$

onde $\chi(a)=\left(a / a_{\star}\right)^{2 n(1-v)} /(1-2 v)$. Integrando esta equação em relação ao fator de escala obtém-se a expressão para o tempo cósmico em função do fator de escala (6.15) com $\xi=2(1-v)$. Usando as equações de Einstein podemos obter as expressões (6.8) para a densidade do vácuo, a densidade de energia da radiação e a densidade total; onde podemos ver que $\rho_{r} / \rho_{\Lambda} \propto a^{2 n(1-v)} \rightarrow 0$ quando $a \rightarrow 0$, assim o universo primitivo é dominado pelo vácuo, gerando uma expansão acelerada.

Para $a \ll a_{\star}$ a solução (6.17) pode ser aproximada pelo valor constante $H \approx H_{I}$, a densidade do vácuo também permanece quase constante $\rho_{\Lambda} \approx \rho_{I}$, enquanto a densidade de energia da radiação permanece desprezível, mas se incrementando como $\rho_{r} \simeq \rho_{\Lambda}\left(a / a_{\star}\right)^{2 n(1-v)}(1-v) /(1-2 v)$. Este período corresponde ao estágio primitivo de Sitter, onde

$$
a(t) \propto e^{H_{I} t}
$$




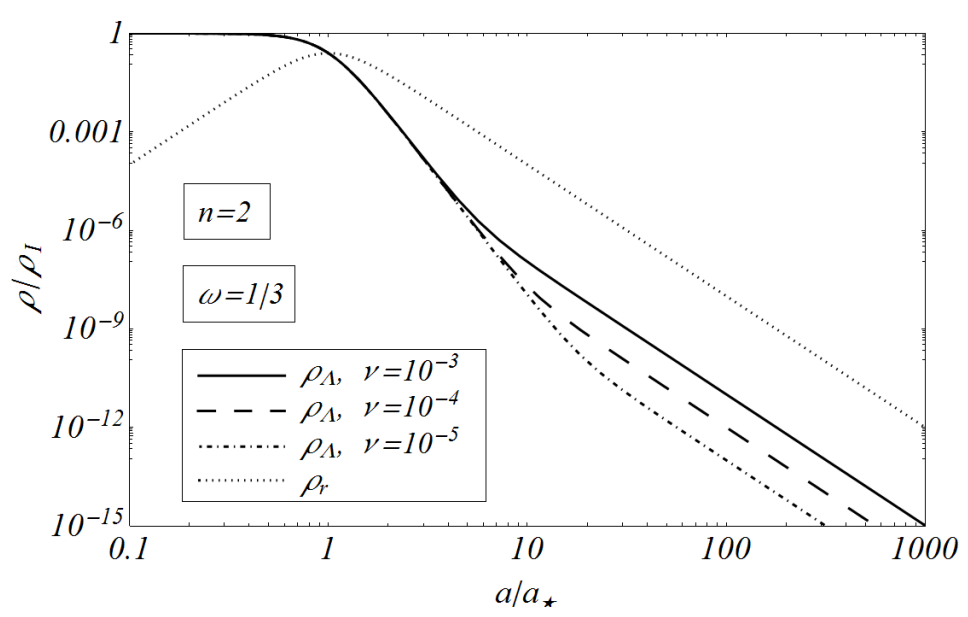

Figura 6.2: Evolução das densidades no universo primordial com $\Lambda=3 v H^{2}+3(1-v) H^{2+n} / H_{I}^{n}$. Nesta figura podemos ver que a densidade do vácuo decresce mais rapidamente que a densidade de energia da radiação logo após o período inflacionário, permitindo o estabelecimento do domínio da radiação. Depois desta fase, a densidade do vácuo passa a imitar a diluição da densidade de energia da radiação a menos um fator $v$. Adicionalmente valores de $v \ll 1$ afetam muito pouco a evolução da densidade de energia da radiação.

enquanto o universo sofre o processo de inflação. O resultado (6.18) pode ser obtido expandindo a solução (6.15) ao redor de $a \ll a_{\star}$ :

$$
H_{I} t \approx \frac{1}{2 n(1-v)}\left(C_{n}+\ln \left[\frac{\left(a / a_{\star}\right)^{2 n(1-v)}}{1-2 v}\right]\right),
$$

onde $C_{n}$ é uma constante que depende de $n$.

A conclusão das considerações acima é que para $D \neq 0$ o universo começa com um estágio acelerado, e não apresenta uma singularidade inicial, evitando o problema do horizonte. Um modo diferente de ver isto é que um raio de luz emitido em $a \rightarrow 0$ terá viajado até o fim da inflação $a_{\star}$ uma distância física $\left(a_{\star} / H_{I}\right) \int_{a \rightarrow 0}^{a_{\star}} d a / a^{2}$ diverge, implicando a ausência de horizontes de partículas para observadores comoveis. Portanto as interações locais conseguem homogeneizar o universo.

Para $a \gg a_{\star}$ a solução (6.17) pode ser aproximada como

$$
H \approx H_{I}(1-2 v)^{1 / n}\left(\frac{a}{a_{\star}}\right)^{-2(1-v)} .
$$

Similarmente, da equação (6.15) podemos obter

$$
t \propto a^{2(1-v)}
$$

enquanto a densidade de energia da radiação decai como $\rho_{r} \propto(1-v) a^{-4(1-v)}$, o que restringe o valor do parâmetro $v$ como sendo muito menor que 1 -se queremos que a era dominada pela radiação não seja muito diferente da apresentada pelo modelo $\Lambda$ CDM-. Também podemos ver em (6.8) que a densidade do vácuo apresenta um decrescimento similar $\rho_{\Lambda} \propto v a^{-4(1-v)}$, mas sua densidade é suprimida pelo fator $\rho_{\Lambda} / \rho_{r} \approx v /(1-v)(\operatorname{com}|v| \ll 1)$ se comparada com a densidade de energia da radiação. Este é o caso 
oposto do universo primordial, quando $a \ll a_{\star}$, onde a densidade do vácuo é da ordem de $\rho_{I}$ enquanto a densidade de energia da radiação é fortemente suprimida pelo fator $a^{2 n(1-v)}$.

Durante o final da era inflacionária $\left(a<a_{\star}\right)$ podemos ver que se tem uma forte aumento na densidade de energia da matéria relativística $\rho_{r} \propto a^{2 n(1-v)}(\dot{\rho}>0)$, enquanto se tem o decrescimento usual $\rho_{r} \propto a^{-4(1-v)}$ (equivalente ao modelo $\Lambda \mathrm{CDM}$ para $|v| \ll 1$ ) quando o universo é dominado pela radiação $\left(a \gg a_{\star}\right)$.

\section{Solução ao problema de reaquecimento}

No caso em que $\omega=1 / 3$, obtemos um aumento contínuo na densidade da matéria relativística $\rho_{r} \propto$ $a^{2 n(1-v)}$ durante a era deflacionaria, e diluição $\rho_{r} \propto a^{-4(1-v)}$ durante a subsequente era da radiação. Assumindo uma expansão adiabática durante as duas eras, a densidade de energia da radiação escala como $\rho_{r} \sim \mathrm{T}_{r}^{4}$ com sua própria temperatura [Lima, 1996, 1997]. Assim, obtemos que a temperatura da radiação cresce como $\mathrm{T}_{r} \propto a^{n(1-v) / 2}$ durante o período inicial De Sitter (expansão acelerada) se a entropia especifica por partícula permanece constante durante dito período. Com isto, a radiação no universo é naturalmente aquecida durante todo o período primordial acelerado, principalmente no fim deste período. Em particular, temos que a densidade de energia da radiação no fim da inflação (quando $\left.a=a_{\star}\right)$ é da ordem de

$$
\rho_{r}\left(a_{\star}\right)=\frac{\rho_{I}}{2^{1+2 / n}}\left(\frac{1-2 v}{1-v}\right)^{2 / n} \lesssim \frac{\rho_{I}}{4} \quad \text { para } \quad n \gtrsim 2
$$

Isto é equivalente a uma temperatura dos fótons da ordem de $\mathrm{T}_{r}^{\star} \lesssim 10^{20} \mathrm{~K}$, se a escala de energia da inflação é assumida como sendo a escala de energia da grande unificação, da ordem de $10^{16} \mathrm{GeV}$. Assim o modelo que estamos considerando não precisa de um reaquecimento posterior do universo, dando origem a uma evolução pós-inflacionária equivalente ao modelo $\Lambda \mathrm{CDM}$, para valores de $v \ll 1$.

Depois da era de Sitter, a temperatura decresce continuamente durante o processo de expansão como $\mathrm{T}_{r} \approx \mathrm{T}_{r}^{\star}\left(a / a_{\star}\right)^{-(1-v)}=\mathrm{T}_{r}^{0}\left(a / a_{0}\right)^{-1(1-v)}$, o que não se afasta muito de comportamento $1 / a$ para $|v| \ll 1$, como deve acontecer em ausência de interações durante uma expansão adiabática. De acordo com isto, a densidade numero comóvel de fótons escala como $n_{\gamma} \sim T^{3} \propto a^{-3(1-v)}$, o que mostra um pequeno desvio do modelo $\Lambda$ CDM.

\section{Parâmetro de desaceleração para o universo primordial}

No caso em que $\Lambda_{c} \ll H^{2}$ e $\omega=1 / 3$ (solução (6.7)), o parâmetro de desaceleração (6.5) assume a forma:

$$
q(a)=\xi \frac{\chi}{1+\chi}-1=\frac{(1-2 v) \chi-1}{1+\chi}, \quad \text { com } \quad \chi=\frac{1}{1-2 v}\left(\frac{a}{a_{\star}}\right)^{2 n(1-v)},
$$

variando de $q_{I} \approx-1$ quando $a \rightarrow 0$, até o valor positivo $(q=1-2 v$ próximo ao valor usual para $v \ll 1$ ) durante o período dominado pela radiação quando $a \gg a_{\star}$. Esta transição primordial (pt) entre o período de expansão acelerada e o período desacelerado dominado pela radiação (quando ainda $H \gg H_{0}$ ) ocorre quando o fator de escala assume o valor $a_{\star}$. 

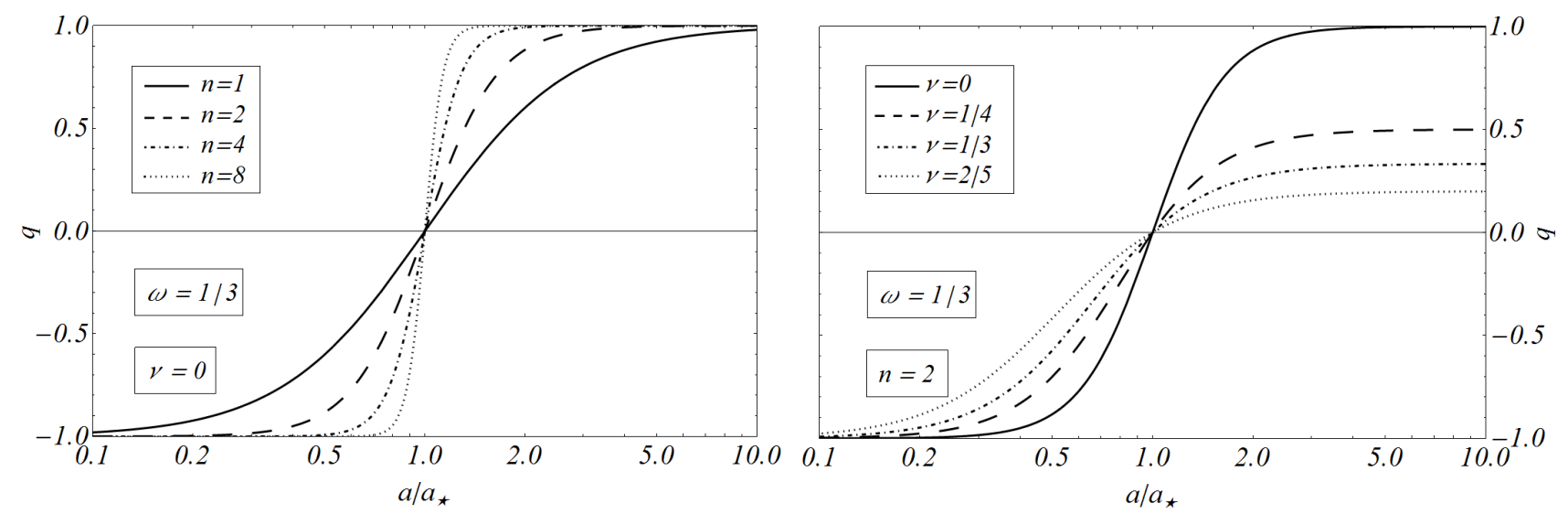

Figura 6.3: Parâmetro de desaceleração durante as eras primordiais com $\Lambda=3 v H^{2}+3(1-v) H^{2+n} / H_{I}^{n}$. Podemos ver que a transição entre o período acelerado $q \sim-1$ e o período desacelerado $q \sim(1-2 v)$ ocorre mais rapidamente para valores maiores de $n$. Sabendo que durante o período de domínio da radiação no modelo $\Lambda \mathrm{CDM}$ o parâmetro de desaceleração é $q_{r}=1$ temos que para que o presente modelo não seja muito diferente do modelo $\Lambda \mathrm{CDM}$, durante o dito período, oo parâmetro $v$ deve ficar restrito a valores $|v| \ll 1$, ou seja $\xi \approx 2$.

\subsection{POTENCIAL EFETIVO EQUIVALENTE À DENSIDADE TOTAL NO UNIVERSO PRIMORDIAL}

Na seção 5.3 motivamos o presente modelo usando a estrutura geral da ação efetiva em teoria quântica de campos em espaços-tempos curvos, e sugerindo que a equação do grupo de renormalização (5.14) pode conduzir naturalmente à expressão geral para a densidade do vácuo (5.12), e assumiremos que qualquer tipo de evolução para a densidade do vácuo pode ser descrita nesta forma [Solà and Štefančić, 2005, 2006]. Esto pode ser útil para considerações fenomenológicas na descrição da energia escura. Na forma usual, as expressões para a densidade de energia e a pressão de um campo escalar com potencial $V$ são $\rho_{\mathrm{T}} \rightarrow \rho_{\varphi}=\left(\partial_{t} \varphi\right)^{2} / 2+V(\varphi)$ e $\mathcal{P}_{\mathrm{T}} \rightarrow \mathcal{P}_{\varphi}=\left(\partial_{t} \varphi\right)^{2} / 2-V(\varphi)$, e substituídas dentro das equações de Friedmann (2.44) produzem $4 \pi G\left(\partial_{t} \varphi\right)^{2}=-\partial_{t} H$ e

$$
V_{\mathrm{eff}}(a)=\frac{3 H^{2}}{8 \pi G}\left(1+\frac{\partial_{t} H}{3 H^{2}}\right)=\frac{3 H^{2}}{8 \pi G}\left(1+\frac{1}{3} \frac{d \ln H}{d \ln a}\right) .
$$

O potencial efetivo pode ser obtido partindo da expressão para o parâmetro de Hubble durante o universo primordial (6.7). Fazendo a conta desconsiderando as correções da ordem de $\mathcal{O}(v)$ obtemos

$$
V_{\mathrm{eff}}(a)=\rho_{I} \frac{1+\chi \xi / 3}{[1+\chi]^{1+2 / n}} \quad \stackrel{\omega \rightarrow 1 / 3}{\underset{v \rightarrow 0}{\longrightarrow}} \quad \rho_{I} \frac{1+\left(a / a_{\star}\right)^{2 n} / 3}{\left[1+\left(a / a_{\star}\right)^{2 n}\right]^{1+2 / n}}
$$

O caso em que $n=2$, que corresponde a ter o termo $H^{4}$ como maior potencia de $H$ na expressão para a densidade do vácuo (6.2) produz

$$
\left.V_{\text {eff }}(a)\right|_{n=2}=\rho_{I} \frac{1+\left(a / a_{\star}\right)^{4} / 3}{\left[1+\left(a / a_{\star}\right)^{4}\right]^{2}} .
$$

Esta forma específica, obtida previamente por [Lima et al., 2013], é um caso particular do potencial efetivo geral (6.25). A partir da expressão geral é claro que o potencial permanece constante, $V_{\text {eff }} \sim \rho_{I} / \alpha$, enquanto $a \ll a_{\star}$ (ou seja, antes da transição do fim da inflação). No entanto, depois de que a transição 
para a era de domínio da radiação ocorre (quando $a \gg a_{\star}$ ) o potencial efetivo (6.25) decresce na forma $V(a) \sim a^{-4}$, valido para todo $n$. Apresentando uma saída natural do período acelerado para a fase dominada pela radiação para qualquer valor de $n$.
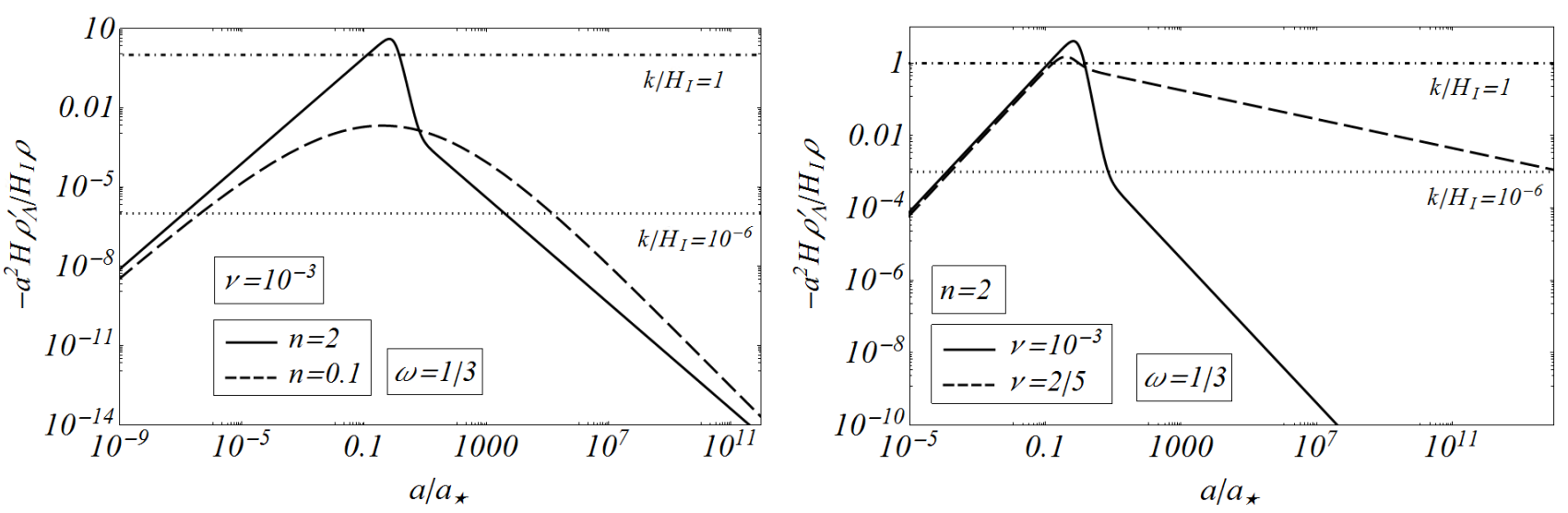

Figura 6.4: Termos que dominam a evolução das perturbações escalares durante o período deflacionário. Mostra-se como o termo diretamente relacionado à variação na densidade do vácuo adquire relevância durante o período de transição entre o estagio inflacionário e o estágio dominado por matéria relativística.
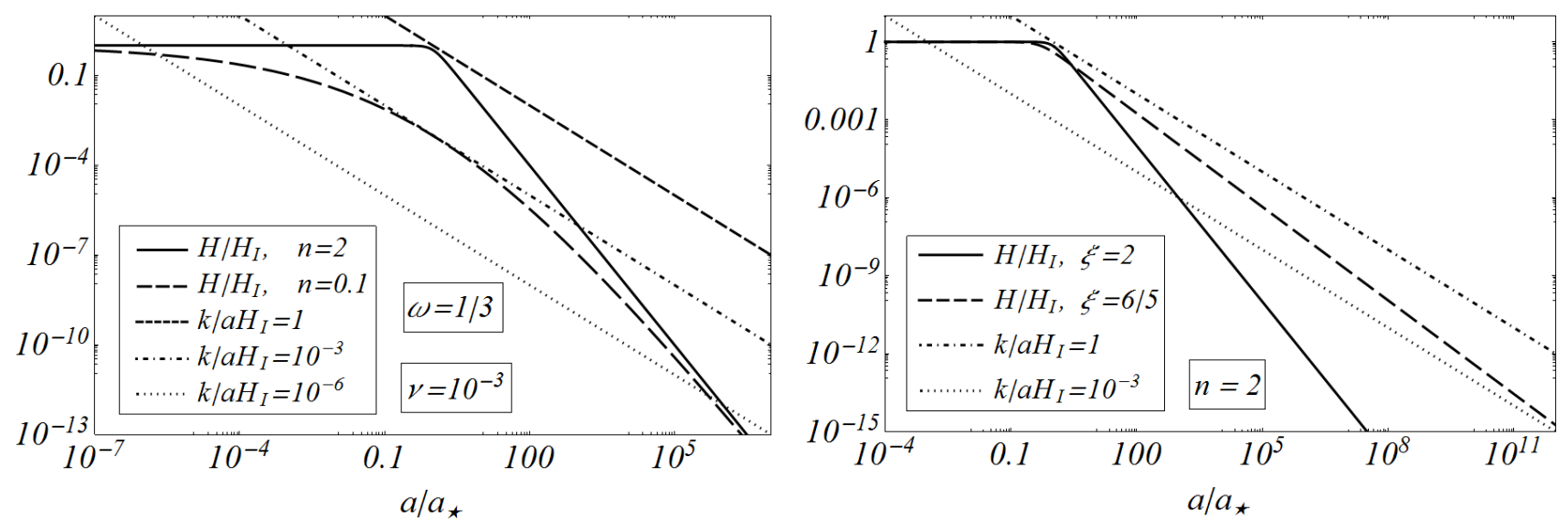

Figura 6.5: Como as escalas saem e entram no horizonte por causa da inflação.

Mostra-se como as diferentes escalas que estão dentro do horizonte no começo da inflação saem durante o dito período (se são grandes o suficiente), devido ao decrescimento do raio de Hubble comóvel durante o período de expansão acelerada, e entram novamente no horizonte devido ao subsequente período de expansão desacelerada, quando o universo é dominado pelos fluidos materiais.

\subsection{PERTURBAÇÕES ESCALARES NO UNIVERSO PRIMORDIAL}

As equações para a evolução das perturbações escalares (componentes da equação de conservação do tensor momento energia (3.43) e a componente espaço-temporal das equações de Einstein (3.34d)) para um universo formado por um vácuo dinâmico e radiação, desconsiderando perturbações anisotrópicas no sistema $(\sigma=0)$, se escrevem em função do fator de escala como:

$$
a^{2} H \delta^{\prime}=-\frac{4}{3} \theta+4 a^{2} H \phi^{\prime}+a^{2} H \frac{\rho_{\Lambda}^{\prime}}{\rho} \delta
$$




$$
\begin{gathered}
a^{2} H \theta^{\prime}=k^{2} \frac{\delta}{4}+k^{2} \phi+a^{2} H \frac{\rho_{\Lambda}^{\prime}}{\rho} \theta, \\
16 \pi G a \frac{\rho}{3} \theta=H k^{2}\left(a \phi^{\prime}+\phi\right) .
\end{gathered}
$$

Na figura 6.4 comparam-se os coeficientes mais relevantes destas equações durante os períodos de inflação e de domínio da radiação. Podemos ver, nesta figura, que o termo relacionado com variação na densidade do vácuo é mais relevante durante o período de transição entre os dois períodos. Depois de analisar a relevância dos diferentes termos nas equações (6.27) podemos estabelecer as condições iniciais, para resolver tais equações, no caso dos modos $k$ que estão bem dentro do horizonte no começo do período inflacionário. Depois de aplicar as devidas simplificações para os modos que inicialmente $\left(a \ll a_{\star}\right)$ estão dentro do horizonte $\mathcal{H} \ll k$, as equações (6.27) podem ser reescritas como:

$$
a \phi^{\prime}+\phi=0, \quad \frac{\delta}{4}+\left.\phi\right|_{\tilde{a}}=0, \quad \theta-\left.6(1+n(1-v)) H_{I} a \phi\right|_{\tilde{a}}=0,
$$

onde índice denota a derivada em relação ao fator de escala, e ã é o fator de escala no começo do período inflacionário $\left(\tilde{a} \leqslant a_{\star} 10^{-28}\right.$ para resolver o problema do horizonte, ver equação (4.12)). Estas expressões definem as condições iniciais para as perturbações $\delta$ e $\theta$ em função da amplitude inicial da perturbação $\phi$, enquanto que a solução à equação que define a evolução da perturbação $\phi$ para os modos de sub-horizonte no começo da inflação é:

$$
\phi\left(a \ll a_{\star}\right)=\frac{c_{9}}{a} .
$$

Integrando numericamente as equações (6.27) com as condições iniciais (6.28 - 6.29) podemos obter a evolução para as perturbações escalares mostrada na figura 6.7 .

\subsubsection{Espectro de potências primordial}

No painel direito da figura 6.5 podemos ver que para um valor fixo do parâmetro $n$ o instante em que uma escala arbitrária sai do horizonte não é sensivelmente modificado pela variação do parâmetro $\xi$, mas a entrada no horizonte após o período inflacionário é fortemente retrasada para valores menores do parâmetro $\xi$, mas a exigência de uma evolução no background pós inflacionária similar ao modelo $\Lambda \mathrm{CDM}$ restringe o parâmetro $\xi \sim 2$ para $\omega=1 / 3$.

Já no caso de um parâmetro $\xi$ fixo (painel esquerdo da figura 6.5), a redução no valor do parâmetro $n$ atrasa (adianta) a saída (entrada) das escalas no horizonte durante (após) o período inflacionário, fazendo com que as perturbações escalares com escalas cada vez menores possuam uma amplitude menor na hora de entrar no horizonte, o que faz com que o espectro de potências inicial possua um índice espectral $n_{s}<1$. Enquanto que para valores cada vez maiores do parâmetro $n$ o índice espectral se aproxima arbitrariamente do valor $n_{s}=1$.

Antes que a escala pivô $k_{p}$ atravesse o horizonte de Hubble, para $a<a_{\star} \times 10^{-31}$, podemos aproximar a evolução da perturbação métrica $\Phi$ dada pela expressão (6.29). Avaliando esta expressão no instante 


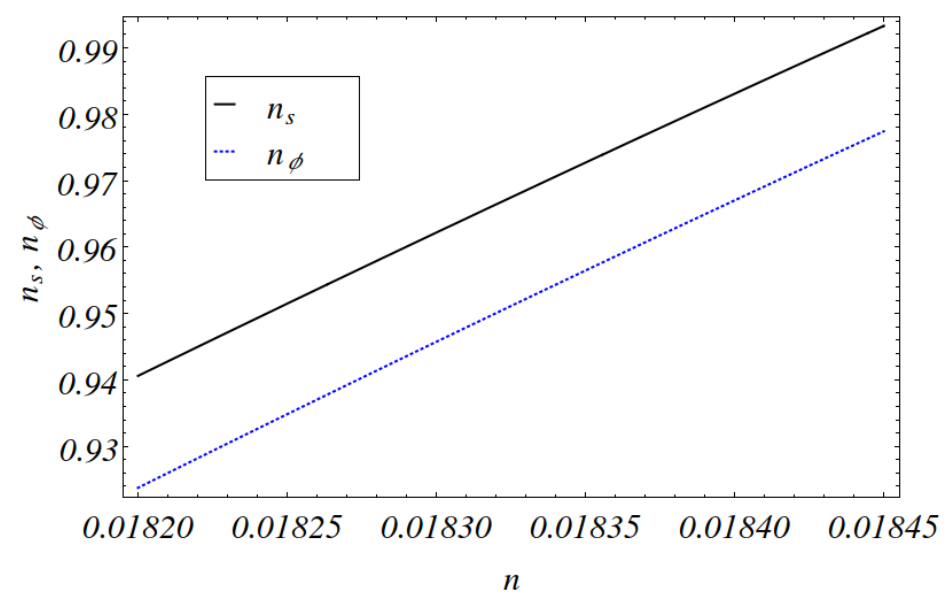

Figura 6.6: Índice espectral das perturbações escalares no modelo deflacionário.

Mostra-se o índice espectral das perturbação métrica $n_{\phi}$ no instante em que a escala pivô $k_{p}$ sai do horizonte durante o estagio primordial acelerado, como também o índice espectral da perturbação na densidade de energia da matéria $n_{s}$ no instante em que a escala pivô re-entra no horizonte, como uma função do parâmetro $n$, para o modelo deflacionário $\operatorname{com} v=0$.

em que cada escala sai do horizonte $\mathcal{H}=k$ podemos obter o espectro de potências primordial das perturbações métricas mostrado na figura (6.6) no casso em que $v=0$.

No caso em que $v=0$ e $\omega=1 / 3$ podemos escrever (6.7):

$$
H(a)=\frac{H_{I}}{\left[1+\left(a / a_{\star}\right)^{2 n}\right]^{1 / n}}
$$

e no instante em que $\mathcal{H}=k$ obtemos:

$$
a(\mathcal{H}=k)=a_{\star}\left(\left(\frac{\mathcal{H}_{\star}}{k}\right)^{n}-\sqrt{\left(\frac{\mathcal{H}_{\star}}{k}\right)^{2 n}-1}\right)^{1 / n}
$$

desta forma, no instante em que as perturbações cruzam o horizonte $\left.\phi\right|_{\mathcal{H}}=c_{9} / a(k=\mathcal{H})$, e seu espectro sobre as mesmas condições é:

$$
P_{\phi}(k) \delta^{3}\left(k-k^{\prime}\right)(2 \pi)^{-3}=\left\langle\phi^{*}(k) \phi\left(k^{\prime}\right)\right\rangle, \quad \Delta_{\phi}=\frac{k^{3}}{2 \pi^{2}} P(k)=\left.\frac{\text { cte } k^{3}}{a^{2}}\right|_{k=\mathcal{H}},
$$

com o que podemos calcular o índice espectral na escala pivô como sendo:

$$
n_{\phi} \equiv \frac{\partial \ln \Delta_{\phi}}{\partial \ln k}+1=\frac{4 \sqrt{x^{-2 n}-1}-2 x^{-n}}{\sqrt{x^{-2 n}-1}}
$$


onde $x \equiv k / \mathcal{H}_{\star}$, e sua variação ao redor da escala pivô:

$$
\begin{aligned}
\frac{\partial n_{\phi}}{\partial \ln k} & =\frac{-2 n x^{n}}{\sqrt{x^{-2 n}-1}\left(x^{2 n}-1\right)}, \\
\frac{\partial^{2} n_{\phi}}{\partial \ln k^{2}} & =-\frac{2 n^{2} x^{n}\left(1+2 x^{2 n}\right)}{\sqrt{x^{-2 n}-1}\left(x^{2 n}-1\right)^{2}} .
\end{aligned}
$$

Na figura 6.6 se mostra o índice espectral $n_{\phi}$ das perturbações do campo $\phi$ no instante em que a escala pivô cruza o horizonte, em função do parâmetro $n$.

Agora podemos calcular o invariante $\zeta$ no instante em que cada escala cruza o horizonte

$$
\left.\zeta \equiv \frac{3 \phi+a \phi^{\prime}}{2}\right|_{k=\mathcal{H}}=\left.\frac{\phi}{2}\left(3-\frac{\partial \ln \phi}{\partial \ln k}\right)\right|_{k=\mathcal{H}},
$$

se agora usamos a definição do índice espectral $n_{\phi}$ obtemos:

$$
n_{\phi}-1 \equiv \frac{\partial \ln \left(k^{3} \phi^{2}\right)}{\partial \ln k}=3+2 \frac{\partial \ln \phi}{\partial \ln k}, \quad \rightarrow \quad \zeta=\left.\phi\left(\frac{5}{2}-\frac{n_{\phi}}{4}\right)\right|_{k=\mathcal{H}}{ }^{\prime}
$$

e graças a que $\dot{\zeta}=0$ fora do horizonte ${ }^{1}$, podemos recalcular-lho no instante em que as escalas entram novamente no horizonte durante o período de domínio da radiação:

$$
\zeta=\phi^{(r)}+\left.\left.\frac{\mathcal{H}}{k^{2}} \theta_{\gamma}^{(r)}\right|_{k=\mathcal{H}} \approx \frac{3}{2} \phi^{(r)}\right|_{k=\mathcal{H}},
$$

onde usamos as condições iniciais para as perturbações fora do horizonte no calibre longitudinal (definido como $B=0$ e $\mu=0$ em (3.7)) descritas por [Ma and Bertschinger, 1995]. O super-índice ( $r$ ) denota o período de domínio da radiação. Se agora usamos a equação de Poisson (componente 00 da equação de Einstein perturbada no calibre longitudinal para pequenas escalas $k \gg \mathcal{H}$ ) podemos relacionar a perturbação $\delta_{m}$ com o invariante $\zeta$ no instante em que cada escala cruza o horizonte durante o período de domínio da radiação:

$$
\zeta=\left.\frac{3}{2} \phi^{(r)}\right|_{k=\mathcal{H}}=-\left.\frac{2 \pi G a^{2} \rho_{\gamma}^{(r)}}{k^{2}} \delta_{m}^{(r)}\right|_{k=\mathcal{H}} \approx-\left.\frac{3}{4} \delta_{m}^{(r)}\right|_{k=\mathcal{H}} .
$$

Igualando as expressões (6.36) e (6.38) podemos relacionar o espectro inicial (no instante em que as escalas entram no horizonte durante o período de domínio da radiação) da perturbação $\delta_{m}^{(r)} \operatorname{com~o}$ espectro primordial (no instante em que as escalas saem do horizonte durante o período inflacionário) da perturbação $\phi$ :

$$
\left.\left(10-n_{\phi}\right) \phi^{(i n f)}\right|_{k=\mathcal{H}} \approx-\left.3 \delta_{m}^{(r)}\right|_{k=\mathcal{H}}, \quad \rightarrow \quad 9 \Delta_{m}=\left(10-n_{\phi}\right)^{2} \Delta_{\phi},
$$

\footnotetext{
$1 \overline{\dot{\zeta}}=0$ é uma aproximação, que perde validade durante um curto período no fim da inflação, quando uma grande quantidade de radiação é produzida (ver figura 6.2 , onde $d \rho / d a \lesssim \rho_{T}$ ), e portanto a equação (3.42) se torna $\dot{\zeta} \mathcal{H}=4 \pi G a^{2} \tau \delta S$.
} 
e com isto obtemos que

$$
\begin{gathered}
n_{s} \equiv 1+\frac{\partial \ln \Delta_{m}}{\partial \ln k}=n_{\phi}-\frac{2}{\left(10-n_{\phi}\right)} \frac{\partial n_{\phi}}{\partial \ln k} \\
\frac{\partial n_{s}}{\partial \ln k}=\frac{\partial n_{\phi}}{\partial \ln k}-\frac{2}{\left(10-n_{\phi}\right)} \frac{\partial^{2} n_{\phi}}{\partial \ln k^{2}}-\frac{2}{\left(10-n_{\phi}\right)^{2}}\left(\frac{\partial n_{\phi}}{\partial \ln k}\right)^{2}
\end{gathered}
$$
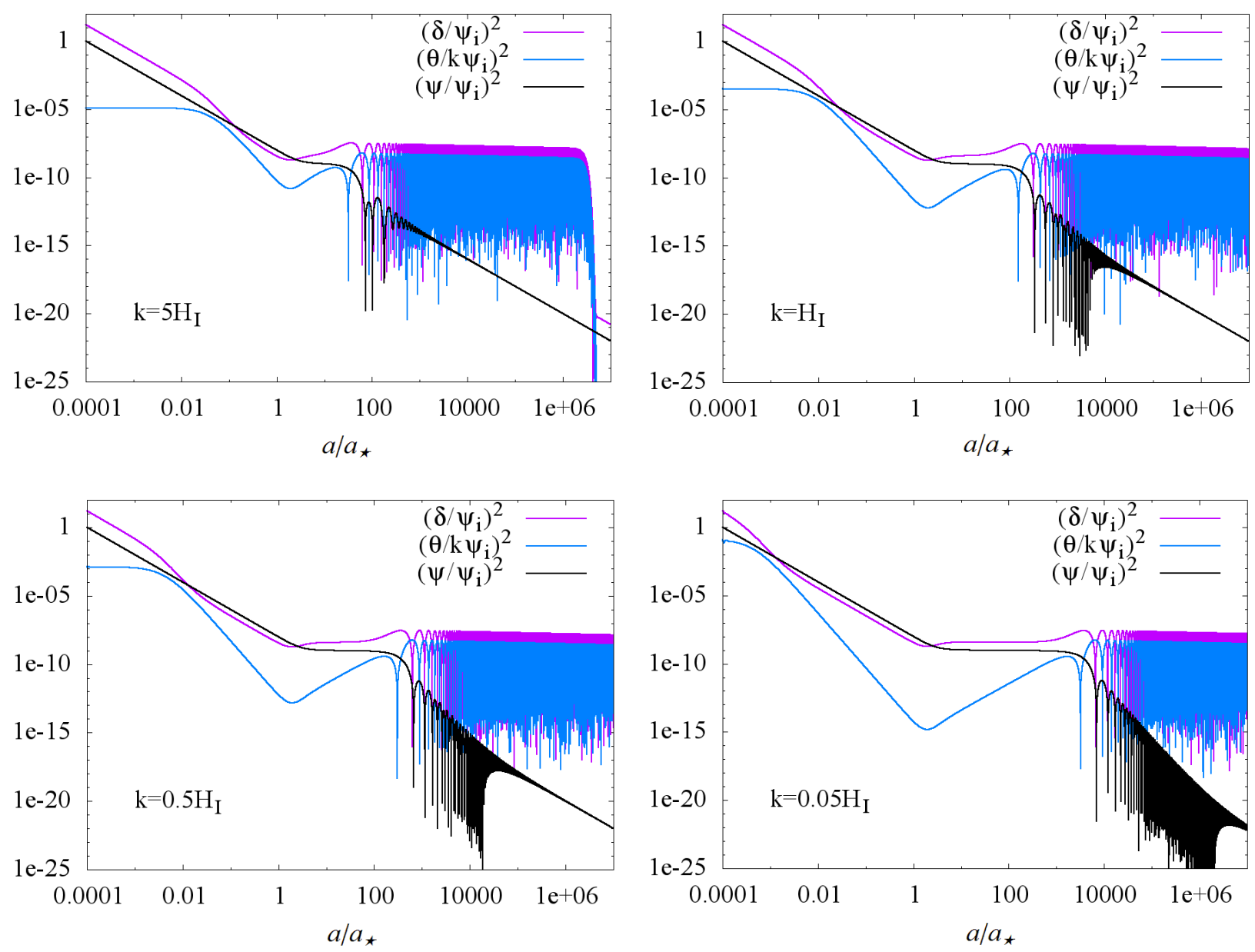

Figura 6.7: Evolução das perturbações escalares durante a deflação.

Mostra-se a evolução das perturbações escalares em função do fator de escala durante os períodos inflacionário e de domínio da radiação, para várias escalas que inicialmente estavam dentro do horizonte, mas que saem do horizonte no transcurso do período inflacionário.

\subsubsection{Escala pivô}

O índice espectral das perturbações escalares $n_{s}$, que varia com o número de onda $k$, é medido em uma escala específica. No caso do satélite Planck [Planck Collaboration, 2014], ele é medido na escala $k_{p}=0.05 \mathrm{Mpc}^{-1}$, com o que, a amplitude do espectro de potências primordial para as perturbações escalares se escreve como:

$$
P(k)=A_{s}\left(\frac{k}{k_{p}}\right)^{n_{s}-1+\frac{1}{2} \frac{d n_{s}}{d \ln k} \ln \left(\frac{k}{k_{p}}\right)+\frac{1}{6} \frac{d^{2} n_{s}}{d \ln k^{2}} \ln ^{2}\left(\frac{k}{k_{p}}\right),}
$$


Se quisermos comparar a predição do modelo deflacionário para o índice espectral escalar com os resultados do satélite Planck, devemos avaliar nossas expressões no instante em que a escala $k_{p}$ sai do horizonte de Hubble durante o período primordial acelerado.

No caso em que $v=0$ podemos expressar a densidade de energia da radiação como:

$$
\rho(a)=\rho_{G U T} \frac{\left(a / a_{\star}\right)^{2 n}}{\left[1+\left(a / a_{\star}\right)^{2 n}\right]^{1+2 / n}} \stackrel{a \gg a_{\star}}{\longrightarrow} \rho_{G U T}\left(\frac{a_{\star}}{a}\right)^{4} \equiv \rho_{c r}^{0} \Omega_{\gamma}^{0}\left(\frac{a_{0}}{a}\right)^{4},
$$

e dado que $H_{G U T} \approx H_{0} \times 10^{53}$ teremos que $a_{\star} \approx \Omega_{\gamma}^{1 / 4} \times 10^{-53 / 2}$. Já no instante em que a escala pivô sai do horizonte de Hubble bem no começo do período primordial acelerado teremos que

$$
H(a)=\frac{H_{G U T}}{\left[1+\left(a / a_{\star}\right)^{2 n}\right]^{1 / n}} \stackrel{a \ll a_{\star}}{\longrightarrow} H_{G U T},
$$

com o que

$$
a_{p} \approx H_{G U T}^{-1} k_{p} \approx 1.24 a_{\star} \times 10^{-31}
$$

\subsection{UNIVERSO RECENTE}

Agora vamos nos concentrar em estudar a solução às equações de movimento (6.3) para o universo recente composto pelo fluido material e pelo vácuo na aproximação $H \ll H_{I}$, sobre as hipóteses de homogeneidade e isotropia em grandes escalas. Neste caso a equação para a evolução do parâmetro de Hubble (6.3) pode ser aproximada como:

$$
a H H^{\prime}+\xi H^{2}-\frac{(1+\omega)}{2} \Lambda_{c}=0
$$

onde mais uma vez usamos a notação $\xi \equiv 3(1+\omega)(1-v) / 2$. A primeira integral desta equação produz a expressão:

$$
H^{2}=\frac{\Lambda_{c}}{3(1-v)}\left\{\left(\frac{C_{1}}{a}\right)^{2 \xi}+1\right\}
$$

onde a constante de integração

$$
C_{1}^{2 \xi}=a_{0}^{2 \xi}\left(\frac{3 H_{0}^{2}(1-v)}{\Lambda_{c}}-1\right)
$$

foi obtida impondo a condição de fronteira $H\left(a_{0}\right) \equiv H_{0}$ hoje. Usando a solução acima, as equações de Friedmann nos permitem obter as densidades de energia:

$$
8 \pi G \rho_{T}(a)=\frac{\Lambda_{c}}{1-v}\left\{\left(\frac{C_{1}}{a}\right)^{2 \xi}+1\right\}, \quad 8 \pi G \rho(a)=\Lambda_{c}\left(\frac{C_{1}}{a}\right)^{2 \xi}
$$

Se agora exigirmos que $8 \pi G \rho=3 H_{0}^{2} \Omega$, obteremos que

$$
\Lambda_{c}=3 H_{0}^{2}\left(\Omega_{\Lambda}^{0}-v\right), \quad \rightarrow \quad C_{1}=a_{0}\left(\frac{1-\Omega_{\Lambda}^{0}}{\Omega_{\Lambda}^{0}-v}\right)^{1 / 2 \xi},
$$


com o que podemos reescrever o parâmetro de Hubble (6.47) como

$$
H^{2}(a)=\frac{H_{0}^{2}}{1-v}\left\{\Omega_{X}^{0}\left(\frac{a}{a_{0}}\right)^{-2 \xi}+\Omega_{\Lambda}^{0}-v\right\}
$$

onde usamos a condição de planeza do universo $\Omega_{X}^{0}+\Omega_{\Lambda}^{0}=1$. Também a densidade de energia do fluido material pode ser reescrita como

$$
\rho(a)=\rho^{0}\left(\frac{a}{a_{0}}\right)^{-2 \xi}
$$

onde $\rho^{0}$ é o seu valor hoje. Na expressão anterior podemos ver que para $v=0$ se recupera a expressão padrão para evolução da densidade de energia de um fluido material livre $\rho=\rho^{0}\left(a / a_{0}\right)^{-3(1+\omega)}$. A correção do parâmetro $v$ presente no modelo que estamos analisando vem da troca de energia entre o vácuo e o campo material. Similarmente, devido a dita interação, a densidade do vácuo evolui como

$$
\rho_{\Lambda}(a)=\rho_{\Lambda}^{0}+\frac{v \rho^{0}}{1-v}\left\{\left(\frac{a}{a_{0}}\right)^{-2 \xi}-1\right\}
$$

e no limite em que $v \rightarrow 0$ se recupera $\Lambda=3 H_{0}^{2} \Omega_{\Lambda}^{0}=$ cte e $\rho_{\Lambda}(a)=\rho_{\Lambda}^{0}=$ cte, como é o caso do modelo $\Lambda$ CDM. Como complemento, é possível verificar que as equações (6.52) e (6.53) satisfazem a equação de conservação local da energia total (2.34), que pode ser escrita em função do fator de escala como

$$
\rho_{\Lambda}^{\prime}(a)+\rho^{\prime}(a)+\frac{3}{a}(1+\omega) \rho(a)=0
$$

Adicionalmente, podemos integrar a equação (6.47) na variável temporal para obter

$$
a(t)=a_{0}\left(\frac{1-\Omega_{\Lambda}^{0}}{\Omega_{\Lambda}^{0}-v}\right)^{1 / 2 \xi} \sinh ^{1 / \xi}\left[\xi\left(\frac{\Omega_{\Lambda}^{0}-v}{1-v}\right)^{1 / 2} H_{0}\left(t-t_{0}\right)+C_{2}\right]
$$

onde a constante de integração $C_{2}$ pode ser obtida impondo a condição de fronteira $a\left(t_{0}\right) \equiv a_{0}$, dando como resultado:

$$
C_{2} \equiv \operatorname{ArcSinh}\left[\left(\frac{\Omega_{\Lambda}^{0}-v}{1-\Omega_{\Lambda}^{0}}\right)^{1 / 2}\right]
$$

Eventualmente, pode-se usar a solução (6.55) para expressar as densidades de energia do fluido material (6.52) e do vácuo (6.53) em função do tempo cósmico $\rho=\rho(t)$ e $\Lambda=\Lambda(t)$.

\subsubsection{Domínio da matéria e do vácuo remanescente}

Nesta seção vamos considerar a expansão do universo muito depois do período inflacionário e da era de domínio da radiação, depois da recombinação, quando o universo pode ser aproximado como sendo composto pela mistura de poeira $(\omega=0)$ e do vácuo descrito pela expressão (6.2) com $H \ll H_{I}$. Neste caso os termos proporcionais a $H^{\alpha}, \operatorname{com} \alpha>2$ são completamente desprezíveis se comparados com o termo proporcional a $H^{2}$, com o que a expressão para a densidade do vácuo se reduz a:

$$
\Lambda(H)=\Lambda_{0}+3 v\left(H^{2}-H_{0}^{2}\right)
$$


onde $\Lambda_{0} \equiv \Lambda_{c}+3 v H_{0}^{2}$ é o valor atual para a densidade do vácuo. É claro que $\Lambda_{c}$ tem um papel importante no valor atual da densidade do vácuo, mas ainda temos a dependência em $H^{2}$ que promove uma dinâmica ainda hoje. Devido a esta dinâmica podemos ajustar o parâmetro $v$ comparando o modelo com as observações. Fazendo uma análise conjunta dos dados de supernovas Ia, do parâmetro de deslocamento do primeiro pico do espectro de potências das anisotropias na radiação cósmica de fundo, e das oscilações acústicas dos bários para um universo plano é possível ajustar os parâmetros $\Omega_{m}^{0} \simeq 0.27-0.28$ e $|v|=\mathcal{O}\left(10^{-3}\right)$ [Grande et al., 2011; Basilakos et al., 2009, 2012].

Para um universo recente $H \ll H_{I}$ composto por vácuo e poeira, a equação que descreve a evolução do parâmetro de Hubble (6.3) possui a solução dada pela expressão (6.51) com $\omega=0$ :

$$
H^{2}(a)=\frac{H_{0}^{2}}{1-v}\left\{\left(1-\Omega_{\Lambda}^{0}\right)\left(\frac{a}{a_{0}}\right)^{-3(1-v)}+\Omega_{\Lambda}^{0}-v\right\} .
$$

Similarmente, as densidades de energia da matéria e do vácuo ficam expressas como:

$$
\rho_{m}(a)=\rho_{m}^{0}\left(\frac{a}{a_{0}}\right)^{-3(1-v)}, \quad \rho_{\Lambda}(a)=\rho_{\Lambda}^{0}+\frac{v \rho_{m}^{0}}{1-v}\left\{\left(\frac{a}{a_{0}}\right)^{-3(1-v)}-1\right\}
$$

onde $\rho_{m}^{0}$ e $\rho_{\Lambda}^{0}$ são os valores para as densidades de energia da matéria e do vácuo hoje.

Integrado mais uma vez a equação (6.57) em relação ao tempo cósmico obtemos a seguinte dependência temporal para o fator de escala:

$$
a(t)=a_{0}\left(\frac{1-\Omega_{\Lambda}^{0}}{\Omega_{\Lambda}^{0}-v}\right)^{\frac{1}{3(1-v)}} \sinh ^{\frac{2}{3(1-v)}}\left[3 H_{0} \sqrt{(1-v)\left(\Omega_{\Lambda 0}-v\right)}\left(t-t_{0}\right) / 2+C_{2}\right] .
$$

Fica claro nas expressões anteriores que para $v=0$ se recuperam as soluções do modelo $\Lambda$ CDM para o fator de escala, para a evolução da densidade de energia da matéria, como também se reobtém uma energia do vácuo constante $\rho_{\Lambda}=\rho_{\Lambda}^{0}$ (ou $\Lambda=\Lambda_{0}$ ). É de ressaltar que com base nos ajustes de $|v| \leq \mathcal{O}\left(10^{-3}\right)$ obtidos confrontando o modelo com os dados cosmológicos [Shapiro et al., 2003; Basilakos et al., 2009; Grande et al., 2011], podemos ver que o modelo defere muito pouco do modelo padrão para um universo recente. Extrapolando a solução para o futuro distante obtemos um universo dominado por uma densidade do vácuo efetivamente constante, $H \approx H_{0} \sqrt{\left(\Omega_{\Lambda}^{0}-v\right) /(1-v)}$, ver equação $(6.57)$ para $a \gg a_{0}$, o que implica uma era de Sitter final para a evolução do universo.

\section{Parâmetro de desaceleração no universo recente}

Na época em consideração temos que $\omega=0$ e $H / H_{I} \ll 1$. Assim, com ajuda das equações (6.5) e (6.57), o parâmetro de desaceleração assume a forma

$$
q(a)=\xi\left\{1+\left(\frac{a}{a_{0}}\right)^{2 \xi}\left(\frac{\Omega_{\Lambda}^{0}-v}{1-\Omega_{\Lambda}^{0}}\right)\right\}^{-1}-1 \quad \stackrel{\omega \rightarrow 0}{\longrightarrow} \quad \frac{(1-3 v) \Omega_{m}^{0}\left(a / a_{0}\right)^{-3(1-v)}-2\left(\Omega_{\Lambda}^{0}-v\right)}{2 \Omega_{m}^{0}\left(a / a_{0}\right)^{-3(1-v)}+2\left(\Omega_{\Lambda}^{0}-v\right)}
$$

No limite $v \rightarrow 0$, esta expressão se reduz à predição do modelo padrão. Em particular, o valor atual do parâmetro de desaceleração é $q_{0}=\left(3 \Omega_{m}^{0}-2\right) / 2 \simeq-0.58$, para $\Omega_{m}^{0} \simeq 0.28$. A transição do período 


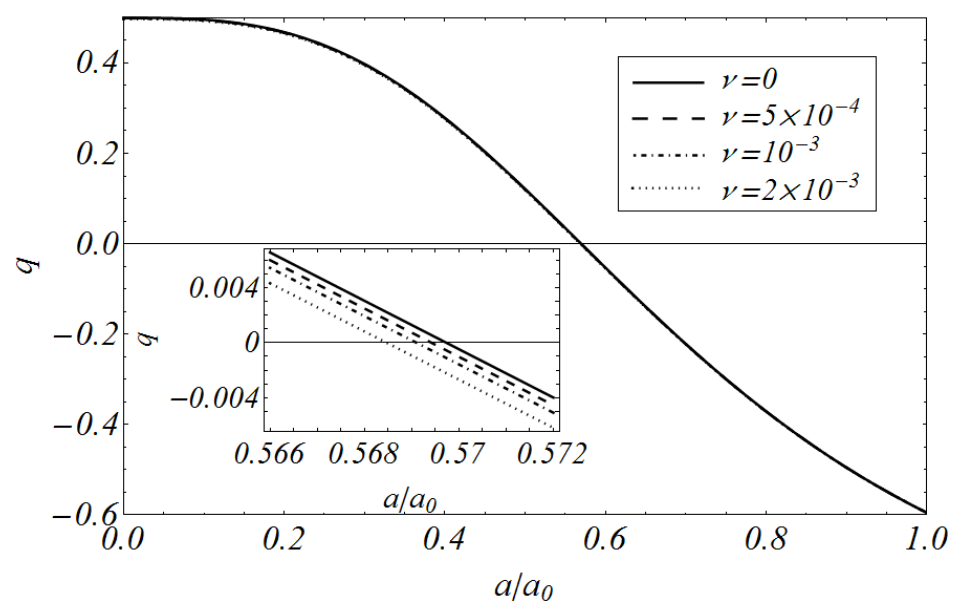

Figura 6.8: Parâmetro de desaceleração na era recente para o modelo $\Lambda=\Lambda_{c}+3 v H^{2}$.

Podemos ver que para valores pequenos de $v$ o parâmetro de desaceleração difere pouco do predito pelo modelo $\Lambda \mathrm{CDM}$, onde $v=0$. Mesmo assim, para valores maiores de $v$ a transição do período dominado por poeira $(q \approx 1 / 2)$ para o período dominado pelo vácuo remanente acontece mais cedo.

desacelerado, dominado pela matéria, para o regime acelerado, dominado pela densidade remanescente do vácuo ocorre quando

$$
a_{l t}=a_{0}\left\{\frac{\Omega^{0}(\xi-1)}{\Omega_{\Lambda}^{0}-v}\right\}^{1 / 2 \xi} \stackrel{\omega \rightarrow 0}{\longrightarrow}\left\{\frac{\Omega_{m}^{0}(1-3 v)}{2\left(\Omega_{\Lambda}^{0}-v\right)}\right\}^{1 / 3(1-v)},
$$

e para $v \rightarrow 0$ é igual a $a_{l t} \simeq 0.58$.

Embora a natureza dinâmica da densidade do vácuo (6.56), para tempos recentes é importante entender que um modelo do tipo $\Lambda=\sum_{n} c_{n} H^{n}$ com $\Lambda_{c}=0$ não se ajusta bem às observações cosmológicas [Basilakos et al., 2009] já que para $\Lambda_{c}=0$ o parâmetro de desaceleração não muda de sinal, produzindo um universo (durante a era da matéria e posterior) sempre acelerado ou sempre desacelerado.

No caso que estamos estudando $\left(\Lambda \approx \Lambda_{c}+c_{2} H^{2}\right)$ a condição $\Lambda_{c}=0$ faria com que a equação (6.57) assuma a forma $H^{2}(a)=H_{0}^{2}\left(a / a_{0}\right)^{-3(1-v)}$, e por sua parte o parâmetro de desaceleração seria

$$
q=-1-a \frac{H^{\prime}(a)}{H(a)}=-1+\frac{3}{2}(1-v)=c t e,
$$

que dá como resultado que o universo é acelerado hoje somente se $v>1 / 3$, sendo incompatível com os resultados obtidos por [Shapiro et al., 2003; España-Bonet et al., 2004; Opher and Pelinson, 2004; Alcaniz and Lima, 2005; Opher and Pelinson, 2005; Wang and Meng, 2005; Fabris et al., 2007; Costa et al., 2008; Jesus et al., 2008; Basilakos et al., 2009, 2010; Grande et al., 2011; Basilakos et al., 2012] para $|v| \ll 1$. Além disso $q$ apresentaria um valor constante, ou seja, o universo deveria ter sido acelerado a partir do fim da era da radiação até hoje, sendo incompatível com os dados prevenientes de supernovas.

Depois de ressaltar a importância de ter um termo constante $\Lambda_{c} \neq 0$ na densidade do vácuo (6.2) na hora de ajustar as observações do universo recente, vale a pena apresentar uma justificativa natural 
para a presença de dito termo constante: a integração das equações do grupo de renormalização (5.14) devem introduzir necessariamente uma constante aditiva na estrutura funcional para $\rho_{\Lambda}(H)$.

\subsubsection{Aliviando os problemas da coincidência e da constante cosmológica}

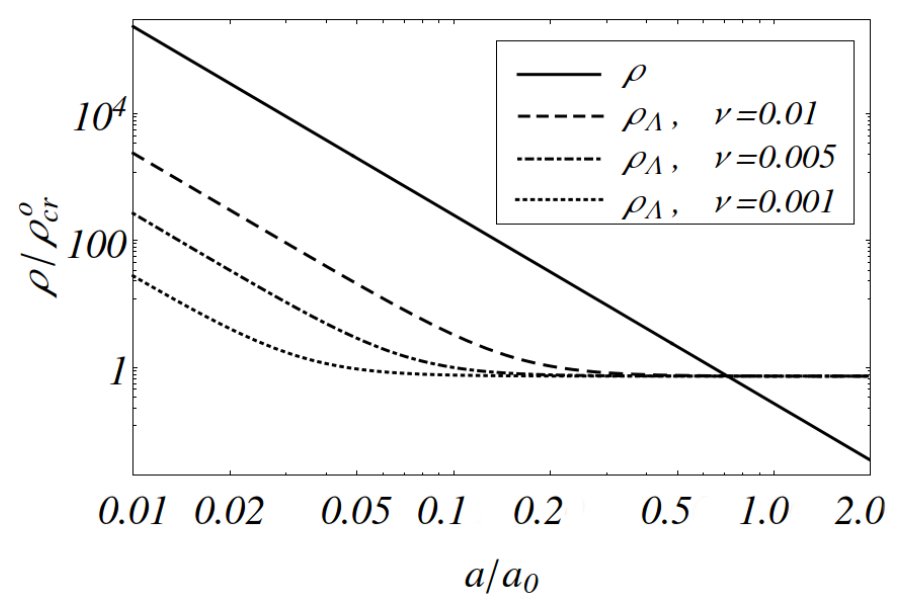

Figura 6.9: Evolução das densidade no universo recente para o modelo com $\Lambda=\Lambda_{c}+3 v H^{2}$. Esta figura mostra como as densidades do vácuo e da matéria evoluem de forma similar durante o período de expansão desacelerada, aliviando o problema da coincidência. Analiticamente, tem-se que $\rho_{\Lambda} / \rho \approx v /(1-v)$ antes do domínio do vácuo remanescente.

Embora o presente modelo provê uma redução drástica na densidade do vácuo (capaz de produzir radiação suficiente para sair do período inflacionário sem precisar de um reaquecimento posterior, e ainda decrescendo durante as eras de domínio dos fluidos materiais) o seu valor não pode ser predito pelo modelo, mas sim, deve ser extraído das observações obtendo que $\Lambda_{c}=8 \pi G \rho_{\Lambda}^{0}-3 v H_{0}^{2}$.

Mesmo assim, o modelo faz mais ameno o problema que surge ao supor que o pequeno valor observado hoje para a densidade do vácuo é uma quantidade imutável (assumido pelo modelo padrão durante toda a história do universo) dando a esta densidade um caráter dinâmico que permite a sua dramática redução a partir do valor predito pela física de partículas - c capaz de produzir a inflação- até atingir o pequeno valor observado hoje. Adicionalmente, ao invés de outros modelos, onde o período inflacionário e o período de aceleração atual estão totalmente desconectados, no presente modelo eles estão naturalmente correlacionados.

Agora esses dois estágios podem ser pensados como duas eras conectadas por uma única dinâmica que permite um período intermediário dominado pela radiação e pela matéria. A tradicional inabilidade de compreender porque $\rho_{\Lambda}^{0} \ll \rho_{\Lambda}\left(H_{I}\right)$ (o velho problema da constante cosmológica) é transformada em um problema que se reduz simplesmente a entender porque $\rho_{\Lambda}^{0}$ possui precisamente o valor observado hoje, entendendo já que ele deve ser uma quantidade muito menor que o valor esperado do vácuo em altas energias.

Adicionalmente, a lei de conservação covariante do tensor momento energia total, contida nas equações de Einstein, obriga que a densidade do vácuo dinâmica seja transferida para as componentes materiais. 
Graças a isso, no modelo que estamos considerando no presente trabalho, durante os períodos de domínio da matéria e do vácuo remanescente, as suas densidades estão relacionadas pelas expressões (6.58):

$$
\left(\rho_{\Lambda}(t)-\rho_{\Lambda}^{0}\right)(1-v)=v\left(\rho_{m}(t)-\rho_{m}^{0}\right)
$$

aliviando o problema da coincidência. Em particular, durante todo o período de domínio da matéria, podemos relacionar as densidades da matéria e do vácuo como:

$$
\rho_{\Lambda}(t) \approx \frac{v}{1-v} \rho_{m}(t)
$$

Esse comportamento é mostrado na figura 6.9, onde podemos ver que as duas densidades evoluem aproximadamente com a mesma lei durante a era de domínio da matéria.

\subsection{AJUSTANDO O PARÂMETRO $v$}

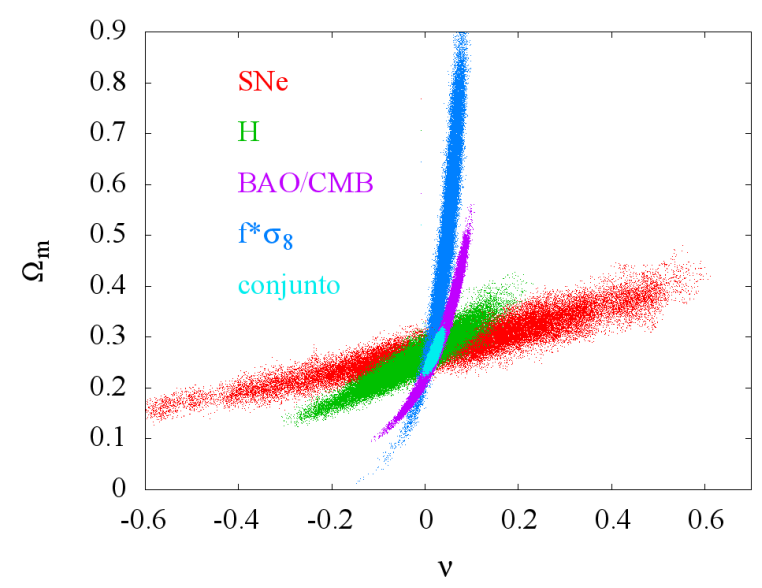

Figura 6.10: Quebra da degenerescência do sistema no espaço dos parâmetros $v$ e $\Omega_{m}$. Esta distribuição foi gerada implementando a análise separada para cada observável, deixando fixos $H_{0}$ e $\sigma_{8}$ iguais a seus valores padrão.

Para ajustar o parâmetro livre $v$ durante os períodos recentes do universo vamos fazer uma análise conjunta usando dados de (i) Supernovas [Suzuki et al., 2012], (ii) da razão entre a posição do primeiro pico do espectro de potências das anisotropias na radiação cósmica de fundo e a escala de dilatação das oscilações acústicas dos bárions $D_{V}$ [WMAP], (iii) do parâmetro de Hubble como para vários fatores de escala [Farooq and Ratra, 2013], (iv) e da função de crescimento multiplicado pela amplitude do espectro de potências linear da matéria na escala de $8 \mathrm{Mpc} / h, \sigma_{8}$, ver [Ramos et al., 2014].

\section{Supernovas}

Usando os dados das magnitudes das supernovas, da compilação Union2.1 [Suzuki et al., 2012]:

$$
m(z) \equiv 5 \log \left(H_{0} d_{L}\right)+M
$$


onde $d_{L}$ é a distância de luminosidade física definida por:

$$
d_{L}=(1+z) D(z), \quad D(z) \equiv \int_{0}^{z} \frac{d z^{\prime}}{H\left(z^{\prime}\right)} .
$$

Definindo agora a magnitude $m_{T M}$ como sendo

$$
m_{T M}(z) \equiv m(z)-M
$$

podemos calcular o $\chi^{2}$ na forma

$$
\chi^{2}=\sum_{i=1}^{N} \frac{\left(m_{o b s}^{i}-\left(m_{T H}\left(z_{i}\right)+M\right)\right)^{2}}{\left(\delta m^{i}\right)^{2}}
$$

onde $\delta m^{i}$ é a incerteza da magnitude observada $m_{o b s}^{i}$. Derivando esta expressão em relação à constante $M$, e igualando o resultado a zero, podemos escrever em evidência o valor de $M$ onde o $\chi^{2}$ é máximo:

$$
\frac{\partial \chi^{2}}{\partial M}=0, \quad M=\left(\sum_{i} \frac{m_{o b s}^{i}-m_{T M}^{i}}{\left(\delta m^{i}\right)^{2}}\right)\left(\sum_{i} \frac{1}{\left(\delta m^{i}\right)^{2}}\right)^{-1}
$$

Introduzindo este valor calculado para $M$ na expressão (6.67) podemos obter o resultado apresentado em cor verde no painel esquerdo do gráfico 6.10, onde podemos observar uma grande degenerescência entre os parâmetros $v$ e $\Omega_{m}$, em relação aos dados de supernova.

\section{Dados de $B A O / C M B$}

Para quebrar esta degenerescência foi usado por [Costa et al., 2012] a quantidade:

$$
f_{z} \equiv \frac{d_{A}\left(z_{*}\right)}{D_{V}(z)}
$$

que foi construída, para que independa de $H_{0}$, usando:

- a posição do primeiro pico do espectro de potências da radiação, que representa a escala angular do horizonte de som na época da recombinação [Komatsu et al., 2011]:

$$
\ell_{A} \equiv \frac{\pi d_{A}\left(z_{*}\right)}{r_{S}\left(z_{*}\right)}=302.09 \pm 0.76
$$

onde $d_{A}$ é a distância diametral angular comóvel até a época da recombinação, $z_{*}=1091.30 \pm$ 0.91 [Komatsu et al., 2011],

$$
d_{A}(z) \equiv \frac{f_{k}\left(H_{0} \sqrt{\left|\Omega_{k}\right|} D(z)\right)}{H_{0} \sqrt{\left|\Omega_{k}\right|}}, \quad f_{k}(x) \equiv \begin{cases}\sin (x), & \Omega_{\kappa}<0(\kappa=1) \\ x, & \Omega_{\kappa}=0(\kappa=0) \\ \sinh (x), & \Omega_{\kappa}>0(\kappa=-1)\end{cases}
$$

$r_{\mathcal{S}}\left(z_{*}\right)$ é o horizonte de som comóvel dos fótons, também durante o desacoplamento:

$$
r_{s} \equiv \int_{z_{*}}^{\infty} \frac{c_{s}}{H(z)} d z, \quad c_{s}^{2}=\frac{1}{3(1+R)}
$$


$c_{s}$ é a velocidade do som do plasma bárions-fótons, e $R$ é a razão entre a densidade de energia de bárions e fótons $R \equiv 3 \rho_{b} / 4 \rho_{\gamma}$.

- e a razão da escala do horizonte durante a drag epoch $r_{s}\left(z_{d}\right)$, e a escala de dilatação do BAO (oscilações acústicas dos bárions) $D_{V}\left(z_{B A O}\right)$ definida como

$$
D_{V}(z) \equiv\left[\frac{z d_{A}^{2}(z)}{H(z)}\right]^{1 / 3},
$$

onde $z_{d}$ foi ajustado numericamente por [Eisenstein and Hu, 1998] usando simulações da recombinação:

$$
\begin{aligned}
& z_{d}=1291 \frac{\left(\Omega_{m} h^{2}\right)^{0.251}}{1+0.659\left(\Omega_{m} h^{2}\right)^{0.828}}\left(1+b_{1}\left(\Omega_{m} h^{2}\right)^{b_{2}}\right), \\
& b_{1}=0.313\left(\Omega_{m} h^{2}\right)^{-0.491}\left(1+0.607\left(\Omega_{m} h^{2}\right)^{0.674}\right), \\
& b_{2}=0.238\left(\Omega_{m} h^{2}\right)^{0.223} .
\end{aligned}
$$

De forma similar ao feito por [Costa et al., 2012; Ramos et al., 2014] (usando os valores reportados por [Sollerman et al., 2009; Percival et al., 2010; Blake, 2011; Griostri et al., 2012; Anderson et al., 2012]) usaremos os valores atualizados por [WMAP] para restringir os parâmetros livres do presente modelo.

\subsubsection{Parâmetro de Hubble}

No caso do parâmetro de Hubble, como foi feito por [Ramos et al., 2014], vamos usar os dados observacionais informados por [Farooq and Ratra, 2013] na análise conjunta, com o objetivo de ajustar os parâmetros livres dos modelo deflacionário. Neste caso, usaremos a expressão teórica dada pela solução (6.57), desconsiderando qualquer contribuição da radiação, devido ao baixo deslocamento para o vermelho dos dados com os que estamos trabalhando.

\subsubsection{Função de crescimento das perturbações lineares}

No caso de um universo dominado por uma mistura de matéria não relativística e energia escura (que não estamos interessados em perturbar no presente trabalho), e em ausência de estresse anisotrópico, a componente espacial da equação de Einstein é:

$$
\ddot{\phi}+3 \mathcal{H} \dot{\phi}+\left(2 \dot{\mathcal{H}}+\mathcal{H}^{2}\right) \phi=0
$$

onde usamos $\sigma=0(\phi=\psi)$, e $\omega=\delta \mathcal{P}=0$. Usando as equações de Einstein a ordem zero, podemos reescrever a equação diferencial acima como sendo:

$$
a^{2} \rho_{T} \phi^{\prime \prime}+\frac{7 \rho_{T}-3 \mathcal{P}_{T}}{2} a \phi^{\prime}-3 \mathcal{P}_{T} \phi=0
$$


e usando a solução (6.57-6.58) pode ser re-escrita como:

$$
a^{2} \phi^{\prime \prime}\left(\Omega_{m} \chi+1-\Omega_{m}-v\right)+\left(\frac{7+3 v}{2} \Omega_{m} \chi+5\left(1-\Omega_{m}-v\right)\right) a \phi^{\prime}+3\left(v \Omega_{m} \chi+1-\Omega_{m}-v\right) \phi=0 .
$$

Para encontrar as condições iniciais, com as que deve ser integrada esta equação, tomamos o limite $a \ll 1(\chi \ll 1)$ obtendo:

$$
a^{2} \phi^{\prime \prime}+\frac{7+3 v}{2} a \phi^{\prime}+3 v \phi=0
$$

que tem como solução

$$
\phi=c_{1} a^{-(5+3 v-\sqrt{25-9 v(2-v)}) / 4}+c_{2} a^{-(5+3 v+\sqrt{25-9 v(2-v)}) / 4} .
$$

O segundo termo desta solução decresce rapidamente com o fator de escala (no limite de $v \rightarrow 0$ decresce como $a^{-5 / 2}$ ) enquanto que o primeiro termo decresce muito pouco (no limite de $v \rightarrow 0$ permanece constante), pelo que a condição inicial adequada para integrar a equação (6.77) é:

$$
\phi=\phi_{i}, \quad \phi^{\prime}=\frac{c_{3}}{a} \phi_{i}, \quad c_{3} \equiv-(5+3 v-\sqrt{25-9 v(2-v)}) / 4 .
$$

Integrando numericamente a equação (6.77) usando as condições iniciais (6.80) podemos obter a evolução temporal da perturbação métrica $\phi$. Como nosso interesse radica na perturbação $\delta$, podemos usar a componente temporal da equação de Einstein:

$$
-4 \pi G a^{2} \rho \delta=k^{2} \phi+3 \mathcal{H}^{2}\left(a \phi^{\prime}+\phi\right)
$$

usando a densidade de energia dada pela solução (6.58) e para escalas dento do horizonte $(k \gg \mathcal{H})$ obtemos:

$$
\delta=-\frac{2 k^{2}}{3 H_{0}^{2} \Omega_{m}} a^{1-3 v} \phi
$$

com o que o observável

$$
\left(f \times \sigma_{8}\right)(z) \equiv \frac{\partial \ln \delta}{\partial \ln a} \times \sigma_{8} \frac{\delta}{\delta_{0}}=\sigma_{8} a \frac{\delta^{\prime}}{\delta_{0}}=a^{1-3 v}\left(\phi+(1-3 v) a \phi^{\prime}\right) \frac{\sigma_{8}}{\phi_{0}},
$$

fica expresso em função de $\phi$, que por sua vez é dado pela solução da equação diferencial (6.77) com as condições iniciais (6.80). Esta expressão teórica será comparada, como foi feito em [Ramos et al., 2014], com os dados informados por [Beutler et al., 2012; Percival et al., 2004; Blake, 2011; Samushina et al., 2012; Ried et al., 2012; Torre et al., 2013].

Usando um observável se obtém uma grande degenerescência no espaço dos parâmetros $v$ e $\Omega_{m}$ (ver figura 6.10, cor vermelha). Esta degenerescência pode ser quebrada fazendo uma análise conjunta de todos os observáveis, SN, BAO/CMB, função de crescimento e $H(z)$. O resultado da análise conjunta produz uma restrição para os parâmetros $v$ e $\Omega_{m}$ (região azul na figura 6.10). 


\subsection{RESULTADOS}

Inicialmente, usando a análise separada de cada um dos observáveis foi obtida a distribuição mostrada na figura 6.10 para os parâmetros $\Omega_{m}$ e $v$. Esta figura mostra a grande degenerescência quando se faz a analise separa de cada conjunto de dados observacionais.

Como caso particular, na figura 6.10, podemos ver que os dados de Supernovas e H(a) possuem a mesma classe de degenerescência em relação ao parâmetro $v$, enquanto que os dados de BAO/CMB e da função de crescimento são altamente degenerados na variável $\Omega_{m}$, mas felizmente o primeiro par de observáveis quebra a degenerescência do segundo, gerando um bom ajuste para os dois parâmetros.

Deixando livres os parâmetros $H_{0}, \sigma_{8}, \Omega_{m}$ e $v$ foram comparadas as predições teóricas do modelo com os dados de Supernovas, BAO/CMB, H(z) e da função de crescimento, mediante uma cadena de Montecarlo Metropolis et al. [1953] de 50000 pontos obtendo a tabela 1, que informa os parâmetros ajustados.

\begin{tabular}{|c|c|c|c|c|c|c|}
\hline Modelo Padrão & Média & Desvío da M. & Melh. Ajuste & Inc. (68.3\%) & Inc. (95.4\%) & Inc. (99.7\%) \\
\hline \hline$\Omega_{m}$ & 0.233 & \pm 0.009 & 0.233 & ${ }_{-0.010}^{+0.008}$ & ${ }_{-0.018}^{+0.016}$ & ${ }_{-0.026}^{+0.023}$ \\
\hline$H_{0}$ & 71.7 & ${ }_{-1.7}^{+1.5}$ & 71.8 & ${ }_{-1.5}^{+1.6}$ & ${ }_{-3.0}^{+3.0}$ & ${ }_{-4.4}^{+4.5}$ \\
\hline$\sigma_{8}$ & 0.804 & ${ }_{-0.034}^{+0.033}$ & 0.804 & ${ }_{-0.033}^{+0.035}$ & ${ }_{-0.063}^{+0.065}$ & ${ }_{-0.083}^{+0.091}$ \\
\hline
\end{tabular}

\begin{tabular}{|c|c|c|c|c|c|c|}
\hline Deflacionário & Média & Desvio da M. & Melh. Ajuste & Inc. (68.3\%) & Inc. (95.4\%) & Inc. (99.7\%) \\
\hline \hline$\Omega_{m}$ & 0.285 & ${ }_{-0.021}^{+0.022}$ & 0.285 & ${ }_{-0.021}^{+0.022}$ & ${ }_{-0.043}^{+0.042}$ & ${ }_{-0.066}^{+0.058}$ \\
\hline$H_{0}$ & 69.2 & ${ }_{-1.8}^{+1.7}$ & 69.1 & ${ }_{-1.8}^{+1.6}$ & ${ }_{-3.5}^{+3.2}$ & ${ }_{-5.3}^{+4.8}$ \\
\hline$\sigma_{8}$ & 0.873 & ${ }_{-0.044}^{+0.045}$ & 0.877 & ${ }_{-0.041}^{+0.049}$ & ${ }_{-0.085}^{+0.088}$ & ${ }_{-0.13}^{+0.12}$ \\
\hline$v$ & 0.030 & \pm 0.010 & 0.030 & ${ }_{-0.009}^{+0.011}$ & ${ }_{-0.018}^{+0.021}$ & ${ }_{-0.027}^{+0.031}$ \\
\hline
\end{tabular}

Tabela 1: Resultado da análise conjunta dos dados de Supernovas, BAO/CMB, H(z) e função de crescimento usando uma cadeia de Montecarlo de 50000 pontos. Todos os resultados são compatíveis com o modelo padrão em um margem de $2 \sigma$, a excepção do parâmetro $v$, que difere do modelo padrão $(v=0)$ em $3 \sigma$, e com um cociente entre as evidências do modelo padrão e do modelo deflacionário igual a 1.0124 .

Como complemento mostra-se na figura 6.12 a comparação entre o ajuste teórico do modelo padrão (curvas azuis) com o melhor ajuste obtido no presente trabalho (curvas vermelhas).

\subsection{RESUMO DO CAPÍTULO}

Neste capítulo, estudamos um modelo dinâmico do vácuo proposto fenomenologicamente [Perico et al., 2013a], que provê uma historia cósmica completa. A expressão dinâmica $\Lambda=\Lambda(H)$ estudada consiste em uma serie de potências em função do parâmetro de Hubble, onde o termo constante é responsável pelo comportamento para baixas energias, o termo proporcional a $H^{2}$ é responsável pela dinâmica durante os períodos de domínio dos fluidos materiais -ainda hoje introduz correções ao termo constantee o termo proporcional a $H^{4}$ é o primeiro termo relevante responsável pelo período inflacionário.

Este tipo de modelos prediz que o universo possui um estado não singular de expansão acelerada. A duração do período acelerado pode ser arbitrariamente longa quanto menor for a densidade inicial 

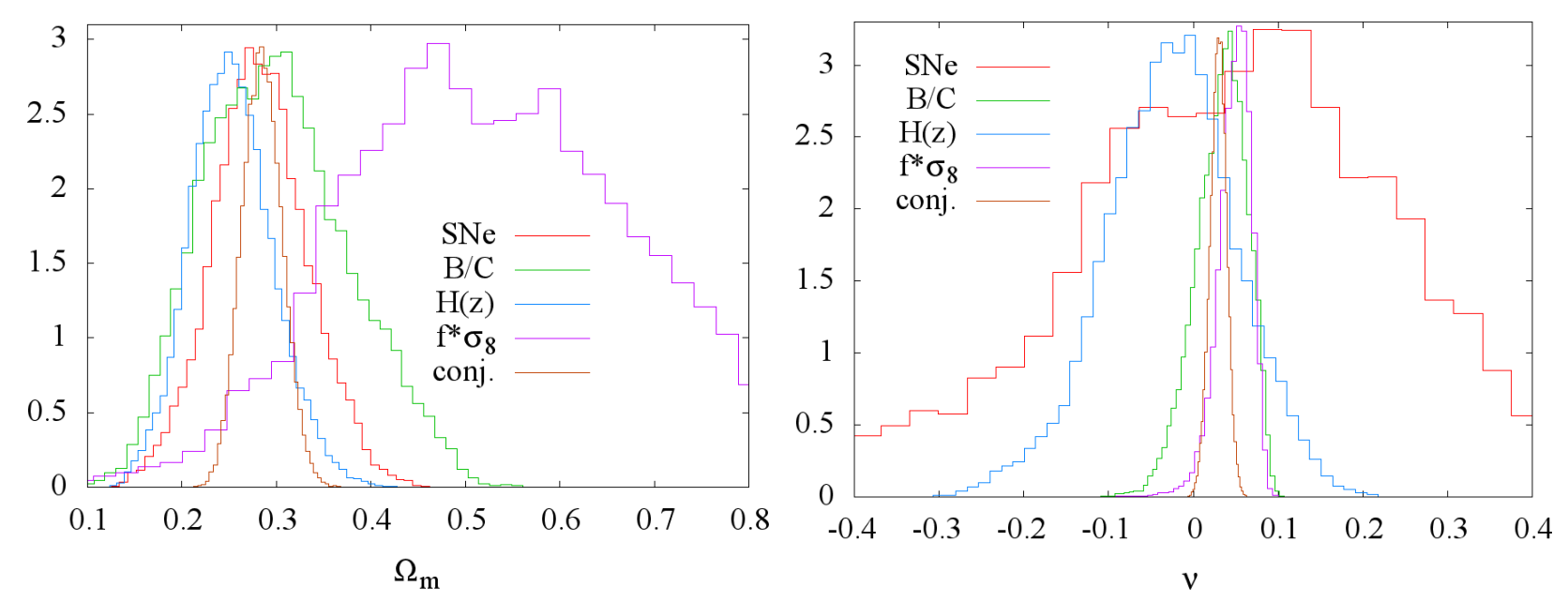

Figura 6.11: Distribuição dos parâmetros do modelo obtida das análises separadas e conjunta. Com o objetivo de comparar as distribuições, estas foram normalizadas individualmente, de forma a todas terem a mesma altura. Em particular nota-se que os dados de Supernovas apresentam um alto grão de degenerescência em relação ao parâmetro $v$, seguidos pelos dados de $H(z)$, enquanto que os dados de $\mathrm{BAO} / \mathrm{CMB}$ e da função de crescimento proporcionam uma restrição bem definida para $v>0$. Por outro lado, a degenerescência de $\Omega_{m}$ em relação aos dados da função de crescimento é muito grande na presencia de $v$, o que da obviamente uma grande restrição para este último parâmetro ao considerar a análise conjunta.

de matéria; e no caso particular de uma densidade inicial nula para os fluidos materiais, o período inflacionário não teria fim. Como consequência direta, para uma densidade inicial de matéria diferente de zero, o universo apresenta uma saída natural e inevitável do período inflacionário para o período de domínio da radiação, resolvendo o problema da saída da inflação.

Adicionalmente, devido à troca de energia entre a energia escura e a radiação, durante o período inflacionário a densidade de energia da radiação cresce conforme uma potência do fator de escala até atingir uma fração igual a $(1-v)(2 / n)(1+2 / n)^{-(1+2 / n)}$ da densidade critica inicial (no começo do período inflacionário) gerando um universo pós-inflacionário quente dominado por radiação.

Após o período inflacionário, a densidade de energia do fluido material dominante decresce como $a^{3(1+\omega)(1-v)}$, conforme a previsão do modelo $\Lambda \mathrm{CDM}$ corrigida pela dinâmica do vácuo devida ao termo $3 v H^{2}$, enquanto a densidade do vácuo decresce como $\rho_{\Lambda} \approx \rho v /(1-v)$ aliviando o problema da coincidência. Se $v \ll 1$ o presente modelo gera um universo recente tipo $\Lambda$ CDM mais correções da ordem de $v$. Inclusive agora, quando o universo estaria dominado pela densidade do vácuo gerando o período de expansão acelerada observado, a energia do vácuo apresentaria uma densidade constante mais uma pequena correção dinâmica da ordem de $v H^{2}$.

Este modelo é caracterizado principalmente por conduzir o universo de uma era primordial de Sitter para a era de Sitter final, na que estamos entrando atualmente, passando por um período desacelerado dominado pelos fluidos materiais.

Foi possível, fazendo um ajuste fino no parâmetro $n \approx 2 \times 10^{-2}$, extrair do modelo um espectro primordial (índice escalar avaliado escala pivô) que é compatível com os valores observados [Planck Collaboration, 2014]. Para isto se assumiu que as perturbações da radiação dominam sobre qualquer 

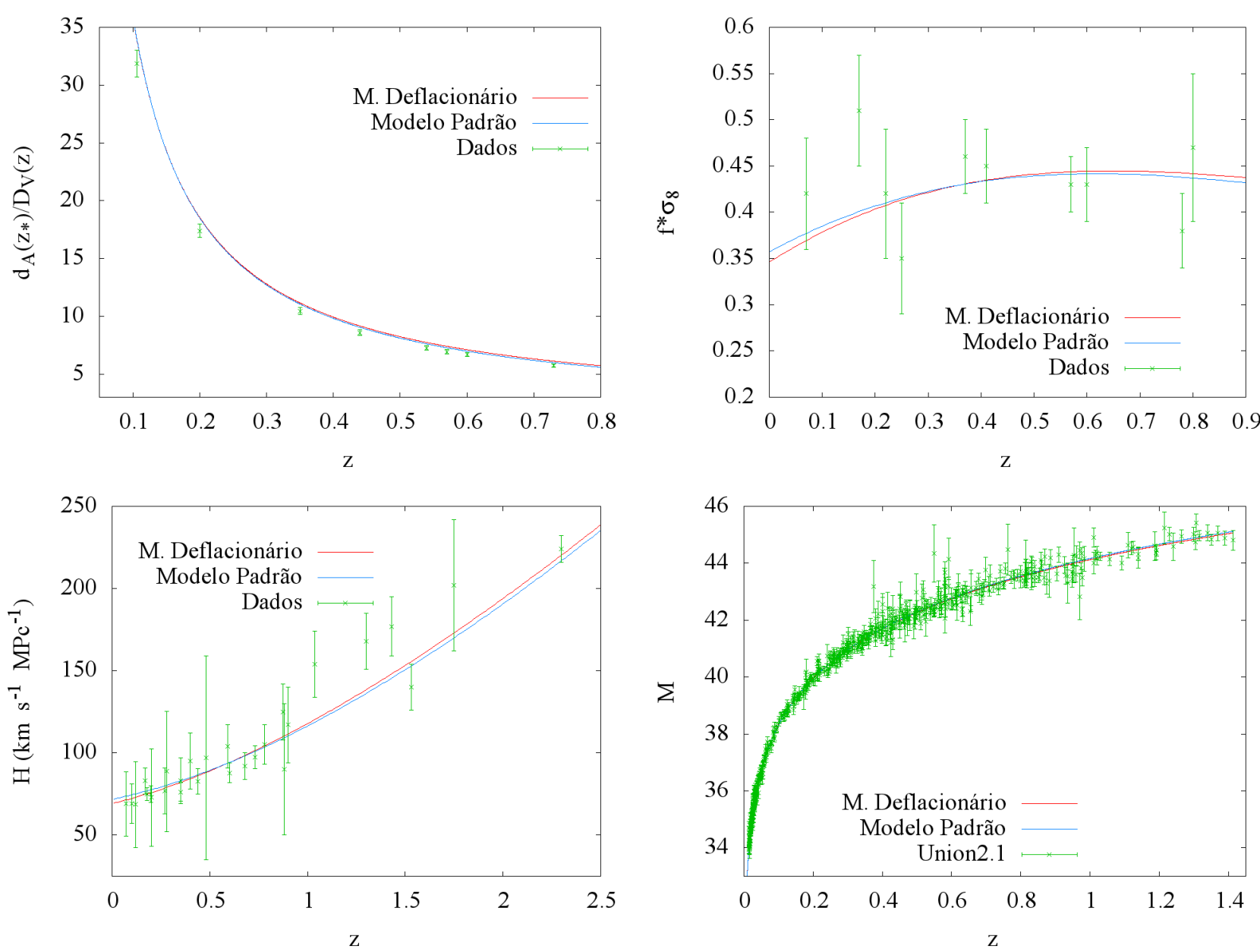

Figura 6.12: Melhor ajuste do modelo deflacionário e do modelo padrão.

Se mostra o melhor ajuste conjunto do presente modelo (parâmetros da tabela 1), e do modelo padrão $\left(v=0, \Omega_{m}=0.314, H_{0}=67.4 \mathrm{Km} \mathrm{Mpc}^{-1} s^{-1}\right.$ e $\sigma_{8}=0.834$ [Planck Collaboration, 2014]) com os dados observacionais.

perturbação da energia escura, mesmo quando a densidade de energia da radiação é subdominante durante a inflação. Referente ao ajuste do modelo às observações no universo recente, encontramos que o parâmetro livre $v$ deve ser da ordem de $v=0.029 \pm 0.01$, que é compatível com o zero (equivalente ao modelo padrão) somente no limite de $3 \sigma$.

Finalmente, vale a pena ressaltar que o presente modelo não prediz o valor numérico para a densidade do vácuo remanescente constante, mas prevê a sua existência e sugere que ele seja muito menor que a escala de energia típica do período inflacionário, devido a seu rápido decrescimento após o fim da inflação. 


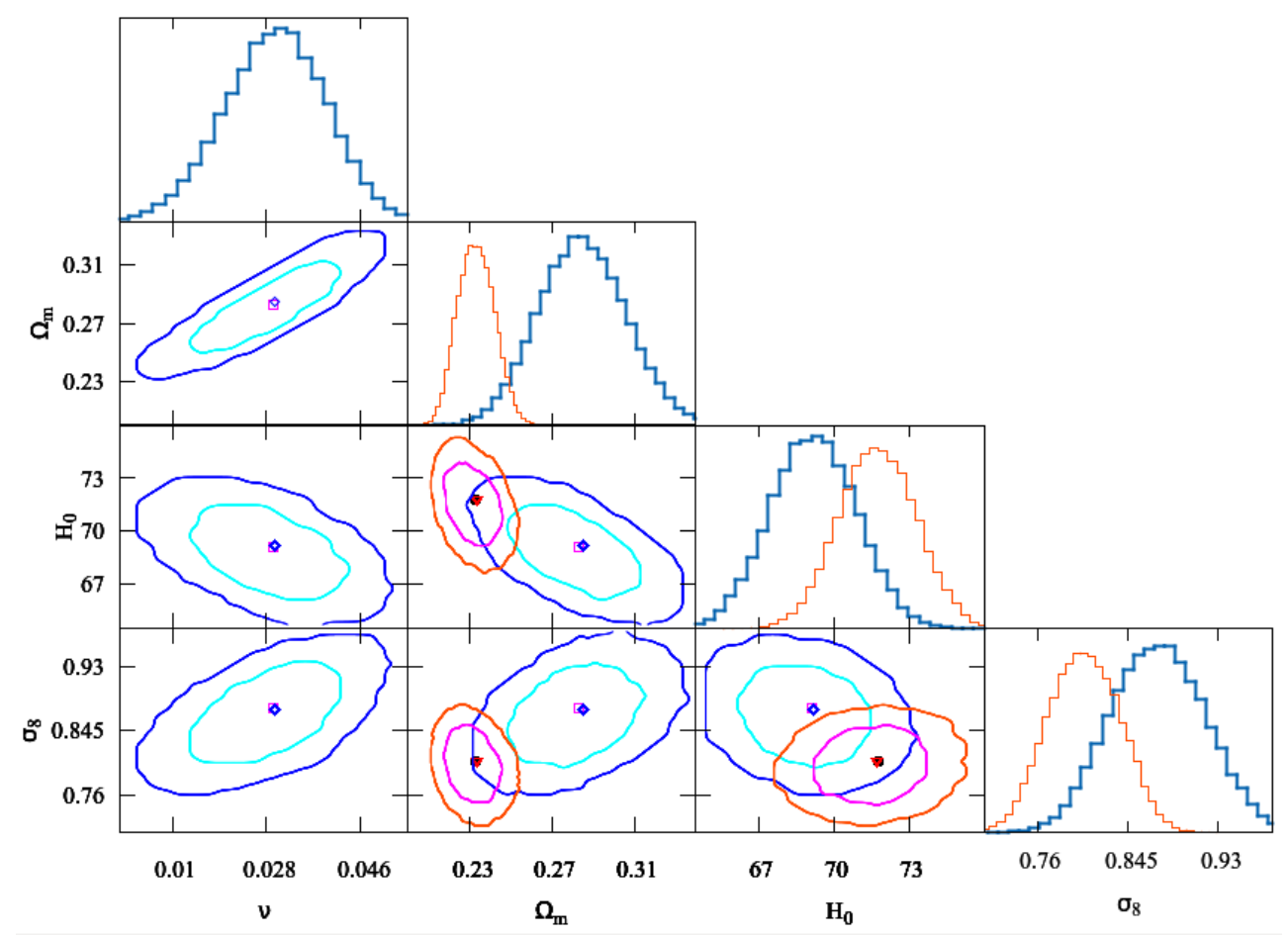

Figura 6.13: Distribuição de probabilidade dos parâmetros ajustados usando a análise conjunta dos dados de Supernova, BAO/CMB, H(z) e função de crescimento. Em cor azul o modelo deflacionário, em cor vermelha o modelo padrão.

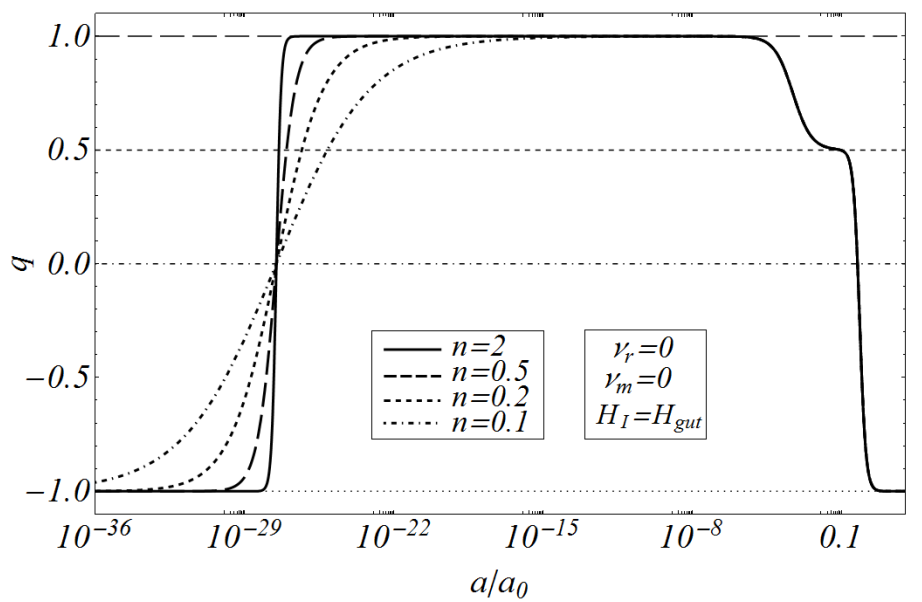

Figura 6.14: Parâmetro de desaceleração no modelo completo com $\Lambda=\Lambda_{c}+H^{2}\left(3 v+\left(H / H_{I}\right)^{n}\right)$. Juntando as soluções do parâmetro de Hubble em função do fator de escala para o universo primordial e para o universo recente podemos calcular o parâmetro de desaceleração $q=-a H^{\prime} / H-1$ ao longo de toda a historia cósmica mostrado na figura acima para alguns valores do parâmetro $n$. 



\section{MODELO DEFLACIONÁRIO E SEGUNDA LEI DA TERMODINÂMICA}

O objetivo do presente capítulo é mostrar que o modelo de universo, cuja cinemática foi estudada no capítulo 6, é permitido termodinamicamente. Especificamente vamos nos concentrar no estudo da entropia associada ao horizonte cosmológico aparente e a seu conteúdo energético, mostrando que é sempre uma função que cresce com o fator de escala e que sua segunda derivada temporal é negativa durante o período de domínio do vácuo remanescente, enquanto que é positiva (não pode ser um estado final para a evolução térmica do universo) durante o período primordial acelerado e durante os períodos de domínio dos fluidos materiais (usualmente radiação e matéria escura fria). Lembramos que no presente capítulo faremos uso das unidades físicas de $c, \hbar$ e $k_{B}$.

\subsection{Entropia associada ao horizonte cosmológico aparente}

Para métrica FRW plana

$$
d s^{2}=-d t^{2}+a^{2}\left(d r^{2}+r^{2} d \Omega^{2}\right)
$$

a condição de formação de um horizonte aparente $r_{h}$ (B.8) assume a forma simples

$$
\left.R_{232}^{3}\right|_{h}=\left.g^{\mu v} \mathscr{R}_{\mu} \mathscr{R}_{, v}\right|_{h}=r_{h}^{2} \mathcal{H}^{2}-1=0, \quad \mathscr{R}_{h}=H^{-1},
$$

onde $R_{232}^{3}$ é a componente 3232 do tensor de Riemann, e $\mathscr{R} \equiv a r$.

Dado que o escalar de Ricci é maior que cero para sistemas com geometria FRW e compostos por energia escura, matéria escura fria, bárions, neutrinos e radiação (ver equação (2.45)), teremos que o horizonte aparente é um horizonte atrapante [Faraoni, 2011] (segundo o formalismo de [Hayward, 1994]), e portanto sua temperatura associada é sempre positiva [Nielsen, 2009; Di Criscienzo et al., 2010; Vanzo et al., 2011; Iofa, 2015]. Com isto podemos associar naturalmente a dito horizonte a entropia (B.10) (ver também [Mimoso and Pavón, 2013]):

$$
\mathcal{S}_{h}=\frac{k_{B}}{4 \ell_{p l}^{2}} \mathscr{A}_{h},
$$

onde $\mathscr{A}_{h}=4 \pi \mathscr{R}_{h}^{2}=4 \pi H^{-2}$ é a área do horizonte, e $\ell_{p l}$ é o comprimento de Planck.

Aplicando a regra da cadeia geral

$$
\frac{d x}{d t}=\frac{d a}{d t} \frac{d x}{d a} \equiv a H x^{\prime}, \quad \frac{d^{2} x}{d t^{2}}=\frac{d^{2} a}{d t^{2}} x^{\prime}+\left(\frac{d a}{d t}\right)^{2} x^{\prime \prime},
$$


à expressão para a entropia (7.3), associada ao horizonte aparente do universo plano, temos que sua primeira e segunda derivadas temporais são

$$
\frac{d \mathcal{S}_{h}}{d t}=-\frac{2 \pi k_{B}}{\ell_{p l}^{2}} a \frac{H^{\prime}}{H^{2}}>0, \quad \frac{d^{2} \mathcal{S}_{h}}{d t^{2}}=-\frac{2 \pi k_{B}}{\ell_{p l}^{2}} a \frac{H^{\prime}}{H}\left[1-2 a \frac{H^{\prime}}{H}+\frac{H^{\prime \prime}}{H^{\prime}}\right] .
$$

Nota-se que a primeira derivada é sempre maior que zero assumindo a condição fraca de energia, já que das equações de Einstein podemos obter a expressão:

$$
-H^{\prime}=4 \pi G\left(\rho_{T}+\mathcal{P}_{T}\right)>0
$$

onde $\rho_{T}$ e $\mathcal{P}_{T}$ são a densidade de energia total e a pressão total do conteúdo energético do universo.

\subsubsection{Universo primitivo}

Se usarmos a expressão (7.3) para a entropia associada ao horizonte aparente do universo, e o parâmetro de Hubble dado por (6.7) obtemos que

$$
\mathcal{S}_{h}=\frac{k_{B} \mathscr{A}_{h}}{4 \ell_{p l}^{2}}=\frac{\pi k_{B} c^{2}}{\ell_{p l}^{2} H^{2}}=\frac{\pi k_{B} c^{2}}{\ell_{p l}^{2} H_{I}^{2}}(1+\chi)^{2 / n},
$$

onde definimos $\chi=\chi(a) \equiv\left(a / a_{\star}\right)^{n \xi} /(\xi-1), \operatorname{com} \xi \equiv 3(1+\omega)(1-v) / 2$. Obtemos assim que a sua primeira derivada temporal pode ser escrita como:

$$
\frac{d \mathcal{S}_{h}}{d t}=\frac{2 \pi k_{B} c^{2}}{H_{I} \ell_{p l}^{2}} \xi \chi(1+\chi)^{-1+1 / n}
$$

é evidentemente maior que zero para $\xi>0(\xi>1$ equivale a que $v<(1+3 \omega) /(3(1+\omega))$, o que é necessário para que o universo seja dominado pelo fluido material após o período inflacionário). A segunda derivada pode ser escrita como:

$$
\frac{d^{2} \mathcal{S}_{h}}{d t^{2}}=\frac{2 \pi k_{B} c^{2}}{\ell_{p l}^{2}} \xi^{2} \frac{\chi(n+\chi)}{(1+\chi)^{2}}
$$

é sempre maior que zero.

\subsubsection{Universo recente}

No caso de um universo composto por um fluido material e uma densidade do vácuo dinâmica, a expressão para a entropia do horizonte aparente (7.3) é

$$
\mathcal{S}_{h}=\frac{k_{B} \pi}{\ell_{p l}^{2}} \frac{c^{2}}{H^{2}}=\frac{\pi k_{B} c^{2}}{\ell_{p l}^{2} H_{0}^{2}} \frac{1-v}{\Omega_{m}^{0} y+\Omega_{\Lambda}^{0}-v}
$$


onde usamos a expressão (6.51) para o parâmetro de Hubble, e $y \equiv\left(a / a_{0}\right)^{-2 \xi}$. Se agora usamos a expressão (7.5) para a primeira derivada da entropia do horizonte obtemos

$$
\frac{d \mathcal{S}_{h}}{d t}=\frac{2 \pi k_{B} c^{2}}{H_{0} \ell_{p l}^{2}} \frac{\xi \Omega_{\omega} y \sqrt{1-v}}{\left(\Omega_{\Lambda}-v+\Omega_{\omega} y\right)^{3 / 2}}
$$

que é maior que zero para $\xi>0$, enquanto que a segunda derivada temporal pode ser escrita como:

$$
\frac{d^{2} \mathcal{S}_{h}}{d t^{2}}=\frac{2 \pi k_{B} c^{2}}{\ell_{p l}^{2}} \xi^{2} \Omega_{\omega} y \frac{\Omega_{\omega} y-2\left(\Omega_{\Lambda}-v\right)}{\left(\Omega_{\Lambda}-v+\Omega_{\omega} y\right)^{2}} .
$$

Nota-se na expressão acima que a segunda derivada temporal da entropia do horizonte é maior que zero para $a \ll a_{0}(y \gg 1)$ sempre que $\xi>0$, enquanto é menor que zero para $a \gg a_{0}(y \ll 1)$.

\subsection{TEMPERATURA DE UM FLUIDO IDEAL}

Considerando a expansão do universo como sendo um processo que acontece quase-estaticamente em regiões menores que o horizonte aparente, podemos usar as equações da termodinâmica de fluidos para modelar a evolução da temperatura e da entropia do conteúdo material. Em geral, considerando individualmente a evolução termodinâmica de cada fluido (parametrizando a sua interação no potencial químico), esta deve ser descrita pelas equações:

$$
\mathrm{T} \mathcal{S}=\mathcal{P} V+U-\mu N, \quad \mathcal{S} d \mathrm{~T}=V d \mathcal{P}+N d \mu
$$

onde $U=c^{2} \rho V, \mathcal{S}$ e $N$ são a energia interna, a entropia e o número de partículas para um fluido com temperatura $T$, pressão $\mathcal{P}$ e potencial químico $\mu$ contido em um volume arbitrário $V$. Se o fluido possui a equação de estado $\mathcal{P}=\omega \rho$ as equações acima posem ser reescritas como:

$$
\mathrm{T} \mathcal{S}=(1+\omega) c^{2} \rho V-\mu N, \quad \mathcal{S} d \mathrm{~T}=\omega c^{2} V d \rho+N d \mu .
$$

Agora, a maior contribuição para a entropia dentro do horizonte aparente do universo vai estar dada pelo fluido material dominante (radiação ou matéria escura, a saber). Por outro lado, o vácuo $\Lambda$ (definido como $\omega_{\Lambda}=-1$ e $\mu_{\Lambda}=0$ ) apresenta uma entropia constante.

No caso da radiação, e para um potencial químico nulo (assumindo que o vácuo somente transfere energia cinética para os fótons, mas não cria novos fótons) as equações (7.14) se reduzem ao resultado usual

$$
4 d \ln \left(\mathrm{T}_{\gamma}\right)=d \ln \left(\rho_{\gamma}\right), \quad \rightarrow \quad \frac{\rho_{\gamma}}{\rho_{\gamma}^{0}}=\left(\frac{\mathrm{T}_{\gamma}}{\mathrm{T}_{\gamma}^{0}}\right)^{4} .
$$

No caso geral de um fluido com equação de estado $\mathcal{P}=\omega \rho(\operatorname{com} \omega \neq 0)$ e potencial químico nulo as expressões (7.14) resultam na relação [Lima and Maia, 1995; Lima and Santos, 1995; Lima, 1996; Silva et al., 2002, 2007; Lima and Pereira, 2008; Pereira and Lima, 2008]:

$$
(1+\omega) d \ln (\mathrm{T})=\omega d \ln (\rho), \quad \rightarrow \quad\left(\frac{\mathrm{T}}{\mathrm{T}^{0}}\right)^{1+\omega}=\left(\frac{\rho}{\rho^{0}}\right)^{\omega}
$$




\subsection{ENTROPIA DOS Fluidos CONTIDOS NO HORIZONTE}

Na presente descrição, onde a energia escura é modelada como sendo a densidade do vácuo (que não carrega entropia), a contribuição principal para a entropia de uma região com volume igual ao volume do horizonte aparente do universo é dada pela entropia do fluido material dominante contida no horizonte, que no caso do universo primitivo corresponde à radiação, enquanto que para o universo recente corresponde à matéria escura fria.

\subsubsection{Instabilidade do período inflacionário}

A entropia associada à radiação contida dentro de um volume esférico com raio igual ao horizonte aparente do universo pode ser calculada a partir da relação termodinâmica:

$$
\mathrm{T} d \mathcal{S}=d U+\mathcal{P} d \mathscr{V}=\frac{4 \pi c^{2}}{3} d\left(\rho \mathscr{R}_{h}^{3}\right)+\frac{4 \pi c^{2}}{3} \frac{\rho}{3} d\left(\mathscr{R}_{h}^{3}\right),
$$

onde $\mathscr{R}_{h} \equiv(c / H)$ (é o raio do horizonte aparente para um universo plano, sendo solução à equação (B.8) usando a métrica FRW plana), também consideramos o volume da região dentro do horizonte aparente como sendo $\mathscr{V}=4 \pi \mathscr{R}_{h}^{3} / 3$, e a energia total contida neste volume como $U=c^{2} \rho \mathscr{V}$.

Se usarmos a expressão (7.16) para a temperatura do fluido material obtemos que a entropia da radiação dentro do horizonte aparente durante os estágios primordiais do universo é:

$$
\mathcal{S}_{r}=\left(\frac{4}{3}\right)^{2} \pi c^{5} \frac{\rho_{\star}^{1 / 4}}{\mathrm{~T}_{\star}} \frac{\rho^{3 / 4}}{H^{3}}
$$

onde $T_{\star}$ e $\rho_{\star}$ são a temperatura e a densidade de energia da radiação quando $a=a_{\star}$ (ou seja, quando $\left.d^{2} a / d t^{2}=0\right)$. Com isso, a sua primeira derivada temporal é

$$
\frac{d \mathcal{S}_{r}}{d t}=\mathcal{S}_{r} \frac{4 H_{I} \xi}{3}(2 \chi+n)(1+\chi)^{-1-1 / n}
$$

é maior que zero para $\xi>0$, enquanto a segunda derivada pode ser escrita como

$$
\frac{d^{2} \mathcal{S}_{r}}{d t^{2}}=\mathcal{S}_{r} \frac{3 H_{I}^{2} \xi^{2}}{16}(1+\chi)^{-2-2 / n}\left(4 \chi^{2}+4 n \chi(4-n)+3 n^{2}\right),
$$

é sempre maior que zero para $n<4$, mas para todo $n$ é maior que zero tanto para $a \ll a_{\star}(\chi \ll 1)$ quanto para $a \gg a_{\star}(\chi \gg 1)$.

Assim, durante o período primordial de expansão, a entropia total $\left(\mathcal{S}_{T}=\mathcal{S}_{h}+\mathcal{S}_{r}\right)$ (entropia associada ao horizonte mais a entropia associada ao conteúdo material dele) é sempre uma função crescente $\left(\partial_{t} \mathcal{S}_{T}>0\right.$ já que tanto $\partial_{t} \mathcal{S}_{h}>0$ como $\left.\partial_{t} \mathcal{S}_{r}>0\right)$ e convexa $\left(\partial_{t}^{2} \mathcal{S}_{T}>0\right.$ já que $\partial_{t}^{2} \mathcal{S}_{h}>0$ como também $\left.\partial_{t}^{2} \mathcal{S}_{r}>0\right)$ para $n<4$ e $\xi>1$, portanto o dito período é instável termodinamicamente como predito por [Mimoso and Pavón, 2013]. 


\subsubsection{Estabilidade da era de Sitter final do universo}

A entropia associada ao conteúdo material do horizonte aparente (neste caso poeira) pode ser calculada como

$$
\mathcal{S}=\frac{U+\mathcal{P} \mathscr{V} /}{\mathrm{T}}=\frac{U}{\mathrm{~T}}=\frac{c^{2} \rho_{m} \mathscr{V} / h}{\mathrm{~T}}=\frac{4 \pi c^{3}}{3 \mathrm{~T}} \frac{\rho}{H^{3}}
$$

onde $\mathrm{T}=$ cte é a temperatura associada a um fluido com pressão zero, ver [Lima and Maia, 1995; Lima and Santos, 1995; Lima, 1996; Silva et al., 2002; Lima and Pereira, 2008; Pereira and Lima, 2008], e deve ser da ordem da massa do campo de matéria escura em unidades naturais. Usando a expressão (6.57) para o parâmetro de Hubble e (6.58) para a densidade de energia da matéria obtemos:

$$
\frac{d \mathcal{S}_{c}}{d t}=\frac{\hbar c^{2}}{2 \ell_{p l}^{2} \top}(1-v) \xi y \frac{\Omega_{\omega} y-2\left(\Omega_{\Lambda}-v\right)}{\left(\Omega_{\Lambda}-v+\Omega_{\omega} y\right)^{2}}\left\{\begin{array}{cc}
>0, & a \ll a_{0}, \\
<0, & a \gg a_{0} .
\end{array},\right.
$$

$\mathrm{e}$

$$
\frac{d^{2} \mathcal{S}_{c}}{d t^{2}}=-\frac{2 \hbar c^{2} H_{0}}{\ell_{p l}^{2} \top} \xi^{2}\left(\Omega_{\Lambda}-v\right) \sqrt{1-v} y \frac{2 \Omega_{\omega} y-\left(\Omega_{\Lambda}-v\right)}{\left(\Omega_{\Lambda}-v+\Omega_{\omega} y\right)^{5 / 2}}\left\{\begin{array}{cc}
<0, & a \ll a_{0}, \\
>0, & a \gg a_{0} .
\end{array},\right.
$$

Este resultado não é um problema, já que:

$$
\lim _{a \ll a_{0}}\left|\frac{d^{2} \mathcal{S}_{c} / d t^{2}}{d^{2} \mathcal{S}_{h} / d t^{2}}\right|=\frac{2 \sqrt{1-v}\left(\Omega_{\Lambda}-v\right)}{\pi \Omega_{\omega}^{3 / 2}} \frac{\hbar H_{0}}{k_{B} \mathrm{~T}} y^{-1 / 2} \sim y^{-1 / 2} \mathcal{O}\left(\mathrm{T}_{0} / \mathrm{T}\right) \lll 1
$$

e

$$
\lim _{a \gg a_{0}}\left|\frac{d \mathcal{S}_{c} / d t}{d \mathcal{S}_{h} / d t}\right|=\lim _{a \gg a_{0}}\left|\frac{d^{2} \mathcal{S}_{c} / d t^{2}}{d^{2} \mathcal{S}_{h} / d t^{2}}\right|=\frac{\sqrt{1-v} \sqrt{\Omega_{\Lambda}-v}}{2 \pi \Omega_{\omega}} \frac{\hbar H_{0}}{k_{B} \mathrm{~T}} \sim \mathcal{O}\left(\mathrm{T}_{0} / \mathrm{T}\right) \ll 1
$$

onde $\mathrm{T}_{0}$ é da ordem da escala de energia do universo hoje, enquanto $\mathrm{T}$ é da ordem da escala de energia do universo quando a matéria escura torna-se não relativista.

Com isso, durante o período de domínio da matéria escura fria obtemos que a entropia total $\mathcal{S}_{\mathcal{T}}=$ $\mathcal{S}_{h}+\mathcal{S}_{c}$ é uma função crescente $\left(\partial_{t} \mathcal{S}_{T}>0\right.$ já que $\partial_{t} \mathcal{S}_{h}>0$ também como $\left.\partial_{t} \mathcal{S}_{c}>0\right)$ e convexa $\left(\partial_{t}^{2} \mathcal{S}_{T} \approx\right.$ $\left.\partial_{t}^{2} \mathcal{S}_{h}>0\right)$, não levando o universo para um estado estável desde o ponto de vista termodinâmico.

Por outro lado, durante o período de domínio do vácuo remanescente (para $a \gg a_{0}$ que é equivalente a $y \ll 1$ sempre que $\xi>0$ ) obtemos que a entropia total é uma função crescente $\partial_{t} \mathcal{S}_{T} \approx \partial_{t} \mathcal{S}_{h}>0$ e convexa $\partial_{t}^{2} \mathcal{S}_{T} \sim \partial_{t}^{2} \mathcal{S}_{h}<0$, levando o universo a um estágio de estabilidade termodinâmica, já que a entropia do horizonte prevalece sobre a entropia da matéria contida nele. 



\section{UNIVERSO DEFLACIONÁRIO E CURVATURA ESPACIAL}

Como já vimos, no contexto de um universo FRW plano, uma densidade do vácuo dinâmica pode gerar uma era de Sitter nos primórdios do universo (como também gerar a aceleração recente) reduzindo o setor inflacionário, e aliviando o problema da constante cosmológica. O tipo de modelo estudado anteriormente (com uma densidade do vácuo que decresce monotonicamente enquanto o universo se expande) não possui o problema da saía do período primordial acelerado para o período de domínio da radiação, e não precisa de um mecanismo adicional para reaquecer o universo. O objeto de este capítulo é mostrar que independentemente do sinal da curvatura espacial do universo FRW, o universo apresenta um estágio de Sitter primordial, mas é um estágio temporário no caso em que a curvatura espacial é não nula [Lima et al., 2015b].

\subsection{O MODELO}

Para um universo descrito pela métrica de FRW (2.17)

$$
d s^{2}=d t^{2}-a(t)^{2}\left(\frac{1}{1-\kappa r^{2}} d r^{2}+r^{2} d \Omega^{2}\right)
$$

e composto por uma densidade do vácuo que interage com um fluido ideal com equação de estado:

$$
\mathcal{P}=(\gamma-1) \rho,
$$

onde $\mathcal{P}$ e $\rho$ são a pressão e a densidade de energia do fluido, enquanto que $\gamma \in[1,2]$, as equações de Friedmann tomam a forma:

$$
8 \pi G \rho_{T} \equiv 8 \pi G \rho+\Lambda=3\left(H^{2}+\frac{\kappa}{a^{2}}\right)
$$

e

$$
8 \pi G \mathcal{P}_{T} \equiv 8 \pi G \mathcal{P}-\Lambda=-\frac{2}{a} \frac{d^{2} a}{d t^{2}}-\left(H^{2}+\frac{\kappa}{a^{2}}\right),
$$

onde $\mathcal{P}_{T}$ e $\rho_{T}$ são a pressão de densidade de energia total do sistema.

A existência de duas componentes de energia no sistema permite introduzir a seguinte razão:

$$
\beta=\frac{\rho_{v}-\rho_{v 0}}{\rho_{T}},
$$


onde $\rho_{v 0}$ é um termo constante na densidade do vácuo mais geral. O parâmetro $\beta$ quantifica a variação de $\Lambda(t)$ e pode gerar diferentes cosmologias dependendo das suas características:

1. Se $\rho_{v}=\rho_{v 0}$, então $\beta=0$, dando como resultado o modelo padrão $\Lambda$ CDM:

$$
\Lambda=\Lambda_{0}
$$

2. Para $\rho_{v 0} \ll \rho_{v}$, a razão define a fração de vácuo em relação à densidade total. Se esta razão é constante ao longo de alguma parte da história cósmica se obtém [Carvalho et al., 1992]:

$$
\Lambda(t)=3 \beta\left(H^{2}+\frac{\kappa}{a^{2}}\right)
$$

3. Se a razão $\beta$ é constante ao longo de toda a história cósmica (após a inflação) obtemos que:

$$
\Lambda(t)=\Lambda_{\text {const }}+3 \beta\left(H^{2}+\frac{\kappa}{a^{2}}\right)
$$

onde o caso $\kappa=0$ tem sido sugerido a partir das propriedades gerais do grupo de renormalização em ambientes cosmológicos [Shapiro and Solà, 2002].

4. No caso mais geral, a razão $\beta$ pode não ser uma constante, ou seja $\beta=\beta(t)$, o que permite expressar a densidade do vácuo como sendo:

$$
\rho_{v}=\rho_{v 0}+\beta(t) \rho_{T}
$$

ou

$$
\Lambda(t)=\Lambda_{\text {const }}+3 \beta(t)\left(H^{2}+\frac{\kappa}{a^{2}}\right)
$$

Se expandirmos o parâmetro $\beta$ como:

$$
\beta(t)=v+(1-v)\left(\frac{H}{H_{I}}\right)^{n},
$$

onde o pre-fator $(1-v)$ de $H_{I}^{-n}$ foi introduzido por conveniência matemática, e o parâmetro $H_{I}$ representa a função de Hubble característica do período inflacionário. Este ansatz generaliza o modelo da referências [Freese et al., 1987; Lima and Trodden, 1996] e [Lima et al., 2013], usando tanto um fator com uma potência arbitrária de $H$, como um termo de curvatura $\kappa$.

Por simplicidade vamos usar o ansatz (8.8) para a densidade do vácuo $\Lambda(t) \operatorname{com} v=0$, com o que obtemos:

$$
\Lambda=\Lambda_{\infty}+3\left(\frac{H}{H_{I}}\right)^{n}\left(H^{2}+\frac{\kappa}{a^{2}}\right)
$$

enquanto que as equações de Friedmann podem ser reescritas da forma:

$$
\begin{gathered}
\frac{d H}{d t}=-4 \pi G \gamma \rho+\frac{\kappa}{a^{2}}, \\
8 \pi G \rho=3\left[1-\left(\frac{H}{H_{I}}\right)^{n}\right]\left(H^{2}+\frac{\kappa}{a^{2}}\right)-\Lambda_{\infty} .
\end{gathered}
$$


Para $H \lesssim H_{I}$ (e dado que $\left.\Lambda_{\infty} \lll H_{I}^{2}\right) \Lambda \approx 3\left(H^{2}+\kappa / a^{2}\right)$ enquanto que a densidade de energia do fluido material permanece desprezível levando o universo para um período inflacionário dominado pelo vácuo. Após o período inflacionário $H \ll H_{I}$ mas ainda quando $H^{2} \gg \Lambda_{\infty}$, a densidade do vácuo torna-se desprezível. Das equações (8.9) e (8.11) temos que a transição entre o período inflacionário dominado pelo vácuo, e o posterior período dominado pelo fluido material ocorre quando

$$
\left(\frac{H_{\star}}{H_{I}}\right)^{n}=\frac{1}{2} .
$$

Para esta época, $\left(t_{\star}\right)$, a densidade de energia material é da ordem da densidade crítica do período inflacionário:

$$
8 \pi G \rho_{\star}=3\left(H_{\star}^{2}+\frac{\kappa}{a_{\star}^{2}}\right) \lesssim \mathcal{O}\left(\Lambda_{I}\right),
$$

descrevendo um universo quente, dominado pelo fluido material, capaz de gerar o processo de nucleossínteses, independentemente do sinal de $\kappa$.

\subsection{CASOS LIMITE}

Combinando as equações (8.7) e (8.8) com as equações de Friedmann se obtém a seguinte equação diferencial que descreve a evolução do fator de escala:

$$
a \frac{d^{2} a}{d t^{2}}+\frac{3 \tilde{\gamma}-2}{2}\left[\left(\frac{d a}{d t}\right)^{2}+\kappa\right]-\frac{\gamma}{2} \Lambda_{\infty} a^{2}=0
$$

onde

$$
\tilde{\gamma}=\tilde{\gamma}(H) \equiv \gamma\left[1-\left(\frac{H}{H_{I}}\right)^{n}\right]
$$

Este parâmetro permite fazer a separação entre os cenários com $H \approx H_{I}$ (estágio inflacionário), e $H \ll H_{I}$ mas $H^{2} \gg \Lambda_{\infty}$ (estágio intermediário) da seguinte forma:

1. Durante o universo primitivo, quando $H \approx H_{I}$ e $\Lambda_{\infty}$ é desprezível, o parâmetro $\beta$ se torna

$$
\beta\left(H \approx H_{I}\right) \approx 1
$$

devido a que $\tilde{\gamma}\left(H=H_{I}\right)=0$, e portanto a equação (8.12) assume a forma

$$
a \frac{d^{2} a}{d t^{2}}-\left[\left(\frac{d a}{d t}\right)^{2}+\kappa\right]=0
$$

que possui a seguinte solução

$$
a(t)= \begin{cases}\cosh \left(H_{I} t+c_{\star}\right), & \kappa>0 \\ a_{\star} \exp \left(H_{I} t\right), & \kappa=0 \\ \sinh \left(H_{I} t+c_{\star}\right), & \kappa<0\end{cases}
$$


onde $c_{\star}$ e $a_{\star}$ são constantes de integração, enquanto que $H_{I}^{2}(\kappa \neq 0)=|\kappa|$. Para $H^{2} \gg \Lambda_{\infty}$ as densidades do vácuo de do fluido material podem ser escritas como:

$$
\begin{aligned}
& \Lambda=3\left(\frac{H}{H_{I}}\right)^{n}\left(H^{2}+\frac{\kappa}{a^{2}}\right) \stackrel{H \rightarrow H_{I}}{\longrightarrow} 3\left(H^{2}+\frac{\kappa}{a^{2}}\right), \\
& 8 \pi G \rho=3\left[1-\left(\frac{H}{H_{I}}\right)^{n}\right]\left(H^{2}+\frac{\kappa}{a^{2}}\right) \stackrel{H \rightarrow H_{I}}{\longrightarrow} 0 .
\end{aligned}
$$

Esta solução para $\rho$, junto com à equação (8.10), mostram que para $\kappa \neq 0$ a solução (8.15) com $H \sim H_{I}$ é transitória, já que $d H /\left.d t\right|_{H \sim H_{I}} \approx \kappa / a^{2}$. Assim, para $\kappa>0$ o universo começa com um período acelerado dominado pelo termo de curvatura em $\Lambda^{1}$, seguido de um domínio por parte do termo $H^{n}$ de $\Lambda$ (descrita pela solução (8.15)), mas ainda acelerado. No caso em que $\kappa<0$ é assumindo a condição fraca de energia, $\rho>0$, temos que o universo começa já no período de domínio do termo $H^{n}$, devido a que um eventual domínio do termo de curvatura esta ligado a $d H / d t<0$ e $H>H_{I}$, o que geraria um $\rho<0$. Por fim, para $\kappa=0$ o universo começa com uma era assimptoticamente de Sitter, dominada pelo termo em $H^{n}$ e gerando uma inflação exponencial.

2. Durante os estágios avançados do universo, quando $H \ll H_{I}$, a equação dinâmica (8.12) toma a forma:

$$
a \ddot{a}+\Delta\left(\dot{a}^{2}+\kappa\right)-(\Delta+1) \frac{\Lambda_{\infty}}{3} a^{2}=0, \quad \operatorname{com} \quad \Delta \equiv \frac{3 \gamma-2}{2} .
$$

A primeira integral de esta equação produz:

$$
H^{2}=a^{-3 \gamma}\left[H_{0}^{2}+\kappa-\frac{\Lambda_{\infty}}{3}\right]-\left[\frac{\kappa}{a^{2}}-\frac{\Lambda_{\infty}}{3}\right]
$$

E no limite de $a \gg 1$ as densidades de energia são:

$$
\begin{gathered}
\Lambda=\Lambda_{\infty}, \\
8 \pi G \rho=\frac{3}{a^{3 \gamma}}\left[H_{0}^{2}+\kappa-\frac{\Lambda_{\infty}}{3}\right],
\end{gathered}
$$

o que corresponde ao modelo $\Lambda C D M$.

\subsection{DINÂMICA DO MODELO}

A dinâmica do sistema esta determinada pelas equações (8.9), (8.10) e (8.11). Combinando as equações (8.10) e (8.11) para um universo primitivo, onde $\Lambda_{\infty}$ é desprezível, composto por vácuo e radiação, $\gamma=4 / 3$, e usando $v=0$ por simplicidade, obtemos:

$$
a E E^{\prime}+E^{2}+\left(1-2 E^{n}\right)\left[E^{2}-\Omega_{\kappa}^{\star}\left(\frac{a_{\star}}{a}\right)^{2}\right]=0
$$

\footnotetext{
${ }^{1}$ Durante o estágio dominado pelo termo de curvatura, a densidade total do sistema é proporcional a $a^{-2}$, para $\kappa>0$, e dado que a escala de Planck estabelece um limite de validade das teorias cassicas, o modelo não pode ser estendido até $a=0$, mas pode começar com $a \lesssim a_{\kappa}<a_{i}$, onde $a_{i}$ é definido como o limite superior do fator de escala no começo da inflação tal que esta resolve o problema do horizonte.
} 
onde a linha denota derivada em relação ao fator de escala, enquanto que

$$
E \equiv \frac{H}{H_{I}}, \quad \text { e } \quad \Omega_{\kappa}^{\star} \equiv \Omega_{\kappa}^{0}\left(\frac{a_{0} H_{0}}{a_{\star} H_{I}}\right)^{2} \sim 10^{-65}
$$

A equação (8.20) mostra que o termo de curvatura domina a dinâmica do sistema para $\left(a_{\star} / a\right)^{2} \lesssim 10^{-65}$, ou seja, para $a \lesssim a_{k} \equiv a_{\star} \times 10^{-32}$. Definimos $a_{i} \equiv a_{\star} \times 10^{-28}$ como sendo o valor máximo do fator de escala inicial do período inflacionário tal que um modelo de universo com idade finita consegue resolver o problema do horizonte.

As figuras 8.1 e 8.2 mostram a evolução do parâmetro de Hubble, das densidades de energia e do parâmetro de desaceleração em função do fator de escala. A figura 8.1 mostra o intervalo do fator de escala onde o modelo é bem descrito pela solução de Sitter, isto é para $a_{i}<a<a_{\star}$, onde $H \sim H_{I}$.
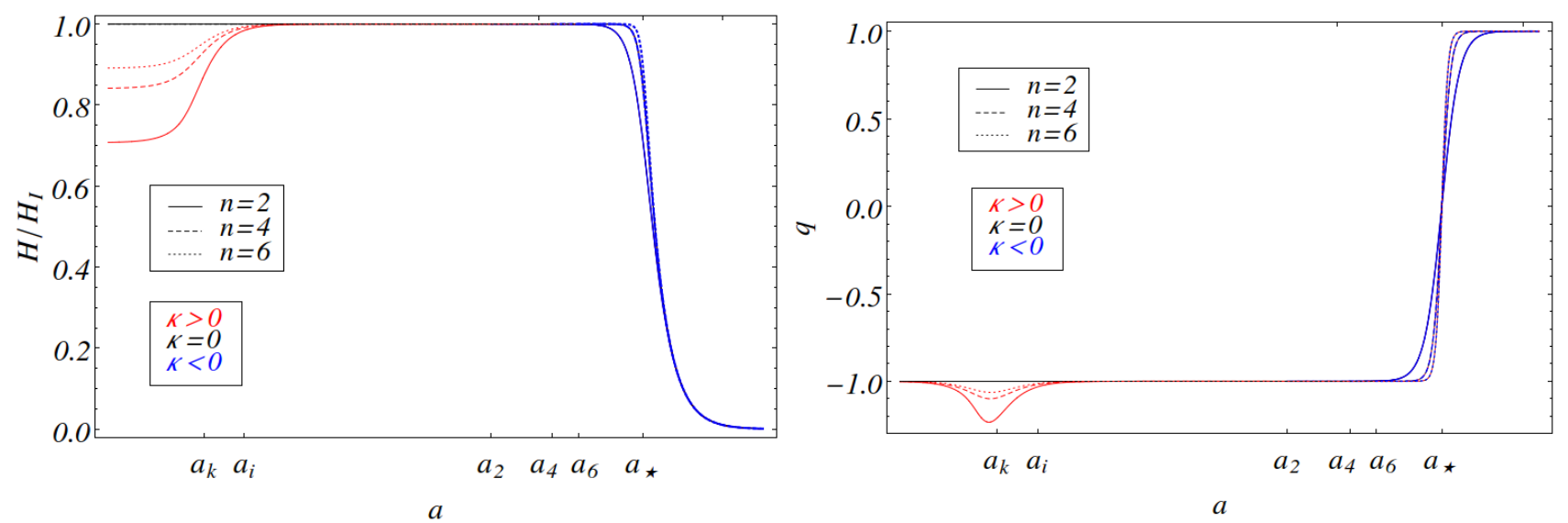

Figura 8.1: Dinâmica do modelo deflacionário com curvatura espacial.

Para $a>a_{\kappa}$ os efeitos da curvatura são desprezíveis, em particular, para $a \in\left(a_{\kappa}, a_{\star}\right)$ a dinâmica do sistema é bem descrita pela solução de Sitter. Já o parâmetro $n$ controla a rapidez com que ocorre a transição entre o período acelerado e o período dominado pela radiação. $a_{\star}$ representa o fim do período acelerado, enquanto que $\left(a_{i}, a_{\star}\right)$ representa o intervalo mínimo de dito período que consegue evitar o problema do horizonte. No caso em que $\kappa<0$ o modelo deflacionário é valido somente a partir de $a>a_{n}$, definido como $\rho\left(a_{n}\right)=0$. (Panel Esquerdo): Evolução do parâmetro de Hubble. Para $\kappa=0$ se tem que $H \rightarrow H_{I}$ quando $a \rightarrow 0$, enquanto que para $\kappa>0$ se tem que $H \rightarrow H_{\star}$. (Panel Direito): Evolução do parâmetro de desaceleração. Para $\kappa>0$ o parâmetro de desaceleração possui um pequeno desvio do valor $q=-1$, mas novamente converge para $q=-1$ quando $a \ll a_{\kappa}$.

Diferente da independência dos parâmetros de Hubble e de desaceleração, que são muito pouco afetados pelo sinal da curvatura para $a>a_{\kappa}$, a densidade de energia da radiação muda completamente seu comportamento com $\kappa$ para $a \gg a_{n}$, já que $\rho\left(a_{n}\right)=0\left(\rho^{\prime}\left(a \lesssim a_{n}\right)>0\right.$ ou $\left.\rho^{\prime}\left(a \lesssim a_{n}\right)<0\right)$ para uma geometria aberta (plana ou fechada, respectivamente). O valor de $a_{n}$ depende tanto do valor de $\mathcal{\kappa}$ como do valor de $n$.

\subsection{INTERVALO DE VALIDADE DO MODELO}

A continuação discutimos o intervalo de validade do modelo: 


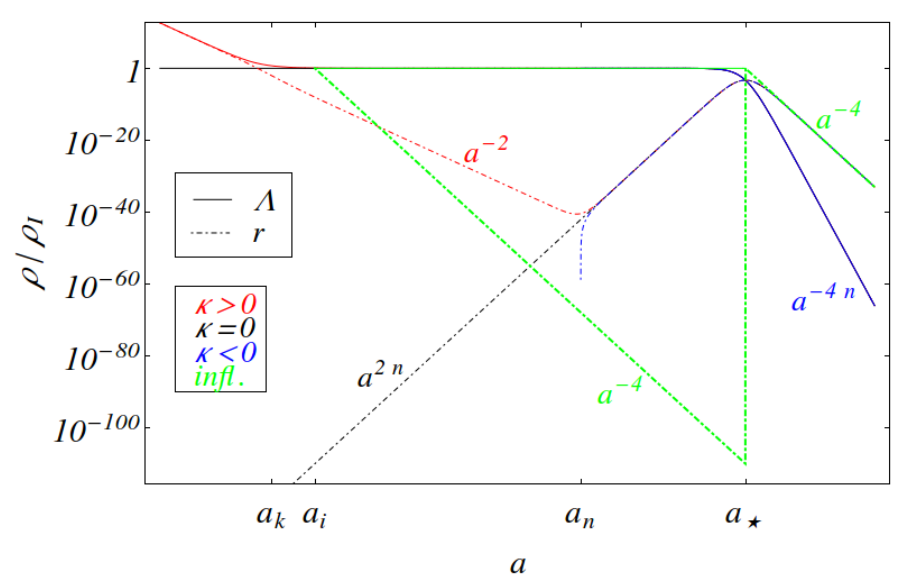

Figura 8.2: Evolução da densidade de energia da radiação durante a deflação com curvatura. Comparação entre a densidade de energia da radiação durante o processo deflacionário, e durante o processo inflacionário mais reaquecimento. O modelo inflacionário mais reaquecimento correspondem às curvas verdes, enquanto que o modelo deflacionário é representado pelas curvas vermelha, preta e azul, para as geometrias fechada, plana e aberta, repetitivamente. $a<a_{\kappa}$ corresponde ao período acelerado dominado pelo termo de curvatura na densidade do vácuo. $a_{i}$ corresponde ao limite superior do fator de escala tal que o modelo de inflação exponencial resolve o problema do horizonte. A validade do modelo deflacionário com $\kappa<0$ esta restrita a $a>a_{n}$, onde $a_{n}$ é tal que $\rho\left(a_{n}\right)=0$ neste modelo. $a_{\star}$ corresponde ao instante em que o parâmetro de desaceleração é nulo, no fim do período primordial acelerado.

- Na figura 8.1, as curvas pretas correspondem ao caso plano, em que o parâmetro de Hubble converge para $H_{I}$ quando $a \rightarrow 0$. A validade deste modelo (com $\kappa=0$ ) pode ser estendida indefinidamente para o passado, resolvendo o problema do horizonte.

- As curvas vermelhas na figura 8.1 representam o caso fechado, em que o parâmetro de Hubble se aproxima arbitrariamente de $H_{I}$ para $a \in\left(a_{k}, a_{\star}\right)$, mas sempre permanecendo menor do que ele; enquanto que para $a \ll a_{k}$, este converge para $H_{\star} \equiv H_{I} / 2^{n}$ e a densidade total escala como $\rho_{T} \propto \kappa / a^{2}$. A validade de este modelo esta limitada no passado ( $\operatorname{com} \kappa>0$ ) pela escala de energia de Planck, mas também consegue resolver o problema do horizonte, já que se $\rho_{I}$ for igual à escala de energia da grande unificação, termos que $a_{\kappa}<a_{i}$, e o modelo pode ser estendido até $a \lesssim a_{\kappa}$.

- Para $\kappa<0$ (representado por curvas azuis na figura 8.1), a validade do modelo é limitada no passado pela condição fraca de energia, isto é, pelo valor $a_{n}$ definido como o fator de escala tal que $\rho\left(a>a_{n}\right)>0$. Infelizmente este modelo, com $\kappa<0$, não resolve o problema do horizonte, já que $a_{n}>a_{i}$.

No modelo deflacionário com curvatura espacial $\kappa \geqslant 0$ as escalas que saíram do horizonte durante a primeira parte do período primordial acelerado, para $a<a_{\kappa}$, ainda não entraram no horizonte; e se o estágio atual acelerado persistir, ditas escalas nunca vão entrar no horizonte, deixando estas eras primordiais fora do nosso horizonte futuro de eventos. 


\section{COLAPSO GRAVITACIONAL COM UM VÁCUO DinÂMiCO E CURVATuRA ESPACIAL}

Além de afetar a evolução da historia cósmica, uma energia dinâmica do vácuo pode influenciar o processo de colapso gravitacional, porém, de uma forma diferente, já que, se para um universo em expansão a escala de energia tipica do sistema decresce junto com a densidade do vácuo, enquanto que durante o processo de colapso gravitacional estas duas quantidades crescem conforme o decorrer do tempo, produzindo efeitos opostos aos apresentados durante a expansão do universo.

Com isto em mente, podemos concluir que o efeito repulsivo devido à densidade do vácuo se incrementa durante o andamento do colapso gravitacional, entretanto, foi mostrado por [Campos and Lima, 2012] que uma densidade do vácuo que cresce de forma proporcional à densidade total do sistema não impede a formação um estado final singular, nem a formação de um horizonte aparente em volta de um núcleo estelar colapsante antes de atingir tal estado -no caso em que o sistema esfericamente simétrico, homogêneo e isotrópico é livre de curvatura espacial-.

No presente capítulo, vamos estudar os efeitos da curvatura espacial na formação de buracos negros a partir do colapso gravitacional de objetos compactos, ou seja, sem considerar o efeito de acreção de matéria. Para tal fim, vamos considerar o colapso de um núcleo estelar composto por uma mistura isotrópica e homogênea (esta aproximação deveria ser válida pelo menos durante os estágios avançados do colapso) de um fluido material e uma densidade de vácuo, que cresce de forma proporcional à densidade total -incluindo a densidade de curvatura espacial-. Esse último termo na densidade do vácuo (proporcional à densidade de curvatura) aumenta sua taxa de crescimento, se comparada com modelo sem curvatura [Campos and Lima, 2012]. Adicionalmente à hipótese de homogeneidade e isotropia do núcleo estelar, vamos assumir que o espaço-tempo interior é bem descrito por uma métrica espacialmente homogênea e isotrópica.

Ao longo do capítulo, vamos mostrar que a formação de uma singularidade e de um horizonte em volta dela não são afetados pelo sinal da curvatura espacial do sistema, no final, a curvatura não consegue impedir o crescimento sem limite da densidade total do sistema e da velocidade de colapso da casca externa, mas consegue modificar os valores do tempo de colapso, da massa colapsada e, consequentemente, do raio do horizonte aparente associado ao buraco negro formado [Perico et al., 2013b].

\subsection{DINÂMICA DO SISTEMA}

Para começar, vamos dividir o espaço-tempo em duas regiões que chamaremos como $V^{ \pm}$, onde $V^{+}$e $V^{-}$são a região exterior e interior do núcleo estelar, separadas pela 3 -superfície $\Sigma$. Uma vez que 
não estamos interessados no processo de acreção de matéria, a evolução da sub-variedade $V^{-}$não depende da métrica da sub-variedade exterior $V^{+}$. Além disso, a formação de um horizonte aparente para um sistema esfericamente simétrico somente depende da métrica e da distribuição de campos na sub-variedade interior. Por estas razões, no caso do estudo da formação de buracos negros, podemos restringir a nossa análise ao interior do núcleo estelar [Cai and Wang, 2006].

Para um núcleo estelar descrito pela métrica espacialmente homogênea e isotrópica:

$$
d s_{-}^{2}=g_{\mu v}^{-} d \chi_{-}^{\mu} d \chi_{-}^{v}=-d t^{2}+\mathrm{a}^{2}(t)\left(\frac{d r^{2}}{1-\kappa r^{2}}+r^{2} d \Omega^{2}\right)
$$

(onde $\kappa$ é o parâmetro de curvatura espacial enquanto que a é uma função do tempo) e composta pela mistura de um fluido material e do vácuo, as equações de Einstein ficam expressas como:

$$
\begin{gathered}
8 \pi G \rho+\Lambda(t)=3 \mathrm{H}^{2}+3 \frac{\tilde{\kappa}}{R^{2}} \\
8 \pi G \mathcal{P}-\Lambda(t)=-2 \frac{d \mathrm{H}}{d t}-3 \mathrm{H}^{2}-\frac{\tilde{\kappa}}{R^{2}},
\end{gathered}
$$

onde $\tilde{\kappa} \equiv \kappa r_{\Sigma}^{2}, R \equiv$ a $r_{\Sigma}$ é o raio físico do núcleo estelar, $\mathrm{H} \equiv(d R / d t) / R<0$ é a taxa de contração do núcleo e, $\rho$ e $\mathcal{P}$ são a densidade de energia e a pressão do fluido material com equação de estado

$$
\mathcal{P}=\omega \rho,
$$

onde $0 \leqslant \omega \leqslant 1$ é um parâmetro constante. Neste capítulo, vamos considerar uma densidade do vácuo como sendo descrita por:

$$
\Lambda=c_{0}+3 v\left(\mathrm{H}^{2}+\frac{\tilde{\kappa}}{R^{2}}\right),
$$

onde $v$ é um parâmetro adimensional. Essa é a generalização mais natural incluindo a curvatura espacial, da expressão (5.9) estudada em [Campos and Lima, 2012], e que produz a seguinte relação

$$
v=\frac{\rho_{\Lambda}-c_{0} / 8 \pi G}{\rho_{T}}
$$

que permanece constante ao longo do processo de colapso e generaliza a relação para um espaço-tempo plano proposta por [Carvalho et al., 1992], e derivada por [Shapiro and Solà, 2002; Solà, 2011] fazendo uso das propriedades gerais que devem possuir as equações efetivas do grupo de renormalização associadas ao valor esperado do vácuo.

Se usarmos as equações (9.2) e (9.4), podemos encontrar a equação que governa a evolução temporal do raio do núcleo colapsante:

$$
R \frac{d^{2} R}{d t^{2}}+\Delta\left(\frac{d R}{d t}\right)^{2}+\Delta \tilde{\kappa}-\frac{c_{0}}{2}(1+\omega) R^{2}=0
$$

onde $\Delta \equiv-1+3(1+\omega)(1-v) / 2$, e cuja primeira integral produz a expressão:

$$
\left(\frac{d R}{d t}\right)^{2}=b\left(\frac{R_{i}}{R}\right)^{2 \Delta}-\tilde{\kappa}+\frac{c_{0} R^{2}}{3(1-v)},
$$


onde

$$
b=\left(R_{i} \mathrm{H}_{i}\right)^{2}+\tilde{\kappa}-c_{0} \frac{R_{i}^{2}}{3(1-v)},
$$

é a constante de integração que foi fixada exigindo $\mathbf{H}\left(t_{i}\right) \equiv-\mathrm{H}_{i}$. Usando a solução (9.7) e as equações de Einstein (9.2) para escrever as densidades do vácuo e do fluido material em função de a, obtemos as seguintes expressões:

$$
\rho_{\Lambda}=\frac{v}{1-v}\left[\rho_{T}^{i}(1-v)-\frac{c_{0}}{8 \pi G}\right]\left(\frac{R_{i}}{R}\right)^{2 \xi}+\frac{1}{1-v} \frac{c_{0}}{8 \pi G}, \quad \rho=\left[\rho_{T}^{i}(1-v)-\frac{c_{0}}{8 \pi G}\right]\left(\frac{R_{i}}{R}\right)^{2 \xi}
$$

onde

$$
8 \pi G \rho_{T}^{i}=3 \mathrm{H}_{i}^{2}+3 \frac{\tilde{\kappa}}{R_{i}^{2}}=\frac{3 b}{R_{i}^{2}}+\frac{c_{0}}{(1-v)},
$$

é a densidade total no instante $t_{i}$, e $\xi \equiv 1+\Delta=3(1+\omega)(1-v) / 2$.
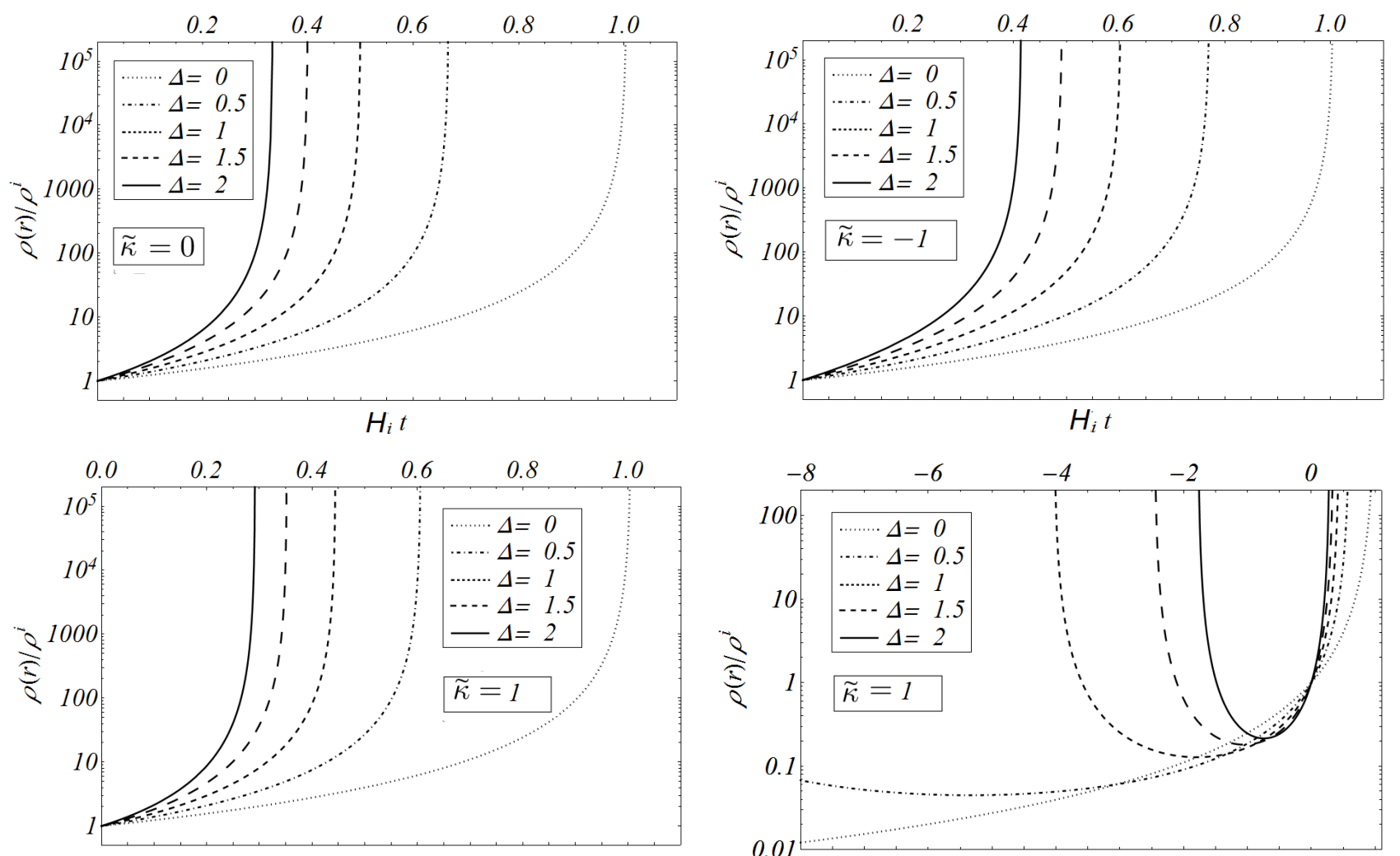

$H_{i} t$

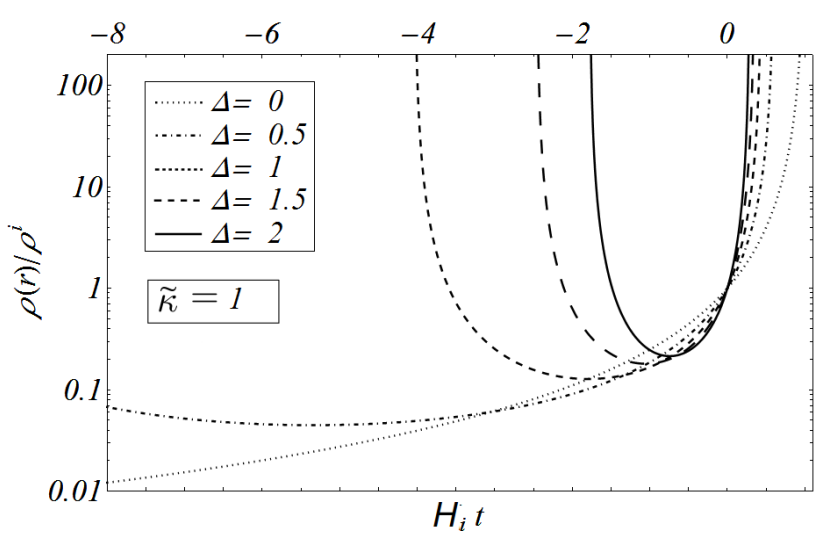

Figura 9.1: Densidade de energia material durante o colapso gravitacional no modelo $\Lambda=c_{0}+3 v\left(\mathrm{H}^{2}+\right.$ $\left.\tilde{\kappa} / R^{2}\right)$.

Podemos ver que para os caso de interesse físico $\Delta>0$ a densidade de energia do fluido material diverge para um valor finito de tempo (o tempo de colapso). Adicionalmente, o instante de tempo em que se atinge tal estado singular, partindo sempre das mesmas condições iniciais, acontece mais cedo (tarde) no caso da geometria fechada (aberta) se comparado com a geometria plana. Também a rapidez com a que aumenta a densidade de energia, e portanto diminui o tempo de colapso, é menor para valores menores de $v$ (sistemas com uma fração vácuo-matéria menor) já que $\Delta$ diminui com o aumento de $v$.

Na figura 9.1 se mostra a evolução da densidade de energia material em função do tempo adimensional $\mathrm{H}_{i}$ t para alguns valores do parâmetro $\Delta$ e do parâmetro de curvatura $\kappa$. Podemos ver que para todos os 
valores de $\Delta \geqslant 0$ a densidade de energia diverge quando se atinge o tempo de colapso $t_{c}$, que depende dos parâmetros $\Delta$ e $\kappa$. Também podemos ver que o tempo de colapso aumenta (diminui) no caso da geometria aberta (fechada) se comparado com o caso de geometria plana.

Dado que $c_{0} / 8 \pi G$ é muito menor que a densidade típica das estrelas (fenomenologicamente deveria ser próximo da estimativa cosmológica para a densidade do vácuo hoje), podemos desconsiderá-la durante os estágios avançados do colapso, nos que estamos interessados. Portanto, no que resta do capítulo vamos usar $c_{0}=0$. Com esta simplificação podemos usar a variável auxiliar

$$
u=\frac{\tilde{\kappa}}{b}\left(\frac{R}{R_{i}}\right)^{2 \Delta}
$$

para integrar a equação (9.7), que agora fica escrita como

$$
\frac{d u}{d t}=\frac{2 b^{1 / 2}(\tilde{\kappa} / b)^{A}}{(2 A-1) R_{i}} u^{1-A} \sqrt{1-u}
$$

onde $A=(1+\Delta) /(2 \Delta)$. Invertendo a equação (9.10), obtemos que

$$
\frac{d t}{d u}=\frac{(2 A-1) R_{i} b^{A-1 / 2}}{2 \tilde{\kappa}^{A}} \frac{u^{A-1}}{\sqrt{1-u}},
$$

e sua derivada pode ser escrita na forma

$$
u(1-u) \frac{d^{2} t}{d u^{2}}+\left\{(1-A)-\left(\frac{3}{2}-A\right) u\right\} \frac{d t}{d u}=0
$$

Essa equação é um caso particular da equação hipergeométrica com parâmetros $\alpha_{1}=0, \alpha_{2}=\frac{1}{2}-A$, e $\alpha_{3}=1-A$, e cuja solução é dada por [Abramowitz and Stegun, 1964]

$$
t(u)=c_{1}+c_{2} u^{A} F[1 / 2, A ; 1+A ; u],
$$

onde $c_{1}$ e $c_{2}$ são as constantes de integração, e $F\left[\alpha_{1}, \alpha_{2}, \alpha_{3}, u\right]$ é a função hipergeométrica de Gauss [Abramowitz and Stegun, 1964]. Considerando a condição inicial $T\left(t_{i}\right)=R_{i}$, e escolhendo $t(R=0) \equiv t_{c}$, podemos reescrever a solução acima como

$$
1-\frac{t}{t_{c}}=\left(\frac{R}{R_{i}}\right)^{1+\Delta} \frac{F\left[1 / 2, A ; 1+A ;\left(R / R_{i}\right)^{2 \Delta} \tilde{\kappa} / b\right]}{F[1 / 2, A ; 1+A ; \tilde{\kappa} / b]},
$$

onde o tempo de colapso $t_{c}$ é dado por:

$$
t_{c}=\mathrm{H}_{i}^{-1} \frac{F[1 / 2, A ; 1+A ; \tilde{\kappa} / b]}{(1+\Delta) \sqrt{1+\tilde{\kappa}\left(R_{i} \mathrm{H}_{i}\right)^{-2}}} .
$$

Sabendo que $F\left[\alpha_{1}, \alpha_{2}, \alpha_{3}, 0\right]=1$ para qualquer $\alpha_{i}$, obtemos que quando $\kappa \rightarrow 0$ a solução acima se reduz a

$$
\frac{R}{R_{i}}=\left(1-\frac{t}{t_{c}}\right)^{1 / \xi}, \quad t_{c}=\left(\mathrm{H}_{i} \xi\right)^{-1},
$$

que é o resultado obtido por [Campos and Lima, 2012] para o caso plano. 

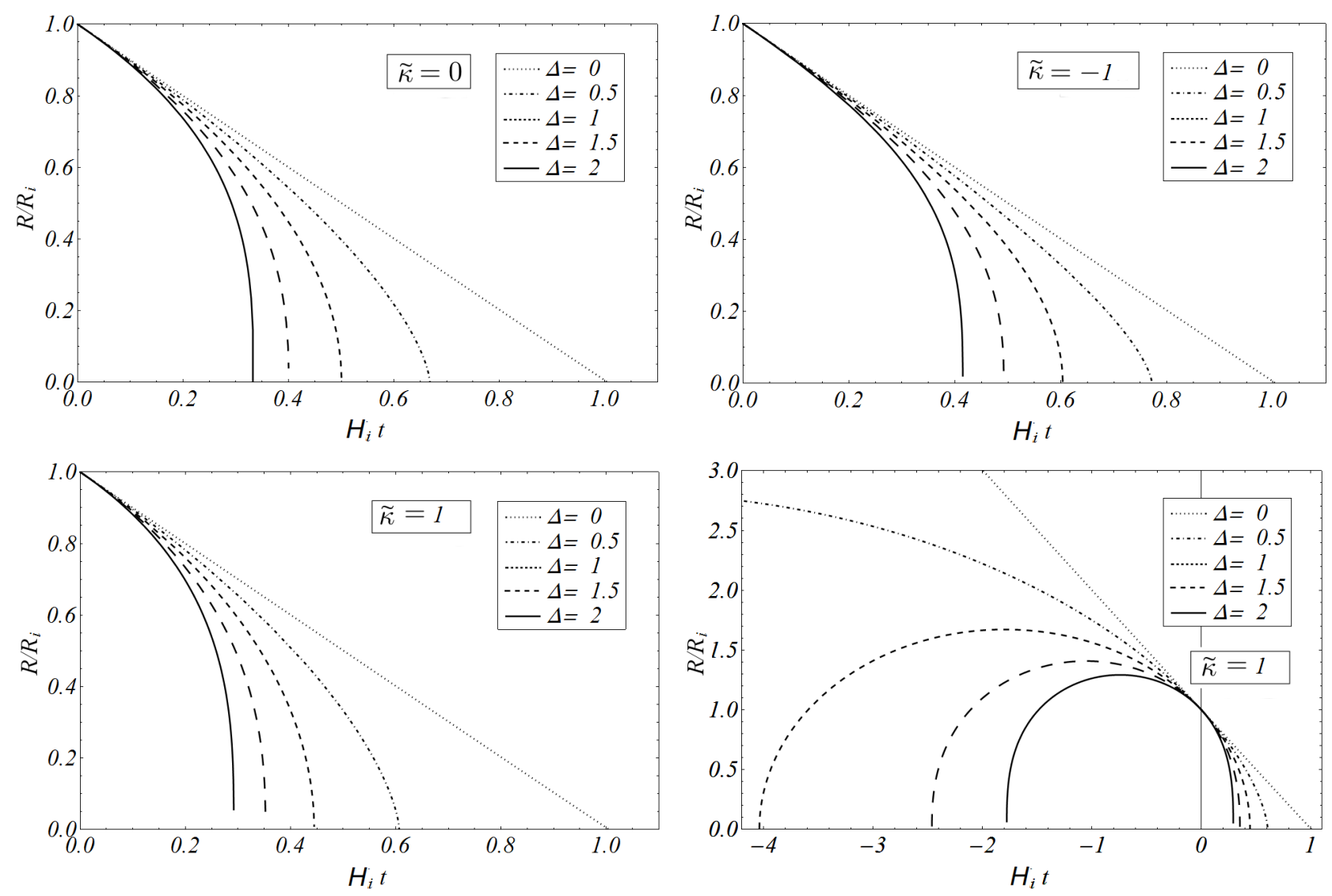

Figura 9.2: Evolução do raio de um sistema que colapsa no modelo com $\Lambda=c_{0}+3 v\left(\mathrm{H}^{2}+\kappa / \mathrm{a}^{2}\right)$.

Para os casos de interesse físico $\Delta>0$ podemos ver que o efeito do incremento na fração inicial de vácuo, para uma dada geometria (determinada pelo sinal de $\kappa$ ) se resume em retardar o tempo de colapso comparado com o caso em que o vácuo permanece constante $v=0$ (lembrando que $\Delta$ aumenta com a diminuição de $v$ ). Também, para um valor de $\Delta$ fixo, o colapso é favorecido pela geometria fechada $\kappa>0$ comparado com o caso plano, e no caso da geometria hiperbólica $\kappa<0$ o efeito é oposto. Adicionalmente, podemos ver que no caso em que $\kappa>0$ as configurações com $\Delta>0$ se expandem a partir de um estado singular, atingindo um raio máximo para depois colapsar e um estado singular; o processo oposto acontece no caso em que $\Delta<0$.

Na figura 9.2 se mostra a evolução de $R$ como função do tempo adimensional $H_{i} t$ para os três tipos de geometria e alguns valores característicos do parâmetro $\Delta$. Nota-se que para um valor fixo de $\Delta$, os modelos com curvatura positiva (negativa) apresentam um tempo de colapso menor (maior) comparados com o caso plano.

Como veremos a seguir, as soluções ficam um pouco mais simples a través da transformação $d t=a(\eta) d \eta$. Neste caso, a métrica (2.17) muda para

$$
d s^{2}=a^{2}(\eta)\left(d \eta^{2}-\frac{d r^{2}}{1-\kappa r^{2}}-r^{2} d \Omega^{2}\right)
$$

enquanto que a equação (9.6) fica escrita como:

$$
R \ddot{R}+(\Delta-1) \dot{R}^{2}+\kappa \Delta R^{2}=0 .
$$


Se fizermos a mudança de variável $z=R^{\Delta}$, a equação (9.18) toma a forma [Assad and Lima, 1988; Lima, 2001]:

$$
\ddot{z}+\kappa \Delta^{2} z=0 .
$$

A solução desta equação, após voltar para a variável $R$, pode ser escrita na forma:

$$
R(\eta)=\left\{c_{1} \sin [\eta \Delta \sqrt{\kappa}]+c_{2} \cos [\eta \Delta \sqrt{\kappa}]\right\}^{1 / \Delta}
$$

onde $c_{1}$ e $c_{2}$ são as constantes de integração, que podem ser determinadas impondo-se que a primeira derivada respeite a equação (9.7) e adicionalmente exigindo $R\left(\eta_{c}\right)=0$, com o que podemos reescrever a solução acima como:

$$
R(\eta)=R_{i}\left\{\sqrt{\frac{b}{\tilde{\kappa}}} \sin \left[\sqrt{\kappa} \Delta\left(\eta_{c}-\eta\right)\right]\right\}^{1 / \Delta}=R_{i}\left\{\frac{\sin \left[\sqrt{\kappa} \Delta\left(\eta_{c}-\eta\right)\right]}{\sin \left[\sqrt{\kappa} \Delta\left(\eta_{c}-\eta_{i}\right)\right]}\right\}^{1 / \Delta},
$$

onde o tempo conforme de colapso é dado por

$$
\eta_{c}-\eta_{i}=\frac{1}{\Delta \sqrt{\kappa}} \arcsin \left[\sqrt{\frac{\tilde{\kappa}}{b}}\right]=\frac{1}{\Delta \sqrt{\kappa}} \operatorname{arccot}\left[\frac{d R_{i}}{d t} \frac{1}{\sqrt{\tilde{\kappa}}}\right] .
$$

Substituindo-se (9.21) na equação (9.14), podemos obter a expressão para $t(\eta)$ e a função hipergeométrica nesta expressão pode ser simplificada para alguns valores particulares do parâmetro $\Delta$.

No caso em que $\Delta=1 / 2(\omega=v=0$ ou $\omega=2 v=1)$ obtemos a seguinte expressão, que ainda depende do parâmetro $\kappa$ :

$$
R(\eta)=R_{i} b\left(\frac{1}{\sqrt{\tilde{\kappa}}} \sin \left[\sqrt{\kappa}\left(\eta_{c}-\eta\right) / 2\right]\right)^{2}, \quad t_{c}-t=\frac{R_{i} b}{2 \tilde{\kappa} r_{\Sigma}}\left(\eta_{c}-\eta-\frac{1}{\sqrt{\kappa}} \sin \left[\sqrt{\kappa}\left(\eta_{c}-\eta\right)\right]\right),
$$

onde $\eta_{c}$ é dado pela equação (9.22) com $\Delta=1 / 2$. No caso da geometria fechada, plana ou aberta as expressões (9.22) e (9.23) se reduzem a:

(i) Geometria fechada:

$$
R(\eta)=R_{i} \frac{1-\cos \left[\eta_{c}-\eta\right]}{1-\cos \left[\eta_{c}-\eta_{i}\right]}, \quad t_{c}-t=R_{i} \frac{\left(\eta_{c}-\eta\right)-\sin \left[\eta_{c}-\eta\right]}{1-\cos \left[\eta_{c}-\eta_{i}\right]}, \quad \eta_{c}-\eta_{i}=2 r_{\Sigma} \operatorname{arccot}\left[\frac{d R_{i}}{d t}\right] .
$$

(ii) Geometria aberta:

$R(\eta)=R_{i} \frac{\cosh \left[\eta_{c}-\eta\right]-1}{\cosh \left[\eta_{c}-\eta_{i}\right]-1}, \quad t_{c}-t=R_{i} \frac{\sinh \left[\eta_{c}-\eta\right]-\left(\eta_{c}-\eta\right)}{\cosh \left[\eta_{c}-\eta_{i}\right]-1}, \quad \eta_{c}-\eta_{i}=2 r_{\Sigma} \operatorname{arccoth}\left[\frac{d R_{i}}{d t}\right]$.

(iii) Geometria plana:

$$
R(\eta)=R_{i}\left(\frac{\eta_{c}-\eta}{\eta_{c}-\eta_{i}}\right)^{2}, \quad t_{c}-t=\frac{R_{i}}{3} \frac{\left(\eta_{c}-\eta\right)^{3}}{\left(\eta_{c}-\eta_{i}\right)^{2}}, \quad \eta_{c}-\eta_{i}=2 r_{\Sigma}\left(\frac{d R_{i}}{d t}\right)^{-1},
$$


com o que, para $t_{i} \equiv 0$, podemos escrever:

$$
1-\frac{t}{t_{c}}=\left(\frac{R}{R_{i}}\right)^{3 / 2}
$$

como foi obtida na seção anterior (ver equação (9.16) $\operatorname{com} \xi=3 / 2$, é equivalente a escolher $\Delta=1 / 2$ ).

\subsection{FORMAÇÃO DO HORIZONTE APARENTE}

Uma componente essencial no estudo do processo de colapso gravitacional é a formação de um horizonte aparente, já que, se o estado final involucra uma singularidade, a conjectura da censura cósmica exige que esta esteja coberta por um horizonte aparente, proibindo singularidades visíveis por observadores no infinito espacial [Penrose, 2002]. Isto significa que quando um sistema colapsa atingindo uma singularidade no ponto $x$ no tempo de colapso $t_{c}$, um horizonte aparente em volta de $x$ deve ser formado em um tempo anterior a $t_{c}$.

Nosso objetivo na presente seção é estudar os efeitos de uma densidade do vácuo crescente da forma (9.4) durante o processo de colapso, na formação de um horizonte aparente. No caso simplificado, em que todas as variáveis no interior do núcleo estelar são homogêneas e isotrópicas, o horizonte aparente somente pode se formar na superfície da mesma. De acordo com [Santos, 1985], vamos definir a métrica intrínseca na superfície $\Sigma$ do corpo colapsante como:

$$
d s_{\Sigma}^{2}=g_{\mu \nu} d \xi^{\mu} d \xi^{v}=d t^{2}-R(t)^{2} d \Omega^{2}
$$

onde $R(t)=r_{\Sigma}$ a $(t)$ é o raio físico do núcleo estelar, de acordo com as condições de junção.

Como já vimos, o horizonte aparente de um sistema esfericamente simétrico deve ser uma 2-esfera do tipo nula. Dado que estamos interessados em condições iniciais tais que o sistema não possuiu um horizonte aparente no passado, assim, definimos as condições iniciais do nosso sistema tais que toda 2-esfera possua um vetor normal tipo tempo (tal que seja uma superfície tipo espaço como é o caso de toda 2-esfera no espaço-tempo de Minkowsky), e a formação de um horizonte aparente como a aparição de uma 2-esfera com vetor normal nulo [McVittie, 1933; Cahill and McVittie, 1970; Cai and Wang, 2006]. Adicionalmente, devido à homogeneidade e isotropia no interior do núcleo estelar, o horizonte aparente deve surgir justamente sobre a superfície da mesma, e para que o sistema forme um buraco negro, o horizonte aparente deve se formar antes que o sistema atinja uma singularidade.

Com tudo isso, a condição para a formação do horizonte aparente $(h)$ sobre a superfície do núcleo estelar colapsante, pode ser escrita nas coordenadas definidas pela métrica (9.24) como:

$$
\left.g^{\mu v} R_{, \mu} R_{, v}\right|_{h}=\left[\left(\frac{d R_{h}}{d t}\right)^{2}+\tilde{\kappa}\right]-1 \equiv 0,
$$



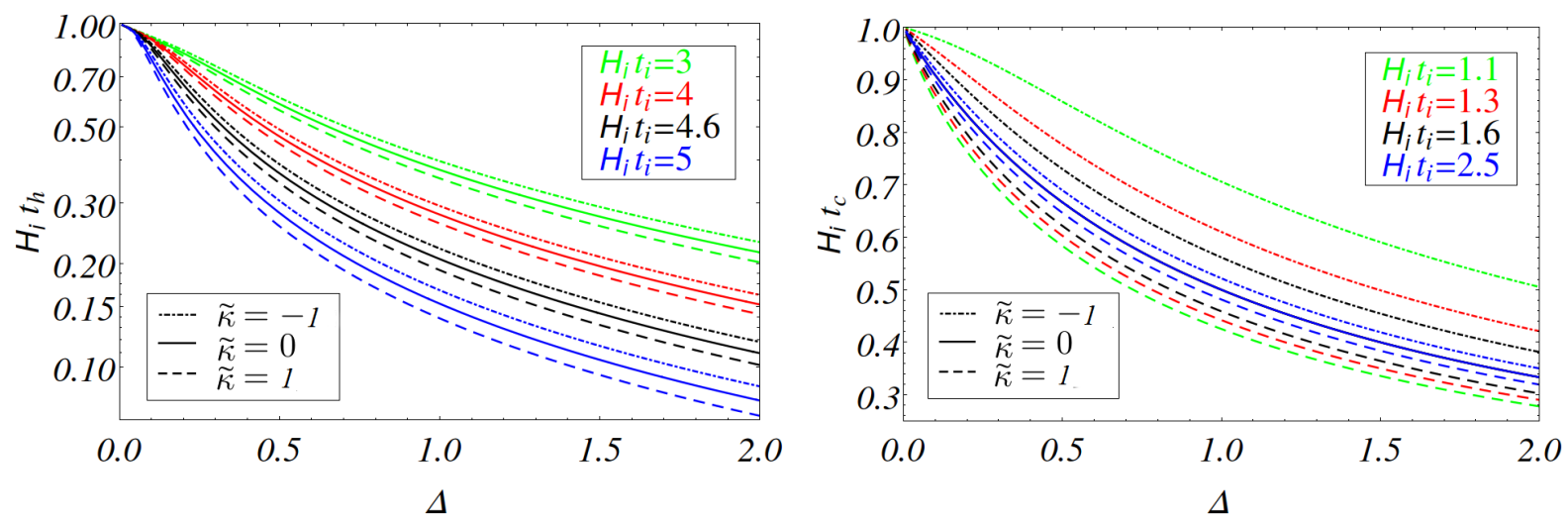

Figura 9.3: Tempo de formação do horizonte no processo de colapso com $\Lambda=c_{0}+3 v\left(\mathrm{H}^{2}+\tilde{\kappa} / R^{2}\right)$. Neste gráfico podemos ver que o tempo de formação do horizonte diminui quanto menor for a fração de vácuo presente no sistema (para valores maiores de $\Delta$ ). Adicionalmente o tempo de formação do horizonte se torna menor (maior) para a geometria fechada (aberta) se comparadas com o caso da geometria plana, como era de esperar-se já que a geometria fechada favorece o processo de colapso, diminuindo também o tempo de colapso.

Da mesma forma, as condições iniciais que definem a ausência de superfícies aparentes no interior so núcleo estelar ou sobre a sua superfície podem ser descritas por:

$$
\left.g^{\mu v} R_{, \mu} R_{, v}\right|_{i}=\left[\left(\frac{d R_{i}}{d t}\right)^{2}+\tilde{\kappa}\right]-1<0,
$$

o que implica em

$$
0<\left(\frac{d R_{i}}{d t}\right)^{2}+\tilde{\kappa}<1 .
$$

Nota-se que, para que não exista um horizonte aparente no interior do núcleo estelar ou na sua superfície no instante inicial, é suficiente e necessário que a condição (9.26), devido a que $\mid d($ a $r) / d t|<| d R / d t \mid$ para todo $r<r_{\Sigma}$.

Outra quantidade que podemos definir de forma covariante é a função massa, que fornece a massa gravitante dentro da esfera de raio $r$ no instante de tempo $t$ e que se reduz à massa do buraco negro de Schwarzschild no caso estático. No sistema de coordenadas (2.17), a função massa [Cahill and McVittie, 1970; Poisson and Israel, 1990; Wang et al., 1997; Villas da Rocha et al., 1999; Hayward, 2004] fica escrita da seguinte forma:

$$
m\left(t, r_{\Sigma}\right)=\frac{1}{2} R\left(1+g^{\mu v} R_{, \mu} R_{, v}\right)=\frac{1}{2} R\left[(R H)^{2}+\tilde{\kappa}\right] .
$$

Usando agora a solução (9.7) às equações de Einstein do sistema, podemos reescrever a condição para a formação do horizonte aparente na superfície do núcleo estelar como sendo:

$$
\left(\frac{d R_{h}}{d t}\right)^{2}+\tilde{\kappa}=b\left(\frac{R_{i}}{R_{h}}\right)^{2 \Delta}=1
$$


onde o sub-índice $h$ denota o instante de tempo quando o horizonte aparente é formado. Se agora usarmos a condição inicial (9.27), podemos ver que

$$
R_{h} \equiv R_{i}\left[\left(R_{i} \mathrm{H}_{i}\right)^{2}+\tilde{\kappa}\right]^{1 / 2 \Delta}<R_{i}, \quad \text { se } \quad \Delta>0,
$$

com isto podemos concluir que os casos de interesse físico, tais que não possuíram nenhum horizonte aparente para $R>R_{i}$, necessariamente são os casos com $\Delta>0$; portanto daqui para frente vamos trabalhar somente $\operatorname{com} \boldsymbol{\Delta}>\mathbf{0}$. Se agora usarmos a solução para a evolução temporal do raio do núcleo estelar (9.14), obtemos a seguinte relação entre o tempo de colapso $t_{c}$ e o tempo de formação do horizonte $t_{h}$ :

$$
\frac{t_{h}}{t_{c}}=1-\left(\frac{R_{h}}{R_{i}}\right)^{1+\Delta} \frac{F[1 / 2, A ; 1+A ; \tilde{\kappa}]}{F[1 / 2, A ; 1+A ; \tilde{\kappa} / b]} .
$$

No caso particular em que $\kappa=0$, a expressão acima se reduz à obtida por [Campos and Lima, 2012]:

$$
\frac{t_{h}}{t_{c}}=1-\left(R_{i} \mathrm{H}_{i}\right)^{1+1 / \Delta} .
$$

A Figura 9.3 mostra o comportamento do tempo adimensional $\mathrm{H}_{i} t_{h}$ de formação do horizonte e do tempo adimensional $\mathrm{H}_{i} t_{c}$ de colapso, em função do parâmetro $\Delta$ para os três tipos de geometria e diferentes condições iniciais.

Se agora avaliarmos a função massa (9.28) no instante de formação do horizonte aparente $h$, obtemos o valor da massa do buraco negro formado:

$$
m\left(\eta_{h}\right)=\frac{R_{h}}{2}=\frac{R_{i}}{2}\left[\left(R_{i} \mathrm{H}_{i}\right)^{2}+\tilde{\kappa}\right]^{1 / 2 \Delta}=m_{0}\left(\frac{\Omega^{i}}{1-v}\right)^{1 / 2 \Delta}
$$

onde $m_{0}=R_{i}\left(R_{i} \mathrm{H}_{i}\right)^{1 / \Delta} / 2$ é a massa do buraco negro no caso em que $\kappa=0$ [Campos and Lima, 2012] e $\Omega^{i}=8 \pi G \rho^{i} / 3 \mathrm{H}_{i}^{2}$. A expressão geral (9.33) pode ser reescrita em unidades solares como:

$$
M\left(t_{h}, r_{\Sigma}\right)=R_{i} \frac{M_{\odot}}{R_{S \odot}}\left[\left(R_{i} H_{i}\right)^{2} \frac{\Omega^{i}}{1-v}\right]^{1 / 2 \Delta}
$$

onde $M_{\odot}$ e $r_{S \odot}$ são a massa e o raio de Schuarzschild do sol. No caso plano, $\Omega^{i}=1-v$, a equação (9.34) se reduz a:

$$
M\left(\tau_{h}\right)=\frac{R_{i} M_{\odot}}{R_{S \odot}}\left(R_{i} \mathrm{H}_{i}\right)^{1 / \Delta}
$$

Se agora assumirmos que $R_{i}=80 \mathrm{Km}$ e $\left(R_{i} \mathrm{H}_{i}\right) \sim 1 / 2$ obtemos $M \sim 13.3 M_{\odot}$ para o caso de um núcleo estelar dominado por fluidos ultrarrelativísticos $(v=0, \omega=1 / 3)$. No entanto, para $v=0.25$ com os mesmo valores de $R_{i}$ e $\mathrm{H}_{i}$, se obtém valores menores para a massa, tipicamente da ordem de $M \sim 6.6 M_{\odot}$.

Com isso, podemos ver que a influência da curvatura espacial nos valores para a massa do buraco negro formado após o processo de colapso gravitacional são o aumento (diminuição) da massa colapsada no caso da geometria fechada (aberta) se comparados com o caso da geometria espacialmente plana no interior do núcleo estelar. 


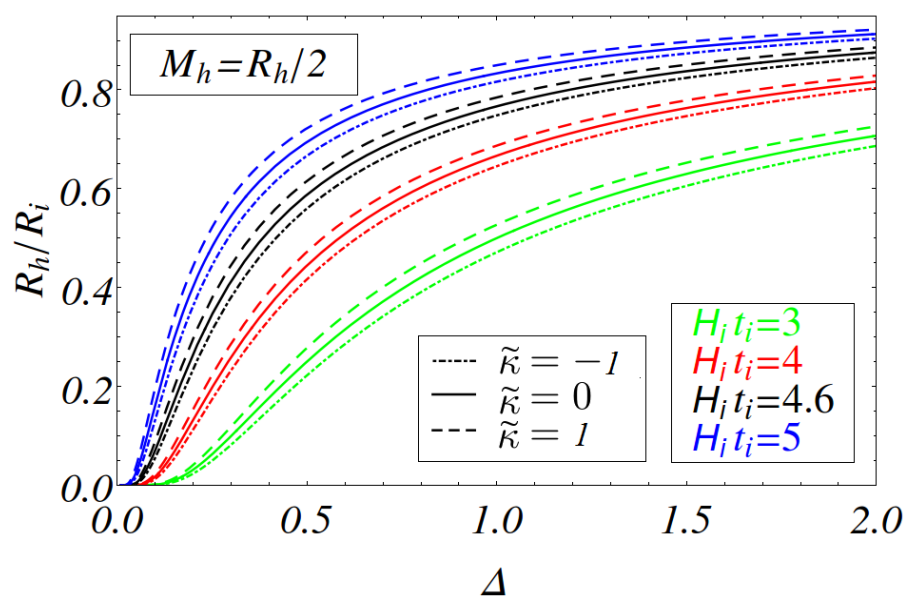

Figura 9.4: Massa do buraco negro formado no colapso gravitacional com $\Lambda=c_{0}+3 v\left(\mathrm{H}^{2}+\tilde{\kappa} / R^{2}\right)$. Para um valor fixo de $\mathrm{H}_{i}$ (condição inicial) podemos ver que a massa colapsada dentro do horizonte aparente é menor (maior) no caso da geometria aberta (fechada) se comparado com o caso da geometria plana. Adicionalmente podemos ver que para sistemas com uma fração inicial de vácuo maior, valores menores do parâmetro $\Delta$, a massa colapsada diminui.

\subsection{CONDIÇÕES DE JUNÇÃO}

Seguindo o procedimento feiro por Santos [Santos, 1985] vamos estabelecer as condições sobre as que podemos fazer a juntura entre as métricas que descrevem o interior e o exterior do núcleo estelar colapsante. Usando a métrica interna (9.1) (onde $B^{2}=\mathrm{a}^{2}(t) /\left(1-\kappa r^{2}\right)$ e $A=1$ se comparado com os resultados de [Santos, 1985], ver apêndice B.3) podemos obter o vetor normal à superfície do núcleo estelar (definida como $f(r, t)=r-r_{\Sigma}$, onde $r_{\Sigma}$ é uma constante):

$$
n_{\lambda}^{-}=\{0,1,0,0\} \frac{\mathrm{a}(t)}{\sqrt{1-\tilde{\kappa}}},
$$

com este podemos calcular a segunda forma fundamental sobre a superfície, definida como [Eisenhart, 1949; Santos, 1985]

$$
K_{\alpha \beta}^{ \pm}=-n_{\lambda}^{ \pm} \frac{\partial^{2} \chi_{ \pm}^{\lambda}}{\partial \xi^{\alpha} \partial \xi^{\beta}}-n_{\lambda}^{ \pm} \Gamma_{\mu v}^{\lambda} \frac{\partial \chi_{ \pm}^{\mu}}{\partial \xi^{\alpha}} \frac{\partial \chi_{ \pm}^{v}}{\partial \xi^{\beta}} .
$$

Em nosso caso obtemos as seguintes expressões para as componentes da segunda forma fundamental sobre a superfície do núcleo estelar, calculadas usando as coordenadas internas:

$$
K_{\tau \tau}^{-}=0, \quad K_{\theta \theta}^{-}=\left.R \sqrt{1-\tilde{\kappa}}\right|_{\Sigma}, \quad K_{\phi \phi}^{-}=\sin ^{2} \theta K_{\theta \theta}^{-} .
$$

Por outro lado, usando a métrica de Vaidya [Vaidya, 1951, 1953, 1966]:

$$
d s_{+}^{2}=g_{\mu v}^{+} d \chi_{+}^{\mu} d \chi_{+}^{v}=\left(1-\frac{2 m(v)}{\varangle}\right) d v^{2}+2 d v d r+r^{2} d \Omega
$$

para descrever o exterior do núcleo estelar colapsante, se obtém as seguintes expressões para as componentes da segunda forma fundamental:

$$
K_{\tau \tau}^{+}=\frac{v, \tau \tau}{v_{, \tau}}-\left.\frac{m v_{, \tau}}{\mathbb{r}^{2}}\right|_{\Sigma}, \quad K_{\theta \theta}^{+}=v, \tau\left(1-\frac{2 m}{r}\right) \mathbb{r}+\mathbb{r} r,\left.\tau\right|_{\Sigma}, \quad K_{\phi \phi}^{+}=\sin ^{2} \theta K_{\theta \theta}^{+} .
$$


Assumindo as seguintes condições de junção:

$$
\left.g_{\mu v}^{-}\right|_{\Sigma}=\left.g_{\mu v}^{+}\right|_{\Sigma},\left.\quad \quad K_{\alpha \beta}^{-}\right|_{\Sigma}=\left.K_{\alpha \beta}^{+}\right|_{\Sigma},
$$

temos que a massa de Vaidya se reduz a:

$$
m=\frac{R}{2}\left[(R H)^{2}+\tilde{\kappa}\right]
$$

é igual à massa de Cahill e McVittie (9.28), e representa a masa vista pelos observadores externos, descritos pela métrica de Vaidya. Adicionalmente as condições de junção produzem a restrição:

$$
0=2 \frac{\ddot{R}}{R}+\mathrm{H}^{2}+\frac{\tilde{\kappa}}{R^{2}},
$$

que comparada com as equações de Einstein no interior do núcleo estelar (9.2) produz a seguinte restrição sobre o conteúdo energético do sistema:

$$
\mathcal{P}_{\text {tot }}=0
$$

A maneira mais simples de obter um sistema com uma pressão total $\mathcal{P}_{\text {tot }}=0$ é considerar a presencia simultânea de matéria ultrarrelativística e matéria escura, contribuindo separadamente para a densidade do vácuo da seguinte forma:

$$
\dot{\rho}_{r}+3 H\left(\rho_{r}+\mathcal{P}_{r}\right)=-\dot{\rho}_{v r}, \quad \dot{\rho}_{m}+3 H\left(\rho_{m}+\mathcal{P}_{m}\right)=-\dot{\rho}_{v m},
$$

onde $\rho_{v r}$ e $\rho_{v m}$ são as contribuições individuais da matéria ultrarrelativísticos e da matéria à energia do vácuo. A solução a estas duas equações pode ser escrita como:

$$
\begin{gathered}
\rho_{m}=\rho_{m}^{i}\left(\frac{R}{R_{i}}\right)^{-2 \xi_{m}}, \quad \rho_{r}=\rho_{r}^{i}\left(\frac{R}{R_{i}}\right)^{-2 \xi_{r}}, \\
\rho_{v} \equiv \rho_{v r}+\rho_{v r}=\rho_{v}^{i}+\frac{v_{r} \rho_{r}^{i}}{1-v_{r}}\left[\left(\frac{R}{R_{i}}\right)^{-2 \xi_{r}}-1\right]+\frac{v_{m} \rho_{m}^{i}}{1-v_{m}}\left[\left(\frac{R}{R_{i}}\right)^{-2 \xi_{m}}-1\right],
\end{gathered}
$$

onde $\xi_{r}=2\left(1-v_{r}\right)$ e $\xi_{m}=3\left(1-v_{m}\right) / 2$. Neste caso, a condição de pressão nula produz as seguintes expressões:

$$
\frac{v_{r}}{1-v_{r}} \rho_{r}^{i}+\frac{v_{m}}{1-v_{m}} \rho_{m}^{i}=\rho_{v}^{i}=\frac{1}{3} \rho_{r}^{i}, \quad \text { and } \quad \xi_{r}=\xi_{m},
$$

cuja solução representa o seguinte vínculo para os parâmetros $v_{r}$ e $v_{m}$ :

$$
1=4 v_{r}-3 v_{m}
$$

No caso mais geral, onde $\mathcal{P} \neq 0$, as condições de junção obtidas por Santos [Santos, 1985] (para a métrica externa de Vaidya [Vaidya, 1953]) requerem a inclusão de um termo de fluxo de calor ao longo da direção radial dentro do tensor momento energia total do sistema, e que não pode ser mais descrito por uma métrica interna homogênea e isotrópica da forma (9.1). 


\subsection{RESUMO DO CAPÍtULO}

Neste capítulo estudamos o processo de colapso de um núcleo estelar massivo cujo conteúdo esta formado por uma mistura de um fluido simples descrito pela equação de estado $\mathcal{P}=\omega \rho, \omega=$ cte, e um termo de vácuo da forma $\Lambda(H, a)=3 v H^{2}+3 v \tilde{\kappa} / R^{2}$ [Carvalho et al., 1992; Shapiro and Solà, 2002; Solà, 2011].

Para um conjunto de condições iniciais e parâmetros livres fixos, o processo de colapso é favorecido pela geometria fechada, se comparado com o caso da geometria plana, enquanto a geometria aberta apresenta os efeitos opostos.

Encontramos que os casos de interesse físico, sistemas que não apresentam horizontes aparentes no passado, se restringem aos casos com $\Delta>0$. Sobre tais condições, a densidade total sempre se incrementa durante o processo de colapso até atingir uma singularidade em um tempo finito $t_{c}$, atingindo a condição de formação do horizonte aparente para um tempo $t_{h}$ anterior ao tempo de colapso.

Adicionalmente, para o caso da geometria fechada, o sistema admite uma solução que parte de um estado singular em expansão, atinge um raio físico máximo e depois colapsa em um buraco negro.

Finalmente, enfatizamos que a maior simplificação do modelo de colapso esfericamente simétrico foi assumir que o espaço-tempo no interior pode ser modelado como sendo homogêneo e isotrópico, o que poderia ter validade nos estágios avançados do processo.

A principal conclusão é que uma energia do vácuo proporcional à densidade total não impede a formação de uma singularidade ao incluir os efeitos da densidade de curvatura, mas foi possível quantificar seus efeitos no atraso (adiantamento) da formação do horizonte e da singularidade, como o decréscimo (aumento) da massa colapsada no caso da geometria fechada (aberta) comparados com o caso plano. Uma conclusão, derivada diretamente dos resultados de Santos [Santos, 1985] para o caso homogêneo e isotrópico no interior do núcleo estelar, é a requerimento da nulidade da pressão total sobre a superfície do sistema. Esta condição pode ser satisfeita se o sistema é constituído por matéria ultrarrelativística e matéria escura, se as duas contribuem separadamente para a densidade do vácuo, com uma fração específica, tal que a pressão da matéria ultrarrelativística anula a pressão do vácuo total (vácuo da matéria ultrarrelativística mais vácuo da matéria escura). Em qualquer outro caso, para que o sistema possa ser descrito pela métrica externa de Vaidya [Vaidya, 1953], o tensor momento energia total do conteúdo energético interior deve possuir um termo de fluxo de calor radial [Santos, 1985], ou, a densidade total do núcleo estelar deve diminuir continuamente com a distância ao centro até se anular na superfície. Em qualquer um dos dois casos, a subvariedade interna do sistema não pode ser descrita por uma métrica homogênea. 


\section{COLAPSO GRAVITACIONAL COM VÁCUO DINÂMICO}

Neste capítulo estudamos os efeitos de uma densidade do vácuo que cresce mais rapidamente que a densidade de energia do fluido material no regime de altas energias, dominando os estágios finais do processo de colapso gravitacional de um núcleo estelar esfericamente simétrico, homogêneo e isotrópico. Estamos particularmente interessados nos estágios finais do colapso, que podem ser caracterizados pela formação, ou não, de um horizonte aparente e de uma singularidade. Devido ao domínio do vácuo durante o estágio final do processo de colapso, este acontece de forma desacelerada, e a princípio prolonga-se por um intervalo de tempo infinito, enquanto a densidade total do sistema evolui assintoticamente para um valor constante e finito, evitando a formação de uma singularidade e levando ao sistema para uma fase final estacionária.

Adicionalmente, durante o processo de colapso, dependendo das condições iniciais e do valor dos parâmetros livres do modelo, o sistema pode ou não formar um horizonte aparente que esconde o estado final -não singular- para os observadores externos, mas sempre forma um horizonte so tipo de Sitter associado com o estágio final exponencialmente desacelerado.

\subsection{GEOMETRIA E COMPOSIÇÃO DO SISTEMA}

Como foi feito no capítulo 9, vamos centrar nossa análise no interior do núcleo estelar colapsante, já que se um sistema gravitacional esfericamente simétrico gera um horizonte aparente, este vai ser formado dentro ou sobre sua superfície, e sua formação não depende da geometria externa nem das condições de junção entre as métricas que descrevem o espaço-tempo interior e exterior.

Nosso seguinte passo é considerar o interior do núcleo estelar como sendo homogêneo, isotrópico (o que é uma aproximação razoável durante os estágios avançados do processo de colapso) e com um raio finito. Sobre estas condições o espaço-tempo interior é bem descrito pela métrica escrita em coordenadas esféricas:

$$
d s^{2} \equiv d t^{2}-\mathrm{a}^{2}(t)\left(d r^{2}+r^{2} d \theta^{2}+r^{2} \sin \theta d \phi^{2}\right),
$$

onde $R \equiv\left(\right.$ a $\left.r_{\Sigma}\right)$ é o raio físico do núcleo estelar. Assumindo que os campos materiais e o vácuo são comoveis no espaço-tempo descrito pela métrica (10.1), podemos definir a superfície do núcleo estelar $\Sigma$-para qualquer instante de tempo- por seu raio comóvel, que denotaremos como:

$$
\left.r\right|_{\Sigma} \equiv r_{\Sigma}=\text { cte }
$$




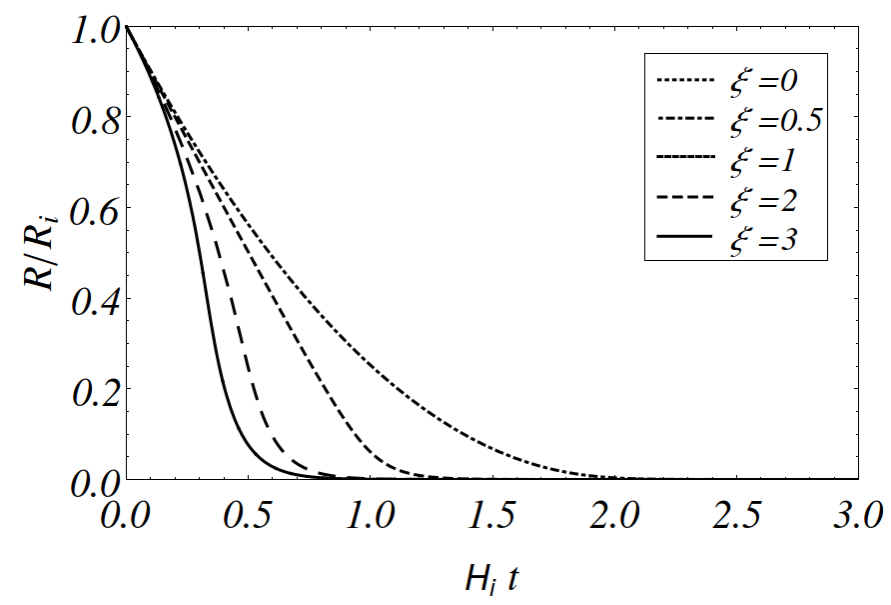

Figura 10.1: Raio do núcleo estelar durante os estágios avançados do colapso com $\Lambda=c_{0}+3 v \mathrm{H}^{2}+\gamma \mathrm{H}^{4}$. Escolhemos a origem do tempo $t_{i}$ tal que $R\left(t_{i}\right) \equiv R_{i}$ já dentro do estágio intermediário do colapso. Se $\xi>1$ o colapso acontece aceleradamente (devido ao domínio do fluido material) durante o estágio intermediário; no caso oposto o colapso acontece desaceleradamente durante dito estágio. Já durante o estágio final do colapso (quando o termo $\mathrm{em} \mathrm{H}^{4}$ se torna dominante) o sistema é sempre dominado pela energia do vácuo levando o sistema a um período perpetuo de colapso desacelerado. Quando o parâmetro $\mathrm{H}_{f}$ é finito, o raio do núcleo vai para zero somente no limite $t=t_{c} \rightarrow \infty$.

Por outro lado, vamos assumir una densidade do vácuo que evolui da forma dada pelos primeiros termos da expansão 5.14:

$$
8 \pi G \rho_{\Lambda}(t)=\Lambda(t)=c_{0}+3 v \mathrm{H}^{2}+\gamma \mathrm{H}^{4},
$$

onde $c_{0}, v$ e $\gamma$ são parâmetros constantes positivos, enquanto que a taxa de contração $\mathrm{H}$ é definida como $\mathrm{H} \equiv(d R / d t) / R$. Adicionalmente, os parâmetros $1 \gg v \gg \gamma c_{0}$, o que faz com que a densidade do vácuo seja dominada por $c_{0}$ para baixas energias, por $\gamma \mathrm{H}^{4}$ para altas energias e por $\nu \mathrm{H}^{2}$ para energias intermediárias. Esta expressão é proposta como solução à equação do grupo de renormalização para o valor esperado do vácuo expandida como serie de potências em $\mathrm{H}^{2}$, onde os coeficientes dos termos proporcionais a $\mathrm{H}^{2 n}$ são da ordem de $\left(M_{X}\right)^{-n}$ (ver seção 6.4.1). $M_{X}$ é da ordem da massa dos campos mais pesados da teoria quântica de campos usada para descrever as interações não gravitacionais.

No caso do modelo padrão das partículas elementares a escala $M_{X}$ é usualmente proposta como sendo a escala de energia da grande unificação ou inclusive a escala de energia de Planck. Portanto os termos de ordem superior na expansão em serie de potências em $\mathrm{H}^{2}$, para a densidade do vácuo, são desconsiderados na presente abordagem, e ainda obtemos $c_{0} \ll \gamma H_{i}^{4}$ para o coeficiente do termo proporcional a $\mathrm{H}^{4}$.

Assumindo a equação de estado $\mathcal{P}_{\Lambda}(t)=-\rho_{\Lambda}(t)$ para a densidade do vácuo $(10.3)$, e $\mathcal{P}(t) / \rho(t)=$ $\omega=$ cte. para o fluido material (onde $\rho$ e $\mathcal{P}$ são sua densidade de energia e sua pressão, com $\omega \geqslant 0$ ) as equações de Einstein para a métrica (10.1) são descritas pelas expressões (2.44), e junto com a expressão 

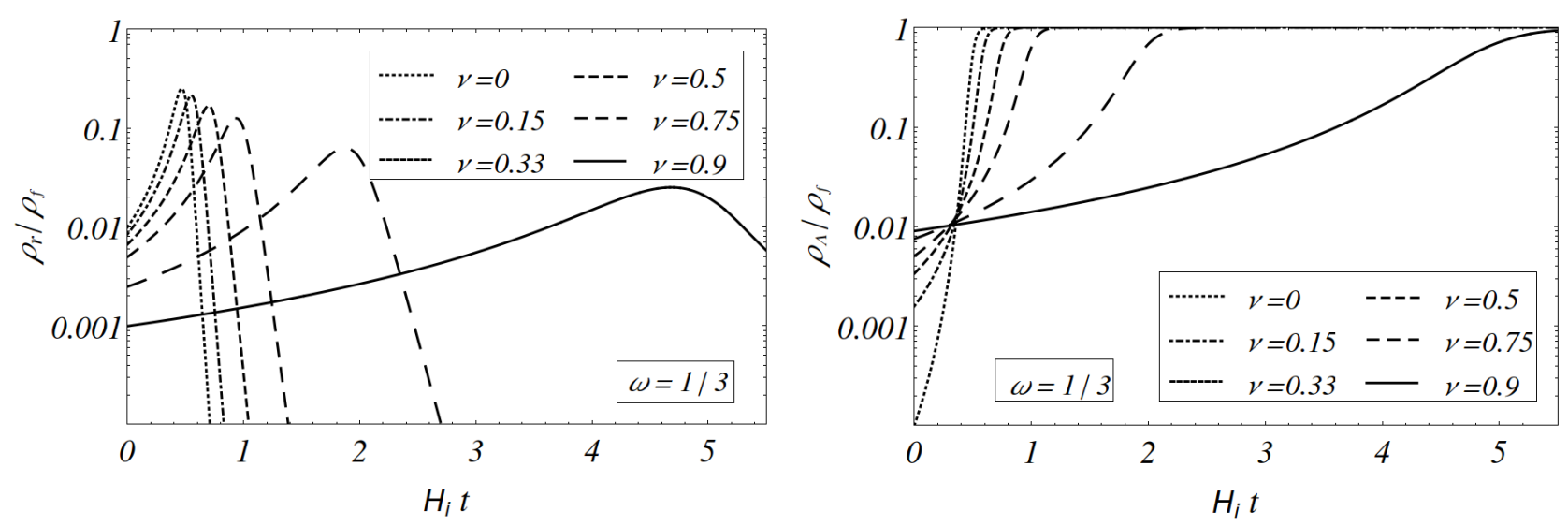

Figura 10.2: Evolução das densidades nos estágios avançados do colapso com $\Lambda=c_{0}+3 v \mathrm{H}^{2}+\gamma \mathrm{H}^{4}$. Comparando as duas figuras, podemos ver que o sistema é dominado pelo vácuo durante o período intermediário nos casos em que $v>1 / 2$, ou pelo fluido material se $v<1 / 2$. Adicionalmente, durante o estágio final do colapso a densidade do vácuo tende para um valor constante independentemente dos valores de $v$ e do parâmetro $\omega$ (ou seja, independentemente do fluido que domine durante os estágio intermediário), enquanto que a densidade de energia do fluido material apresenta um comportamento decrescente, permitindo o domínio do vácuo durante o estágio final.

para a densidade do vácuo (10.3) permitem obter a seguinte equação para a evolução temporal da taxa de contração $\mathrm{H}<0$ associado ao sistema colapsante:

$$
\frac{d \mathrm{H}}{d t}-\xi \mathrm{H}^{2}\left(\frac{\mathrm{H}_{i}^{2}}{\mathrm{H}^{2}}-1+\frac{\mathrm{H}^{2}}{\mathrm{H}_{f}^{2}}\right)=0,
$$

onde $\mathrm{H}_{f} \equiv \sqrt{3(1-v) / \gamma}$ é um parâmetro positivo da ordem de $M_{X}^{1 / 2}, \mathrm{H}_{i} \equiv \sqrt{c_{0} /(3(1-v))}$ é também positivo e fenomenologicamente da ordem de $\mathrm{H}_{i} \leqslant \mathrm{H}_{0} \lll \mathrm{H}_{f}$, enquanto que

$$
\xi \equiv \frac{3}{2}(1+\omega)(1-v)
$$

é também um parâmetro positivo. Nota-se que $\xi<3 / 2$ para poeira $(\omega=0)$ e $\xi<2$ para matéria ultrarrelativística $(\omega=1 / 3)$.

Tomado o limite $\mathrm{H}^{2} \ll \mathrm{H}_{f}^{2}$ a equação (10.4) apresenta a solução estacionária $\mathrm{H}=-\mathrm{H}_{i}<0$. Similarmente para $\mathrm{H}^{2} \gg \mathrm{H}_{i}^{2}$ esta equação possui a solução estacionária $\mathrm{H}=-\mathrm{H}_{f}<0$. Isso nos permite definir três estágios para a evolução temporal de $\mathrm{H}$. O estágio inicial do processo de colapso, quando $\mathrm{H}^{2} \ll \mathrm{H}_{f}^{2}$, corresponde a um estágio caracterizado pelo afastamento lento na magnitude de $|\mathrm{H}|$ do seu limite inferior $\mathrm{H}_{i}$. Quando $\mathrm{H}$ se afasta o suficiente de $\mathcal{O}\left(-\mathrm{H}_{i}\right)$, o termo proporcional a $\mathrm{H}^{2}$ se torna importante produzindo um incremento na taxa de crescimento do modulo de $\mathrm{H}$; chamaremos este período de estágio intermediário. Por fim, chamaremos de estágio final ao período do processo de colapso em que $\mathrm{H}^{2} \gg \mathrm{H}_{i}^{2}$; durante este período a evolução do sistema é dominada pelo termo proporcional a $\mathrm{H}^{4}$, levando assintoticamente $\mathrm{H}$ a seu valor limite $-\mathrm{H}_{f}<-\mathrm{H}_{i}<0$.

Dado que nosso principal interesse é analisar a influência da densidade do vácuo dinâmica (10.3) na formação de buracos negros, vamos centrar nosso estudo nos estágios avançados do processo de colapso (estágio intermediário e estágio final, segundo a convenção que definimos no parágrafo anterior). Por 
tanto, no que resta deste capítulo vamos desconsiderar a contribuição da constante $\mathcal{c}_{0}$ escolhendo nossa origem temporal $t_{i}$ já dentro do estágio intermediário do colapso. Como consequência vamos ter que $\Lambda=3 v \mathrm{H}^{2}+\gamma \mathrm{H}^{4}$, e a equação diferencial que dita a evolução temporal do sistema (10.4) para $t>t_{i}$ torna-se:

$$
\frac{d \mathrm{H}}{d t}+\xi \mathrm{H}^{2}\left(1-\frac{\mathrm{H}^{2}}{\mathrm{H}_{f}^{2}}\right)=0 .
$$

Integrando a equação acima obtemos:

$$
\mathrm{H}(R)=-\mathrm{H}_{f}\left\{1+\varrho_{1}\left(\frac{R}{R_{i}}\right)^{2 \xi}\right\}^{-1 / 2},
$$

onde a constante de integração $\varrho_{1}$ pode ser fixada como sendo $\varrho_{1} \equiv\left(\mathrm{H}_{f}^{2}-\mathrm{H}_{i}^{2}\right) / \mathrm{H}_{i}^{2}$ ao impor a condição inicial $\mathrm{H}\left(R_{i}\right) \equiv-\mathrm{H}_{i}<0$. Integrando mais uma vez esta equação podemos obter a seguinte relação entre o raio do núcleo estelar e o tempo:

$$
\varrho_{2}-\xi \mathrm{H}_{f} t=\sqrt{1+\varrho_{1}\left(\frac{R}{R_{i}}\right)^{2 \xi}}-\operatorname{arccoth}\left[\sqrt{1+\varrho_{1}\left(\frac{R}{R_{i}}\right)^{2 \xi}}\right],
$$

onde a nova constante de integração $\varrho_{2} \equiv\left(\mathrm{H}_{f} / \mathrm{H}_{i}-\operatorname{arccoth}\left[\mathrm{H}_{f} / \mathrm{H}_{i}\right]\right)$ foi ajustada escolhendo a origem do tempo como $R\left(t_{i}\right)=R_{i}$ (lembrando que $t=t_{i}$ já dentro do estágio intermediário do colapso). Nesta solução podemos ver que o espaço-tempo não apresenta uma singularidade final para valores finitos do parâmetro $\mathrm{H}_{f}$. Em breve veremos que esta solução implica um estágio final desacelerado, evitando a formação de uma singularidade, devido a que a velocidade de queda de todas as camadas da esfera colapsante diminui proporcionalmente à distância física ao centro da estrela, durante o estágio final assintoticamente estacionário (o que pode ser visto a partir da equação (10.7): $\partial_{t}(a r) \approx-\mathrm{H}_{f}($ ar ) quando $($ ar $) \rightarrow 0)$.

Substituindo a solução (10.7) nas equações de Einstein (10.4), podemos obter as seguintes expressões para as densidades de energia do vácuo e do fluido material:

$$
\rho_{\Lambda}=\rho_{T}^{f} \frac{1+v \varrho_{1}\left(R / R_{i}\right)^{2 \xi}}{\left(1+\varrho_{1}\left(R / R_{i}\right)^{2 \xi}\right)^{2}}, \quad \rho=\rho_{T}^{f} \frac{(1-v) \varrho_{1}\left(R / R_{i}\right)^{2 \xi}}{\left(1+\varrho_{1}\left(R / R_{i}\right)^{2 \xi}\right)^{2}}
$$

onde $\rho_{T}^{f} \equiv 3 \mathrm{H}_{f}^{2} / 8 \pi G$ é a densidade total quanto $R \rightarrow 0$ (quanto $t \rightarrow \infty$ ).

Nas figuras 10.2 podemos ver a evolução na densidade de energia do vácuo e do fluido material (poeira ou matéria ultrarrelativística). Nestas figuras podemos notar que durante o estágio final do colapso o sistema torna-se dominado pela densidade do vácuo, enquanto $\mathrm{H}$ atinge assintoticamente seu valor terminal $-\mathrm{H}_{f}$. Em outras palavras, o colapso atinge assintoticamente uma era de Sitter no estágio final do processo, que carece de uma singularidade, coincidindo com os resultados de [Cai and Wang, 2006] para um colapso dominado por uma componente de energia escura.

Examinando a equação (10.9) podemos notar que a densidade de energia do fluido material $\rho$ atinge um valor máximo quando $R=R_{i} \varrho_{1}^{-1 / 2 \xi}$. Vamos usar este instante de tempo como o tempo característico entre o estágio intermediário (onde $d \rho / d t>0$, ou $R>R_{i} \varrho_{1}^{-1 / 2 \xi}$ ) e o estágio final (onde $d \rho / d t<0$, ou $\left.R<R_{i} \varrho_{1}^{-1 / 2 \xi}\right)$. 


\subsubsection{Estágio intermediário do processo de colapso}

Durante o estágio intermediário do colapso, a evolução do sistema pode ser descrita pelas soluções (10.8-10.9) com $R \gg R_{i} \varrho_{1}^{-1 / 2 \xi}$. Com isto, o raio do núcleo estelar $R$, e as densidades de energia do vácuo e do fluido material se reduzem a:

$$
\left(\varrho_{2}-\xi \mathrm{H}_{f} t\right)^{2} \approx \varrho_{1}\left(\frac{R}{R_{i}}\right)^{2 \xi}, \quad \rho_{\Lambda} \approx v \rho_{T}^{i}\left(\frac{R_{i}}{R}\right)^{2 \xi}, \quad \rho \approx(1-v) \rho_{T}^{i}\left(\frac{R_{i}}{R}\right)^{2 \xi}
$$

onde $\rho_{T}^{i} \equiv 3 \mathrm{H}_{i}^{2} /\left(8 \pi G \varrho_{3}\right)$ é a densidade total para $t=0$ (lembrando que definimos $R(t=0) \equiv R_{i}$ já dentro do estágio intermediário, onde assumimos que o espaço tempo dentro do núcleo estelar pode ser descrito como boa aproximação pela métrica homogênea e isotrópica (10.1)), e $\varrho_{3} \equiv\left(\mathrm{H}_{f}^{2}-\mathrm{H}_{i}^{2}\right) / \mathrm{H}_{f}^{2}=$ $\varrho_{1} \mathrm{H}_{i}^{2} / \mathrm{H}_{f}^{2} \lesssim 1$.

Durante o estágio intermediário (onde $d \rho / d t>0$ ) as densidades de energia do vácuo e do fluido material apresentam uma evolução similar (ver as figura 10.2), as duas densidades se incrementam, de acordo com as expressões (10.10), enquanto que o raio físico do núcleo estelar decresce.

Por outro lado, as equação de Einstein (2.44) dizem para nós que

$$
-\frac{d^{2} R}{d t^{2}}=\frac{8 \pi G R}{6}\left(\rho_{T}+3 \mathcal{P}_{T}\right)
$$

onde $\rho_{T}$ e $\mathcal{P}_{T}$ são a densidade de energia e a pressão total do sistema interno. A expressão acima, durante o estágio intermediário, se escreve como

$$
-\frac{d^{2} R}{d t^{2}}=R \frac{\mathrm{H}_{i}^{2}}{\varrho_{3}}\left(\frac{R_{i}}{R}\right)^{2 \xi}(\xi-1),
$$

se usarmos as expressões (10.10). Usando este resultado e o fato de que para um processo de colapso $\mathrm{H}<0$ obtemos que: durante o estágio intermediário o sistema colapsa aceleradamente se $v<(1+$ $3 \omega) /(3+3 \omega)$ (o que é equivalente a $\xi>1$ ), os desaceleradamente no caso oposto.

Se o estágio intermediário é acelerado $(\xi>1)$, e persiste suficiente tempo, ou seja, se $\left(R / R_{i}\right)^{2 \xi} \varrho_{1} \ll 1$ inclusive para valores pequenos do raio $R$ (o que acontece se $\mathrm{H}_{f}^{2} \gg \mathrm{H}_{i}^{2}$ ), a densidade de energia dos dois fluidos consegue crescer muito (junto com $\left(\partial_{t} R\right)^{2}$ ) produzindo um objeto pequeno e denso o suficiente para gerar um horizonte aparente em torno dele.

Por outro lado, no caso em que $\mathrm{H}_{f} \rightarrow \infty$ o processo de colapso acaba durante o estágio intermediário, as soluções (10.10) definem a evolução do sistema até atingir o colapso se substituindo as constantes $\varrho_{3} \rightarrow 1$ e $\varrho_{2} \rightarrow H_{f} / H_{i}$ (o que corresponde às soluções de [Campos and Lima, 2012]). Neste limite, a expressão para a evolução do raio físico do núcleo estelar (10.10) assume a forma simples (9.16) obtida no capítulo anterior para $\kappa \rightarrow 0$ :

$$
\left(1-\xi \mathrm{H}_{i} t\right)=\left(\frac{R}{R_{i}}\right)^{1 \xi}
$$

onde podemos ver que para $\xi>1$ o colapso permanece acelerado, portanto atingindo um estado final singular em um lapso de tempo finito $t_{c}=1 / \mathrm{H}_{i} \xi$ como obtido previamente por [Campos and Lima, 2012] (ver também (9.16)). 

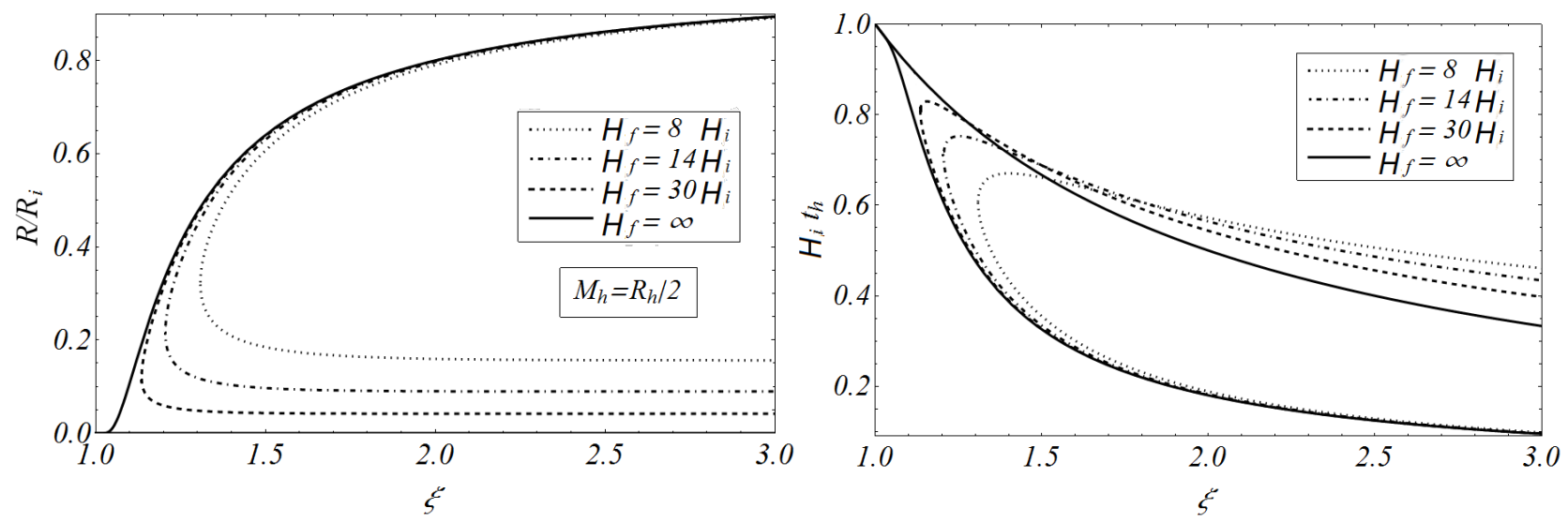

Figura 10.3: Formação dos horizontes aparentes no colapso gravitacional com $\Lambda=c_{0}+3 v \mathrm{H}^{2}+\gamma \mathrm{H}^{4}$. Painel Esquerdo: A parte superior (inferior) de cada curva representa o raio físico do núclelo estelar quando o horizonte externo (interno) é formado, para um dado valor de $\xi$. No caso em que $\mathrm{H}_{f} \rightarrow \infty$ o horizonte interno se torna uma singularidade. Painel Direito: A figura representa o instante de formação dos horizontes externo (parte inferior de cada curva) e interno (parte superior de cada curva). No caso em que $\mathrm{H}_{f} \rightarrow \infty$ a parte inferior da curva representa o tempo de colapso; lembrando que para $\mathrm{H}_{f}$ finito, o tempo de colapso diverge.

\subsubsection{Estágio de Sitter final}

Durante o estágio final do colapso, quando $\varrho_{1}\left(R / R_{i}\right)^{2 \tilde{\zeta}} \ll 1$ e $d \rho / d t<0$, as soluções gerais (10.8-10.9) podem ser aproximadas por

$$
R \approx R_{\circledast} e^{\mathrm{H}_{f}\left(t_{\circledast}-t\right)}, \quad \rho_{\Lambda} \approx \rho_{T}^{f}\left\{1+4(v-2)\left(\frac{R}{R_{\circledast}}\right)^{2 \xi}\right\}, \quad \rho \approx 4 \rho_{T}^{f}(1-v)\left(\frac{R}{R_{\circledast}}\right)^{2 \xi},
$$

onde $t_{\circledast} \equiv\left(\varrho_{2}-1\right) /\left(\mathrm{H}_{f} \xi\right)$, e $\left(R_{\circledast} / R_{i}\right)^{2 \xi} \equiv 4 / \varrho_{1}$. As soluções acima e as figura 10.2 mostram que durante o estágio final a densidade do vácuo atinge assintoticamente o valor terminal $\rho_{f}$, enquanto que a densidade de energia do fluido material diminui exponencialmente em função do tempo. Estas duas coisas fazem com que o sistema seja dominado pelo vácuo, levando-lhe para uma era de Sitter desacelerada durante um estágio final perpétuo.

Como é natural, a era de Sitter está acompanhada de um horizonte. No caso em que o sistema forma dois horizontes ou um horizonte, sobre a superfície do núcleo estelar durante os estágios intermediário e final (ver a figuras 10.4 e 10.5, e a equação (10.29)), o horizonte de Sitter corresponde ao segundo ou único horizonte formado (parte inferior das curvas na figura 10.3). No entanto, quando nenhum horizonte é formado dentro ou sobre a superfície do núcleo estelar durante os estágios avançados do colapso, o horizonte de Sitter ainda existe (devido à natureza exponencialmente desacelerada do colapso), mas esta localizado fora da superfície do núcleo estelar, por esta ração o gráfico 10.3 não revela sua existência.

Nota-se que o estágio final do colapso não converge à formação de uma singularidade, e isto ocorre devido à diminuição exponencial da velocidade de queda física das camadas esféricas do núcleo estelar colapsante, enquanto a energia total atinge assintoticamente um valor finito $\rho_{f}$. Em outras palavras, o 
estágio final do colapso é estacionário. A característica estacionária desde estágio pode ser posta em evidência fazendo a seguinte mudança coordenada:

$$
\begin{aligned}
t & \rightarrow t+\mathrm{H}_{f}^{-1} \ln \left[\cosh \left(r \mathrm{H}_{f}\right)\right], \\
r & \rightarrow \mathrm{H}_{f}^{-1}\left(\frac{r_{\Sigma}}{R_{\circledast}}\right) e^{\mathrm{H}_{I}\left(t-t_{\circledast}\right)} \sinh \left(r \mathrm{H}_{f}\right), \\
\theta & \rightarrow \theta \\
\phi & \rightarrow \phi,
\end{aligned}
$$

com o que a métrica (10.1) torna-se independente do tempo:

$$
d s^{2}=\frac{1}{\cosh ^{2}\left(r \mathrm{H}_{f}\right)}\left(d t^{2}-d r^{2}-\frac{\sinh ^{2}\left(r \mathrm{H}_{f}\right)}{\mathrm{H}_{f}^{2}} d \Omega^{2}\right) .
$$

\subsection{EStágios aCelerados e Desacelerados}

Após o estágio inicial do colapso gravitacional, quando $\mathrm{H}^{2} \gg c_{0}$, a equação (10.4) pode ser simplificada a

$$
\frac{d^{2} R}{d t^{2}}=R\left(\frac{R \mathrm{H}}{r_{\Sigma}}\right)^{2}\left[1-\xi\left(1-\frac{\mathrm{H}^{2}}{\mathrm{H}_{f}^{2}}\right)\right],
$$

e possui a solução (10.7), que tem a propriedade $\mathrm{H}^{2} \leqslant \mathrm{H}_{f}^{2}$. Podemos ver na equação acima, lembrando que $\mathrm{H}<0$, que para $\xi<1$ o processo de colapso é sempre desacelerado, e assim o sistema atinge um volumem nulo somente em um intervalo de tempo infinito, sem formar um horizonte aparente dentro ou sobre a superfície do núcleo estelar. No entanto, existindo um horizonte de Sitter fora do núcleo.

Por outro lado, para $\xi>1$ (ou seja, se $v<1 / 2$ no caso da matéria ultrarrelativística, ou $v<1 / 3$ no caso da matéria) a solução (10.7) permite as seguintes opções:

$$
\begin{array}{lll}
\frac{d^{2} R}{d t^{2}}<0 & \text { para } & R>R_{c}, \\
\frac{d^{2} R}{d t^{2}}>0 & \text { para } & R<R_{c}, \quad \text { período acelerado },
\end{array}
$$

onde o valor crítico $R_{c}$ é:

$$
R_{c} \equiv \frac{R_{i}}{\left(\varrho_{1}(\xi-1)\right)^{1 / 2 \xi}} \gtrless R_{i}, \quad \text { se }\left(\frac{\mathrm{H}_{f}}{\mathrm{H}_{i}}\right)^{2} \lessgtr \frac{\xi}{\xi-1},
$$

assim, para $\mathrm{H}_{f}^{2} / \mathrm{H}_{i}^{2}<\xi /(\xi-1)$ os estágios avançados são desacelerados. E para $\mathrm{H}_{f}^{2} / \mathrm{H}_{i}^{2}>\xi /(\xi-1)$ o estágio intermediário acontece de forma acelerada, mas para $R<R_{c}$ o colapso torna-se desacelerado, impedindo a formação de uma singularidade.

Nota-se que quando $\mathrm{H}_{f} \rightarrow \infty$ temos que $R_{c} \rightarrow 0$, portanto o estágio desacelerado nunca acontece, levando aceleradamente o sistema a um estado singular em um tempo finito. Assim o processo de colapso acaba durante o estágio intermediário em um intervalo de tempo finito, como obtido previamente por [Campos and Lima, 2012]. 


\subsection{HORIZONTE APARENTE}

Um horizonte aparente $(\mathscr{T})$ no caso de um sistema colapsante esfericamente simétrico é formado quando uma superfície esférica usual no sentido Minkowskiano, ou seja tipo espaço, de raio $R$ torna-se uma superfície nula [Cai and Wang, 2006] por causa da alta densidade de energia dentro dela, o que acontece quando

$$
\left.g^{\mu v} R_{, \mu} R_{, v}\right|_{h}=\stackrel{\circ}{R}^{2}-1=0,
$$

onde $R(t)=r_{\Sigma}$ a $(t)$ é o raio geométrico da 2-esfera definida por $\left\{t, r_{\Sigma}\right\}=$ constantes, $t_{h}$ é definido como o instante em que é formado o horizonte aparente, $\Sigma$ denota a superfície do núcleo estelar colapsante, e o anel ${ }^{\circ}$ denota a derivada em relação ao tempo. Usando a solução (10.7) a condição acima para a formação do horizonte aparente pode ser reescrita como:

$$
\stackrel{R}{h}_{h}^{2}=\frac{\left(R_{h} \mathrm{H}_{f}\right)^{2}}{1+\varrho_{1}\left(R_{h} / R_{i}\right)^{2 \xi}} \equiv 1 .
$$

Já que no presente capítulo estamos interessados em estudar a formação de horizontes durante o processo de colapso gravitacional de um núcleo estelar, vamos impor que no instante inicial $\left(t=t_{i}\right)$ não exista nenhum horizonte aparente ao interior do núcleo ou sobre a sua superfície (equivale a que a superfície do núcleo é tipo espaço no instante inicial):

$$
\left.R_{, \mu} R_{, v} g^{\mu v}\right|_{i}=\stackrel{\circ}{R}_{i}^{2}-1<0,
$$

$\mathrm{ou}$

$$
-1<\stackrel{\circ}{R}_{i}<0 .
$$

No entanto, durante o andamento do colapso podem surgir horizontes aparentes sobre ou dentro da superfície do núcleo estelar, o que depende das condições iniciais e dos valores dos parâmetros livres do modelo como veremos em breve.

\subsubsection{Quantos horizontes aparentes podem se formar}

O problema de encontrar o numero de soluções físicas para a condição de formação de um horizonte aparente na superfície do núcleo estelar (soluções reais da equação $(10.21) \stackrel{R}{h}_{h}^{2} \equiv 1$ ) pode ser reduzido a mostrar que a função $\stackrel{\circ}{R}^{2}(R)$ possui um máximo absoluto nos casos em que $\xi>1$, enquanto que apresenta um comportamento monótono se $\xi \leqslant 1$.

- Para $\xi \leqslant 1$ temos que $d \AA^{2} / d R>0$ para todo $R<R_{i}$ (ver equação (10.24)), assim a função $R^{2}$ decresce monotonamente do seu valor inicial $\stackrel{\circ}{R}^{2}\left(t_{i}\right)=\stackrel{\circ}{R}_{i}^{2}<1$ até o valor $\stackrel{\circ}{R}^{2}(R \rightarrow 0)=0$ como é mostrado na figura 10.4. Portanto concluímos que nenhum horizonte aparente é formado na superfície do núcleo estelar para $t>0$ no caso em que $\xi \leqslant 1$.

- Agora, para $\xi>1$ temos que

$$
\frac{d \stackrel{R}{R}^{2}}{d R}=2 \frac{d^{2} R}{d t^{2}}=2 R \mathrm{H}_{f}^{2} \frac{1-(\xi-1) \varrho_{1}\left(R / R_{i}\right)^{2 \xi}}{\left(1+\varrho_{1}\left(R / R_{i}\right)^{2 \xi}\right)^{2}}
$$




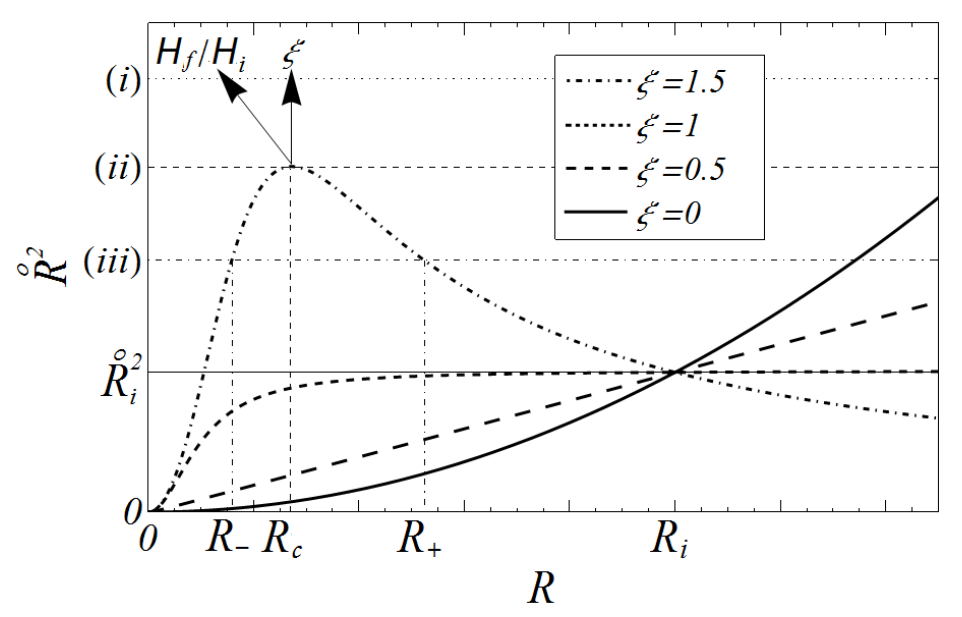

Figura 10.4: Evolução da função $\AA^{2}$ durante o processo de colapso com $\Lambda=3 v \mathrm{H}^{2}+\gamma \mathrm{H}^{4}$. Para $\xi \leqslant 1$ a função $\stackrel{\circ}{R}^{2}$ decresce monotonamente do seu valor inicial $\stackrel{\circ}{R}_{i}^{2}<1$ até $\stackrel{\circ}{R}^{2} \rightarrow 0$ (quando $R \rightarrow 0$ ), portanto, sem atingir a condição para a formação de um horizonte aparente. Para $\xi>1 \mathrm{e}$ $\mathrm{H}_{f}^{2} / \mathrm{H}_{i}^{2}>\xi /(\xi-1)$ (ou seja, para $R_{i}>R_{c}$ ) a função $\stackrel{\llcorner}{R}^{2}$ possui um máximo absoluto em $R_{c} \in\left(0, R_{i}\right)$, o que possui ainda 3 casos diferentes: $(i)$ para $\stackrel{\circ}{R}_{c}<1$ nenhum horizonte é formado durante o processo de colapso, (ii) para $\stackrel{\circ}{c}_{c}^{2}=1$ se forma um horizonte quando $R=R_{c}$, e (iii) para $\stackrel{\circ}{c}_{c}^{2}>1$ dois horizontes são formados em $R_{+}>R_{c}$ e $R_{-}<R_{c}$. Para $\xi>1$ as setas mostram a direção em que o máximo da função $\AA^{2}$ é puxado quando tomamos valores maiores nos parâmetros $\mathrm{H}_{f} / \mathrm{H}_{i}$ e $\xi$. Finalmente, para $\xi>1$ e $\mathrm{H}_{f}^{2} / \mathrm{H}_{i}^{2}<\xi /(\xi-1)$ o máximo da função $\stackrel{R}{R}^{2}$ acontece para $R_{c}>R_{i}$, assim a função $\dot{R}^{2}$ decresce monotonamente para $R<R_{c}$ e portanto o núcleo estelar não forma nenhum horizonte sobre a sua superfície ou dentro dela.

e

$$
\frac{d \dot{R}^{2}}{d R}\left\{\begin{array}{rll}
\rightarrow 0 & \text { quando } & R \rightarrow 0 \\
>0 & \text { quando } & R \in\left(0, R_{c}\right) \\
=0 & \text { quando } & R=R_{c} \\
<0 & \text { quando } & R \in\left(R_{c}, \infty\right) \\
\rightarrow 0 & \text { quando } & R \rightarrow \infty
\end{array}\right.
$$

onde o valor crítico $R_{c}$ é dado por (10.19). Similarmente, a segunda derivada da função $\AA^{2}$ é

$$
\frac{d^{2} \stackrel{R}{R}^{2}}{d R^{2}}=\frac{2 \mathrm{H}_{f}^{2}}{(1+\chi)^{3}}\left(c_{a} \chi^{2}+c_{b} \chi+1\right),
$$

onde $c_{a}=(\xi-1)(2 \xi-1), c_{b}=-(2 \xi-1)(\xi+2)$ e $\chi \equiv \varrho_{1}\left(R / R_{i}\right)^{2 \xi}$. Esta segunda derivada possui duas raízes reais $R_{-}<R_{c}$ e $R_{+}>R_{c}$ (ver figura 10.4), dadas por:

$$
R_{ \pm}=R_{c}\left\{1+\frac{\xi}{2} \pm \frac{\xi}{2} \sqrt{(\xi+2)^{2}-4 \frac{\xi-1}{2 \xi-1}}\right\}^{1 / 2 \xi}
$$


com isto obtemos que

$$
\frac{d^{2} \stackrel{\circ}{R}^{2}}{d R^{2}}\left\{\begin{array}{lll}
>0 & \text { quando } & R \in\left[0, R_{-}\right) \\
=0 & \text { quando } & R=R_{-} \\
<0 & \text { quando } & R \in\left(R_{-}, R_{+}\right) \\
=0 & \text { quando } & R=R_{+} \\
>0 & \text { quando } & R \in\left(R_{+}, \infty\right) \\
\rightarrow 0^{+} & \text {quando } & R \rightarrow \infty,
\end{array}\right.
$$

e sabendo que $\stackrel{\circ}{R}^{2} \rightarrow 0$ quando $R \rightarrow\{0, \infty\}$ podemos concluir que para $\xi>1$ a função $\stackrel{\circ}{R}^{2}$ possui um máximo absoluto em $R=R_{c}$.

Vamos agora nos concentrar no caso em que $\xi>1$, em tal caso a função $\stackrel{\circ}{R}^{2}(R)$ possui um máximo absoluto em $R=R_{c}$, o que ainda permite duas possibilidades:

- Para $\stackrel{\circ}{R}_{i}^{2}<1$ (estado inicial sem horizontes aparentes) com $R_{i}<R_{c}$ (é valido se $\mathrm{H}_{f}^{2} / \mathrm{H}_{i}^{2}<$ $\xi /(\xi-1))$, devido a que $d \stackrel{R}{R}^{2} / d R=2 d^{2} R / d t^{2}>0$ para $R<R_{c}$ obtemos que a função $\stackrel{R}{ }^{2}$ decresce assintoticamente até zero sem formar nenhum horizonte aparente.

- Para um estado inicial sem horizontes aparentes $\stackrel{\circ}{R}_{i}^{2}<1$ mas com $R_{i}>R_{c}$ (o que é válido se $\left.\mathrm{H}_{f}^{2} / \mathrm{H}_{i}^{2}>\xi /(\xi-1)\right)$ obtemos que $(i)$ no caso em que $\stackrel{R}{R}_{c}^{2}>1$ o sistema forma dois horizontes durante o processo de colapso; (ii) se $\stackrel{\circ}{R}_{c}^{2}=1$ o sistema forma um único horizonte, e (iii) se $\stackrel{\circ}{R}_{c}^{2}<1$ o sistema não forma nenhum horizonte (ver a figura 10.4$). \stackrel{\circ}{R}_{c}^{2} \equiv \stackrel{\circ}{R}^{2}\left(R_{c}\right)$ é dado por

$$
\stackrel{\circ}{R}_{c}^{2}=\stackrel{\circ}{R}_{i}^{2} \frac{\mathrm{H}_{f}^{2}}{\mathrm{H}_{i}^{2}} \frac{(\xi-1)^{1-1 / \xi}}{\xi \varrho_{1}^{1 / \xi}} .
$$

Agora, impondo que $\stackrel{\circ}{R}_{c}^{2} \lesseqgtr 1$ para $\xi>1$ podemos escrever a condição inicial $\stackrel{\circ}{R}_{i}^{2}$, em função dos parâmetros do modelo $\left\{\xi, \mathrm{H}_{f} / \mathrm{H}_{i}\right\}$, para que o sistema forme 2, ou 1 ou nenhum horizonte dentro ou sobre sua superfície para algum $t>0\left(\right.$ se $R_{c}<R_{i}$, o que acontece se $\left.\mathrm{H}_{f}^{2} / \mathrm{H}_{i}^{2}>\xi /(\xi-1)\right)$ :

$$
\stackrel{\circ}{R}_{i}^{2}\left\{\begin{array}{cl}
<\stackrel{\circ}{R}_{c i}^{2} & , \text { não forma horizontes } \\
=\stackrel{\circ}{R}_{c i}^{2} & , \text { forma } 1 \text { horizonte } \\
>\stackrel{\circ}{R}_{c i}^{2} & , \text { forma } 2 \text { horizontes }
\end{array}\right.
$$

onde o valor crítico para o parâmetro inicial $\stackrel{\circ}{R}_{c i}^{2}$ depende de $\mathrm{H}_{f} / \mathrm{H}_{i}$ e $\xi$ da forma:

$$
\stackrel{\circ}{R}_{c i}^{2}=\xi(\xi-1)^{(1-\xi) / \xi} \frac{\mathrm{H}_{i}^{2}}{\mathrm{H}_{f}^{2}}\left(\frac{\mathrm{H}_{f}^{2}}{\mathrm{H}_{i}^{2}}-1\right)^{1 / \xi} .
$$

Fisicamente, parâmetros tais que o sistema é dominado pelo vácuo $(\xi \leqslant 1)$ durante o estágio intermediário não formam horizontes aparentes, devido a que o processo de colapso acontece sempre desaceleradamente (começando com $-1<\stackrel{R}{R}<0$ e evoluindo monotonamente até $\stackrel{R}{R} \rightarrow 0^{-}$), e portanto sem atingir nunca a condição de formação de um horizonte aparente.

Os sistemas dominados por o fluido material durante o estágio intermediário $(\xi>1)$ e com uma condição inicial próxima da condição para a formação de um horizonte aparente $\stackrel{\circ}{R}_{i}^{2}>\stackrel{\circ}{R}_{c i}^{2}\left(\mathrm{H}_{f} / \mathrm{H}_{i},,_{\zeta}\right)$ 


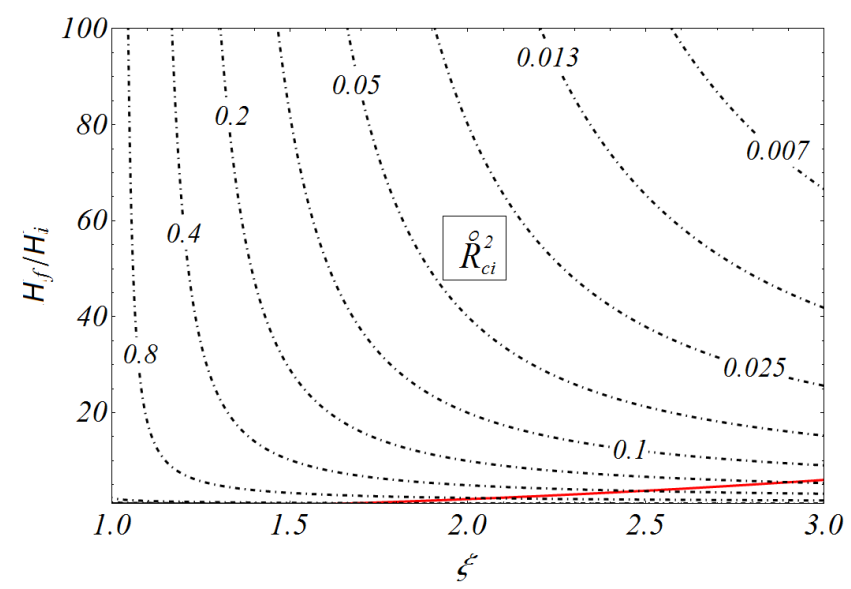

Figura 10.5: Espaço de parâmetros com 2, 1 ou 0 horizontes no colapso com $\Lambda=3 v \mathrm{H}^{2}+\gamma \mathrm{H}^{4}$. Se mostra os contornos da função $\dot{R}_{c i}^{2}$, Eq. (10.30), no espeço dos parâmetros $\left\{\tilde{\xi}, \mathrm{H}_{f} / \mathrm{H}_{i}\right\}$. Assim, para uma dada condição inicial, por exemplo $\stackrel{\circ}{R}_{i}^{2}=0.2$, os sistemas com parâmetros por baixo do contorno $\stackrel{\circ}{R}_{c i}^{2}=0.2$ permitem a formação de dois horizontes; os sistemas com coordenadas (no espaço dos parâmetros) sobre este contorno permitem a formação de um horizonte; e os sistemas com parâmetros por cima do contorno não permitem a formação de horizontes aparentes. A linha contínua representa o valor mínimo de $\mathrm{H}_{f}^{2} / \mathrm{H}_{i}^{2}$ em que o máximo da função $\stackrel{\stackrel{R}{ }}{2}^{2}$ ocorre para $R_{c}<R_{i}$.

colapsam aceleradamente até formar um primeiro horizonte durante esta era; depois o sistema se torna inevitavelmente dominado pelo vácuo, o que desacelera o processo de colapso formando um segundo horizonte (este segundo está associado ao estágio de Sitter final).

Finalmente, os sistemas dominados pelo fluido material durante o estágio intermediário $(\xi>1)$, mas com uma condição inicial muito diferente da condição para a formação de um horizonte aparente $\stackrel{\circ}{R}_{i}^{2}=\stackrel{\circ}{R}_{c i}^{2}\left(\mathrm{H}_{f} / \mathrm{H}_{i}, \xi\right)$ (o que retrasa a formação do primeiro horizonte) faz com que os dois horizontes do caso anterior sejam formado no mesmo instante, ou inclusive evitando a formação de qualquer horizonte (se $\stackrel{\circ}{R}_{i}^{2}<\stackrel{R}{R}_{c i}^{2}\left(\mathrm{H}_{f} / \mathrm{H}_{i}, \xi\right)$ ) sobre a superfície do núcleo estelar (ver a figura 10.4).

Nota-se que a condição para a formação de um horizonte aparente $\stackrel{\circ}{R}_{h}=-1$ é sempre atingida durante a era de Sitter final (quando $\stackrel{\circ}{R}^{2}$ decresce exponencialmente com o decorrer do tempo) para algum valor de $r_{d s}$ (radio de Sitter), algumas vesses inclusive para $r_{d s}>r_{\Sigma}$ (fora do núcleo estelar). Adicionalmente este horizonte de Sitter, associado com o estágio final desacelerado, faz com que $\stackrel{R}{R}>-1$ para rodo $r<r_{d s}$.

\subsubsection{Qual é o raio dos horizontes para $\mathrm{H}_{i} \ll \mathrm{H}_{f}$}

A equação (10.21), que é a condição geométrica para a formação de uma superfície esférica nula, pode ser reescrita como:

$$
\left(\frac{\mathrm{H}_{i}}{\mathrm{H}_{f}}\right)^{2}+\left\{1-\left(\frac{\mathrm{H}_{i}}{\mathrm{H}_{f}}\right)^{2}\right\}\left(\frac{R_{h}}{R_{i}}\right)^{2 \xi}=\left(\frac{R_{h}}{R_{i}}\right)^{2} \stackrel{\circ}{R}_{i}^{2}
$$

onde $\xi \in(1,3]$ para $\omega \in[0,1]$ e $v \in[0,1)$. A partir desta expressão podemos encontrar soluções aproximadas para o raio do primeiro e do segundo horizonte no caso em que eles existem. 
Horizonte externo: Para $\mathrm{H}_{i} \ll \mathrm{H}_{f}$ e $R_{h} \lesssim R_{i}$ a equação (10.31) podem ser aproximadas como

$$
\left(\frac{R_{h}}{R_{i}}\right)^{\xi-1}=\left|\stackrel{\circ}{R}_{i}\right|,
$$

é a condição para a formação de um horizonte aparente obtida previamente por [Campos and Lima, 2012]. Em nosso caso, a solução exata para o valor do raio do núcleo estelar quando o horizonte externo é formado se afasta da solução (10.32) por correções da ordem de $\left(\mathrm{H}_{i} / \mathrm{H}_{f}\right)^{2}$.

Horizonte interno: Quando $\mathrm{H}_{i} \ll \mathrm{H}_{f}$ mas $R_{h} \ll R_{i}$ a equação (10.31) pode ser aproximada por

$$
\left(\frac{\mathrm{H}_{i}}{\mathrm{H}_{f}}\right)^{2}=\left(\frac{R}{R_{i}}\right)^{2}\left\{\stackrel{\circ}{R}_{i}^{2}-\left(\frac{R}{R_{i}}\right)^{2 \xi-2}\right\},
$$

agora, se a condição inicial não é muito diferente da condição para a formação de um horizonte aparente $\left(\left|\AA_{i}\right|<1\right.$ mas não $\left.\ll 1\right)$ podemos desconsiderar o último termo na expressão acima, obtendo a seguinte valor

$$
R_{h}=\mathrm{H}_{f}^{-1}
$$

para o raio do horizonte de Sitter formado durante o estágio final do colapso. A solução exata difere da solução aproximada por correções da ordem de $\left(R_{h} / R_{i}\right)^{2 \xi-2} / \AA_{i}^{2}$ e da ordem $\left(\mathrm{H}_{i} / \mathrm{H}_{f}\right)^{2}$. Por outro lado, no limite de $\mathrm{H}_{f} \rightarrow \infty$ o raio do horizonte interno vai para zero tornando-se a singularidade do modelo [Campos and Lima, 2012], o que é formado no instante $t=t_{c} \equiv\left(\xi \mathrm{H}_{i}\right)^{-1}$ (ver equação (10.13)).

\subsubsection{Massa do sistema}

A função de massa invariante $m(t, r)$, em geral definida por (B.7) para sistemas esfericamente simétricos, e no caso da geometria FRW plana é descrita pela expressão (9.28) com $\kappa=0$ :

$$
m\left(t, r_{\Sigma}\right)=\frac{1}{2} R\left(1+g^{\mu v} R_{, \mu} R_{, v}\right)=\frac{1}{2} R \dot{R}^{2},
$$

é interpretada como a massa total do núcleo estelar no instante $t$, e em nosso caso pode se escrever como:

$$
m\left(t, r_{\Sigma}\right)=\frac{\mathrm{H}_{f}^{2}}{2} \frac{R^{3}}{1+\varrho_{1}\left(R / R_{i}\right)^{2 \xi}} .
$$

No instante de formação do horizonte externo (o que ocorre quando $\stackrel{\circ}{R}_{h}^{2}=1$ ) a função massa torna-se

$$
m\left(t_{h}, r_{\Sigma}\right)=R_{h} / 2
$$

onde $R_{h}$ é a maior das soluções da equação (10.21) (ver a figura 10.3).

Por outro lado, a massa do núcleo estelar durante a era de Sitter final (para um observador comóvel com a superfície do núcleo) apresente um decrescimento da forma:

$$
m_{d S}=\frac{1}{2} \mathrm{H}_{f}^{2} R_{\circledast}^{3} e^{3 \mathrm{H}_{I}\left(t-t_{\circledast}\right)} .
$$




\subsection{RESUMO DO CAPÍTULO}

No presente capítulo, estudamos o processo de colapso gravitacional de um núcleo estelar esfericamente simétrico, aproximadamente homogêneo e isotrópico nos estágios finais do colapso, e sendo composto por uma mistura de um fluido material e um vácuo cuja densidade cresce mais rapidamente que a densidade de energia material no regime de altas energias. Encontramos que existem dois comportamentos caracteristicamente diferentes referentes à formação ou não de horizontes aparentes envolta da superfície do núcleo estelar durante o estágio final do colapso, o que acontece sem a formação de singularidades.

Primeiro, para $\stackrel{\circ}{R}_{i}^{2} \ll 1$ (lembrando que $R\left(t_{i}\right)=R_{i}$ foi escolhido já dentro dos estágios intermediários do colapso, onde podemos assumir a homogeneidade e isotropia do núcleo estelar) e $\left\{\mathrm{H}_{f} / \mathrm{H}_{i}, \xi\right\}$ são tais que o colapso é inicialmente dominado pela densidade do vácuo; ou tais que o período de domínio do fluido material não é suficiente para atingir a condição de formação de um horizonte aparente dentro do núcleo antes que o sistema se torne dominado pelo vácuo, tendendo a uma densidade total constante durante a fase final desacelerada; nenhum horizonte é formado dentro do núcleo estelar para $R<R_{i}$. Em ambos os casos, podemos associar à era final de Sitter um horizonte, mas este possui um raio maior que o raio do núcleo estelar, portanto não é visto por nossas coordenadas internas, além de ser um horizonte aparente do tipo de Sitter.

Segundo, para $\stackrel{\circ}{R}_{i}^{2} \leqslant 1$ (próximo da condição para a formação de um horizonte aparente), $\xi>1 / 2$ (estágio intermediário dominado pelo fluido material) e $\mathrm{H}_{f} / \mathrm{H}_{i} \gg 1$ (estágio intermediário comprido) o sistema permanece dominado pelo fluido material (e portanto colapsando aceleradamente) durante um período de tempo suficiente até atingir a condição para a formação de um horizonte aparente sobre superfície do núcleo estelar. Mas à medida que o tempo passa, enquanto o raio do núcleo decresce, a velocidade de colapso cresce o suficiente e o sistema torna-se inevitavelmente dominado pelo vácuo. Devido a que o colapso se torna desacelerado indefinidamente, um horizonte associado à era de Sitter é formado. Como conclusão, o estado final não vai conter singularidades, mas vai estar coberto por dois horizontes.

Um terceiro caso é possível para valores intermediários dos parâmetros livres do modelo. Esta configuração retrasa a formação do horizonte externo até coincidir com a formação do horizonte interno.

Uma característica global do sistema é o estágio final dominado pela densidade do vácuo, levando ao núcleo estelar a um estágio assintoticamente estacionário e desacelerado, impedindo a formação de uma singularidade. No entanto, o sistema pode, dependendo das condições iniciais e do valor dos parâmetros livres, formar 2 horizontes, sendo o horizonte externo um horizonte atrapante externo futuro, que impede a um observador no infinito espacial tenha acesso ao estado final do processo de colapso, enquanto que o horizonte interno e um horizonte atrapante interno futuro, ver Hayward [1994].

Também se obteve que no limite $\mathrm{H}_{f} \rightarrow \infty$ o horizonte externo coincide com o horizonte de um buraco negro [Campos and Lima, 2012] enquanto que o horizonte interno se torna a singularidade do buraco, e os dois são atingidos em um intervalo de tempo finito (para um observador comóvel na superfície do núcleo estelar). 



\section{1}

\section{CONSIDERAÇÕES FINAIS}

Ao longo desta monografia discutimos diversas consequências físicas da presença de um termo $\Lambda$ variável tanto na evolução da história cósmica como no processo de colapso gravitacional.

Neste trabalho, a presença de uma densidade do vácuo dependente do tempo (ou equivalentemente um termo $\Lambda$ variável) se justifica pelos problemas da constante cosmológica e pelo chamado problema da coincidência, presentes no modelo padrão $\Lambda$ CDM. Como a escala temporal típica na evolução do universo FRW é o parâmetro de Hubble, é natural assumir que a densidade do vácuo deva ser uma função de $H$ e, portanto, decrescer ao longo da História cósmica. Nesse sentido, podemos também dizer que a densidade do vácuo atualmente é extremamente baixa porque o Universo é velho.

Neste trabalho mostramos que modelos de universo espacialmente planos contendo um fluido material e uma densidade do vácuo associada com um termo $\Lambda$ da forma $\Lambda(t)=c_{0}+c_{\alpha} H^{\alpha}$, possui um estado inicial não singular, acelerado e dominado pelo vácuo para todo $\alpha>2$. Durante o período inicial (fase de Sitter primordial), $\Lambda$ decresce lentamente, enquanto a densidade de energia do fluido material (radiação) cresce de forma rápida até dominar a evolução do universo, proporcionando assim uma saída natural do período inflacionário.

Posteriormente, durante os estágios desacelerados as duas densidades (radiação ou matéria e vácuo) decrescem de forma similar, acopladas pela equação de conservação do tensor momento energia total, o que alivia naturalmente o problema da coincidência cósmica.

Isto acontece até que a densidade do vácuo se aproxima da ordem de grandeza do termo constante $c_{0}$, quando termos do tipo $H^{\alpha}(\alpha>2)$ se tornam desprezíveis. A partir desse período o universo volta a ser dominado pelo vácuo, cuja densidade tende ao valor limite $c_{0}$, evoluindo para um estágio final do tipo de Sitter. Com isto, neste cenário, toda história cósmica ocorre entre 2 fases de Sitter extremas não singulares (no início e no final do Universo).

Neste tipo de modelo, a densidade inicial do vácuo $\Lambda$ pode possuir o valor estimado pela teoria de campos (da ordem da escala de energia da grande unificação). Subsequentemente, sua densidade decresce ao longo de toda a história cósmica até atingir o valor da densidade da energia escura observada hoje (da ordem da densidade crítica atual), dessa forma aliviando o problema da constante cosmológica.

Da análise da compatibilidade do modelo deflacionário com a segunda lei da termodinâmica, obtemos que os estágios inflacionário, de domínio da radiação e de domínio da matéria escura apresentam uma entropia total (associada ao horizonte aparente e a seu conteúdo material) que cresce sem limite, enquanto que o estágio final dominado pela energia escura remanescente conduz o modelo de Universo para um estado de entropia máxima (equilíbrio termodinâmico). 
Usando testes cosmológicos para baixos z's (comparação do modelo com observações recentes do parâmetro de Hubble em função do deslocamento para o vermelho, $z$, da função de crescimento das perturbações lineares, da posição do pico das oscilações acústicas dos bárions, e da luminosidade de supernovas tipo Ia) foi possível ajustar o parâmetro $v$ que diferencia entre o modelo deflacionário e o modelo padrão durante o universo recente, encontrando que $v=0.029 \pm 0.01 \mathrm{com} 68 \%$ de confiança $(v=0$ corresponde ao modelo padrão).

Numa outra linha de desenvolvimento, nossos estudos das consequências da presença de um vácuo dinâmico durante os últimos estágios do colapso gravitacional também revelaram alguns resultados interessantes.

Como foi visto, para um termo $\Lambda$ proporcional à densidade total de um núcleo estelar que colapsa gravitacionalmente, mostramos que a formação da singularidade não pode ser evitada. Esse resultado é independente da curvatura espacial do sistema na aproximação de homogeneidade e isotropia internas durante os estágios finais do colapso. No entanto, a geometria afeta as diferentes quantidades físicas. Por exemplo, quando comparados ao caso plano, um termo $\Lambda$ deste tipo (proporcional a densidade de energia total), produz um atraso (ou adiantamento) na formação do horizonte e da singularidade dependendo da geometria ser fechada (ou aberta). Além disso, existe também um correspondente decréscimo ou aumento da massa colapsada para ambos os casos.

$\mathrm{Na}$ análise do processo de colapso gravitacional de um núcleo estelar esfericamente simétrico, mostramos que na presença de uma densidade do vácuo com termos de ordem superior na taxa de contração do sistema, esta consegue dominar a evolução do colapso no limite de altas energias, e cresce assintoticamente até um valor constante impedindo a formação de um estado final singular. Encontramos também que este estágio final é assintoticamente do tipo de Sitter, e em coordenadas apropriadas pode ser reescrito numa forma estacionária.

Neste último casso, durante o processo de colapso podem ser formados 2, 1 ou mesmo nenhum horizonte aparente ao interior do núcleo colapsante. No caso em que 2 horizontes aparentes são formados, o horizonte externo é um horizonte atrapante externo futuro, sendo portanto equivalente, do ponto de vista de um observador externo, a um buraco negro.

Mostramos portanto que o modelo deflacionário analisado neste trabalho é consistente com as observações cosmológicas feitas em red-shift baixo (que vinculam o modelo tanto para o universo primordial quanto para o universo recente), tanto no nível de backgroud quando no nível de perturbações lineares. Adicionalmente este modelo conecta as duas eras aceleradas do universo, que são dominadas pelo vácuo dinâmico reduzindo o setor escuro+inflacionário, e evitando a singularidade inicial graças a que os estágios inicial e final do modelo são assimptoticamente de Sitter. Concluímos então que o modelo deflacionário constitui uma boa alternativa para o modelo cosmológico padrão, reproduzindo a história cósmica de forma natural e fornecendo a possibilidade de ser distinguível do modelo $\Lambda \mathrm{CDM}$ graças ao grande acúmulo de dados observacionais de alta precisão que estão sendo coletados atualmente. Como completeza, mostramos também que esta classe de modelos de vácuo dinâmico podem levar à formação de buracos negros não singulares, após o processo de colapso gravitacional, devido ao domínio do vácuo no regime de altas energias. 
Neste apêndice apresentaremos alguns conceitos importantes para o entendimento da geometria espaçotemporal, especialmente para a definição local de um buraco negro. Todos o conteúdo aqui apresentado é uma compilação de resultados de [Hawking and Ellis, 1973], [Hayward, 1994] e [Carroll, 2004].

\section{A.1 HIPER-SUPERFÍCIES}

Um mapa $\phi$ de uma variedade $\mathcal{M}$ de dimensão $n$ e $k$-vezes diferenciável, para uma variedade $\mathcal{M}^{\prime}$ de dimensão $n^{\prime}$ e $k^{\prime}$-vezes diferenciável, se diz um mapa $r$-vezes diferenciável $\left(r \leqslant k, r \leqslant k^{\prime}\right)$ se, para qualquer sistema de coordenadas locais em $\mathcal{M}$ e $\mathcal{M}^{\prime}$, as coordenadas do ponto imagem $\phi(p)$ em $\mathcal{M}^{\prime}$ são funções $r$-vezes diferenciáveis das coordenadas de $p$ em $\mathcal{M}$. Como o mapa não é em geral bijectivo, este pode não possuir uma inversa; e se possuir inversa ela não necessariamente vai ser $r$-vezes diferenciável.

Se $f$ é uma função em $\mathcal{M}^{\prime}$, o mapa $\phi$ define a função $\phi^{*} f$ em $\mathcal{M}$ como sendo a função cujo valor no ponto $p$ de $\mathcal{M}$ é igual ao valor de $f$ em $\phi(p)$, isto é:

$$
\phi^{*} f(p)=f(\phi(p))
$$

Assim, quando $\phi$ mapeia pontos de $\mathcal{M}$ em pontos de $\mathcal{M}^{\prime}, \phi^{*}$ mapeia funções linearmente de $\mathcal{M}^{\prime}$ em $\mathcal{M}$.

Se $\lambda(t)$ é uma curva que passa a traves do ponto $p$ em $\mathcal{M}$, então a curva imagem $\phi(\lambda(t))$ em $\mathcal{M}^{\prime}$ passa a través do ponto $\phi(p)$. Se $r \geqslant 1$, o vetor tangente a esta curva em $\phi(p)$ é denotado por $\left.\phi_{*}\left(\partial_{\lambda}\right)\right|_{p}$. Assim, $\phi_{*}$ é um mapa linear de $T_{p}(\mathcal{M})$ em $T_{\phi(p)}\left(\mathcal{M}^{\prime}\right)$, e pode ser caracterizado pela relação

$$
\left.X\left(\phi^{*} f\right)\right|_{p}=\left.\phi_{*} X(f)\right|_{\phi(p)}
$$

Usando o mapa vetorial $\phi_{*}$ de $\mathcal{M}$ para $\mathcal{M}^{\prime}$, se $r \geqslant 1$ pode-se definir um mapa linear de formas $\phi^{*}$ de $T_{\phi(p)}^{*}\left(\mathcal{M}^{\prime}\right)$ para $T_{p}^{*}(\mathcal{M})$ pela condição: a contração de vetores e 1-formas é preservada sobre os mapas gerados por $\phi$. Com isto, uma 1-forma $A \in T_{\phi(p)}^{*}$ é mapeada em uma 1-forma $\phi^{*} A \in T_{p}^{*}$ onde, para vetores arbitrários $X \in T_{p}$ se respeita a condição

$$
\left.\left\langle\phi^{*} A, X\right\rangle\right|_{p}=\left.\left\langle A, \phi_{*} X\right\rangle\right|_{\phi(p)}
$$

Um mapa $r$-vezes diferenciável, $r>0$, se diz um immersion se sua inversa é um mapa $r$-vezes diferenciável, ou seja, se por cada ponto $p \in \mathcal{M}$ há uma vizinhança $\mathscr{U}$ de $p$ em $\mathcal{M}$ tal que a inversa $\phi^{-1}$ restrita a $\phi(\mathscr{U})$ é também um mapa $r$-vezes diferenciável. Isto implica que $n \leqslant n^{\prime}$. Quando $r \geqslant 1, \phi$ 
é um immersion se e somente se este é injetiva em cada ponto $p \in \mathcal{M}$; neste caso $\phi_{*}$ é um isomorfismo de $T_{p}$ em $\phi_{*}\left(T_{p}\right) \subset T_{\phi(p)}$. Se diz então que a imagem $\phi(\mathcal{M})$ é uma immersed sub-variedade $n$-dimensional em $\mathcal{M}^{\prime}$. Esta sub-variedade pode em geral possuir auto-interseções, ou seja, o mapa $\phi$ pode não ser um mapa 1-1 globalmente mesmo quando ele seja 1-1 localmente. Um immersion é dito um imbedding se ele é um homomorfismo na sua imagem com a topologia induzida, ou seja, um imbbeding é um immersion 1-1.

Uma hiper-superfície $\Sigma$, com dimensão $(n-1)$, é uma immersed sub-variedade na variedade $(\mathcal{M}, g)$, com dimensão $n$, definida como todos os pontos $x^{\mu} \in \mathcal{M}$ tais que

$$
f(x)=\text { cte. }
$$

onde $f$ é uma função escalar definida sobre todo $\mathcal{M}$. Sobre a variedade $(\mathcal{M}, g)$ podemos definir o campo vetorial

$$
\zeta^{\mu}=g^{\mu \nu} \nabla_{\mu} f,
$$

que é perpendicular às hiper-superfícies $f=$ cte., como também é ortogonal a todos os vetores em $T_{p} \Sigma$ (o espaço de todos os vetores tangentes a $\Sigma$ ). Com isto, o campo vetorial definido como

$$
n^{\mu}=\frac{\zeta^{\mu}}{\zeta}
$$

onde $\zeta \equiv\left|\zeta^{\mu} \zeta_{\mu}\right|_{\Sigma}^{1 / 2}$, é um vetor normal às hiper-superfícies $f=c t e$., sempre que $\zeta^{\mu} \zeta_{\mu} \neq 0$. Se agora definimos as coordenadas $y^{i}=\left\{y^{1}, \cdots, y^{n-1}\right\}$ sobre a hiper-superfície $\Sigma$. No caso de hipersuperfícies nulas ver [Hawking and Ellis, 1973] e [Carroll, 2004]. O sistema de coordenadas $z, y^{i}$, tal que $z$ é o parâmetro afim da curva geodésica com vetor tangente $n^{\mu}$ no ponto $y^{i} \in \Sigma$, é um sistema coordenado para a variedade $\mathcal{M}$ conhecido como coordenadas normais Gaussianas. Com este sistema de coordenadas, o elemento de linha em $(\mathcal{M}, g)$ pode se escrever como:

$$
d s^{2}=\sigma d z^{2}+\gamma_{i j} d y^{i} d y^{j}
$$

onde $\sigma \equiv n_{\mu} n^{\mu}$, e $\gamma_{i j} \equiv g\left(\partial_{i}, \partial_{j}\right)$. Com isto temos que o operador:

$$
P_{\mu v} \equiv g_{\mu v}-\sigma n_{\mu} n_{v}
$$

projeta todos os vetores em $T_{p} \mathcal{M}$ em $T_{p} \Sigma$, serve como uma métrica induzida (pela métrica $g$ em $\mathcal{M}$ ) sobre a hiper-superfície $\Sigma$, e é conhecida como primeira forma fundamental da hiper-superfície.

Por completeza, a curvatura extrínseca da hiper-superfície $\Sigma($ immersed em $\mathcal{M})$ é dada por

$$
K_{\mu \nu} \equiv \frac{1}{2} \mathcal{L}_{n} P_{\mu \nu}=\frac{1}{2} \mathcal{L}_{n}\left(g_{\mu \nu}-\sigma n_{\mu} n_{v}\right)=\frac{1}{2} \mathcal{L}_{n} g_{\mu \nu}-\frac{\sigma}{2}\left(n_{\mu} \mathcal{L}_{n} n_{v}+n_{v} \mathcal{L}_{n} n_{\mu}\right)
$$

com

$$
\mathcal{L}_{n} n_{v}=\mathcal{L}\left(g_{v \alpha} n^{\alpha}\right)=n^{\alpha} \mathcal{L}_{n} g_{v \alpha}+g_{v \alpha} \mathcal{L}_{n} n^{\alpha}=n^{\alpha} \mathcal{L}_{n} g_{v \alpha}+g_{v \alpha}[n, n]^{\alpha}=n^{\alpha} \mathcal{L}_{n} g_{v \alpha}
$$

onde denotamos o comutador de dos campos vetoriais $X$ e $Y$ como $[X, Y]=X Y-Y X$. Se usamos agora $\mathcal{L}_{V} g_{\mu v}=\nabla_{\mu} V_{v}+\nabla_{v} V_{\mu}$ obtemos que

$$
\mathcal{L}_{n} n_{v}=n^{\alpha} \mathcal{L}_{n} g_{v \alpha}=\nabla_{\mu} n_{v}+\nabla_{v} n_{\mu}=\nabla_{v} \sigma+n^{\alpha} \nabla_{\alpha} n_{v}=n^{\alpha} \nabla_{\alpha} n_{v}
$$


com o que a curvatura extrínseca pode sempre se escrever como:

$$
K_{\mu v}=\nabla_{\mu} n_{v}+\nabla_{v} n_{\mu}-\sigma n_{\mu} n^{\alpha} \nabla_{\alpha} n_{v}-\sigma n_{v} n^{\alpha} \nabla_{\alpha} n_{\mu}=\nabla_{\mu} n_{v}-\sigma n_{\mu} n^{\alpha} \nabla_{\alpha} n_{v}
$$

conhecida também como segunda forma fundamental da hiper-superfície com vetor normal $n^{\alpha}$. No caso particular em que o vetor normal $n^{\alpha}$ é o vetor tangente de um feixe geodésico podemos usar a equação das geodésicas $n^{\alpha} \nabla_{\alpha} n_{v}=0$. Assim, a curvatura extrínseca de uma hiper-superfície com vetor normal $n^{\alpha}$ ( $n^{\alpha}$ tangente a um feixe geodésico) é:

$$
K_{\mu v}=\nabla_{\mu} n_{v}
$$

Em geral, a segunda forma fundamental pode ser escrita como [Santos, 1985; Eisenhart, 1949] ${ }^{1}$ :

$$
K_{\mu v}=-n_{\alpha}\left(x_{, \mu v}^{\alpha}-\Gamma_{\beta \gamma}^{\alpha} x_{, \mu}^{\beta} x_{, v}^{\gamma}\right)
$$

onde $x^{\mu}$ e $\Gamma_{\beta \gamma}^{\alpha}$ são as coordenadas e o simbolo de Christoffel da variedade $(\mathcal{M}, g)$.

\section{A.2 EStRUTURA CAUSAL DO ESPAÇO-TEMPO}

Devido a que as métricas Lorentzianas (com sinal -+++ ) não são definidas positivas, podemos classificar um vetor $V^{\mu}$ como sendo espacial, nulo ou temporal se $g_{\mu \nu} V^{\mu} V^{\nu}$ é maior, igual o menor do que zero. Similarmente, uma curva no espaço-tempo é dita espacial, nula ou temporal se seu vetor tangente é sempre espacial, nulo ou temporal, respetivamente. Por fim, uma superfície é dita espacial, nula ou temporal se seu vetor normal é do tipo temporal, nulo ou espacial, respetivamente.

Tomando um espaço-tempo orientável temporalmente, pode-se dividir os vetores não espaciais em cada ponto em dois grupos disjuntos: dirigidos para o futuro e dirigidos para o passado. Para conjuntos $\mathscr{S}$ e $\mathscr{U}$, o futuro cronológico $I^{+}(\mathscr{S}, \mathscr{U})$ de $\mathscr{S}$ relativo a $\mathscr{U}$ pode ser definido como o conjunto de todos os pontos em $\mathscr{U}$ que podem ser conectados a $\mathscr{S}$ por uma curva temporal dirigida para o futuro em $\mathscr{U}$, onde a curva deve ser de extensão finita, assim $I^{+}(\mathscr{S}, \mathscr{U})$ não contém $\mathscr{S} \cdot I^{+}(\mathscr{S}, \mathcal{M})$ será denotado por $I^{+}(\mathscr{S})$. Esta definição possui uma dual na que 'futuro' é substituído por 'passado' e $I^{+}$é substituído por $I^{-}$.

O futuro causal de $\mathscr{S}$ relativo a $\mathscr{U}$, é denotado por $J^{+}(\mathscr{S}, \mathscr{U})$, é definido como a união de $\mathscr{S} \cap \mathscr{U}$ com o conjunto de todos os pontos em $\mathscr{U}$ que podem ser conectados com $\mathscr{S}$ por médio de una curva não espacial dirigida para o futuro em $\mathscr{U}$. Como antes, $J^{+}(\mathscr{S}, \mathscr{U})$ é denotada simplesmente como $J^{+}(\mathscr{S})$. Esta é a região do espaço-tempo que pode ser causalmente afetada por eventos em $\mathscr{S}$. A definição de passado causal é análoga à definição anterior, substituindo 'futuro' por 'passado' e $J^{+}$por $J^{-}$.

O horismo futuro de $\mathscr{S}$ relativo a $\mathscr{U}$, denotado por $E^{+}(\mathscr{S}, \mathscr{U})$, é definido como $J^{+}(\mathscr{S}, \mathscr{U})-$ $I^{+}(\mathscr{S}, \mathscr{U})$. Denotamos $E^{+}(\mathscr{S}, \mathcal{M})$ como $E^{+}(\mathscr{S})$. Analogamente se define o horismo passado, substituindo 'futuro' por 'passado' e $E^{+}$por $E^{-}$.

\footnotetext{
${ }^{1}$ Equação (43.4) na referência [Eisenhart, 1949]
} 


\section{A.2.1 Superfícies de Cauchy}

Um conjunto $\mathscr{S}$ se diz ácrono se $I^{+}(\mathscr{S}) \cap \mathscr{S}$ é vazio, ou seja, se não há pontos em $\mathscr{S}$ que possam ser juntados por uma curva temporal. $\mathscr{S}$ se diz um conjunto futuro se $\mathscr{S} \supset I^{+}(\mathscr{S})$.

Proposição 1. Se $\mathscr{S}$ é um conjunto futuro, então a fronteira de $\mathscr{S}$, $\dot{S}$, é uma imbedded sub-variedade fechada e ácrona, e é chamada de fronteira ácrona.

A fronteira ácrona de um conjunto futuro $\mathscr{S}$ pode ser dividido em 4 subconjuntos disjuntos $\dot{\mathscr{S}}_{\mathrm{N}}, \dot{\mathscr{S}}_{+}$, $\dot{\mathscr{S}}_{-}$e $\dot{\mathscr{S}}_{0}$, tais que para um ponto $q \in \dot{\mathscr{S}}$ podem ou não existir pontos $p, r \in \dot{\mathscr{S}}$, onde $p \in E^{-}(q)-q$ e $r \in E^{+}(q)-q, \mathrm{e}$

$$
\begin{aligned}
& \text { se } \exists p, \quad \& \quad \exists r, \rightarrow q \in \dot{\mathscr{S}}_{N} \text {, } \\
& \text { se } \nexists p, \quad \& \quad \exists r, \rightarrow q \in \dot{\mathscr{S}}_{-} \text {, } \\
& \text { se } \exists p, \quad \& \quad \nexists r, \rightarrow q \in \dot{\mathscr{S}}_{+}, \\
& \text {se } \nexists p, \quad \& \quad \nexists r, \rightarrow q \in \dot{\mathscr{S}}_{0} \text {. }
\end{aligned}
$$

Se $q \in \dot{\mathscr{S}}_{N}$ então $r \in E^{+}(p)$ pela definição de $\dot{\mathscr{S}}_{N}$, mas $r \notin I^{+}(p)$, já que $\dot{\mathscr{S}}$ é um conjunto ácrono. Isto significa que existe um segmento geodésico nulo em $\dot{\mathscr{S}}$ que passa por $q$.

Se $q \in \dot{\mathscr{S}}_{+}$(respectivamente $\mathscr{S}_{-}$) então $q$ é o ponto final futuro (respectivamente passado) de uma geodésica nula em $\dot{\mathscr{S}}$.

Finalmente, o subconjunto $\dot{\mathscr{S}}_{0}$ é espacial, e define o que chamaremos de conjunto não causal.

O domínio de dependência futuro $D^{+}(\mathscr{S})$ de $\mathscr{S}$ é definido como o conjunto de todos os pontos $p \in \mathcal{M}$ tais que toda curva não espacial que passa por $p$, e que não possui um ponto final passado, também intersecta $\mathscr{S}$. A fronteira futura de $D^{+}(\mathscr{S})$, ou seja $D^{+}(\mathscr{S})-I^{-}\left(D^{+}(\mathscr{S})\right)$, marca o limite da região que pode ser predita a partir do conhecimento completo de $\mathscr{S}$. Dito conjunto fechado e ácrona é chamado horizonte de Cauchy de $\mathscr{S}$, e é denotado por $H^{+}(\mathscr{S})$. Se define $D(\mathscr{S})=D^{+}(\mathscr{S}) \cup D^{-}(\mathscr{S})$.

A borda de um conjunto ácrono $\mathscr{S}$ é conjunto de pontos $q \in \mathscr{S}$ tal que em toda vizinhança $\mathscr{U}$ de $q$ existem pontos $p \in I^{-}(q, \mathscr{U})$ e $r \in I^{+}(q, \mathscr{U})$ que podem ser juntados por uma curva temporal em $\mathscr{U}$ e que não intersecta $\mathscr{S}$. Portanto, se a borda de $\mathscr{S}$ é vazia para um conjunto ácrono não vazio $\mathscr{S}$, então $\mathscr{S}$ é uma imbedded sub-variedade 3-dimensional.

Proposição 2. Seja $\mathscr{S}$ um conjunto fechado ácrono. Então $H^{+}(\mathscr{S})$ é gerado por segmentos de geodésicas nulas que: não possuem pontos finais passados, ou possuem pontos finais passados na borda de $\mathscr{S}$.

Se diz que um conjunto não causal $\mathscr{S}$ sem borda é uma superfície parcial de Cauchy. Ou seja, é uma hiper-superfície espacial tal que não existem curvas não espaciais que intersectam a superfície mais que uma vez.

Um conjunto ácrono $\mathscr{S}$ tal que $E^{+}(\mathscr{S})$ ou $E^{-}(\mathscr{S})$ é compacto, é dito um conjunto atrapante. 


\section{A.2.2 Estrutura conforme da fronteira do espaço-tempo de Minkowsky}

No espaço-tempo de Minkowsky $\mathcal{M}=\left(\mathbb{R}^{4}, \eta\right)$, onde as componentes da métrica $\eta$ são $\eta_{\mu v}=\delta_{\mu v}(-1,1,1,1)$, podemos definir três tipos de fronteiras que determinam sua causalidade no infinito. Qualquer geodésica temporal dirigida para o futuro atinge $i^{+}\left(i^{-}\right)$para valores indefinidamente grandes e positivos (negativos) de seu parâmetro afim, assim pode-se considerar qualquer geodésica temporal como tendo sua origem em $i^{-}$e seu fim em $i^{+}$. Similarmente pode-se considerar toda geodésica nula como tendo sua origem em $\mathscr{I}^{-}$e seu fim em $\mathscr{I}^{+}$, enquanto que geodésicas espaciais nascem e morrem em $i^{0}$. Assim, pode-se considerar $i^{+}\left(i^{-}\right)$como representando o infinito temporal futuro (passado) de $\mathcal{M}$, $\mathscr{I}^{+}\left(\mathscr{I}^{-}\right)$como representando o infinito nulo futuro (passado) de $\mathcal{M}$, e $i^{0}$ como representando o infinito espacial de $\mathcal{M}$.

\section{A.2.3 Outros Horizontes}

Se considerarmos uma família de partículas, cujas histórias são geodésicas temporais, a existência de um horizonte de partículas pode ser pensado como sendo uma consequência de que o infinito nulo passado $\mathscr{I}^{-}$seja uma hiper-superfície espacial, de forma tal que se $\mathscr{I}^{-}$for nulo então não haverá horizonte de partículas para nenhum observador geodésico. Mas quando $\mathscr{I}^{-}$não for espacial, o horizonte de partículas ainda pode ser não vazio para trajetórias não geodésicas, tais como partículas com aceleração própria constante, ou seja, o horizonte de partículas depende fortemente do observador. No caso em que tanto $\mathscr{I}^{-}$como $\mathscr{I}^{+}$são espaciais, todas as geodésicas temporais tem origem em $\mathscr{I}^{-}$e acabam em $\mathscr{I}^{+}$. Seja $p$ algum evento sobre a linha de mundo de uma partícula $\mathscr{O}$. O cone de luz passado de $p$ é o conjunto de eventos, no espaço-tempo, que podem ser observados por $\mathscr{O}$ naquele instante. Algumas linhas de mundo de outras partículas podem atravessar o cone de luz de $p$, ditas partículas são visíveis por $\mathscr{O}$. No entanto, também existem partículas tais que suas linhas de mundo não possuem interseção com o cone de luz de $p$, ditas partículas ainda não foram vistas por $\mathscr{O}$ até o instante em que ocorre o evento $p$. Passado um tempo, $\mathscr{O}$ pode observar mais partículas, mas sempre vão existir partículas que ainda não foram observadas por $\mathscr{O}$ naquele novo instante. Com isto, podem-se dividir as partículas entre as que já foram vistas por $\mathscr{O}$ em $p$ e aquelas que ainda não foram vistas em $p$. O horizonte de partículas de $\mathscr{O}$ em $p$ representa a historia completa (as linhas de mundo passada e futura) das partículas que estão no limite da visão de $\mathscr{O}$ no instante em que ocorre o evento $p$, e é claramente dependente do observador. Observadores comoveis em um espaço-tempo com um infinito nulo passado $\mathscr{I}^{-}$temporal não possuem horizonte de partículas.

Todos os eventos fora do cone de luz passado de $p$ são eventos que nunca foram observados por $\mathscr{O}$ até o instante em que ocorre o evento $p$. No caso em que infinito nulo passado $\mathscr{I}^{-}$é espacial, a linha de mundo de $\mathscr{O}$ acaba em um ponto $q$ em $\mathscr{I}^{-}$. O cone de luz passado de $q$ é uma fronteira entre os eventos que para algum tempo serão observados, ou foram observados, por $\mathscr{O}$ e entre os eventos que nunca serão observados por $\mathscr{O}$. Dita superfície é chamada de horizonte futuro de eventos da linha de mundo de $\mathscr{O}$, e claramente depende de $\mathscr{O}$. Para um observador geodésico $\mathscr{O}$, a existência de um horizonte futuro de eventos pode ser pensado como a consequência de que infinito nulo futuro $\mathscr{I}^{+}$seja uma hiper-superfície espacial, de forma tal que se $\mathscr{I}^{+}$é nulo não haverá horizonte futuro de eventos para observadores geodésicos. 


\section{A.2.4 Completeza geodésica}

Dada uma métrica $r$-vezes diferenciável, para um ponto $p$ de $\mathcal{M}$ e um vetor $X_{p}$ em $p$, existe uma geodésica maximal $\lambda(v)$ em $\mathcal{M}$ com ponto inicial $p$ e direção inicial $X_{p}$, ou seja, $\lambda(v=0)=p$ e $\left.\left(\partial_{v}\right) \lambda\right|_{v=0}=X_{p}$, onde $v$ é o parâmetro afim da equação geodésica. Se $r \geqslant 2$ a geodésica é única e depende diferencialmente de $p$ e $X_{p}$. Assim, se $r \geqslant 2$ pode se definir o mapa $(r-1)$-vezes diferenciável $\exp : T_{p} \rightarrow \mathcal{M}$, onde para cada $X \in T_{p}, \exp (X)=\lambda(1)$ é um ponto em $\mathcal{M}$. Este mapa pode não estar definido para todo $X \in T_{p}$, já que a geodésica $\lambda(t)$ pode não estar definida para todo $v$. Se $v$ pode tomar todos os valores, a geodésica $\lambda(v)$ será dita uma geodésica completa. Dizemos que uma variedade $\mathcal{M}$ é uma variedade geodesicamente completa se toda geodésica em $\mathcal{M}$ é completa, isto é, se exp esta definido em todo $T_{p}$ para todo $p$ em $\mathcal{M}$.

Existe uma vizinhança aberta $\mathcal{N}_{0}$ na origem de $T_{p}$ e uma vizinhança aberta $\mathcal{N}_{p}$ de $p$ em $\mathcal{M}$ tal que o mapa exp é uma difeomorfismo $r$-vezes diferenciável de $\mathcal{N}_{0}$ em $\mathcal{N}_{p}$. Tal vizinhança $\mathcal{N}_{p}$ é dita vizinhança normal de $p$. Se adicionalmente, todo ponto $q$ de $\mathcal{N}_{p}$ pode ser juntado com qualquer outro ponto $s$ em $\mathcal{N}_{p}$ por uma única geodésica totalmente contida em $\mathcal{N}_{p}$, a vizinhança $\mathcal{N}_{p}$ se diz uma vizinhança normal convexa.

Uma curva contínua $\gamma: F \rightarrow \mathcal{M}$, onde $F$ é um intervalo conexo de $\mathbb{R}$, é uma curva não espacial e dirigida para o futuro se para todo $t \in F$ há uma vizinhança $G$ de $t$ em $F$ e uma vizinhança normal convexa $\mathscr{U}$ de $\gamma(t)$ em $\mathcal{M}$ tal que para qualquer $t_{1} \in G, \gamma\left(t_{1}\right) \in J^{-}(\gamma(t), \mathscr{U})-\gamma(t)$ se $t_{1}<t$, e $\gamma\left(t_{1}\right) \in J^{+}(\gamma(t), \mathscr{U})-\gamma(t)$ se $t_{1}>t$. Uma curva $\gamma$ temporal dirigida para o futuro é definida de forma similar se substituindo $J$ por $I$.

Um ponto $p$ se diz um ponto final futuro de uma curva não espacial dirigida para o futuro $\gamma: F \rightarrow \mathcal{M}$ se para toda vizinhança $\mathscr{V}$ de $p$ há um $t \in F$ tal que $\gamma\left(t_{1}\right) \in \mathscr{V}$ para todo $t_{1} \in F$ com $t_{1} \geqslant t$. Analogamente se define o ponto limite de uma curva não espacial dirigida para o passado.

Pode ser mostrado [Hawking and Ellis, 1973] que para uma vizinhança coordenada normal convexa $\mathscr{U}$ de $q$, todos os pontos que podem ser juntados a q por médio de uma curva temporal (respectivamente, não espacial) em $\mathscr{U}$ são da forma $\exp _{q}(X)$, onde $X \in T_{q}$ e $g(X, X)<0$ (respectivamente, $\left.\leqslant 0\right)$. Ou seja, as geodésias nulas de $q$ formam a fronteira da região que pode ser conectada a $q$ mediante uma geodésica temporal ou não espacial dentro de $\mathscr{U}$. Assim, em $\mathscr{U}$, a fronteira de $I^{+}(q, \mathscr{U})$ ou $J^{+}(q, \mathscr{U})$ esta formada pelas geodésicas nulas de $q$ dirigidas para o futuro.

\section{A.2.5 Geodésicas temporais}

Uma 3-superfície espacial $\mathscr{H}$ é uma sub-variedade 3-dimensional imbedded em $(\mathcal{M}, g)$, definida localmente por $f=0$ onde $f$ é uma função duas vezes diferenciável e $g^{\alpha \beta} f_{; \alpha} f_{; \beta}<0$ quando $f=0$. Definindo $\mathscr{N}$, o vetor normal unitário a $\mathscr{H}$, como $\mathcal{N}^{\gamma}=\left(-g^{\alpha \beta} f_{; \alpha} f_{; \beta}\right)^{-1 / 2} g^{\gamma \sigma} f_{; \sigma}$ e a segunda forma fundamental $K$ de $\mathscr{H}$ como $K_{\alpha \beta}=P_{\alpha}^{\gamma} P_{\beta}^{\delta} \mathscr{N}_{\gamma ; \delta}$, onde $P_{\alpha \beta}=g_{\alpha \beta}+\mathscr{N}_{\alpha} \mathscr{N}_{\beta}$ é a primeira forma fundamental de $\mathscr{M}$. A congruência de geodésicas temporais ortogonais a $\mathscr{M}$ esta formada pelas geodésicas com vetor unitário tangente $V$ igual ao vetor unitário $\mathscr{N}$ normal a $\mathscr{H}$. Com isto $V_{\alpha ; \beta}=K_{\alpha \beta}$ em $\mathscr{H}$ é simétrico. 
$\mathrm{O}$ vetor $Z$ que representa a separação entre uma geodésica normal a $\mathscr{H}$ e a geodésica $\gamma(s)$ normal a $\mathscr{H}$ obedece a equação de Jacobi:

$$
\frac{d^{2}}{d s^{2}} Z^{\alpha}=-R_{a 4 b 4} Z^{\beta}, \quad(a, b=1,2,3),
$$

e em um ponto $q$ em $\gamma(s)$ em $\mathscr{H}$ obedece a condição inicial:

$$
\frac{d}{d s} Z^{a}=\left.K_{a b} Z^{\beta}\right|_{q}
$$

O campo de Jacobi ao longo de $\gamma(s)$ pode ser reescrito como:

$$
Z^{a}(s)=\left.A_{a b}(s) Z^{b}\right|_{q}
$$

onde

$$
\frac{d^{2}}{d s^{2}} A_{a b}=-R_{a 4 c 4} A_{c b}
$$

e em $q, A_{a b}$ é a matriz unidade e

$$
\frac{d}{d s} A_{a b}=K_{a c} A_{c b} .
$$

Se diz que o ponto $p$ em $\gamma(s)$ é conjugado a $\mathscr{H}$ ao longo de $\gamma(s)$ se existe um campo de Jacobi ao longo de $\gamma(s)$, não identicamente nulo, tal que satisfaz a condição inicial (A.17) em $q$ é se anula em $p$. Ou seja, $p$ é conjugado a $\mathscr{H}$ ao longo de $\gamma(s)$ se e somente se $A_{a b}$ é singular em $q$.

Proposição 3. Se $R_{\alpha \beta} V^{\alpha} V^{\beta} \geqslant 0$ e $K_{\alpha \beta} g^{\alpha \beta}<0$, existe um ponto conjugado a $\mathscr{H}$ ao longo de $\gamma(s)$ a uma distância menor que $3 /\left(-K_{\alpha \beta} g^{\alpha \beta}\right)$ de $\mathscr{H}$.

\section{A.2.6 Geodésicas nulas}

A solução à equação

$$
\frac{d^{2}}{d v^{2}} Z^{m n}=-R_{m 4 n 4} Z^{n}, \quad(m, n=1,2)
$$

ao longo de uma geodésica nula $\gamma(v)$, é chamada de campo de Jacobi ao longo $\gamma(v)$. Se diz que $p$ é conjugado a $q$ ao longo da geodésica nula $\gamma(v)$ se existe um campo de Jacobi ao longo de $\gamma(v)$, não identicamente nulo, tal que se anula em $p$ e em $q$. O ponto $p$ pode ser interpretado como o ponto onde geodésicas infinitesimalmente próximas, que passam por $q$, se intersectam. O campo de Jacobi ao longo de $\gamma(v)$, que é nulo em $q$, pode ser representado pela matriz $2 \times 2 \hat{A}_{m n}$ :

$$
Z^{m}(v)=\left.\hat{A}_{m n} \frac{d}{d v} Z^{n}\right|_{q}
$$

Uma 2-superfície espacial $\mathscr{S}$ como uma sub-variedade 2-dimensional imbedded, em $(\mathcal{M}, g)$, é definida localmente por $f_{1}=0, f_{2}=0$ onde $f_{1}$ e $f_{2}$ são funções duas vezes diferenciáveis tais que quando $f_{1}=0$, $f_{2}=0$ então $f_{1 ; \alpha}$ e $f_{2 ; \beta}$ são diferentes de zero, são não paralelas, e

$$
\left(f_{1 ; \alpha}+\mu f_{2 ; \alpha}\right)\left(f_{1 ; \beta}+\mu f_{2 ; \beta}\right) g^{\alpha \beta}=0
$$


para dois valores reais e diferentes $\mu_{1}$ e $\mu_{2}$ de $\mu$. Assim, todo vetor na 2-superfície $\mathscr{S}$ é necessariamente espacial. Definindo $\mathscr{N}_{1}^{\alpha}$ e $\mathscr{N}_{2}^{\alpha}$, os vetores nulos normais a $\mathscr{S}$, como proporcionais a $g^{\alpha \beta}\left(f_{1 ; \beta}+\mu_{1} f_{2 ; \beta}\right)$ e $g^{\alpha \beta}\left(f_{1 ; \beta}+\mu_{2} f_{2 ; \beta}\right)$, respectivamente, e normalizados da forma

$$
\mathscr{N}_{1}^{\alpha} \mathscr{N}_{2}^{\beta} g_{\alpha \beta}=-1
$$

e introduzindo dois vetores espaciais unitários $Y_{1}^{\alpha}$ e $Y_{2}^{\alpha}$ ortogonais a cada um dos vetores $\mathscr{N}_{1}$ e $\mathscr{N}_{2}$, pode-se completar uma base pseudo-ortonormal para $T_{p}$ em $\mathscr{S}$. Podem se definir os dois tensores nulos da segunda forma fundamental em $\mathscr{S}$ como

$$
{ }_{n} K_{\alpha \beta}=-\mathscr{N}_{n \lambda ; \delta}\left(Y_{1}^{\lambda} Y_{1 \alpha}+Y_{2}^{\lambda} Y_{2 \alpha}\right)\left(Y_{1}^{\delta} Y_{1 \beta}+Y_{2}^{\delta} Y_{2 \beta}\right), \quad(n=1,2)
$$

e são anti-simétricos.

Existem duas famílias de geodésicas nulas normais à 2-superfície espacial $\mathscr{S}$, correspondentes às duas direções normais $\mathscr{N}_{1}$ e $\mathscr{N}_{2}$. Considerando unicamente a segunda família, é possível fixar a base pseudoortogonal como $E_{1}=Y_{1}, E_{2}=Y_{2}, E_{3}=\mathscr{N}_{1}$ e $E_{4}=\mathscr{N}_{4}$ em $\mathscr{S}$ e propagá-la ao longo das geodésicas nulas. A projeção do vetor $Z$, que representa a separação entre a geodésica nula $\gamma(v)$ (com vetor tangente $V$ ) e uma geodésica nula vizinha, na direção paralela a $\gamma(v)$ satisfaz a equação

$$
\frac{d}{d v} Z^{m}=V_{; n}^{m} Z^{n}
$$

com a condição inicial

$$
\frac{d}{d v} Z^{m}={ }_{2} K_{m n} Z^{n}
$$

em $q$ sobre $\gamma(v)$ em $\mathscr{S}$.

Proposição 4. Se $R_{\alpha \beta} V^{\alpha} V^{\beta} \geqslant 0$ sobre toda a variedade $(\mathcal{M}, g)$ e ${ }_{2} K_{\alpha \beta} g^{\alpha \beta}$ é negativo, existe um ponto conjugado a $\mathscr{S}$ ao longo de $\gamma(v)$ a uma distancia menor que $2 /\left(-{ }_{2} K_{\alpha \beta} g^{\alpha \beta}\right)$ de $\mathscr{S}$.

Uma superfície fechada atrapante $\mathscr{T}$ é uma superfície espacial fechada (ou seja, compacta e sem fronteira) 2-dimensional tal que duas famílias de geodésicas nulas ortogonais a $\mathscr{T}$ convergem a $\mathscr{T}$, isto é, se ${ }_{1} K_{\alpha \beta} g^{\alpha \beta}$ e ${ }_{2} K_{\alpha \beta} g^{\alpha \beta}$ são negativos, onde ${ }_{1} K_{\alpha \beta}$ e ${ }_{2} K_{\alpha \beta}$ são os dois tensores nulos da segunda forma fundamental de $\mathscr{T}$.

\section{A.2.7 Buracos negros em espaços assintoticamente simples}

Se diz que a condição de condição forte de causalidade é respeitada no ponto $p$ se toda vizinhança de $p$ contem uma vizinhança de $p$ tal que nenhuma curva não espacial intersecta ela mais que uma vez.

Um espaço $(\mathcal{M}, g)$ orientável espacialmente e temporalmente se diz um espaço assintoticamente simples se existe um espaço $(\widetilde{\mathcal{M}}, \widetilde{g})$ fortemente causal e um imbedding $\theta: \mathcal{M} \rightarrow \tilde{\mathcal{M}}$ que mapeia $\mathcal{M}$ como uma variedade com fronteira suave $\partial \mathcal{M}$ em $\widetilde{\mathcal{M}}$, tal que:

1. há uma função suave $\Omega$ em $\widetilde{\mathcal{M}}$ tal que em $\theta(\mathcal{M}), \Omega$ é positiva e $\Omega^{2} g=\theta_{*}(\widetilde{g})$; o que significa que $\widetilde{g}$ é conforme a $g$ em $\theta(\mathcal{M})$

2. em $\partial \mathcal{M}, \Omega=0$ e $d \Omega \neq 0$ 
3. toda geodésica nula em $\mathcal{M}$ possui dois pontos finais em $\partial \mathcal{M}$

$(\mathcal{M}, g)$ é um espaço assintoticamente simples e vazio se é assintoticamente simples e $R_{\alpha \beta}=0$ para uma vizinhança aberta de $\partial \mathcal{M}$ em $\overline{\mathcal{M}} \equiv \mathcal{M} \cup \partial \mathcal{M}$. A fronteira $\partial \mathcal{M}$ pode ser interpretada como estando no infinito, no sentido em que o parâmetro afim na métrica $g$ para uma geodésica nula em $\mathcal{M}$ cresce sem limite perto de $\partial \mathcal{M}$. Isto é devido a que o parâmetro afim $v$ na métrica $g$ é relacionado com parâmetro afim na métrica $\tilde{g}$ por $d v / d \tilde{v}=\Omega^{-2}$, e já que $\Omega=0$ em $\partial \mathcal{M}$, teremos que $\int d v$ diverge. Adicionalmente, pela condição 2 e dado que $R_{\alpha \beta}=0$ pode se mostrar que $\partial \mathcal{M}$ é uma hiper-superfície nula. Devido a que $\partial \mathcal{M}$ é uma hiper-superfície nula, $\mathcal{M}$ esta contido localmente no passado ou futuro de $\partial \mathcal{M}$, o que mostra que $\partial \mathcal{M}$ deve estar formado por duas componentes não conexas $\mathscr{I}^{+}$e $\mathscr{I}^{-}$.

Proposição 5. Em um espaço $(\mathcal{M}, g)$ assintoticamente simples e vazio, $\mathscr{I}^{+}$e $\mathscr{I}^{-}$são topologicamente $\mathbb{R}^{1} \times S^{2}$, e $\mathcal{M}$ é topologicamente $\mathbb{R}^{4}$.

Se $(\mathcal{M}, g)$ possui uma região assintoticamente simples e vazia, existe um espaço $(\widetilde{\mathcal{M}}, \widetilde{g})$ em que $(\mathcal{M}, g)$ é conformalmente imbedded como uma variedade com fronteira $\overline{\mathcal{M}}=\mathcal{M} \cup \partial \mathcal{M}$, onde a fronteira $\partial \mathcal{M}$ de $\mathcal{M}$ em $\widetilde{\mathcal{M}}$ consiste de duas superfícies nulas $\mathscr{I}^{+}$e $\mathscr{I}^{-}$que representam os infinitos nulos futuro e passado respectivamente. Seja $\mathscr{S}$ uma superfície parcial de Cauchy em $\mathcal{M}$. Se diz que um espaço $(\mathcal{M}, g)$ é assintoticamente previsível a partir de $\mathscr{S}$ se $\mathscr{I}^{+}$esta contido na clausura de $D^{+}(\mathscr{S})$ na variedade conforme $\widetilde{\mathcal{M}}$.

Proposição 6. Se $(\mathcal{M}, g)$ é assintoticamente previsivel a partir de uma superfície parcial de Cauchy $\mathscr{S}$, e se $R_{\alpha \beta} V^{\alpha} V^{\beta} \geqslant 0$ para todo vetor nulo $V^{\alpha}$, então uma superfície fechada atrapante $\mathscr{T}$ em $D^{+}(\mathscr{S})$ não pode intersectar $J^{-}\left(\mathscr{I}^{+}, \overline{\mathcal{M}}\right)$.

Ou seja, uma superfície fechada atrapante em $D^{+}(\mathscr{S})$, em um espaço que seja assintoticamente predizível no futuro, debe estar contida em $\mathcal{M}-J^{-}\left(\mathscr{I}^{+}, \overline{\mathcal{M}}\right)$. Portanto, deveria ter um horizonte de eventos futuros não trivial $\dot{J}^{-}\left(\mathscr{I}^{+}, \overline{\mathcal{M}}\right)$, que é uma fronteira ácrona gerada por segmentos de geodésicas nulas que podem ter pontos finais passados mas não pontos finais futuros (ver A.2.4).

Lema 1. Se $(\mathcal{M}, g)$ é assintoticamente previsivel a partir de uma superfície parcial de Cauchy $\mathscr{S}$, se $R_{\alpha \beta} V^{\alpha} V^{\beta} \geqslant 0$ para todo vetor nulo $V^{\alpha}$, e se existe um horizonte de eventos não vazio $\dot{J}^{-}\left(\mathscr{I}^{+}, \overline{\mathcal{M}}\right)$, então a expansão $\hat{\theta}={ }_{2} K_{\alpha \beta} g^{\alpha \beta}$ das geodésicas nulas geradoras de $\dot{J}^{-}\left(\mathscr{I}^{+}, \overline{\mathcal{M}}\right)$ é não negativa em $\dot{J}^{-}\left(\mathscr{I}^{+}, \overline{\mathcal{M}}\right) \cap$ $D^{+}(\mathscr{S})$.

Em um espaço com futuro assintoticamente previsível, $J^{+}(\mathscr{S}) \cap J^{-}\left(\mathscr{I}^{-}, \overline{\mathcal{M}}\right)$ esta contido em $D^{+}(\mathscr{S})$. Se existe um ponto $p$ sobre o horizonte de eventos em $J^{+}(\mathscr{S})$, que não esta em $D^{+}(\mathscr{S})$, uma perturbação poderia conduzir a que $p \in J^{-}\left(\mathscr{I}^{+} \overline{\mathcal{M}}\right)$, e portanto seria visível pelo infinito, o que significaria que o espaço não seria mais assintoticamente previsível. Este fato obriga fazer uma extensão da definição de um futuro assintoticamente previsível: se diz que um espaço é fortemente assintoticamente previsível, a partir de uma superfície parcial de Cauchy $\mathscr{S}$, se $\mathscr{I}^{+}$esta contido na clausura de $D^{+}(\mathscr{S})$ em $\overline{\mathcal{M}}$, e $J^{+}\left(\mathscr{S} \cap \overline{J^{-}}\left(\mathscr{I}^{+}, \overline{\mathcal{M}}\right)\right)$ esta também contido em $D^{+}(\mathscr{S})$.

Proposição 7. Se $(\mathcal{M}, g)$ é fortemente assintoticamente previsível a partir de uma superfície parcial de Cauchy $\mathscr{S}$, existe um homomorfismo $\alpha:(0, \infty) \times \mathscr{S} \rightarrow D^{+}(\mathscr{S})-\mathscr{S}$, tal que para cada $\tau \in(0, \infty)$, $\mathscr{S}(\tau) \equiv(\{\tau\} \times \mathscr{S})$ é uma superfície parcial de Cauchy tal que: 
1. $\operatorname{para} \tau_{2}>\tau_{1}, \mathscr{S}\left(\tau_{2}\right) \subset I^{+}\left(\mathscr{S}\left(\tau_{1}\right)\right)$;

2. para cada $\tau$, a borda de $\mathscr{S}(\tau)$ na variedade conforme $\widetilde{\mathcal{M}}$ é uma 2-superfície espacial $\mathscr{Q}(\tau)$ em $\mathscr{I}^{+}$tal que para $\tau_{2}>\tau_{1}, \mathscr{Q}\left(\tau_{2}\right)$ esta estritamente no futuro de $\mathscr{Q}\left(\tau_{1}\right)$;

3. para cada $\tau, \mathscr{S}(\tau) \cup\left\{\mathscr{I}^{+} \cap J^{-}(\mathscr{Q}(\tau), \overline{\mathcal{M}})\right\}$ é uma superfície de Cauchy em $\overline{\mathcal{M}}$ para $D(\mathscr{S})$.

Em outras palavras, $\mathscr{S}(\tau)$ é uma família de superfícies espaciais homeomorfas a $\mathscr{S}$ tais que cobrem $D^{+}(\mathscr{S})-\mathscr{S}$ e intersectam $\mathscr{I}^{+}$.

Segue como resultado que se existe um horizonte de eventos $\dot{J}^{-}\left(\mathscr{I}^{+}, \overline{\mathcal{M}}\right)$ em $D^{+}(\mathscr{S})$ em um espaço assintoticamente previsível no futuro, para $\tau$ grande o suficiente, $\mathscr{S}(\tau)$ intersecta o horizonte. Se define um buraco negro na superfície $\mathscr{S}(\tau)$ como sendo uma componente conexa do conjunto $\mathscr{B}(\tau) \equiv \mathscr{S}(\tau)-J^{-}\left(\mathscr{I}^{+}, \overline{\mathcal{M}}\right)$; ou seja, este é a região de $\mathscr{S}(\tau)$ da que fótons ou partículas não poder espapar para $\mathscr{I}^{+}$.

Proposição 8. Seja $(\mathcal{M}, g)$ um espaço fortemente assintoticamente previsivel a partir de uma superfície parcial de Cauchy $\mathscr{S}$ que satisfaz $(\alpha)$ e $(\beta)$, então

1. a superfície $\mathscr{S}(\tau)$ também satisfaz $(\alpha)$ e $(\beta)$;

2. para cada $\tau, \partial \mathscr{B}_{1}(\tau)$, a fronteira de um buraco negro $\mathscr{B}_{1}(\tau)$ em $\mathscr{S}(\tau)$, é compacto e conexo.

Se diz que $(\mathcal{M}, g)$ é um espaço predizível regular se é fortemente assintoticamente predizível no futuro a partir de uma superfície parcial de Cauchy $\mathscr{S}$ e se satisfaz as propriedades $(\alpha),(\beta)$ e $(\gamma)$ :

$(\alpha) \mathscr{S} \cap \overline{J^{-}}\left(\mathscr{I}^{+}, \overline{\mathcal{M}}\right)$ é homeomorfo a $\mathbb{R}^{3}-\{$ um aberto com clausura compacta $\}$

( $\beta) \mathscr{S}$ é simplesmente conexo

$(\gamma)$ para $\tau$ suficientemente grande, $\mathscr{S}(\tau) \cap \overline{J^{-}}\left(\mathscr{I}^{-}, \overline{\mathcal{M}}\right)$ esta contido em $\bar{J}^{+}\left(\mathscr{I}^{-}, \overline{\mathcal{M}}\right)$.

A definição apresentada até agora, de um buraco negro, em função do horizonte de eventos $\dot{J}^{-}\left(\mathscr{I}^{+}, \overline{\mathcal{M}}\right)$, mas dada uma superfície parcial de Cauchy $\mathscr{S}(\tau)$, não é possível encontrar a posição do horizonte de eventos sem encontrar antes a evolução de todo o domínio de dependência futuro da superfície. Para efeitos práticos, é mais conveniente definir uma diferente classe de horizonte que dependa somente das propriedades do espaço-tempo na superfície $\mathscr{S}(\tau)$.

A proposição 6 assegura que uma superfície fechada atrapante em $\mathscr{S}(\tau)$, em um espaço predizível regular a partir de uma superfície parcial de Cauchy $\mathscr{S}$, esta contido em $\mathscr{B}(\tau)$. Este resultado depende apenas do fato de que as geodésicas nulas ortogonais à 2-superfície, e dirigidas para fora desta, convirjam ou não. Se diz que uma 2-superfície espacial orientável e compacta em $D^{+}(\mathscr{S})$ é um superfície atrapante exterior se a expansão $\hat{\theta}$ das geodésicas nulas ortogonais à superfície e dirigidas para fora é não positiva. Definimos uma região atrapante $\mathscr{T}(\tau)$ na superfície $\mathscr{S}(\tau)$ como o conjunto de todos os pontos $q \in \mathscr{S}(\tau)$ tais que existe uma superfície atrapante exterior $\mathscr{P}$ em $\mathscr{S}(\tau)$ que interseca o ponto $q$.

Proposição 9. Seja $(\mathcal{M}, g)$ um espaço predizivel regular a partir de uma superfície parcial de Cauchy $\mathscr{S}$, no que $R_{\alpha \beta} K^{\alpha} K^{\beta} \geqslant 0$ para todo vetor nulo $K^{\alpha}$. Então, uma superfície atrapante exterior $\mathscr{P}$ em $D^{+}(\mathscr{S})$ não intersecta $J^{-}\left(\mathscr{I}^{+}, \overline{\mathcal{M}}\right)$. 
Dizemos que a fronteira externa $\partial \mathscr{T}_{1}(\tau)$ de uma componente conexa $\mathscr{T}_{1}(\tau)$ de uma região atrapante $\mathscr{T}(\tau)$, é um horizonte aparente. Portanto, a existência de um horizonte aparente $\partial \mathscr{T}_{1}(\tau)$ implica a existência de uma componente $\partial \mathscr{S}_{1}(\tau)$ de um horizonte de eventos no seu exterior, ou coincidindo com este (o inverso não é necessariamente verdade).

Proposição 10. Cada componente $\partial \mathscr{T}(\tau)$ é uma 2-superfície tal que las geodésicas ortogonais a esta e dirigidas para fora possuem convergência $\hat{\theta}$ nula em $\partial \mathscr{T}(\tau)$. Dizemos que tal superfície é uma superficie marginalmente atrapante.

Dizemos que um espaço $(\mathcal{M}, g)$ é um espaço estacionário regular predizível se satisfaz as condições:

1. $(\mathcal{M}, g)$ é um espaço predizível regular estacionário a partir de uma superfície parcial de Cauchy $\mathscr{S}$

2. Existe um grupo de isometrias $\theta_{t}: \mathcal{M} \rightarrow \mathcal{M}$ cujo vetor de Killing $K$ é temporal perto de $\mathscr{I}^{+}$e $\mathscr{I}^{-}$

3. $(\mathcal{M}, g)$ é vazio ou contém campos que satisfazem a condição dominante de energia: $T_{\alpha \beta} N^{\alpha} L^{\beta} \geqslant 0$ para vetores temporais dirigidos para o futuro $N$ e $L$.

Em um espaço predizível regular estacionário, a área de uma 2-seção do horizonte é independente do tempo.

Proposição 11. Cada componente conexa em $J^{+}\left(\mathscr{I}^{-} \overline{\mathcal{M}}\right)$ do horizonte $\partial \mathscr{B}(\tau)$ em um espaço predizível regular estacionário é homeomorfo a uma 2-esfera.

Definição 1. Uma região atrapante $\mathcal{T}$ do espaço-tempo $\mathcal{M}$ é um subconjunto conexo $\mathcal{T} \subseteq \mathcal{M}$ tal que para todo $p \in \mathcal{T}$, existe uma superfície atrapante $\mathscr{S}$ com $p \in \mathscr{S}$. Uma extensão $\mathcal{T}^{\prime}$ de $\mathcal{T}$ é uma região atrapante com $\mathcal{T} \subset \mathcal{T}^{\prime}$. Uma região atrapante inextendivel é uma região atrapante que não possui extensões. Uma fronteira atrapante é uma componente conexa da fronteira de uma região atrapante inextendivel.

Definição 2. Uma superfície marginal é uma 2-superfície espacial $\mathcal{S}$ em que uma das expansões de geodésicas normais nulas é zero, seja $\left.\theta_{+}\right|_{\mathcal{S}}=0$. Um horizonte atrapante é a clausura $\overline{\mathcal{H}} d e$ uma 3-superfície $\mathcal{H}$ foliada por superfícies marginais nas que $\left.\theta_{-}\right|_{\mathcal{H}} \neq 0$ e $\left.\mathcal{L}_{-} \theta_{+}\right|_{\mathcal{H}} \neq 0$, onde a $\mathcal{L}_{ \pm}$ é a derivada de Lie em relação aos vetores normais à superfície, ver Hayward [1994]. Um horizonte atrapante e uma superfície atrapante são ditas externas se $\left.\mathcal{L}_{-} \theta_{+}\right|_{\mathcal{H}}<0$ ou internas se $\left.\mathcal{L}_{-} \theta_{+}\right|_{\mathcal{H}}>0$, futuras se $\left.\theta_{-}\right|_{\mathcal{H}}<0$ ou passadas se $\left.\theta_{-}\right|_{\mathcal{H}}>0$.

Teorema 1. Dada uma superfície marginal compacta $\mathcal{S}$ morando em um horizonte atrapante, qualquer 2-superfície espacial fechada suficientemente perto de $\mathcal{S}$ é atrapante si esta se encontra dentro do horizonte, ou não atrapante se se encontra fora deste.

Corolário 1. Uma superfície atrapante compacta sempre se encontra dentro da clausura de uma região atrapante. 



\section{ESPAÇO-TEMPOS COM SIMETRIA ESFÉRICA E BURACOS NEGROS}

A métrica mais geral para um sistema esfericamente simétrico pode ser escrita na forma [Cahill and McVittie, 1970]:

$$
d s^{2}=-e^{2 \Pi}\left(d x^{0}\right)^{2}+\left\{e^{2 \Xi}\left(d x^{1}\right)^{2}+Y^{2} d \Omega^{2}\right\}
$$

onde $\Pi, \Xi$ e Y são funções das coordenadas $x^{1}$ e $x^{0}$, enquanto que

$$
d \Omega^{2}=d \vartheta^{2}+\sin ^{2} \vartheta d \varphi^{2}
$$

é o diferencial de ângulo sólido em coordenadas esféricas. Para um sistema esfericamente simétrico a componente $R_{232}^{3}$ do tensor de Riemann é um invariante sobre a transformação de coordenadas $\tilde{x}^{1}=$ $\tilde{x}^{1}\left(x^{1}, x^{0}\right)$ e $\tilde{x}^{0}=\tilde{x}^{0}\left(x^{1}, x^{0}\right)$ [Cahill and McVittie, 1970] (é esfericamente simétrico), e neste sistema de coordenadas pode ser escrita como:

$$
R_{232}^{3}=1+g^{\mu \nu}\left(x^{1}\right)_{, \mu}\left(x^{1}\right)_{, v} .
$$

Agora, no sistema de coordenadas onde $\mathrm{Y}\left(x^{1}, x^{0}\right)=x^{1}=\mathscr{R}$ é a coordenada radial, e $x^{0}$ é a coordenada ortogonal a $\mathscr{R}$ (é a coordenada temporal $t$ ), a métrica (B.1) torna-se:

$$
d s^{2}=-e^{2 \Psi} d t^{2}+\left\{\frac{d \mathscr{R}^{2}}{1-R_{232}^{3}}+\mathscr{R}^{2} d \Omega^{2}\right\},
$$

onde $\Psi$ é uma função de $\mathscr{R}$ e $t$. Se usarmos esta métrica para descrever o interior de uma distribuição esférica de matéria de raio $\mathscr{R}_{\Sigma}$, onde $T^{\mu v}(r)=0$ para $\mathscr{R}>\mathscr{R}_{\Sigma}$; o teorema de unicidade da solução das equações de Einstein para um sistema esfericamente simétrico na ausência de matéria garante que a métrica fora da distribuição de matéria $\left(\mathscr{R}>\mathscr{R}_{\Sigma}\right)$ é dada pela métrica de Schwartzschild:

$$
d s^{2}=-(1-2 M / \mathscr{R}) d t^{2}+\left\{\frac{d \mathscr{R}^{2}}{1-2 M / \mathscr{R}}+\mathscr{R}^{2} d \Omega^{2}\right\}
$$

onde $M$ é uma constante. Se exigirmos adicionalmente que a métrica seja contínua radialmente, em particular na vizinhança da superfície da distribuição de matéria, obtemos que:

$$
\left.R_{232}^{3}\right|_{\Sigma}=\frac{2 M}{\mathscr{R}_{\Sigma}}
$$


Com o que é natural definir a função massa, para a métrica (B.1), como sendo [Cahill and McVittie, 1970]:

$$
m\left(x^{1}, x^{4}\right)=\frac{Y}{2} R_{232}^{3}
$$

Esta função fornece o valor numérico da massa do buraco negro de Schwartzschild avaliada sobre a superfície do núcleo estelar, e vamos usar ela daqui em diante como descrevendo a massa contida pela superfície esférica descrita pelas coordenadas fixas $\left\{x^{1}, x^{4}\right\}$ no interior da distribuição de matéria.

\section{B.1 HORIZONTE APARENTE}

Sabendo que um horizonte aparente (que denotaremos por $h$, ver apêndice A) é sempre um superfície do tipo nula, para um sistema esfericamente simétrico adicionalmente devemos exigir que se ele existe deve então ser uma 2-esfera. Agora, para uma métrica esfericamente simétrica escrita nas coordenadas (B.4) podemos ver que a condição para que o vetor normal a uma superfície esférica de raio $\mathscr{R}$ seja do tipo nulo é [Cai and Wang, 2006]:

$$
\left.g^{\mu v} \mathscr{R}_{, \mu} \mathscr{R}_{, v}\right|_{h}=0, \quad \text { ou }\left.\quad R_{232}^{3}\right|_{h}=1
$$

Usando adicionalmente a definição da massa em volta de uma superfície esfericamente simétrica de raio $\mathscr{R}$ (B.7) obtemos que a massa dentro de um horizonte aparente de raio $\mathscr{R}_{h}$ é:

$$
m_{h}=m\left(\mathscr{R}_{h}, t_{h}\right)=\frac{\mathscr{R}_{h}}{2}
$$

onde $\left\{\mathscr{R}_{h}, t_{h}\right\}$ satisfazem a condição de um horizonte aparente (B.8). Adicionalmente, para sistemas com curvatura espacial constante o escalar de Ricci (2.45) é sempre positivo se o conteúdo material é tal que $\rho_{T}>3 \mathcal{P}_{T}$. Sobre tais condições o horizonte aparente é também um horizonte atrapante [Faraoni, 2011] e sua temperatura associada é sempre positiva [Nielsen, 2009; Di Criscienzo et al., 2010; Vanzo et al., 2011; Iofa, 2015], com o que naturalmente podemos associar a este uma entropia $\mathcal{S}_{h}$ da seguinte forma:

$$
\mathcal{S}_{h}=\frac{k_{B} \mathscr{A}_{h}}{4 \ell_{p l}^{2}} \stackrel{\kappa=0}{\longrightarrow} 4 \pi k_{B}\left(\frac{m_{h}}{m_{p l}}\right)^{2}
$$

onde $k_{B}$ é a constante de Boltzmann, $\mathscr{A}_{h}$ é a área superficial do horizonte aparente e, $\ell_{p l}$ e $m_{p l}$ são o comprimento e a massa de Planck. No caso particular de um universo com um fluido material mais um vácuo dinâmico a primeira derivada da entropia associada ao horizonte aparente $h$ em relação ao fator de escala é

$$
\mathcal{S}_{h}^{\prime}=\frac{9 k_{B}}{8 G \ell_{p l}^{2}} \frac{(1+\omega)}{a} \frac{\rho}{\left(\rho+\rho_{\Lambda}\right)^{2}}>0,
$$

ainda na presença de curvatura. 


\section{B.2 CONDIÇÕES DE JUNÇÃO}

No que segue vamos considerar um sistema esfericamente simétrico sobre o processo de colapso gravitacional. Este sistema pode ser naturalmente dividido em duas variedades separadas pela hiper-superfície que define a fronteira do corpo colapsante $\Sigma$, descritos pelas coordenadas $x_{-}^{\alpha}$ e $x_{+}^{\alpha}$ para o interior e o exterior, respectivamente, e com métricas:

$$
d s_{\Sigma}^{2}=g_{a b} d \xi^{a} d \xi^{b}
$$

para a hiper-superfície $\Sigma$, e

$$
d s_{ \pm}^{2}=g_{\alpha \beta} d x_{ \pm}^{\alpha} d x_{ \pm}^{\beta}
$$

pára as variedades externa e interna, respectivamente. Seguindo o procedimento de [Santos, 1985] definimos as condições de junção entre as variedades interna e externa pela igualdade da primeira e segunda formas fundamentais das duas variedades sobre a hiper-superfície $\Sigma$ :

$$
\left(d s_{+}^{2}\right)_{\Sigma}=\left(d s_{-}^{2}\right)_{\Sigma}=d s_{\Sigma}^{2},\left.\quad \quad K_{\mu v}^{-}\right|_{\Sigma}=\left.K_{\mu v}^{+}\right|_{\Sigma},
$$

onde a segunda forma fundamental [Santos, 1985] pode ser expressa como:

$$
K_{a b}=-n_{\lambda} \frac{\partial x^{\lambda}}{\partial \xi^{a} \partial \xi^{b}}-n_{\lambda} \Gamma_{\mu v}^{\lambda} \frac{\partial x^{\mu}}{\partial \xi^{a}} \frac{\partial x^{v}}{\partial \xi^{b}}
$$

para implementar as entre os espaço-tempos interno $(-)$ e externo $(+)$ na sua fronteira $(\Sigma)$, e onde os índices latino podem ser 0,2,3, enquanto que os índices gregos barrem 0,1,2,3 em nosso sistema esfericamente simétrico.

A métrica intrínseca na hiper-superfície $\Sigma$ vai ser escrita como:

$$
d s_{\Sigma}^{2}=g_{a b} d \xi^{a} d \xi^{b}=-d \tau^{2}+R^{2}(\tau) d \Omega^{2}
$$

onde as coordenadas $\xi^{a}$ são $\tau, \theta$ e $\phi$, enquanto que $d \Omega^{2}=d \theta^{2}+\sin ^{2} \theta d \phi^{2}$.

\section{B.3 MÉTRICA INTERNA}

Usando a métrica interna proposta por [Santos, 1985], para um observador comóvel

$$
d s^{2}=-d t^{2}+B^{2}(r, t)\left(d r^{2}+r^{2} d \Omega^{2}\right)
$$

e definindo a fronteira $\Sigma$ como: $f(r, t)=r-r_{\Sigma}=0$, donde $r_{\Sigma}$ é uma constante, segue que o vetor perpendicular à superfície $f(r, t)$ esta dado por:

$$
\frac{\partial f}{\partial x_{-}^{\alpha}}=\left\{\frac{\partial f}{\partial t}, \frac{\partial f}{\partial r}, \frac{\partial f}{\partial \theta}, \frac{\partial f}{\partial \phi}\right\}_{\Sigma}=\{0,1,0,0,0\},
$$


e sua normalização é:

$$
n_{\alpha}^{-}=\frac{\partial f}{\partial x_{-}^{\alpha}}\left(g^{\mu v} \frac{\partial f}{\partial x_{-}^{\mu}} \frac{\partial f}{\partial x_{-}^{v}}\right)^{-1 / 2}=\{0, B(r, t), 0,0\}
$$

Por outro lado, os símbolos de Christofel para a métrica (B.17) estão dados por [Cahill and McVittie, 1970]:

$$
\begin{array}{llll}
\Gamma_{01}^{1}=\frac{\partial \ln B}{\partial t}, & \Gamma_{00}^{0}=\frac{\partial \ln B}{\partial t}, & \Gamma_{12}^{2}=\frac{\partial \ln (B r)}{\partial r}, & \Gamma_{13}^{3}=\frac{\partial \ln (B r)}{\partial r}, \\
\Gamma_{11}^{1}=\frac{\partial \ln B}{\partial r}, & \Gamma_{11}^{0}=B \frac{\partial B}{\partial t}, & \Gamma_{24}^{2}=\frac{\partial \ln (B r)}{\partial t}, & \Gamma_{23}^{3}=\cot \theta, \\
\Gamma_{22}^{1}=-\frac{r}{B} \frac{\partial(B r)}{\partial r}, & \Gamma_{22}^{0}=B r \frac{\partial(B r)}{\partial t}, & \Gamma_{33}^{2}=-\sin \theta \cos \theta, & \Gamma_{34}^{3}=\frac{\partial \ln (B r)}{\partial t}, \\
\Gamma_{33}^{1}=\sin ^{2} \theta \Gamma_{22}^{1}, & \Gamma_{33}^{4}=\sin ^{2} \theta \Gamma_{22}^{0}, & &
\end{array}
$$

com isto, as componentes da segunda forma fundamental sobre a hiper-superfície $\Sigma$ são:

$$
K_{\tau \tau}^{-}=0, \quad K_{\theta \theta}^{-}=\frac{K_{\phi \phi}^{-}}{\sin ^{2} \theta}=\left(r \frac{\partial(B r)}{\partial r}\right)_{\Sigma}
$$

\section{B.3.1 Equações de Einstein}

Para o tensor momento energia em coordenadas comóveis:

$$
T_{\mu v}=(\rho+\mathcal{P}) U_{\mu} U_{v}+\mathcal{P} g_{\mu v}+q_{\mu} U_{v}+q_{\nu} U_{\mu}
$$

onde $U^{\alpha}=\delta_{0}^{\alpha}, \rho$ e $\mathcal{P}$ são a quadrivelocidade, densidade de energia e a pressão do fluido, $q^{\alpha}=q \delta_{1}^{\alpha}$ é o fluxo radial de calor (dado que estamos assumindo simetria esférica), as equações de Einstein são [Santos, 1985]:

$$
\begin{gathered}
G_{00}=\frac{-1}{B^{2}}\left\{\frac{2}{B} \frac{\partial^{2} B}{\partial r^{2}}-\frac{1}{B^{2}}\left(\frac{\partial B}{\partial r}\right)^{2}+\frac{4}{r B} \frac{\partial B}{\partial r}\right\}+\frac{3}{B^{2}}\left(\frac{\partial B}{\partial t}\right)^{2}=8 \pi G \rho, \\
G_{11}=\frac{1}{B^{2}}\left(\frac{\partial B}{\partial r}\right)^{2}+\frac{2}{r B} \frac{\partial B}{\partial r}-B^{2}\left\{\frac{2}{B} \frac{\partial^{2} B}{\partial t^{2}}+\frac{1}{B^{2}}\left(\frac{\partial B}{\partial t}\right)^{2}\right\}=8 \pi G \mathcal{P} B^{2}, \\
G_{22}=r^{2}\left\{\frac{1}{B} \frac{\partial^{2} B}{\partial r^{2}}-\frac{1}{B^{2}}\left(\frac{\partial B}{\partial r}\right)^{2}+\frac{1}{r B} \frac{\partial B}{\partial r}\right\}-r^{2} B^{2}\left\{\frac{2}{B} \frac{\partial^{2} B}{\partial t^{2}}+\frac{1}{B^{2}}\left(\frac{\partial B}{\partial t}\right)^{2}\right\}=8 \pi G \mathcal{P} B^{2} r^{2}, \\
G_{01}=-\frac{2}{B} \frac{\partial^{2} B}{\partial r \partial t}+\frac{2}{B^{2}} \frac{\partial B}{\partial r} \frac{\partial B}{\partial t}=-8 \pi G q B^{2},
\end{gathered}
$$


o calculo de $\left(G_{11}-r^{-2} G_{22}\right)$ produz a relação

$$
\frac{\partial}{\partial r}\left(\frac{1}{r B^{2}} \frac{\partial B}{\partial r}\right)=0
$$

que tem como solução

$$
B(r, t)=\frac{f(t)}{g(t) r^{2}+1}, \quad \rightarrow \quad\left(\frac{1}{B} \frac{\partial B}{\partial t}\right)=\left(\frac{1}{f} \frac{\partial f}{\partial t}\right)-\frac{g r^{2}}{g r^{2}+1}\left(\frac{1}{g} \frac{\partial g}{\partial t}\right)
$$

a equação (B.23d) pode se reescrever como:

$$
\frac{\partial}{\partial r}\left(\frac{1}{B} \frac{\partial B}{\partial t}\right)=4 \pi G q B^{2}
$$

e, usando a solução para B (B.25), ainda pode se escrever como:

$$
q(r, t)=-\frac{r}{2 \pi G} \frac{1}{f^{2}} \frac{\partial g}{\partial t}
$$

Da relação $\left(B^{2} G_{00}+G_{11}+2 r^{-2} G_{22}\right)$ se obtém

$$
8 \pi G(\rho+3 \mathcal{P})+\frac{6}{B} \frac{\partial^{2} B}{\partial t^{2}}=0,
$$

enquando que da relação $\left(B^{2} G_{00}+G_{11}\right)$ obtemos

$$
8 \pi G(\rho+\mathcal{P})+2 \frac{\partial}{\partial t}\left(\frac{1}{B} \frac{\partial B}{\partial t}\right)+\frac{8 g}{f^{2}}=0,
$$

e juntando-lha com a equação anterior $\left(G_{00}+G_{11}-r^{-2} G_{22}\right)$ obtemos que

$$
8 \pi G \rho=3\left(\frac{1}{B} \frac{\partial B}{\partial t}\right)^{2}-12 \frac{g}{f^{2}}
$$

por outro lado, das equações de conservação do tensor momento energia obtemos [Cahill and McVittie, 1970]:

$$
\begin{gathered}
\frac{\partial \rho}{\partial t}+3\left(\frac{1}{B} \frac{\partial B}{\partial t}\right)(\rho+\mathcal{P}+q)+3 \frac{q}{r}=0 \\
\frac{\partial q}{\partial t}+5\left(\frac{1}{B} \frac{\partial B}{\partial t}\right) q+\frac{1}{B^{2}} \frac{\partial \mathcal{P}}{\partial r}=0
\end{gathered}
$$




\section{B.4 MÉTRICA EXTERNA}

Partindo da métrica externa escrita em coordenadas esféricas como

$$
d s^{2}=-\left(1-\frac{2 m}{r}-\frac{\Lambda r^{2}}{3}\right) d t^{2}+\left(1-\frac{2 m}{r}-\frac{\Lambda r^{2}}{3}\right)^{-1} d r^{2}+r^{2} d \Omega^{2}
$$

podemos fazer uma primeira mudança de variável:

$$
\begin{gathered}
d r^{*}=\left(1-\frac{2 m}{r}-\frac{\Lambda r^{2}}{3}\right)^{-1} d r \\
d s^{2}=-\left(1-\frac{2 m}{r}-\frac{\Lambda r^{2}}{3}\right)\left(d t^{2}-d r^{* 2}\right)+r^{2} d \Omega^{2}
\end{gathered}
$$

se agora fazemos mudança

$$
\begin{gathered}
d v=d t-d \mathrm{r}^{*}, \quad d u=d t+d \mathrm{r}^{*}, \\
d s^{2}=-\left(1-\frac{2 m}{r}-\frac{\Lambda r^{2}}{3}\right) d u d v+r^{2} d \Omega^{2},
\end{gathered}
$$

e usamos $d u=d v+2 d$ r $^{*}$ podemos reescrever a métrica como sendo

$$
d s^{2}=-\left(1-\frac{2 m}{r}-\frac{\Lambda r^{2}}{3}\right) d v^{2}-2 d v d r+r^{2} d \Omega^{2}
$$

Neste sistema de coordenadas os símbolos de Christoffel são:

$$
\begin{array}{llll}
\Gamma_{00}^{0}=-\frac{m}{r^{2}}+\frac{\Lambda r}{3}, & \Gamma_{22}^{0}=r, & \Gamma_{33}^{0}=r \sin ^{2} \theta, & \\
\Gamma_{00}^{1}=-\frac{1}{r} \frac{\partial m}{\partial v}+\frac{\Gamma_{00}^{0} \Gamma_{22}^{1}}{r}, & \Gamma_{10}^{1}=\frac{m}{r^{2}}-\frac{\Lambda r}{3}, & \Gamma_{22}^{1}=-r+2 m+\frac{\Lambda r^{3}}{3}, & \Gamma_{33}^{1}=\sin ^{2} \theta \Gamma_{22}^{1}, \\
\Gamma_{21}^{2}=\frac{1}{r^{\prime}} & \Gamma_{33}^{2}=-\sin \theta \cos \theta, & \Gamma_{31}^{3}=\frac{1}{r}, & \Gamma_{32}^{3}=\cot \theta,
\end{array}
$$

como que as componentes não triviais do tensor de Einstein podem escreve-se como

$$
\begin{array}{ll}
G_{00}=\Lambda-\frac{r^{2} \Lambda^{2}}{3}-\frac{2 \Lambda m}{r}-\frac{2}{r^{2}} \frac{\partial m}{\partial u}, & G_{01}=\Lambda, \\
G_{22}=r^{2} \Lambda, & G_{33}=-r^{2} \Lambda \sin ^{2} \theta .
\end{array}
$$

Se definirmos a fronteira $\Sigma$ como sendo a hiper-superfície tal que $f(r, v)=\varangle-r_{\Sigma}(v)=0$, teremos que

$$
\frac{\partial f}{\partial x_{+}^{\alpha}}=\left\{-\frac{d r_{\Sigma}}{d v}, 1,0,0\right\}_{\Sigma}
$$


é um vetor perpendicular à superfície $\Sigma$, e a partir dele podemos escrever o vetor normal a $\Sigma$ como sendo

$$
n_{\alpha}^{+}=\left.\left(2 \frac{d r_{\Sigma}}{d v}+1-\frac{2 m}{r}-\frac{\Lambda r^{2}}{3}\right)^{-1 / 2}\left\{-\frac{d r_{\Sigma}}{d v}, 1,0,0\right\}\right|_{\Sigma} .
$$

Se agora usarmos as condições de junção para a primeira forma fundamental de $\Sigma$ encontra-se que

$$
\left.\left(\frac{d v}{d \tau}\right)^{2}\left(1-\frac{2 m}{\mathbb{r}}-\frac{\Lambda r^{2}}{3}+2 \frac{d r}{d v}\right)\right|_{\Sigma}=1
$$

com o que podemos reescrever o vetor normal à superfície como

$$
n_{\alpha}^{+}=\left\{-\frac{d{ }_{\Sigma}}{d \tau}, \frac{d v}{d \tau}, 0,0\right\}
$$

com o que agora podemos calcular a segunda forma diferencial como sendo:

$$
\begin{aligned}
& K_{\tau \tau}^{+}=\frac{d^{2} v / d \tau^{2}}{d v / d \tau}-\left.\frac{d v}{d \tau}\left(\frac{m}{r}-\frac{\Lambda r}{3}\right)\right|_{\Sigma}, \\
& K_{\theta \theta}^{+}=K_{\phi \phi}^{+} \sin ^{-2} \theta=r \frac{d r}{d \tau}+\left.r \frac{d v}{d \tau}\left(1-\frac{2 m}{r}-\frac{\Lambda r^{2}}{3}\right)\right|_{\Sigma} .
\end{aligned}
$$

Impondo a condição de junção sobre as componentes da segunda forma diferencial, podemos escrever a massa vista por um observador externo (B.33) como função das coordenadas internas (B.17) da seguinte forma:

$$
m=-\frac{\Lambda B^{3} r^{3}}{6}+\frac{B r^{3}}{2}\left(\frac{\partial B}{\partial t}\right)^{2}-\frac{r^{3}}{2 B}\left(\frac{\partial B}{\partial r}\right)^{2}-\left.r^{2} \frac{\partial B}{\partial r}\right|_{\Sigma}
$$

é equivalente à massa definida por Cahill e McVittie [Cahill and McVittie, 1970] da equação (B.7) no caso em que $\Lambda=0$. Adicionalmente, as condições de junção, e as equações de Einstein do espaço-tempo interno, produzem a seguinte relação, que deve ser respeitada sobre a hiper-superfície $\Sigma$ :

$$
\mathcal{P}_{\Sigma}=\left.q B\right|_{\Sigma}+\frac{\Lambda}{24 \pi G}
$$

o que obriga à pressão total interna avaliada sobre a superfície do corpo colapsante a se anular no caso em que tanto o fluxo de calor, sobre a superfície, como a constante $\Lambda$ sejam nulos. 



\section{BIBLIO GR A F I A}

M. Abramowitz and I. A. Stegun. Handbook of Mathematical Functions. Dover Publications, New York, 1964.

S. L. Adler. Rev. Mod. Phys., 54(3):729-766, 1982.

J. S. Alcaniz and J. A. S. Lima. Phys. Rev. D, 72(063516):1-7, 2005.

L. Anderson et al. MNRAS, 427:3435-3467, 2012.

A. I. Arbab. Gen. Rel. Grav., 29(1):61-74, 1997.

A. I. Arbab and A. M. M. Abdel-Rahman. Phys. Rev. D, 50(12):7725-7728, 1994.

R. C. Arcuri and I. Waga. Phys. Rev. D, 50:2928-2931, 1994.

M. J. D. Assad and J. A. S. Lima. Gen. Rel. Grav., 20(6):527-538, 1988.

A. Babić, B. Guberina, R. Horvat, and H. Štefančić. Phys. Rev. D, 65(085002):1-6, 2002.

A. Babić, B. Guberina, R. Horvat, and H. Štefančić. Phys. Rev. D, 71(124041):1-10, 2005.

J. M. Bardeen. Phys. Rev. D, 22(8):1882-1905, 1980.

S. Basilakos, M. Plionis, and J. Solà. Phys. Rev. D, 80(083511):1-19, 2009.

S. Basilakos, M. Plionis, and J. Solà. Phys. Rev. D, 82(083512):1-14, 2010.

S. Basilakos, D. Polarski, and J. Solà. Phys. Rev. D, 86(043010):1-13, 2012.

J. Beringer et al. Phys. Rev. D, 86(010001):1-1526, 2012.

F. Beutler et al. MNRAS, 423(3430), 2012.

C. Blake. MNRAS, 415:2892-2909, 2011.

L. F. Bloomfield Torres and I. Waga. MNRAS, 279:712-726, 1996.

I. L. Buchbinder, S. D. Odintsov, and I. L. Shapiro. Effective action in quantum gravity. IOP Publishing Ltd, London, 1992.

M. E. Cahill and G. C. McVittie. J. Math. Phys., 11(4):1382-1391, 1970.

R-G. Cai and A. Wang. Phys. Rev. D, 73(063005):1-12, 2006.

M. Campos and J. A. S. Lima. Phys. Rev. D, 86(043012):1-9, 2012.

S. Carneiro. Int. J. Mod. Phys. D, 15(12):2241-2247, 2006.

S. Carneiro and R. Tavakol. Int. J. Mod. Phys. D, 18(14):2343-2349, 2009. 
S. Carroll. Spacetime and Geometry. Wesley, Addison, 2004.

J. C. Carvalho, J. A. S. Lima, and I. Waga. Phys. Rev. D, 46:2404-2407, 1992.

W. Chen and Y-S. Wu. Phys. Rev. D, 41(2):695-698, 1990.

E. J. Copeland, M. Sami, and S. Tsujikawa. Int. J. Mod. Phys. D, 15(11):1753-1935, 2006.

F. E. M. Costa, J. S. Alcaniz, and J. M. F. Maia. Phys. Rev. D, 77(083516):1-6, 2008.

F. E. M. Costa, J. A. S. Lima, and F. A. Oliveira. arXiv:1204.1864, 2012.

R. Di Criscienzo, S. A. Hayward, M. Nadalini, L. Vanzo, and S. Zerbini. Classical and Quantum Gravity, 27(1):015006, 2010.

S. Dodelson. Modern Cosmology. Academic Press, 2003.

L. P. Eisenhart. Riemanninan Geometry. Geoffrey Cumberlege, Oxford Univ. Press, 1949.

D. J. Eisenstein and W. Hu. Astrophys. J., 496(2):605-614, 1998.

C. España-Bonet, P. Ruiz-Lapuente, I. L. Shapiro, and J. Solà. JCAP, 02(006), 2004.

J. C. Fabris, I. L. Shapiro, and J. Solà. JCAP, 02(016), 2007.

V Faraoni. Phys. Rev. D, 84(024003):1-15, 2011.

O. Farooq and B. Ratra. Astrophys. J., 766(L7), 2013.

P. G. Ferreira and M. Joyce. Phys. Rev. D, 58(023503):1-23, 1998.

K. Freese et al. Nucl. Phys. B, 287:797-814, 1987.

J. A. Frieman, M. S. Turner, and D. Huterer. Ann. Rev. Astro. Astrophys., 46:385-432, 2008.

J. Grande, J. Solà, S. Basilakos, and M. Plionis. JCAP, 08(007), 2011.

R. Griostri et al. JCAP, 03(027), 2012.

S. W. Hawking and G. F. R. Ellis. The Large Scaçe Structure of Spacetime. Cambridge University Press, Cambridge, 1973.

S. A. Hayward. Phys. Rev. D, 49(12):6467-6474, 1994.

S. A. Hayward. Phys. Rev. D, 70(104027):1-13, 2004.

M. Hicken et al. Astrophys. J., 700:1097-1140, 2009.

G. Hinshaw et al. Astrophys. J. Suppl. Ser., 180:225-245, 2009.

M. Z. Iofa. Phys. Rev. D, 91:024046, 2015.

J. F. Jesus, R. C. Santos, J. S. Alcaniz, and J. A. S. Lima. Phys. Rev. D, 78(063514):1-7, 2008.

E. W. Kolb and M. S. Turner. The early universe. Addison-Wesley, 1988.

E. Komatsu et al. Astrophys. J. Suppl. Ser., 180:330-370, 2009. 
E. Komatsu et al. Astrophys. J. Suppl. Ser., 192(18):1-47, 2011.

M. Kowalski et al. Astrophys. J., 686:749-778, 2008.

L. M. Krauss. Astron. J., 501:461-466, 1998.

J. A. S. Lima. Phys. Rev. D, 54(4):2571-2577, 1996.

J. A. S. Lima. Gen. Rel. Grav., 29(7):805-813, 1997.

J. A. S. Lima. Am. J. Phys., 69(1245):1245-1247, 2001.

J. A. S. Lima and A. Maia. Phys. Rev. D, 52(10):5628-5635, 1995.

J. A. S. Lima and J. M. F. Maia. Phys. Rev. D, 49(10):5597-5600, 1994.

J. A. S. Lima and S. H. Pereira. Phys. Rev. D, 78(083504):1-7, 2008.

J. A. S. Lima and J. Santos. Int. J. Theor. Phys., 34(1):127-134, 1995.

J. A. S. Lima and M. Trodden. Phys. Rev. D, 53(8):4280-4286, 1996.

J. A. S. Lima, S. Basilakos, and J. Solà. MNRAS, 431:923-929, 2013.

J. A. S. Lima, M. Campos, and E. L. D. Perico. em preparação, 2015a.

J. A. S. Lima, E. L. D. Perico, and G. J. M. Zilioti. IJMPD, 24(4):1541006, 2015b.

C-P Ma and E. Bertschinger. Astrophys. J., 455:7-25, 1995.

G. C. McVittie. MNRAS, 93(5):325-339, 1933.

N. Metropolis, A. W. Rosenbluth, M. N. Rosenbluth, A. H. Teller, and E. Teller. J. Chem. Phys., 21 (1087), 1953.

J. P. Mimoso and D. Pavón. Phys. Rev. D, 87(047302):1-5, 2013.

V. F. Mukhanov. Physical Foundations of Cosmology. Cambridge Un. Press, 2005.

V. F. Mukhanov, H. A. Feldman, and F. H. Brandenberger. Phys. Rep., 215(5 \& 6):203-333, 1992.

J. V. Narlikar. An introduction to cosmology. Cambridge Un. Press, 2002.

B. L. Nelson and P. Panangaden. Phys. Rev. D, 25(4):1019-1027, 1982.

A. B. Nielsen. General Relativity and Gravitation, 41(7):1539-1584, 2009. ISSN 0001-7701.

R. Opher and A. Pelinson. Phys. Rev. D, 70(063529):1-4, 2004.

R. Opher and A. Pelinson. MNRAS, 362:167-170, 2005.

J. M. Overduin and S. Cooperstock. Phys. Rev. D, 58(043506):1-23, 1998.

M. Ozer and O. Taha. Nucl. Phys. B, 287:776-796, 1987.

T. Padmanabhan. Phys. Rep., 380:235-320, 2003. 
L. Parker and D. J. Toms. Phys. Rev. D, 32(6):1409-1420, 1985.

D. Pavón. Phys. Rev. D, 43(2):375-378, 1991.

J. A. Peacock. Cosmological physics. Cambridge Un. Press, 1999.

P. J. E. Peebles. The large escale of the universe. Princeton Un. Press, 1980.

P. J. E. Peebles and B. Ratra. Rev. Mod. Phys., 75:559-606, 2003.

R. Penrose. Gen. Rel. Grav., 34(7):1141-1165, 2002.

W. J. Percival. Astrophys. J., 657:645-663, 2007. doi: 10.1086/510615.

W. J. Percival et al. MNRAS, 353(1201), 2004.

W. J. Percival et al. MNRAS, 401:2148-2168, 2010.

S. H. Pereira and J. A. S. Lima. Phys. Lett. B, 669:266-270, 2008.

E. L. D. Perico and J. A. S. Lima. em preparação, 2015.

E. L. D. Perico, J. A. S. Lima, S. Basilakos, and J. Solà. Phys. Rev. D, 88(063531):1-14, 2013a.

E. L. D. Perico, J. A. S. Lima, and M. Campos. arXiv:1303.0430, 2013b.

S. Perlmutter et al. Nature, 391:51-54, 1998.

S. Perlmutter et al. Astrophys. J., 517:565-586, 1999. doi: 10.1086/307221.

Planck Collaboration. $A \& \&$ A, 2014.

E. Poisson and W. Israel. Phys. Rev. D, 41(6):1796-1809, 1990.

R. O. Ramos, M. V. Santos, and I. Waga. Phys. Rev. D, 89(083524):1-10, 2014.

B. Ratra and P. J. E. Peebles. Phys. Rev. D, 37(12):3406-3427, 1988.

B. A. Ried et al. MNRAS, 426(2719), 2012.

A. G. Riess et al. Astron. J., 116:1009-1038, 1998. doi: 10.1086/300499.

J. Salim and I. Waga. Class. Quant. Grav., 10:1767-1774, 1993.

L. Samushina, W. J. Percival, and A. Raccanelli. MNRAS, 420(2102), 2012.

N. O. Santos. MNRAS, 216:403-410, 1985.

I. L. Shapiro and J. Solà. JHEP, 02(006), 2002.

I. L. Shapiro and J. Solà. Phys. Lett. B, 682:105-113, 2009.

I. L. Shapiro, J. Solà, C. España-Bonet, and P. Ruiz-Lapuente. Phys. Lett. B, 574:149-155, 2003.

I. L. Shapiro, J. Solà, and H. Štefančić. JCAP, 01(012), 2005.

R. Silva, J. A. S. Lima, and M. O. Calvão. Gen. Rel. Grav., 34(6):865-875, 2002. 
R. Silva, J. S. Alcaniz, and J. A. S. Lima. Int. J. Mod. Phys. D, 16(2\&3):469-473, 2007.

J. Solà. J. Phys. A: Math. Theor., 41(164066):1-12, 2008.

J. Solà. J. Phys. Conf. Ser., 283(012033):1-14, 2011.

J. Solà and H. Štefančić. Phys. Lett. B, 624:147-157, 2005.

J. Solà and H. Štefančić. Mod. Phys. Lett. A, 21(6):479-494, 2006.

J. Sollerman et al. Astron. J., 703(2):1374-1385, 2009.

D. N. Spergel et al. Astrophys. J. Suppl. Ser., 148:175-194, 2003.

D. N. Spergel et al. Astrophys. J. Suppl. Ser., 170:377-408, 2007.

N. Suzuki et al. Astrophys. J., 746(853):1-24, 2012.

D. J. Toms. Phys. Lett. B, 126(2):37-40, 1983.

S. Torre et al. $A \& \&$ A, 557(A54), 2013.

P. C. Vaidya. Proc. Indian Acad. Sci. A, 33:264-276, 1951.

P. C. Vaidya. Nature, 171(4345):260-261, 1953.

P. C. Vaidya. Astrophys. J., 144:943-952, 1966.

L. Vanzo, G. Acquaviva, and R. Di Criscenzo. arXiv:1106.4153, 2011.

J. F. Villas da Rocha, A. Wang, and N. O. Santos. Phys. Lett. A, 255:213-220, 1999.

I. Waga. Astrophys. J., 414:436-448, 1993.

A. Wang, J. F. Villas da Rocha, and N. O. Santos. Phys. Rev. D, 56(12):7692-7699, 1997.

P. Wang and X-M Meng. Class. Quant. Grav., 22:283-294, 2005.

S. Weinberg. Gravitation and cosmology. Jonh Wiley \& Sons, 1972.

S. Weinberg. Rev. Mod. Phys., 61(1):1-23, 1989.

C. Wetterich. $A \mathscr{E}$ A, 301:321-328, 1995.

WMAP. URL http://lambda.gsfc.nasa.gov/product/map/dr5/parameters.cfm.

L. Xu, Y. Wang, M. Tong, and H. Noh. Phys. Rev. D, 84(123004):1-8, 2011.

Ya. B. Zel'dovich. Sov. Phys. Usp., 11(3):381-393, 1968. 